\title{
Improving Therapies of Rhabdomyosarcoma
}

\section{Dissertation}

\author{
for the award of the degree \\ "Doctor rerum naturalium (Dr. rer. nat.)" \\ of the Georg-August University Göttingen
}

within the doctoral program Molecular Medicine

of the Georg-August University School of Science (GAUSS)

submitted by

Rosalie Ridzewski

from Erfurt

Göttingen 2015 


\section{Members of the Thesis Committee}

Supervisor:

\section{Prof. Dr. Heidi Hahn}

Dept. of Human Genetics; University Medical Center Göttingen

Second member of the thesis committee:

\section{Prof. Dr. Matthias Dobbelstein}

Dept. of Molecular Oncology; Georg-August-University Göttingen

Third member of the thesis committee:

Prof. Dr. Dieter Kube

Dept. of Haematology and Oncology; University Medical Center Göttingen

Date of Disputation: 07.12.2015 


\section{Affidavit}

Here I declare that my doctoral thesis entitled "Improving Therapies of Rhabdomyosarcoma" has been written independently with no other sources and aids than quoted.

Rosalie Ridzewski

Göttingen, October 2015 
Parts of this work have been published:

Ridzewski, R., Rettberg, D., Dittmann, K., Cuvelier, N., Fulda, S., Hahn, H., 2015. Hedgehog Inhibitors in Rhabdomyosarcoma: A Comparison of Four Compounds and Responsiveness of Four Cell Lines. Frontiers in oncology 5, 130 


\section{Contents}

AFFIDAVIT ......................................................................................................................... III

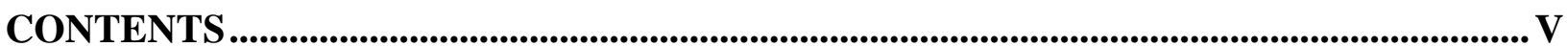

LIST OF FIGURES ............................................................................................................ IX

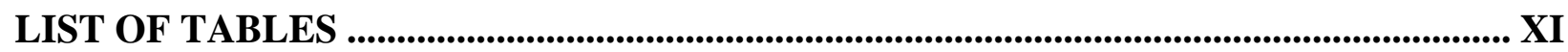

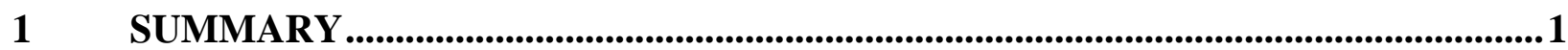

2 INTRODUCTION .............................................................................................................

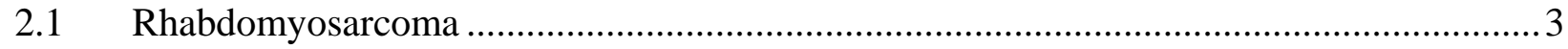

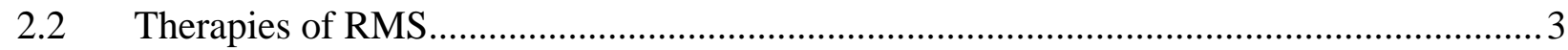

2.3 The hedgehog signaling pathway ..........................................................................

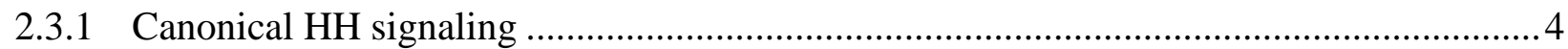

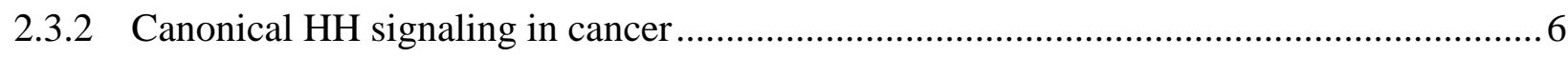

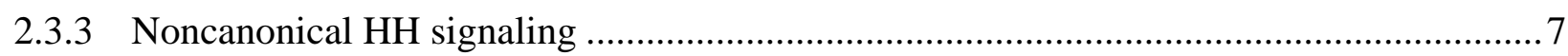

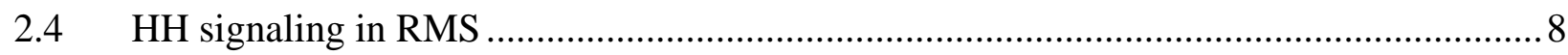

2.5 HH inhibitors - SMO antagonists ..........................................................................

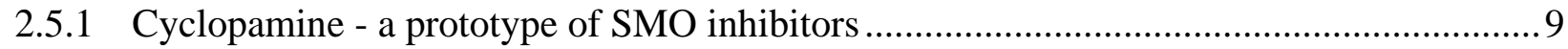

2.5.2 GDC-0449, LDE225 and HhA - more potent derivatives .............................................. 10

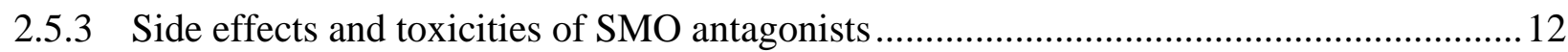

2.5.4 Mechanisms of acquired resistance to SMO inhibitors ................................................. 12

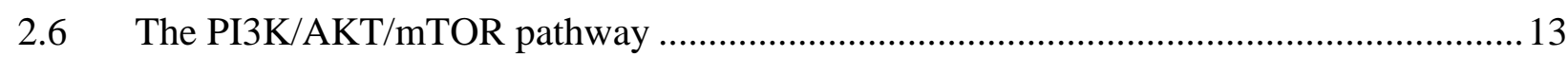

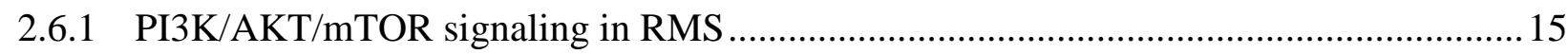

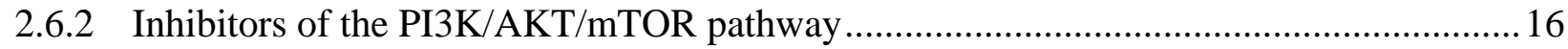

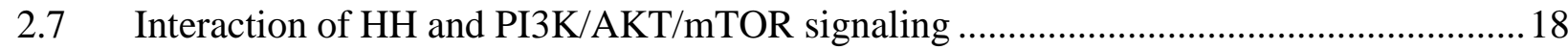

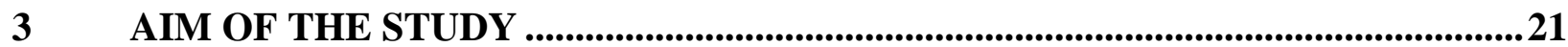

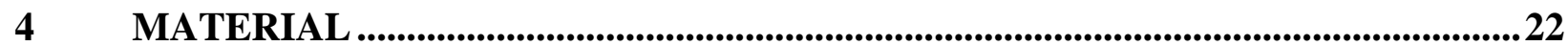

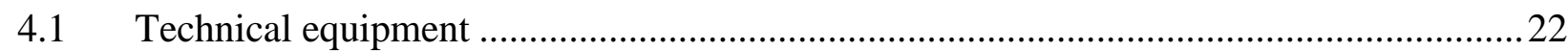

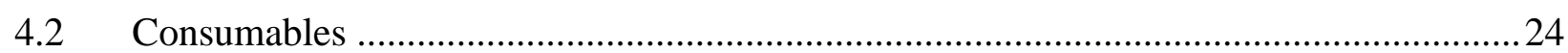

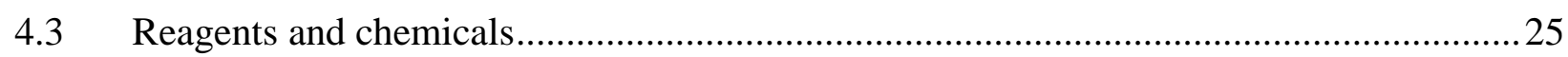

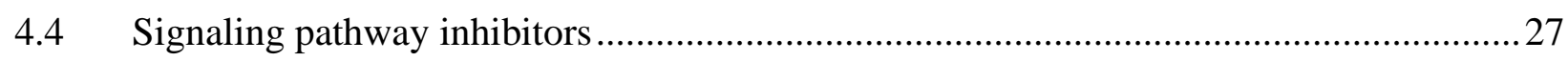

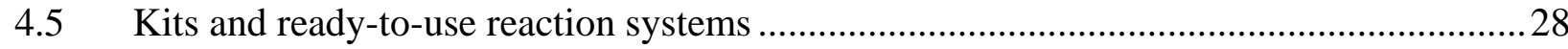

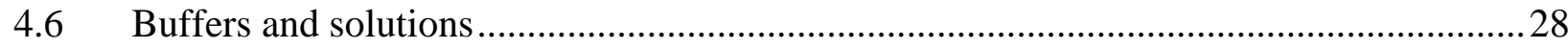




\section{CONTENTS}

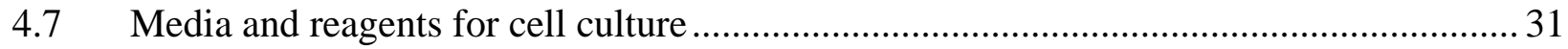

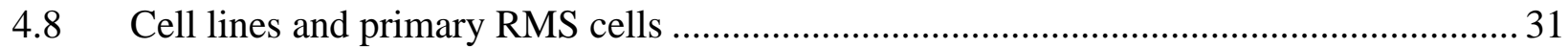

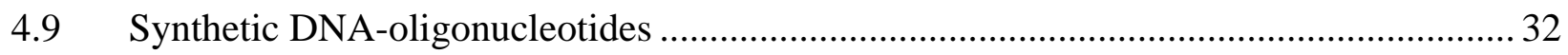

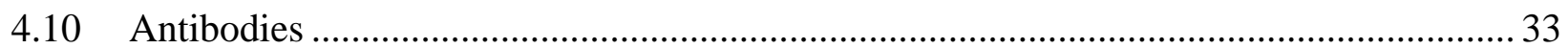

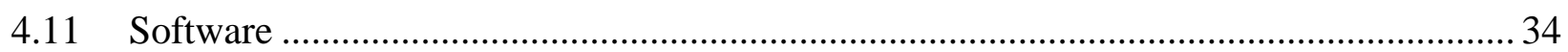

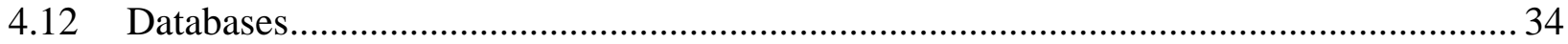

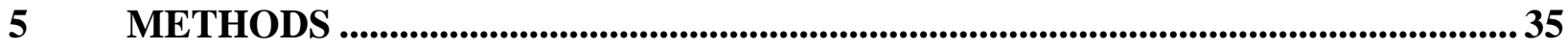

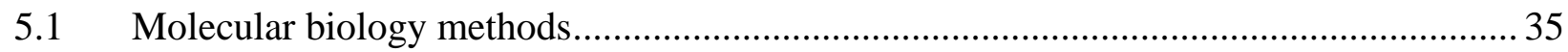

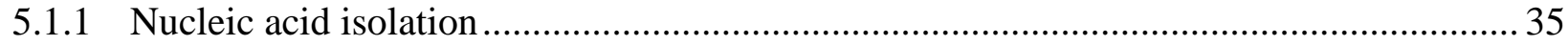

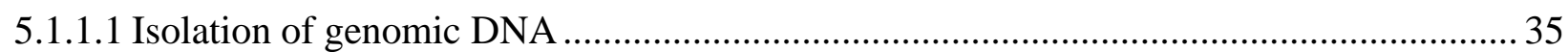

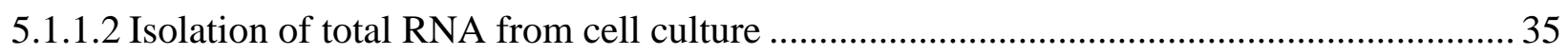

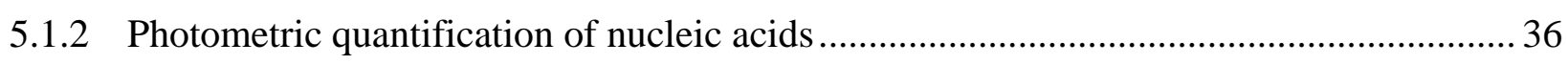

5.1.3 Reverse transcription of RNA (cDNA synthesis) .................................................... 36

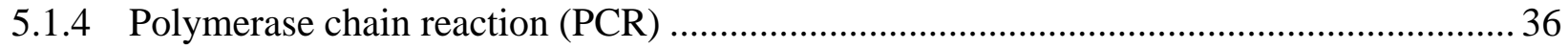

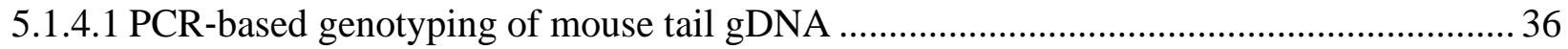

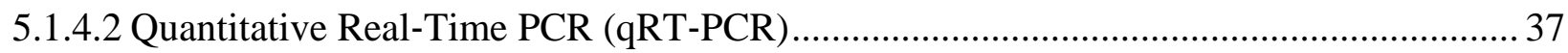

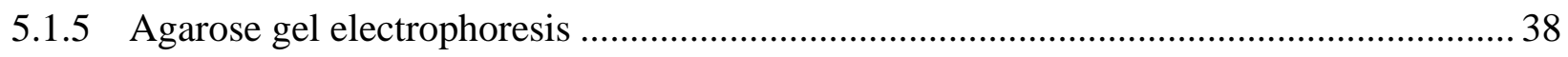

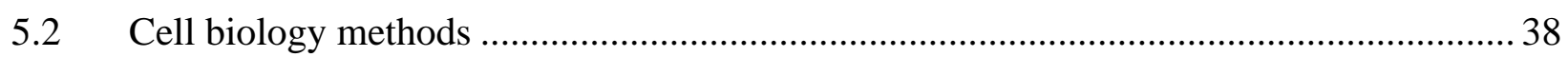

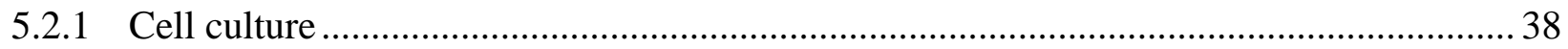

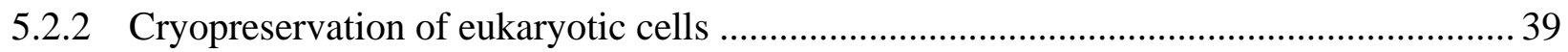

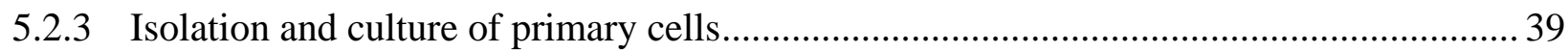

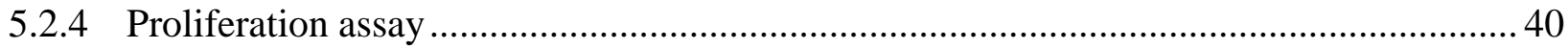

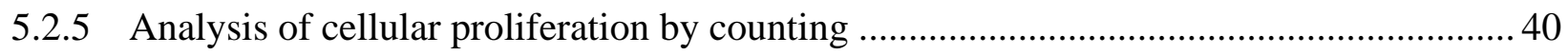

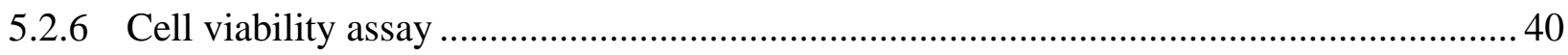

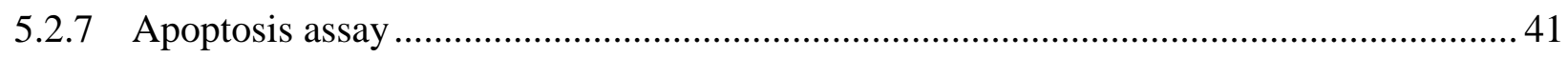

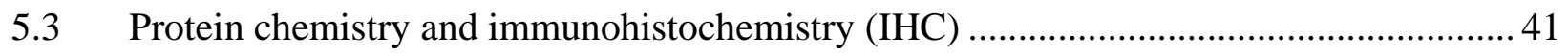

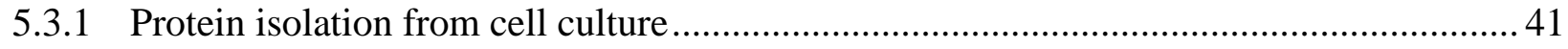

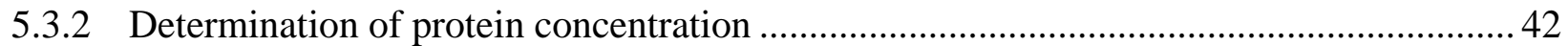

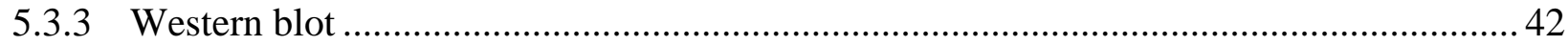

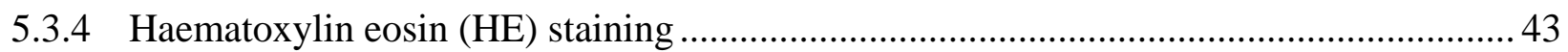

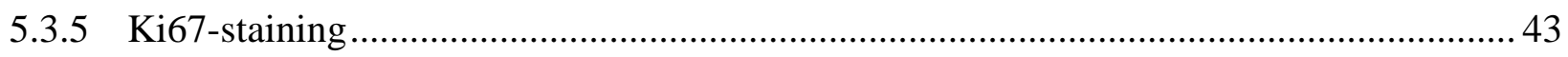

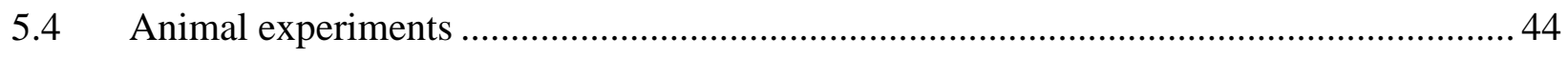

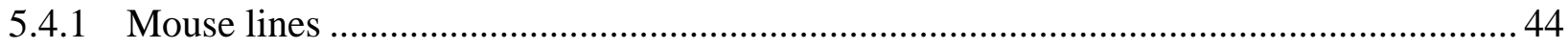

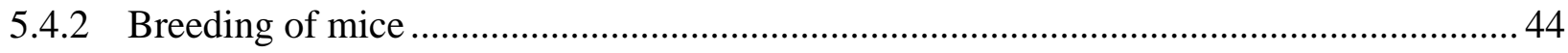


5.4.3 Tail biopsy and genotyping of mice .44

5.4.4 Monitoring of RMS bearing mice .45

5.4.5 Formulation of the drugs for in vivo treatment............................................................ 45

5.4.6 Treatment of $\mathrm{Ptch}^{+/-}$mice with HhA and GDC-0941 ................................................. 45

5.4.7 Measurement of tumor growth using volumetric computer tomography (VCT) .............46

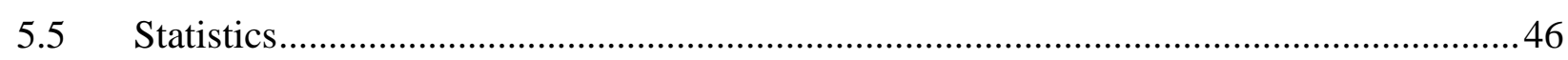

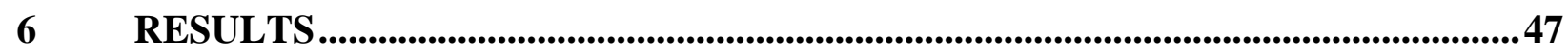

6.1 Effects of four SMO antagonists on RMS cell lines ................................................... 47

6.1.1 Modulation of gene expression by GDC-0449, LDE225, HhA and cyclopamine ........... 47

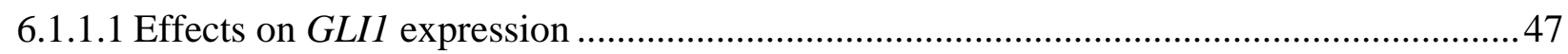

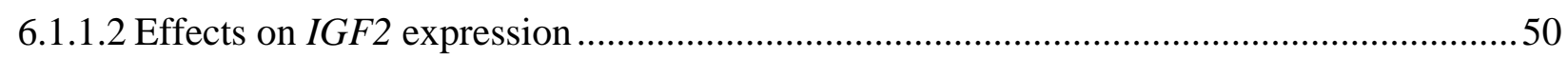

6.1.1.3 Effects on the expression of muscle differentiation markers .........................................52

6.1.2 Modulation of cellular viability, proliferation and apoptosis ........................................55

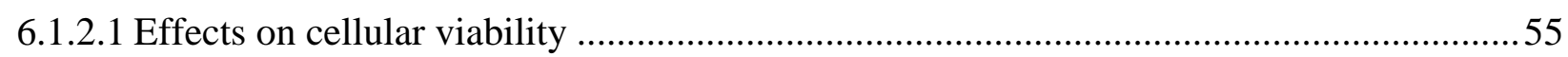

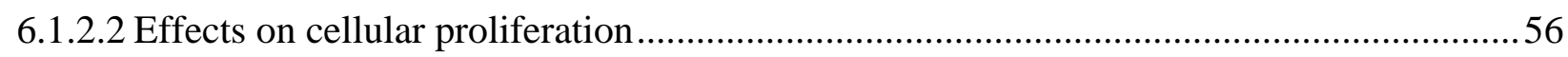

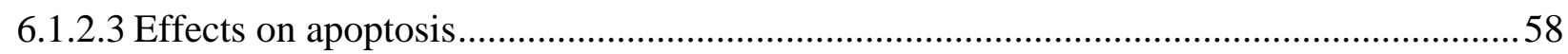

6.1.3 Effects on PI3K/AKT/mTOR signaling, on AMPK activity and on LC3-II expression...60

6.2 Effects of SMO antagonists in combination with PI3K/AKT/mTOR inhibitors on RMS

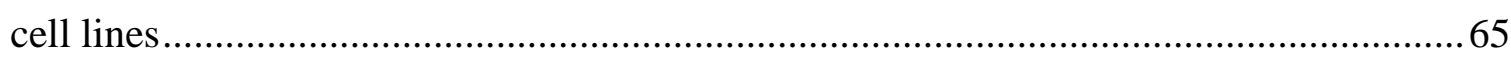

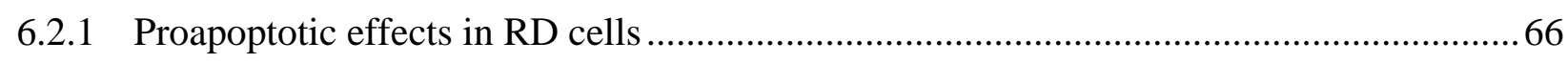

6.2.2 Effects on PI3K/AKT/mTOR signaling, on AMPK activity and on LC3-II protein levels in RD cells .68

6.2.3 Effects of HhA plus PI3K/AKT/mTOR inhibitors in RD, RUCH-2, RMS-13 and Rh41 cells.

6.2.3.1 Effects on $\mathrm{HH}$ signaling activity, IGF2 expression and muscle differentiation markers.. 71

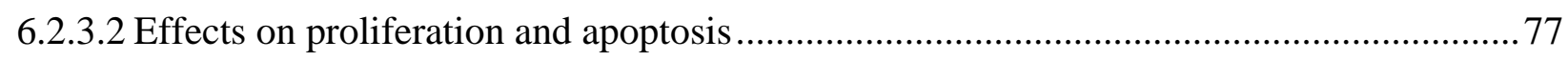

6.2.3.3 Effects on PI3K/AKT/mTOR signaling, on AMPK activity and on LC3-II expression... 80

6.3 In vivo effects of the SMO antagonist HhA plus PI3K inhibitor GDC-0941.................. 85

6.3.1 Effect of HhA plus GDC-0941 on tumor growth in $\mathrm{Ptch}^{+/-}$mice................................. 85

6.3.2 Immunohistochemical analysis of the proliferation marker Ki67 ................................. 86

7 DISCUSSION

7.1 Effects of four SMO antagonists on RMS cell lines .................................................. 90

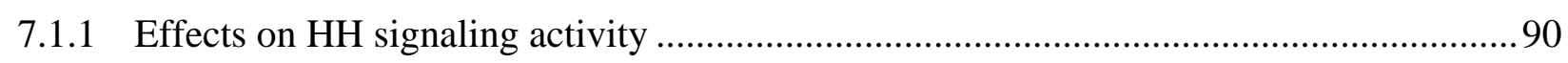

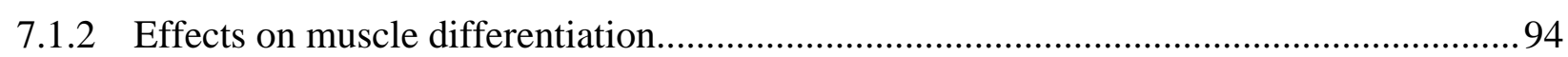




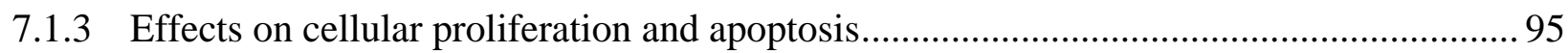

7.1.4 Effects on PI3K/AKT/mTOR signaling, on AMPK activity and on LC3-II expression .. 96

7.1.5 Overview on diverse and noncanonical effects of SMO inhibitors in RMS cells 98

7.2 Effects of combining SMO antagonists with PI3K/AKT/mTOR inhibitors in RMS ..... 100

7.2.1 Comparison of cooperative antitumor effects of SMO antagonists in combination with $\mathrm{PI} 3 \mathrm{~K} / \mathrm{AKT} / \mathrm{mTOR}$ inhibitors in RD cells

7.2.2 Detailed analyses of cooperative antitumoral effects of HhA plus PI3K/AKT/mTOR inhibitors 102

7.3 Outlook 107

8 REFERENCES 110

9 ABBREVIATIONS 123

10 ACKNOWLEDGEMENT 126 


\section{List of Figures}

Figure 1: Major components of canonical HH signaling. ......................................................

Figure 2: Aberrant activation of canonical HH signaling in cancer....................................... 7

Figure 3: Schematic representation of the PI3K/AKT/mTOR signaling cascade........................ 14

Figure 4: Interaction of $\mathrm{HH}$ and PI3K/AKT/mTOR signaling and associated inhibitors............ 19

Figure 5: Modulation of $\mathrm{HH}$ signaling activity by SMO antagonists. ...................................... 49

Figure 6: Modulation of IGF2 transcription by SMO antagonists..........................................51

Figure 7: Modulation of MYOD transcription by SMO antagonists.........................................53

Figure 8: Modulation of MYH1 transcription by SMO antagonists...........................................54

Figure 9: Cell viability/metabolic activity of RD cells after treatment with SMO antagonists....55

Figure 10: Modulation of proliferation by SMO antagonists................................................57

Figure 11: Determination of the cell numbers after treatment with SMO antagonists................58

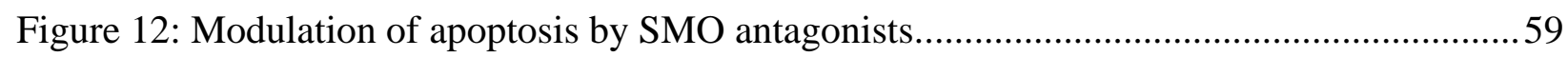

Figure 13: Impact of SMO antagonists on PI3K/AKT/mTOR signaling, phosphorylation of

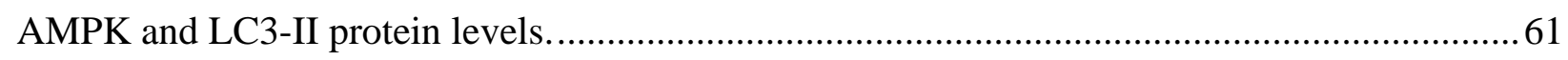

Figure 14: Impact of SMO antagonists on PI3K/Akt/mTOR signaling, phosphorylation of Ampk and Lc3-II protein levels in murine primary RMS cells.

Figure 15: Effects of SMO activation by SAG on PI3K/AKT/mTOR signaling, IGF2 and autophagy as well as on $\mathrm{HH}$ signaling activity.

Figure 16: Cooperative effects on apoptosis upon treatment with a PI3K/mTOR inhibitor plus DOX.

Figure 17: Regulation of apoptosis by SMO antagonists and/or PI3K/AKT/mTOR inhibitors...67

Figure 18: Effects of GDC-0449 and/or PI3K/AKT/mTOR inhibitors on PI3K/AKT/mTOR signaling, phosphorylation status of AMPK and LC3-II protein levels. . .68

Figure 19: Effects of LDE225 and/or PI3K/AKT/mTOR inhibitors on PI3K/AKT/mTOR signaling phosphorylation status of AMPK and LC3-II protein levels.

Figure 20: Effects of HhA and/or PI3K/AKT/mTOR inhibitors on PI3K/AKT/mTOR signaling, phosphorylation status of AMPK and LC3-II protein levels. 70 


\section{LIST OF FIGURES}

Figure 21: Modulation of $\mathrm{HH}$ signaling activity by HhA and/or PI3K/AKT/mTOR inhibitors. . 72

Figure 22: Modulation of IGF2 transcription by HhA and/or PI3K/AKT/mTOR inhibitors....... 73

Figure 23: Modulation of $M Y O D$ transcription by HhA and/or PI3K/AKT/mTOR inhibitors... 75

Figure 24: Modulation of $M Y H 1$ transcription by HhA and/or PI3K/AKT/mTOR inhibitors..... 76

Figure 25: Modulation of proliferation by HhA and/or PI3K/AKT/mTOR inhibitors................ 78

Figure 26: Modulation of apoptosis by HhA and/or PI3K/AKT/mTOR inhibitors.................... 79

Figure 27: Effects of HhA and/or PI3K/AKT/mTOR inhibitors on PI3K/AKT/mTOR signaling, phosphorylation status of AMPK and LC3-II protein levels in RD cells.

Figure 28: Effects of HhA and/or PI3K/AKT/mTOR inhibitors on PI3K/AKT/mTOR signaling, phosphorylation status of AMPK and LC3-II protein levels in RUCH-2 cells. 82

Figure 29: Effects of HhA and/or PI3K/AKT/mTOR inhibitors on PI3K/AKT/mTOR signaling, phosphorylation status of AMPK and LC3-II protein levels in RMS-13 cells. .83

Figure 30: Effects of HhA and/or PI3K/AKT/mTOR inhibitors on PI3K/AKT/mTOR signaling, phosphorylation status of AMPK and LC3-II protein levels Rh41cells. .84

Figure 31: Cooperative antitumoral effect of HhA and/or GDC-0941 on RMS growth in vivo. . 86 Figure 32: Analysis of the proliferation marker Ki67 in tumors derived from in vivo treatment. 87 Figure 33: Effects of HhA and/or GDC-0941 on PI3K/Akt/mTOR signaling, activation of Ampk and Lc3-II protein levels in murine primary RMS cells. 89 


\section{List of Tables}

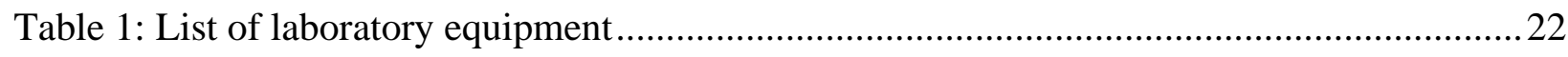

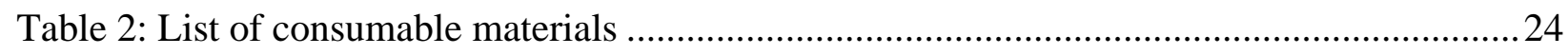

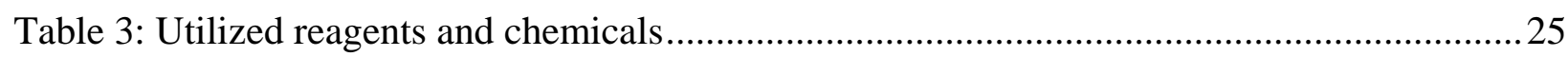

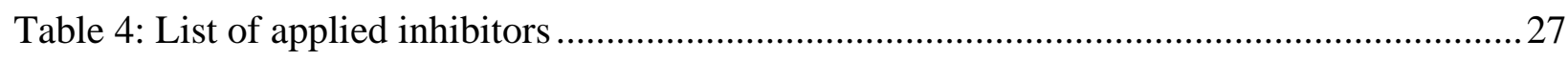

Table 5: Kits and ready-to-use reaction systems.......................................................... 28

Table 6: Buffers and solutions and their respective composition ..........................................28

Table 7: Media and reagents used for cell culture .......................................................... 31

Table 8: List of cell lines and their corresponding culture conditions .................................... 31

Table 9: Oligonucleotides for genotyping of $\mathrm{Ptch}^{+/-}$mice ....................................................... 32

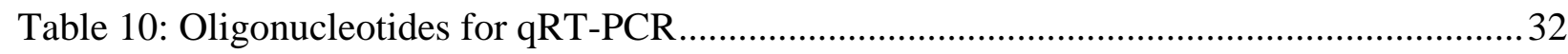

Table 11: Antibodies for western blot and immunohistochemistry ............................................33

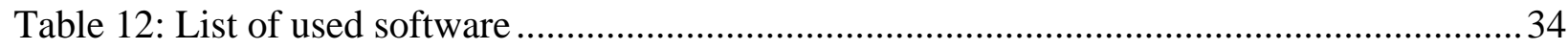

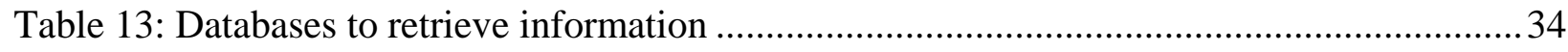

Table 14: Reaction mixture for genotyping of mouse tail gDNA .......................................... 37

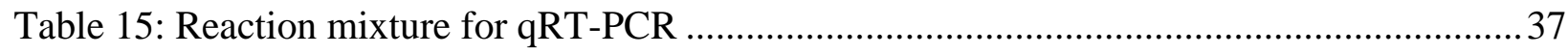

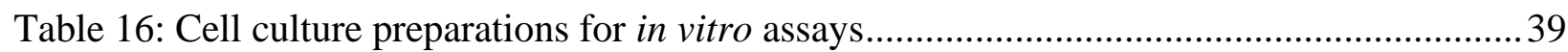





\section{SUMMARY}

Rhabdomyosarcoma (RMS) is the most common soft tissue sarcoma in children and occurs as two major subtypes; embryonal (ERMS) and alveolar RMS (ARMS). Therapies of RMS typically combine surgical excision or local irradiation with chemotherapy. However, the survival rate for metastatic disease is approximately $40 \%$ for ERMS and 10-30\% for ARMS. This highlights the need to evaluate novel therapeutic strategies that can improve the prognosis of these patients.

Like many other cancers, RMS show aberrant hedgehog $(\mathrm{HH})$ signaling activity. This implicates that RMS might be sensitive towards small molecule inhibitors directed against components of the $\mathrm{HH}$ pathway. Components of the canonical $\mathrm{HH}$ signaling cascade are the $\mathrm{HH}$ ligand, its receptor Patched (PTCH), the signaling partner of PTCH Smoothened (SMO) and the family of GLI transcription factors. An approved readout for active $\mathrm{HH}$ signaling is the transcriptional level of GLII.

Various drugs targeting the $\mathrm{HH}$ pathway have been developed and most of them inhibit the pathway at the level of SMO. In the first part of this thesis potential antitumoral effects of the SMO antagonists GDC-0449, LDE225, HhA and cyclopamine were compared using two ERMS (RD, RUCH-2) and two ARMS (RMS-13, Rh41) cell lines.

The data strongly suggest that GLII transcription and thus HH pathway activity in the used RMS cell lines is regulated independently of SMO by a noncanonical mechanism. Nevertheless, some SMO antagonists (i.e. HhA and LDE225) exerted strong antitumoral properties that were not associated with GLII expression. Interestingly, the mode of action of these compounds differed remarkably from each other. In addition, the observed effects were concentration dependent and the responsiveness of the RMS cell lines was heterogeneous. Thus, LDE225 and HhA induced strong proapoptotic and antiproliferative effects in most of the RMS cell lines. Moreover, the analysis revealed that LDE225 can activate caspase 3, HhA can block AKT activity and both drugs can stimulate autophagy (measured by LC3-II levels). GDC-0449 was less effective compared to LDE225 and HhA. It also paradoxically induced proliferation of some cell lines. This was similar for cyclopamine that induced cellular proliferation, never repressed $\mathrm{HH}$ signaling activity, showed no proapoptotic effects and activated AKT in most of the cell lines. In conclusion the data suggest that LDE225 and HhA are probably the most potent SMO inhibitors since they exerted antitumoral effects in almost all analyzed RMS cell lines. 
Besides HH signaling activity, RMS often show activation of the PI3K/AKT/mTOR pathway. Moreover, both pathways can interact with each other in several types of cancer. Hence, in the second part of this thesis SMO antagonists were combined with PI3K/AKT/mTOR inhibitors to investigate potential cooperative antitumoral effects. In order to block PI3K/AKT/mTOR signaling the dual PI3K/mTOR inhibitor PI103, the specific PI3K inhibitor GDC-0941, the pure AKT inhibitor MK-2206 and the mTOR inhibitors everolimus and rapamycin were applied.

The findings indicate that $\mathrm{HH}$ signaling in RMS cell lines is rather regulated in a noncanonical manner by the PI3K/AKT/mTOR pathway. Moreover, HhA was the most efficient SMO antagonist and showed several cooperative antitumoral effects upon combination with PI3K/AKT/mTOR inhibitors. The most promising combination was HhA plus GDC-0941 since this combination showed cooperative antiproliferative and proapoptotic effects in almost all cell lines. In addition, this combination was superior to single drug treatments in RMS bearing $\mathrm{Ptch}^{+/}$mice.

Taken together, the findings indicate that inhibition of $\mathrm{HH}$ signaling by a SMO antagonist plus an inhibitor of the PI3K/AKT/mTOR pathway may be an efficient strategy in order to improve therapy of RMS. 


\section{INTRODUCTION}

\subsection{Rhabdomyosarcoma}

Rhabdomyosarcoma (RMS) is the most common soft tissue sarcoma in children and represents the third most common extracranial solid childhood tumor after neuroblastoma and Wilms tumor (Kramer et al., 1983). It is a malignant tumor with a supposedly origin in the mesenchyme. Approximately $65 \%$ of all cases are diagnosed in children who are younger than six years. Common primary tumor sites are the head and neck region, the genitourinary tract and the extremities (Dagher and Helman, 1999).

RMS are subdivided by their histological features and genetic alterations into two major subtypes, the embryonal (ERMS) and the alveolar RMS (ARMS). ERMS is the more frequent subtype and accounts for two-thirds of cases. It predominantly occurs in infants and young children (Dagher and Helman, 1999; Merlino and Helman, 1999). This subtype is histologically characterized by spindle shaped cells with a stroma rich appearance. ERMS commonly show a loss of heterozygosity $(\mathrm{LOH})$ at the $11 \mathrm{p} 15.5$ locus, harboring the insulin-like growth factor 2 (IGF2). Due to loss of maternal and duplication of paternal chromosomal material this is commonly associated with overexpression of IGF2 (reviewed in (Merlino and Helman, 1999)). ARMS occur more frequently in adolescents and young adults. They show histological structures of small, densely packed cells lined up along spaces, which resembles pulmonary alveoli. ARMS can be further divided in fusion gene positive $(75 \%)$ and fusion gene negative (25\%) ARMS. Fusion gene positive ARMS harbor specific chromosomal translocations that lead to the fusion of the PAX3 or PAX7 gene with the forkhead transcription factor (FOXO1) (Dagher and Helman, 1999). In contrast ERMS are always fusion gene negative.

\subsection{Therapies of RMS}

Therapies of RMS are typically multidisciplinary and combine complete surgical excision for some patients, local irradiation for most patients and chemotherapy for all patients (Hawkins et al., 2014). Chemotherapy of RMS frequently involves treatment with vincristine, actinomycin D, doxorubicin (DOX), cyclophosphamide, ifosfamide or etoposide (Dagher and Helman, 1999). The application of a multi-agent chemotherapy with known activity commonly consists of 


\section{INTRODUCTION}

vincristine, actinomycin D plus cyclophosphamide (VAC), which is the gold standard procedure also for patients with higher risk (Dagher and Helman, 1999; Hawkins et al., 2013). The five year overall survival for RMS patients is approximately $73 \%$ for ERMS and $48 \%$ for ARMS (Ognjanovic et al., 2009). Nevertheless, the survival rate for metastatic ERMS is only $40 \%$ (Breneman et al., 2003). ARMS have a very poor prognosis when metastatic thus the survival rate is merely 10-30 \% (De Giovanni et al., 2009). This data indicate that although the current therapy opportunities for nonmetastatic RMS achieve acceptable outcomes, the perspective for already metastasized RMS stays very poor. However, the traditional chemotherapy for treatment of RMS has reached a plateau of efficacy (Egas-Bejar and Huh, 2014), in which treatment failure, toxicity and late effects including the formation of secondary cancers remain substantial (Dickerman, 2007). This highlights the need to evaluate novel therapies that can improve the prognosis of these patients. Therefore the development of new, targeted therapeutic strategies have been gained great interest (Egas-Bejar and Huh, 2014).

\subsection{The hedgehog signaling pathway}

The hedgehog $(\mathrm{HH})$ signaling pathway was initially discovered in 1980 as a regulatory pathway of embryonic patterning in Drosophila melanogaster (Nusslein-Volhard and Wieschaus, 1980). The name of the pathway is derived from the hedgehog like appearance of the Hh mutant fruit fly larvae.

In mammals, HH signaling pathway also plays a substantial role in embryonic development, cell fate decisions and tissue growth. Moreover, it controls cell proliferation in many embryonic and adult tissues (reviewed in (Jiang and Hui, 2008)). HH signaling is generally quiescent in the adult organism except for its involvement in the regulation of cell differentiation, stem cell maintenance, tissue homeostasis and repair (reviewed in (Petrova and Joyner, 2014)). Furthermore novel findings linked the $\mathrm{HH}$ pathway also to the regulation of metabolic homeostasis (Teperino et al., 2014).

\subsubsection{Canonical HH signaling}

The major components of the canonical HH signaling cascade comprise the ligand $\mathrm{HH}$, the transmembrane receptor Patched $(\mathrm{PTCH})$, the transmembrane signal transducer Smoothened (SMO) and the family of glioma-associated oncogene (GLI) transcription factors. A very simplified diagram of the pathway is shown in Figure 1. In mammals three HH ligands exist, 
which are sonic hedgehog $(\mathrm{SHH})$, desert hedgehog and indian hedgehog, each with distinct temporal and spatial expression patterns (Ryan and Chiang, 2012). In the absence of the ligand $\mathrm{HH}, \mathrm{PTCH}$ inhibits SMO resulting in the formation of transcriptional repressor forms of GLI and thus inactivation of the pathway (Figure $1 \mathrm{~A}$ ). HH pathway activation is induced by binding of $\mathrm{HH}$ to its receptor PTCH. Thereby HH inhibits PTCH. This suspends the inhibition of SMO (Figure 1 B). After a series of poorly understood events that include SMO trafficking to the primary cilia, SMO activates the GLI transcription factors GLI2 and GLI3. Consequently, activated GLI2 and GLI3 induce the expression of several downstream target genes. Besides $\mathrm{PTCH}$, another negative regulator of the HH pathway is suppressor of fused (SUFU), which sequesters GLI transcription factors in the cytoplasm and thus represses their transcriptional activity (reviewed in (Robbins et al., 2012; Rohatgi and Scott, 2007)).

A

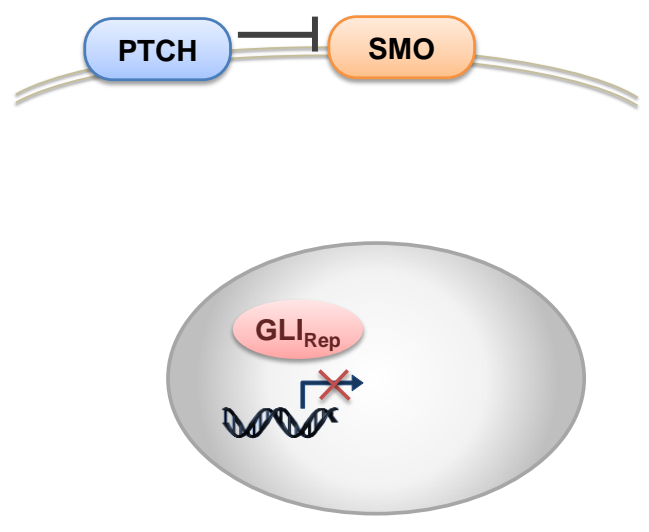

\section{B}

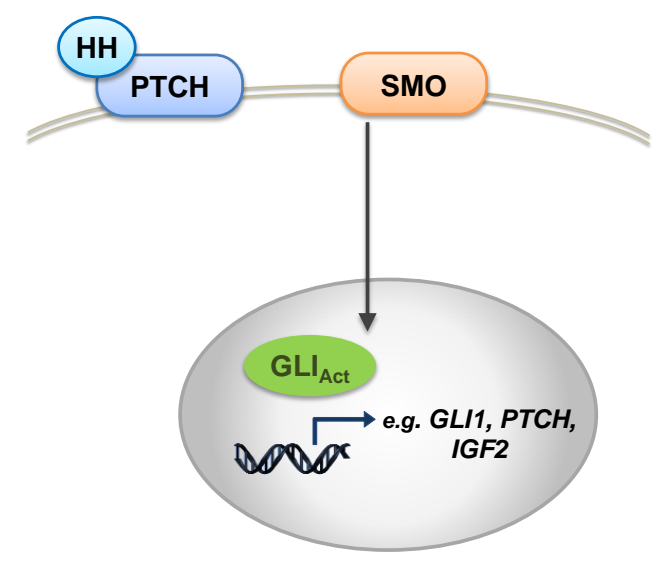

Figure 1: Major components of canonical HH signaling. The four major components of canonical HH signaling are illustrated; the ligand hedgehog $(\mathrm{HH})$, its transmembrane receptor Patched $(\mathrm{PTCH})$, the transmembrane signal transducer Smoothened (SMO) and glioma-associated oncogene (GLI) transcription factors. (A) In the absence of the ligand HH, PTCH inhibits SMO. This keeps the pathway in its inactive state due to the cleavage of the GLIs into their repressor forms $\left(\mathrm{GLI}_{\mathrm{Rep}}\right)$, which inhibits target gene expression. (B) Activation of the pathway is induced by binding of HH to its receptor PTCH, which suspends the inhibition of SMO. Subsequently SMO stabilizes the GLI activator forms $\left(\mathrm{GLI}_{\mathrm{Act}}\right)$, which translocate in the nucleus where they induce expression of $\mathrm{HH}$ target genes.

Besides other targets, the $\mathrm{HH}$ signal triggers the expression of GLI1, which amplifies $\mathrm{HH}$ signaling. Another important downstream target of the pathway is $\mathrm{PTCH}$, which in contrast to GLI1 generates a negative feedback loop to the canonical HH pathway. Together GLII and PTCH mRNA transcription levels are considered as reliable markers for the pathway's activity 


\section{INTRODUCTION}

(reviewed in (Scales and de Sauvage, 2009)). Finally, also the expression of IGF2 can be triggered by the HH signal (Corcoran et al., 2008; Hahn et al., 2000). Gene expression analysis revealed that IGF2 is frequently overexpressed in RMS and plays also a key role in the formation, proliferation, growth and metastasis of RMS (reviewed in (Martins et al., 2011)).

Nevertheless, the precise interplay of the pathway components is still poorly defined, e.g. how the signal is transduced between SMO and GLI proteins. Moreover, the mechanism how PTCH regulates SMO has still not been revealed (Jiang and Hui, 2008). However, a direct physical interaction of PTCH and SMO can be excluded (Denef et al., 2000; Taipale et al., 2002). SMO activity is therefore commonly thought to be regulated by endogenous small molecules. This hypothesis is supported by the discovery of various small molecule drugs that bind SMO and either activate or inhibit its function (Rohatgi and Scott, 2007).

\subsubsection{Canonical HH signaling in cancer}

$\mathrm{HH}$ signaling activation results in the expression of cell specific transcription factors which mediate different effects including developmental fate responses (McMahon et al., 2003), upregulation of $\mathrm{N}$-myelocytomatosis oncogene (N-Myc) and D-type cyclins that cause proliferation (Duman-Scheel et al., 2002; Kenney et al., 2003; Kenney and Rowitch, 2000), stimulation of antiapoptotic proteins like B-cell lymphoma $2(\mathrm{Bcl} 2)$, that mediate cell survival (Bigelow et al., 2004; Regl et al., 2004) and regulation of angiogenesis by vascular endothelial growth factor (Pola et al., 2001). Therefore, it is not surprising that dysregulation of $\mathrm{HH}$ signaling is relevant for tumor formation and progression of a multiplicity of cancers (Scales and de Sauvage, 2009).

In several cancers a constitutive activation of $\mathrm{HH}$ signaling is observed. This can be mediated by ligand dependent activation due to the overexpression of the HH ligands (Oro et al., 1997) or by ligand independent activation, due to mutational inactivation of the tumor suppressors PTCH or SUFU (Goodrich et al., 1997; Hahn et al., 1996; Taylor et al., 2002), activating mutations of SMO (Lam et al., 1999) or overexpression of GLI transcription factors (Nilsson et al., 2000).

Types of cancers which showed ligand dependent HH signaling activity (Figure 2 A) generally do not harbor driver mutations of $P T C H$ or SMO and found e.g. in cancers of the breast, the gastrointestinal tract, the prostate as well as in hematological malignancies and gliomas (Beachy et al., 2004; Lindemann, 2008; Mukherjee et al., 2006; Ruiz i Altaba et al., 2007). Nevertheless, the relevance of a ligand dependent mechanisms as the major driver of cancer growth is less 
clear because of multiple influencing factors including the tumor stroma and interactions with other oncogenic pathways (reviewed in (Amakye et al., 2013)).

Ligand independent pathway activation (Figure 2 B) due to mutations of $\mathrm{HH}$ pathway components have been associated with basal cell carcinoma (BCC) and medulloblastoma (MB) (Wicking et al., 1999). Indeed nearly all sporadic BCC are caused by inactivating mutations of PTCH (more than $90 \%$ ) or activating mutations in SMO and over $25 \%$ of sporadic MB are related to mutations in either PTCH or SUFU (reviewed in (Scales and de Sauvage, 2009)).
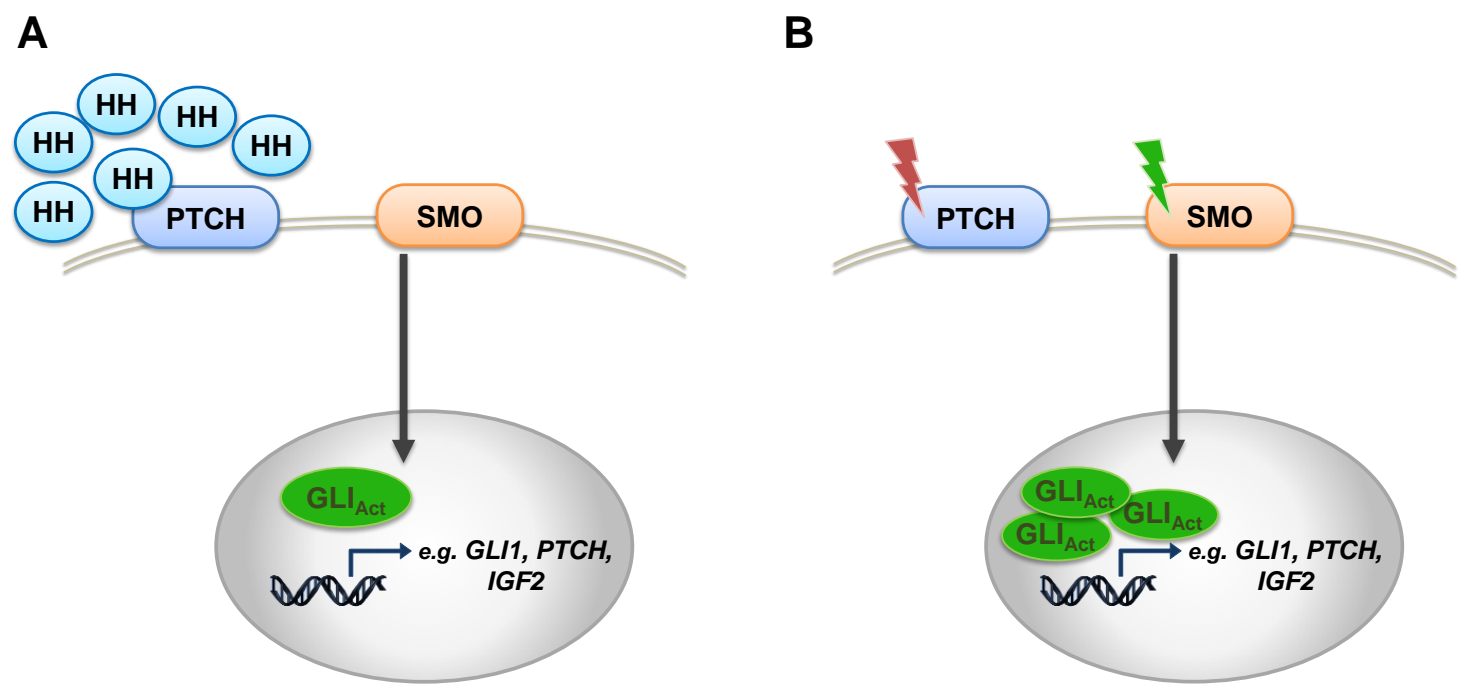

Figure 2: Aberrant activation of canonical HH signaling in cancer. Pathological activation of canonical HH signaling in cancer can be mediated by two key mechanisms. (A) Ligand dependent pathway activation is induced by overexpression of the HH ligand. (B) Ligand independent cancers can harbor inactivating mutations in PTCH (red flash), activating mutations in SMO (green flash) or overexpress the GLI transcription factors $\left(\mathrm{GLI}_{\mathrm{Act}}\right.$ ), resulting in $\mathrm{HH}$ pathway activation even in the absence of the ligand $\mathrm{HH}$.

\subsubsection{Noncanonical HH signaling}

In the last years, numerous evidences indicated that components of the canonical HH pathway also modulate cellular processes independently of the canonical $\mathrm{HH}$ axis. For instance, in the absence of HH, PTCH seems to be involved in cell cycle regulation and induction of apoptosis autonomously of GLI (Barnes et al., 2001; Thibert et al., 2003). Moreover SMO has been implicated in cytoskeletal rearrangement in a GLI independent manner (reviewed in (Robbins et al., 2012)) and signaling of the second messenger $\mathrm{Ca}^{2+}$ (Belgacem and Borodinsky, 2011). Thus, noncanonical HH signaling can be described as signaling derived from PTCH or SMO but independently of GLI transcription factors (reviewed in (Robbins et al., 2012)). 
On the other hand noncanonical $\mathrm{HH}$ signaling can also be defined by SMO independent activation of GLI transcription factors since in several cancers GLI transcription factors are regulated by multiple oncogenic pathways, such as the RAS/RAF/MEK pathway in pancreatic cancer and melanoma (Ji et al., 2007; Nolan-Stevaux et al., 2009; Stecca et al., 2007) and the phosphatidylinositol-3-kinase (PI3K)/AKT/mammalian target of rapamycin (mTOR) pathway in glioblastoma and esophageal adenocarcinoma (EAC) (Filbin et al., 2013; Stecca et al., 2007; Wang et al., 2012). Moreover, GLI transcription factors are regulated through tumor suppressors like phosphatase and tensin homolog deleted on chromosome 10 (PTEN) and transformation related protein 53 (p53) (reviewed in (Stecca and Ruiz, 2010)).

\subsection{HH signaling in RMS}

The initial link between HH signaling and cancer formation (e.g. RMS) was found in patients suffering from an inherited haploinsufficiency of PTCH (Gorlin syndrome or basal cell nevus syndrome) (Hahn et al., 1996; Johnson et al., 1996). Besides developmental defects, these patients show predisposition to BCC, MB and RMS (Gorlin, 1987; Hahn et al., 1999). This provided first evidence of an existing correlation between constitutive activated $\mathrm{HH}$ signaling and RMS. In addition, $\mathrm{Ptch}^{+/-}$mice spontaneously develop RMS that resembles the embryonal subtype in humans. This is based on histological appearance and on overexpression of the global HH markers genes Gli1, Ptch as well as Igf2 (Hahn et al., 2000; Hahn et al., 1998), which is also highly expressed in human ERMS (Merlino and Helman, 1999).

However, the role of $P T C H$ mutations in sporadic human RMS is less clear, since several studies screening for $P T C H$ or SMO mutations in human RMS generated conflicting outcomes. For instance in deoxyribonucleic acid (DNA) sequence analyses of 14 RMS no point mutation or deletion in the PTCH region and only one case of $\mathrm{LOH}$ at the PTCH locus was detected. This indicates that sporadic RMS cannot explicitly linked to HH pathway mutations (Calzada-Wack et al., 2002). On the other hand, in one-third of ERMS genomic imbalances including loss of the chromosomal region 9q22 (which includes the PTCH gene) or amplification of the GLI1 gene have been found (Bridge et al., 2000; Merlino and Helman, 1999). Moreover, several studies revealed that both ERMS and ARMS express elevated PTCH and GLII mRNA levels compared to the corresponding normal tissue (Tostar et al., 2006) and also display increased GLI1 protein levels (Ragazzini et al., 2004). Furthermore, ERMS and fusion gene negative ARMS are characterized by higher HH signaling activity as shown by GLII and PTCH expression when compared to fusion gene positive ARMS (Pressey et al., 2011; Zibat et al., 2010). These findings 
indicate that alteration in PTCH and GLII expression levels and consecutive overexpression of HH target genes might indeed play a role in sporadic RMS.

Although the mechanisms responsible for HH pathway activation in RMS remain elusive (i.e. no clear evidence for activating mutations in the $\mathrm{HH}$ pathway have been identified) these findings strongly implicate that RMS might be sensitive towards a targeted therapy using small molecule $\mathrm{HH}$ inhibitors directed against components of the HH pathway.

\subsection{HH inhibitors - SMO antagonists}

In the last years several drugs targeting the $\mathrm{HH}$ pathway have been developed for the treatment of patients with diverse cancers. Thereby compound discovery has been predominantly focused on drugs that bind and block SMO in order to inhibit the HH signaling pathway (SMO antagonists or SMO inhibitors). The impact of many SMO antagonists have been already analyzed in phase I/II clinical trials with promising results for BCC and MB treatment (reviewed in (Amakye et al., 2013)). However, only a couple of RMS patients have been included in clinical trials testing the potential antitumor effects of SMO inhibitors. These studies were recently completed and the results are eagerly awaited (reviewed in (Amakye et al., 2013)).

Although clinical trials using SMO antagonists provided promising results, in tumors with $\mathrm{HH}$ pathway activation downstream of SMO (e.g. due to loss of SUFU, amplification of GLI or noncanonical GLI signaling) these compounds are ineffective. Hence, inhibitors targeting GLI transcription factors have been established, but they have not advanced to clinical development so far (Amakye et al., 2013; Ng and Curran, 2011).

\subsubsection{Cyclopamine - a prototype of SMO inhibitors}

Cyclopamine - the first described small molecule inhibitor of the HH pathway (Cooper et al., 1998) - is a natural alkaloid of the corn lily Veratrum californicum. It was identified as a teratogen responsible for developmental defects in lambs (e.g. cyclopia and holoprosencephaly) (Binns et al., 1968) due to inhibition of the $\mathrm{HH}$ signaling pathway by direct binding to SMO (Chen et al., 2002a). This binding stimulates the translocation of SMO to the primary cilium without activation of GLIs (Wang et al., 2009). The synthetically generated SMO antagonists SANT1 and SANT2 also bind to the cyclopamine binding pocket but in contrast to cyclopamine abrogate the ciliary translocation of SMO (Wang et al., 2009). Therefore, it is thought that 


\section{INTRODUCTION}

activation of SMO represents a multistep process, in which cyclopamine prevents its conversion from a ciliary inactive to a ciliary active conformation (Rohatgi et al., 2009).

Since cyclopamine has poor oral solubility, limited potency, nonspecific toxicity and chemical instability it is not suitable as a therapeutic agent in humans (Lipinski et al., 2008). However, it has been widely used for in vitro and in vivo experiments to investigate the effects of $\mathrm{HH}$ pathway inhibition in several biological processes. In vitro studies reported that cyclopamine inhibits the proliferation of a variety of cancer cell lines e.g. from breast (Kubo et al., 2004), prostate (Sanchez et al., 2004), colorectal and pancreas tumors (Qualtrough et al., 2004; Thayer et al., 2003) as well as of MB cell lines (Berman et al., 2002; Sanchez and Ruiz i Altaba, 2005). These antitumoral effects have been also confirmed in the $P t c h 1^{+/} p 53^{-/-}$mouse model for MB (Sanchez and Ruiz i Altaba, 2005) and in xenograft models using e.g. prostate carcinoma cells (Karhadkar et al., 2004), glioblastoma cells (Clement et al., 2007), tumor cells of the digestive tract (Berman et al., 2003) and the pancreas (Feldmann et al., 2007; Thayer et al., 2003). Nevertheless, the evaluation of cyclopamine as an effective inhibitor of RMS revealed that it inhibited Hh signaling and proliferation of in vitro cultured primary RMS cells. Although Hh signaling was partially suppressed also in RMS in vivo, cyclopamine failed to inhibit the in vivo growth of RMS in $\mathrm{Ptch}^{+/-}$mice and rather seemed to enhance the growth of the tumors (Ecke et al., 2008).

\subsubsection{GDC-0449, LDE225 and HhA - more potent derivatives}

A variety of orally bioavailable SMO antagonists with increased potency have been synthesized and many of them are structurally unrelated to cyclopamine. SMO inhibitors that are currently investigated in clinical trials in a range of advanced cancers are GDC-0449 (vismodegib; Curis/Roche), LDE225 (sonidegib; Novartis), BMS-833923 (Bristol-Myers Squibb), PF04449913 (Pfizer) and LY2940680 (Eli Lilly) (reviewed in (Amakye et al., 2013)). Several of these compounds have triggered tumor responses particularly in patients with BCC and MB, which harbor PTCH mutations, i.e. mutations upstream of SMO (Amakye et al., 2013; LoRusso et al., 2011; Rudin et al., 2009; Sekulic et al., 2012).

GDC-0449 represents the frontrunner of SMO inhibitors used in the clinics and shows attractive preclinical pharmacokinetic and drug metabolism properties in rat, mouse, dog and monkey (Wong et al., 2009). GDC-0449 was approved in 2012 by the US Food and Drug Administration for the treatment of locally advanced BCC and metastatic BCC (Rudin, 2012) due to tumor response rates of $43 \%$ and $30 \%$, respectively (Sekulic et al., 2012). In addition, GDC-0449 
reduced the numbers of new developing $\mathrm{BCC}$ and the size of existing $\mathrm{BCC}$ of Gorlin syndrome patients. This effect was associated with a $90 \%$ decrease in HH signaling activity and reduced tumor proliferation, without affecting apoptosis rate (Tang et al., 2012). Tumor responses with GDC-0449 have also been observed in MB patients (Amakye et al., 2013) and in mouse MB allografts from $\mathrm{Ptch}^{+/}$mice (Robarge et al., 2009). Actually, GDC-0449 represents the most investigated SMO inhibitor in the clinics and is also used in clinical trials of advanced solid tumors including few cases of adult RMS patients, but currently with unclear outcome (Amakye et al., 2013).

Another SMO inhibitor used in this thesis is LDE225, which is a potent and selective SMO antagonist from a novel structural class (Pan et al., 2010). Similarly to GDC-0449, LDE225 shows antitumoral activity and suppression of $\mathrm{HH}$ signaling in $\mathrm{MB}$ and $\mathrm{BCC}$ patients (Amakye et al., 2013). LDE225 was approved in July 2015 by the US Food and Drug Administration for the treatment of locally advanced BCC. Moreover, LDE225 already passed a phase II clinical trials for children with MB or other solid tumors, including a few cases of RMS. Currently available preliminary data show that LDE225 is well tolerated by the patients and shows promising efficacy in MB patients (Amakye et al., 2013). Many preclinical studies using LDE225 have been published. These studies show that LDE225 reduces GLI1 and GLI2 protein levels in human renal cell carcinoma (RCC) cell lines and possesses antitumoral effects in RCC tumor xenografts (D'Amato et al., 2014). Furthermore, LDE225 efficiently inhibits GLI1 expression and growth of human melanoma cell lines and blocks the growth of melanoma xenografts in vivo (Jalili et al., 2013). Tumors of different PTCH mutant MB xenograft models are also responsive to LDE225 treatment as well as osteosarcoma bearing mice (Kool et al., 2014; Paget et al., 2012).

HhA (HhAntag691) is a benzimidazole, which was identified by high-throughput cell based screenings of a collection of small molecules (Romer et al., 2004). HhA provides the most convincing preclinical data on the efficacy of SMO inhibitors in a $\mathrm{Ptch}^{+/} \mathrm{p} 53^{-/-}$mouse model of spontaneously developing MB (Romer et al., 2004). Oral delivery of HhA to this mouse model decreased the Glil expression and inhibited tumor growth by reducing cell proliferation and increasing apoptosis to the point of complete eradication of large MB (Romer et al., 2004). Preclinical studies with HhA predicted potential responses of BCC and $\mathrm{HH}$ pathway activated $\mathrm{MB}$ in the clinics. This led to the inclusion of $\mathrm{MB}$ patients in initial clinical trials $(\mathrm{Ng}$ and Curran, 2011). However, HhA subsequently was shown to have an inadequate hepatic clearance. Therefore it is suboptimal as a therapeutic drug in humans (Dijkgraaf et al., 2011). 


\subsubsection{Side effects and toxicities of SMO antagonists}

SMO inhibitors have been shown to be generally well tolerated as side effects are fairly modest in adult patients. Clinical data of GDC-0449 and LDE225 reported that the most common toxicities comprise taste alteration, nausea, anorexia, weight loss, alopecia, muscle spasms and fatigue (reviewed in (Amakye et al., 2013)). Although moderate, the chronic nature of the side effects of SMO inhibitors led to discontinuation of therapy in $50 \%$ of patients in GDC-0449 trials of BCC treatment. Since this cancer is not typically life threatening, probably much self-motivation is needed for compliance to SMO inhibitor therapy (Sekulic et al., 2012; Tang et al., 2012). Whereas SMO inhibitors may be a good treatment option in adults with $\mathrm{HH}$ associated cancers, this may be different in children. Thus, preclinical data described dramatic and permanent bone defects in young mice treated with HhA (Kimura et al., 2008). In addition, SMO inhibitors may affect the proper development of many other organs such as the brain. This would be especially deleterious in young patients and may hamper the application of these drugs in pediatric tumors such as RMS.

\subsubsection{Mechanisms of acquired resistance to SMO inhibitors}

Treatment with SMO inhibitors can lead to the occurrence of acquired tumor resistance caused by three different possible mechanisms: i) secondary mutation in SMO; ii) amplification of downstream $\mathrm{HH}$ target genes; and/or iii) compensatory upregulation of noncanonical $\mathrm{HH}$ signaling.

In 2009, one report described a considerable response of a patient with a PTCH mutant MB after two months GDC-0449 treatment, which relapsed just one month later (Rudin et al., 2009). Subsequently, a secondary mutation in SMO D473H was identified in the tumor material as the reason of the acquired resistance (Yauch et al., 2009). Interestingly, the mutation did not affected $\mathrm{HH}$ signaling activity but impaired the binding of GDC-0449 to SMO and consequently the ability to suppress the pathway. This resulted in reactivation of $\mathrm{HH}$ signaling and restoration of tumor growth. Moreover, a matching mutation in the same amino acid of Smo was found in MB of $P t c h 1^{+/}{\mathrm{p} 53^{-/-}}$mice, which similarly responded to GDC-0449 treatment and relapsed later (Yauch et al., 2009). LDE225 treatment can also lead to secondary resistance mutations as described for MB of Ptch mouse models (Buonamici et al., 2010), but they differed from the mutations found in GDC-0449 resistant tumors. Indeed, several SMO inhibitors have variable potency in blocking the activity of different SMO mutational variants (Dijkgraaf et al., 2011; Tao et al., 2011) as shown for GDC-0449 which had a weak potency against the Smo E518K and 
Smo D473H variants, whereas HhA was essentially equipotent against all Smo alleles (Dijkgraaf et al., 2011).

Amplification of Gli2 as a further mechanism of resistance to SMO inhibition was initially identified in MB of $\mathrm{Ptchl}^{+/} \mathrm{p} 53^{-/-}$mice after treatment with GDC-0449 or LDE225, respectively (Buonamici et al., 2010; Dijkgraaf et al., 2011). It was associated with increased Gli2 mRNA expression, which mediated tumor growth in a Smo independent manner (Buonamici et al., 2010).

Finally, acquired resistance to treatment with SMO inhibitors can also depend on activation of noncanonical Hh signaling as initially found in LDE225 treated allografted $P t c h 1^{+-} p 53^{-/-} \mathrm{MB}$ cells. Although LDE225 inhibited Hh signaling and induced tumor regressions, resistance was observed during the course of treatment. LDE225 resistant tumors showed an increased PI3K signaling activity (Buonamici et al., 2010). However, the molecular effects that led to the compensatory adaptation due to activation of PI3K pathway are unknown, but interestingly upregulation of the PI3K pathway was also detected in tumors with and without Gli2 amplification (Buonamici et al., 2010).

Thus, targeted therapy with SMO antagonists combined with inhibitors of the PI3K/AKT/mTOR pathway might be an attractive treatment option for RMS of young children. The combination of these drugs could potentially help to avoid side effects of SMO inhibitors by lowering the doses of the SMO inhibitors in combination treatment and to specifically circumvent a possible induction of PI3K signaling.

\subsection{The PI3K/AKT/mTOR pathway}

The PI3K/AKT/mTOR signaling pathway is important in many aspects of growth and cell survival and regulates various cellular processes, such as proliferation, apoptosis, cytoskeletal rearrangement and metabolism. Under physiological conditions this pathway is activated by many extracellular stimuli e.g. insulin, growth factors and chemokines (Lim et al., 2015). The $\mathrm{PI} 3 \mathrm{~K} / \mathrm{AKT} / \mathrm{mTOR}$ signaling cascade is schematically illustrated in Figure 3.

One of the main components displays PI3K, which is a heterodimeric lipid kinase consisting of a regulatory and a catalytic subunit that are encoded by different genes. Binding of certain ligands to receptor tyrosine kinases (RTK), such as the IGF1 and IGF2 receptor (IGF1R, IGF2R) or the epidermal growth factor receptor (EGFR), leads to autophosphorylation and activation of these receptors. Subsequently, the regulatory subunit of PI3K binds (together with or without adaptors) to the receptors. This results in recruitment of PI3K to the plasma membrane where it 


\section{INTRODUCTION}

phosphorylates its substrate, phosphatidylinositol 4,5-bisphosphate (PIP2) to produce the second messenger phosphatidylinositol 3,4,5-trisphosphate (PIP3). PIP3 then distributes intracellular signaling by direct contact with pleckstrin homology $(\mathrm{PH})$ domains of various signaling proteins such as e.g. phosphoinositide dependent kinase 1 (PDK1) and AKT (or protein kinase B, PKB) that are subsequently recruited to the inner side of the plasma membrane. PDK1 partially activates AKT by phosphorylation of threonine 308 (Figure 3) (reviewed in (Courtney et al., 2010; Vivanco and Sawyers, 2002)).

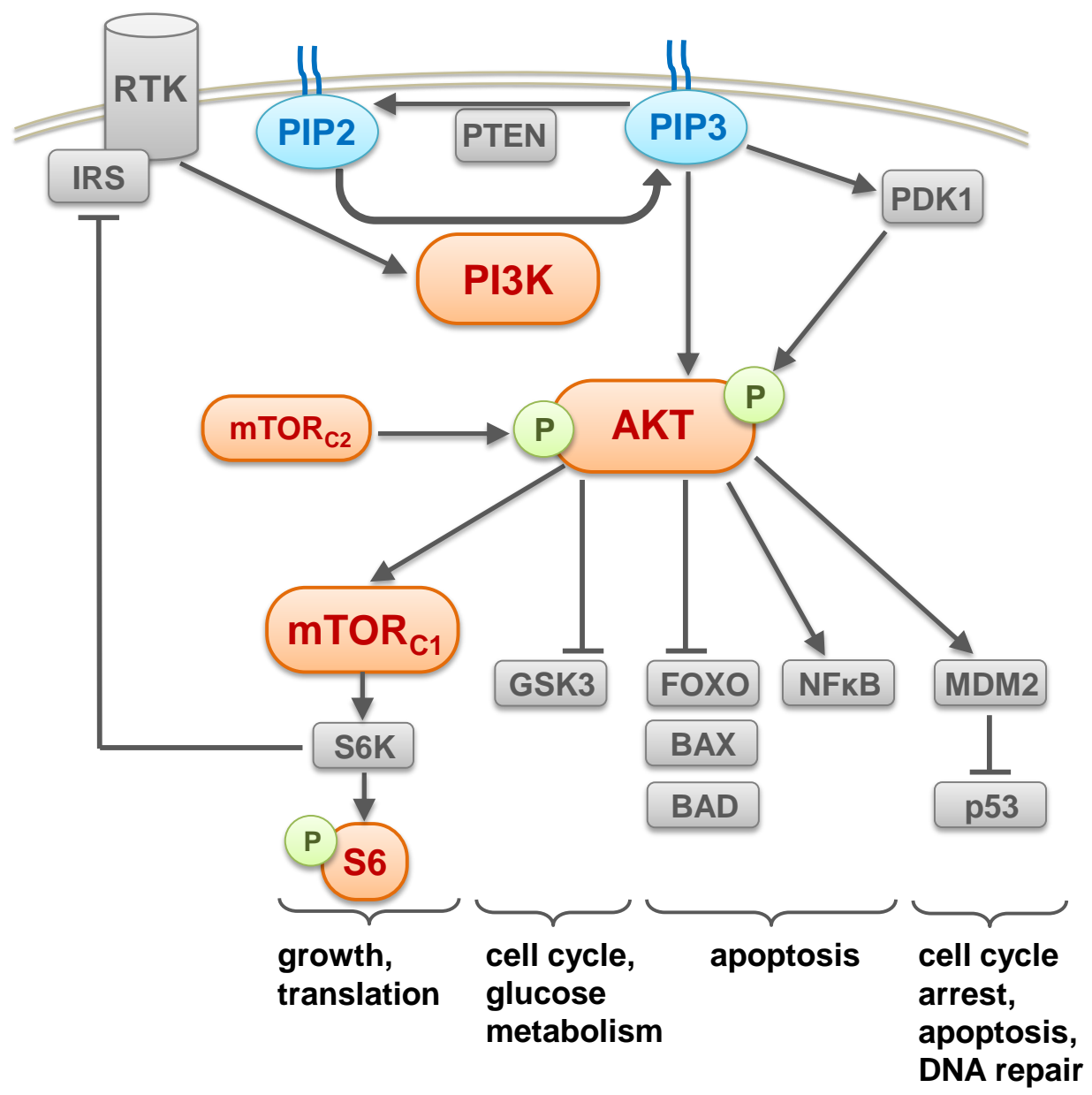

Figure 3: Schematic representation of the PI3K/AKT/mTOR signaling cascade. In response to extracellular stimuli, PI3K is activated by receptor tyrosine kinases (RTK) and phosphorylates PIP2 to generate PIP3. Consequently, AKT is activated by phosphorylation of threonine 308 and serine 473. Subsequently, AKT activates its downstream effectors. For a more detailed description please see the text.

$\mathrm{AKT}$ is a serine/threonine kinase that controls many biological processes by phosphorylation of its targets. For instance, AKT stimulates cell survival by inhibiting the proapoptotic Bcl-2 family members Bcl-2-associated death promoter (BAD) and Bcl-2-associated X protein (BAX). AKT 
also impairs negative regulation of the transcription factor nuclear factor kappa-light-chainenhancer of activated $\mathrm{B}$ cells $(\mathrm{NF \kappa B})$, thereby enhancing transcription of antiapoptotic and prosurvival genes. Moreover, phosphorylation of murine double minute 2 (MDM2) by AKT antagonizes p53 mediated apoptosis. Furthermore, negative regulation of FOXO by AKT leads to reduced synthesis of cell death promoting proteins. AKT also modulates proliferation by inhibition of glycogen synthase kinase 3 (GSK3), which prevents cyclin D1 degradation (reviewed in (Hennessy et al., 2005; Vivanco and Sawyers, 2002)). Inactivated GSK3 also blocks glycogen synthesis, which contributes to the stimulation of glycogen and protein synthesis and thus influences metabolism (Cohen and Frame, 2001).

Importantly, AKT activates the mTOR-containing protein complex $\mathrm{mTOR}_{\mathrm{Cl}}$, leading to increased protein synthesis by phosphorylation of ribosomal S6 protein (S6) and eukaryotic translation initiation factor $4 \mathrm{E}$-binding protein 1 (4EBP1). Whereas $\mathrm{mTOR}_{\mathrm{C} 1}$ transmits signals from PI3K/AKT cascade, the second mTOR complex mTOR $_{\mathrm{C} 2}$ contributes to complete AKT activation by phosphorylation on serine 473 (Sarbassov et al., 2005). The mTOR $_{\mathrm{Cl}}$ downstream target S6 kinase (S6K) blocks ligand mediated PI3K activation by inhibiting the adaptor protein insulin receptor substrate (IRS) and thereby negatively feeds back to diminish PI3K activation (Carracedo and Pandolfi, 2008). Moreover, the phosphatase PTEN antagonizes PI3K signaling by dephosphorylating of PIP3 to PIP2, hence acting as a tumor suppressor (reviewed in (Courtney et al., 2010; Vivanco and Sawyers, 2002)).

\subsubsection{PI3K/AKT/mTOR signaling in RMS}

The PI3K/AKT/mTOR pathway is dysregulated in a wide spectrum of human cancers including those of the breast, ovarian and prostate, glioblastoma, colorectal cancer (CRC), hematological malignancies as well as sarcomas (reviewed (Lim et al., 2015)). Several studies show that numerous components of the PI3K/AKT/mTOR pathway are affected in tumors by e.g. amplification, mutation and translocation. The constitutive activation of this pathway not only leads to cellular growth and survival but also to metastatic ability and to therapy resistance of the tumors (reviewed in (Courtney et al., 2010; Hennessy et al., 2005)).

The PI3K/AKT/mTOR signaling pathway also plays a crucial role in RMS. Activation of the PI3K/AKT/mTOR pathway, demonstrated by AKT phosphorylation (pAKT), was observed in human RMS and high levels of activated AKT were related to poor overall and disease free survival in these patients (Petricoin et al., 2007). Moreover, tissue microarray analyses revealed that $82.5 \%$ of RMS stained positive for pAKT (Renshaw et al., 2013). RMS had also been 
related to enhanced expression of IGF pathway proteins including IGF1, IGF2, IGF binding proteins 2 (IGFBP2) and to expression of IGF1R and IGF2R (Makawita et al., 2009). Additionally, RMS show increased levels of EGFR and ErbB-2 without evidence of EGFR or ErbB-2 amplifications or mutations in the EGFR tyrosine kinase domain (Ganti et al., 2006). Hence PI3K/AKT/mTOR pathway activation in RMS could also be promoted by the expression of these stimuli. Moreover, RMS from $\mathrm{Ptch}^{+/-}$mice show increased activity of Akt. Since these tumors show high Igf2 transcript levels (Kappler et al., 2003) activation of the PI3K/Akt/mTor pathway could be probably caused by stimulation of the Igf-1 receptor by its ligand Igf 2 .

\subsubsection{Inhibitors of the PI3K/AKT/mTOR pathway}

Aberrant activation of the PI3K/AKT/mTOR pathway not only contributes to the pathogenesis of various human malignancies, it is also frequently associated with the occurrence of resistance to anticancer therapies. Therefore targeted inhibition of this pathway might possess great therapeutic potential. Actually, various PI3K/AKT/mTOR inhibitors are under investigation in a wide spectrum of cancers in clinical trials (reviewed in (Courtney et al., 2010; Lim et al., 2015). Within this work the anticancer potential of the PI3K/AKT/mTOR inhibitors PI103, GDC-0941, MK-2206, everolimus and rapamycin were investigated.

The small synthetic molecule PI103 acts as a dual PI3K/mTOR inhibitor by blocking all catalytic isoforms of PI3K. It concomitantly inhibits $\mathrm{mTOR}_{\mathrm{C} 1}$ and $\mathrm{mTOR}_{\mathrm{C} 2}$ because the catalytic subunit of PI3K and mTOR are structurally similar. Therefore dual PI3K/mTOR inhibitors are thought to completely turn off $\mathrm{PI} 3 \mathrm{~K} / \mathrm{AKT} / \mathrm{mTOR}$ signaling and prevent feedback activation usually detected with mTOR $_{\mathrm{C} 1}$ inhibitors (e.g. rapamycin, see below). However, dual PI3K/mTOR inhibitors also hold the disadvantage of greater toxicity (Courtney et al., 2010; Markman et al., 2010). PI103 inhibits cellular proliferation and invasion of various human cancer cells in vitro and shows antitumoral effects against human tumor xenografts (Fan et al., 2006; Raynaud et al., 2007). Nevertheless, due to its rapid metabolism, PI103 is not suitable for clinical application but it was seminal for the development of GDC-0941 (Raynaud et al., 2009).

GDC-0941 represents a selective and orally bioavailable inhibitor of PI3K. It was developed by optimizations of PI103 and consequently showing promising pharmacokinetic and pharmaceutical properties. In contrast to PI103, GDC-0941 possesses almost no inhibitory effect on mTOR (Raynaud et al., 2009). Nevertheless, it is effective in growth inhibition of human tumor xenografts that harbor mutations in PI3K or PTEN (Raynaud et al., 2009; Workman et al., 2010). A current phase I clinical trial that includes patients with advanced solid tumors indicates 
preliminarily evidence that GDC-0941 possess potential antitumor activity. In addition, GDC-0941 is well tolerated with frequently adverse effects such as moderate nausea, fatigue, diarrhea, and dysgeusia (reviewed in (Lim et al., 2015; Markman et al., 2010)).

MK-2206 is an orally applied allosteric inhibitor of AKT with antitumoral effects in several preclinical investigations e.g. in colon cancer and nasopharyngeal cancer cells in vitro and in vivo (Agarwal et al., 2014; Zhao et al., 2014). MK-2206 furthermore synergizes with cytotoxic drugs or other targeted therapies in preclinical studies (Hirai et al., 2010). Clinical trials using MK-2206 furthermore resulted in promising outcomes with robust abrogation of AKT signaling (Yap et al., 2011). On the basis of these positive results, several phase II studies of MK-2206 in advanced ovarian and breast cancers are currently in progress (Lim et al., 2015).

The most extensively investigated drugs targeting the PI3K/AKT/mTOR pathway are mTOR inhibitors (e.g. rapamycin, everolimus). They demonstrate antitumoral activity in haematological malignancies and solid tumors refractory to standard chemotherapies (reviewed in (Lim et al., 2015)). Rapamycin (sirolimus) is an antibiotic originally derived from Streptomyces hygroscopicus from the island of Rapa Nui. It binds to FKBP12 to form a complex that interacts with the FKBP12-rapamycin-binding domain of $\mathrm{mTOR}$ in the $\mathrm{mTOR}_{\mathrm{C} 1}$ resulting in inhibition of downstream signaling (Guertin and Sabatini, 2009). Nevertheless, some cellular models exist in which rapamycin also disrupts mTOR $_{\mathrm{C} 2}$ (Sarbassov et al., 2006). Because rapamycin showed antiproliferative effects when used as an immunosuppressive agent in transplant medicine, it was also tested for potential antitumoral properties (Law, 2005; Vemulapalli et al., 2011). However, the oral bioavailability and chemical stability of rapamycin is low and limits its clinical development as an anticancer agent (Wan and Helman, 2007). Nevertheless, synthetic derivatives of rapamycin (rapalogs) with improved pharmaceutical properties have been developed. One of the main rapalogs represents everolimus (RAD001) which is currently in clinical development (reviewed in (Lim et al., 2015)). It is orally bioavailable and shows impressive antiproliferative effects in several human tumor cell lines and human tumor xenografts (reviewed in (Fasolo and Sessa, 2012)). Furthermore, it provides encouraging outcomes in multiple phase II studies, e.g. in patients with relapsed gastric (Ohtsu et al., 2013) and with recurrent endometrial cancer (Ray-Coquard et al., 2013). Moreover, everolimus is also approved for treatment of advanced RCC after progression with sunitinib or sorafenib.

However, the efficacy of rapamycin and everolimus may be partially limited because they do not inhibit mTOR $_{\mathrm{C} 2}$ mediated phosphorylation of AKT. In addition, blocking of $\mathrm{mTOR}_{\mathrm{C} 1}$ releases the negative feedback by the S6K/IRS/PI3K cascade, which can result in a paradoxically 
increase of AKT activity (O'Reilly et al., 2006). Indeed, increased phosphorylated AKT has been observed in tumors from patients treated with everolimus (O'Reilly et al., 2006).

\subsection{Interaction of HH and PI3K/AKT/mTOR signaling}

Recently it has been assumed that different tumor related signaling pathways (e. g. $\mathrm{PI} 3 \mathrm{~K} / \mathrm{AKT} / \mathrm{mTOR}$ signaling) interact with $\mathrm{HH}$ signaling by influencing the activity of GLI transcription factors (Aberger et al., 2012). Indeed, several preclinical studies provide evidence for interactions between the HH pathway and PI3K/AKT/mTOR signaling cascade at several levels. Figure 4 shows a schematic illustration of the hitherto known crosstalk of both pathways. Since one focus of this work was on the evaluation of combination therapies using SMO antagonists plus PI3K/AKT/mTOR inhibitors in RMS, the figure also includes the respective inhibitors used in this study.

So far, several studies verified the crosstalk of HH pathway and PI3K/AKT/mTOR signaling on molecular level and showed that each pathway can influence the respective other. These studies showed on the one hand that PI3K/AKT/mTOR signaling can stimulate the activity of the $\mathrm{HH}$ pathway. For instance, Akt induces noncanonical activation of $\mathrm{HH}$ signaling by stabilization of the Gli transcriptions factors whereas inhibition of PI3K signaling inhibits Shh induced $\mathrm{HH}$ signaling (Riobo et al., 2006). Furthermore, stimulation of PI3K/Akt/mTor signaling by Igf1 can synergize with HH ligands to potentiate HH pathway activation (Rao et al., 2004; Riobo et al., 2006). Beyond that, mTOR/S6K1 mediates a SMO independent modulation of GLI activity in EAC which results in enhanced GLI transcriptional activity and oncogenic function through phosphorylation of GLI1. This result was further strengthened by a combined inhibition of the canonical and the noncanonical HH pathway using GDC-0449 and everolimus which led to an improved efficacy of tumor growth inhibition in EAC xenografts in comparison to the single treatments (Wang et al., 2012). Moreover, it has been shown that the PI3K/AKT pathway cooperates with $\mathrm{HH}$ signaling to strengthen proliferation and survival of EAC cells in vitro (Wei and $\mathrm{Xu}, 2011$ ). Another study reported a crosstalk between $\mathrm{HH}$ and PI3K/AKT pathway in tamoxifen resistant breast cancer cells, in which PI3K/AKT signaling protected GLI1 from proteasomal degradation (Ramaswamy et al., 2012). It also has been demonstrated that GLII expression and transcriptional activity is regulated through osteopontin mediated induction of AKT/GSK3b signaling (Das et al., 2013). Finally, dominant active AKT can enhance the nuclear localization of GLI1, while AKT inhibition results in cytoplasmic accumulation of GLI1 in a metastatic melanoma cell line (Stecca et al., 2007). 


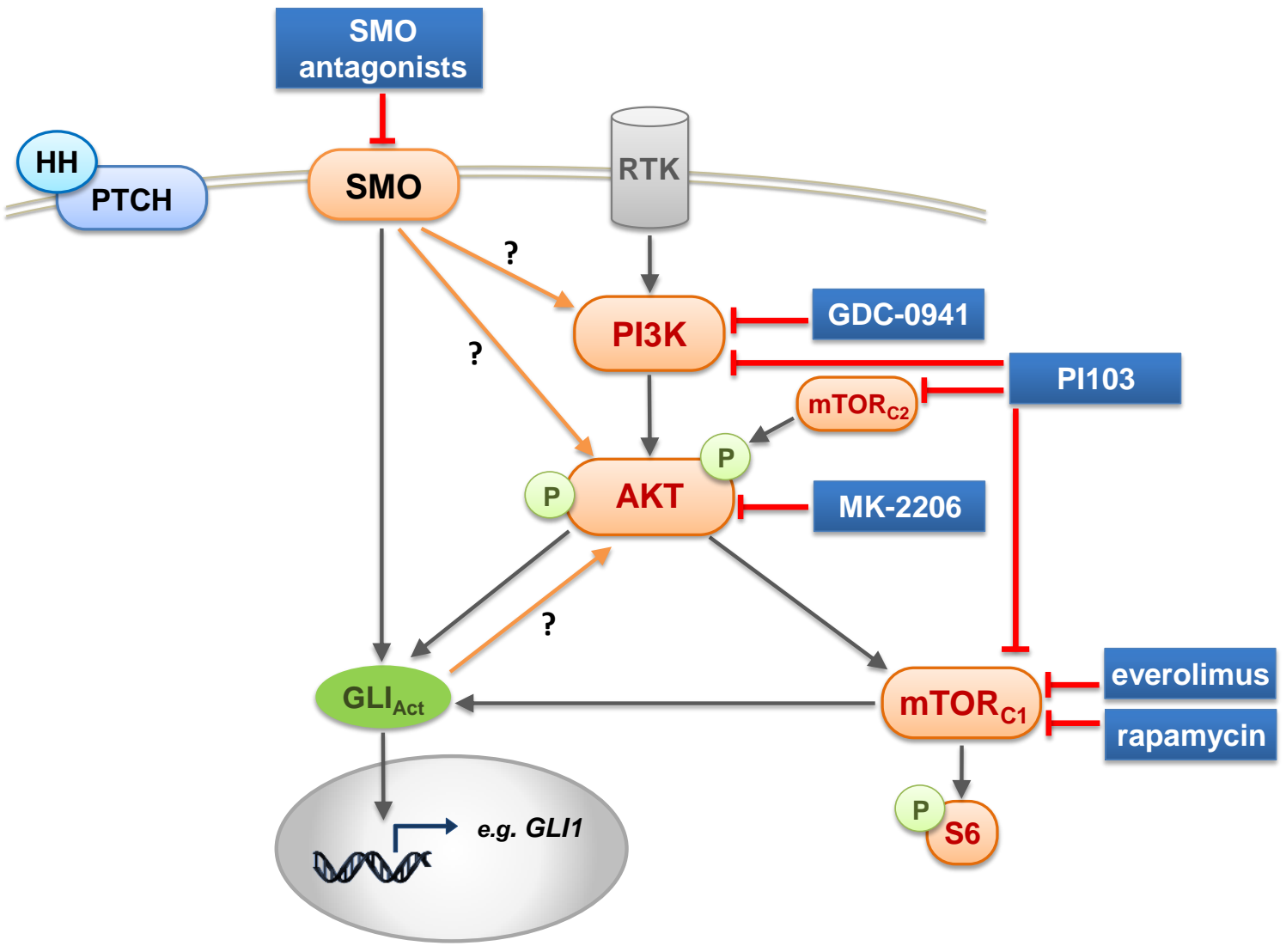

Figure 4: Interaction of HH and PI3K/AKT/mTOR signaling and associated inhibitors. The left side of the picture illustrates the $\mathrm{HH}$ signaling pathway and the right side shows a simplified scheme of the PI3K/AKT/mTOR pathway. Crosstalk of both pathways is reported due to activation and stabilization of GLI transcriptions factors by AKT and mTOR, which results in noncanonical activation of $\mathrm{HH}$ signaling. In addition, it has been shown that vice versa active HH signaling can result in activation of PI3K/AKT signaling by an unknown mechanism (illustrated by arrows with “?”). Inhibitors used in this thesis are represented in blue boxes and corresponding pharmacological targets at several points along the pathway are indicated in red $\dashv$.

On the other hand, $\mathrm{HH}$ signaling can stimulate the activity of the PI3K/AKT/mTOR pathway, e.g. in bone marrow derived endothelial progenitor cells and pancreatic duct epithelial cells active Hh signaling can result in activation of PI3K/Akt (Fu et al., 2006; Morton et al., 2007). Similarly, SHH treatment activated AKT in human gastric cancer cells and conversely PI3K/AKT pathway inhibition blocked SHH induced epithelial mesenchyme transition (EMT) and reduced tumor invasiveness and metastasis (Yoo et al., 2011).

Finally, also in RMS the two pathways seem to interact since the inhibition of mTOR by rapamycin blocks Hh target gene transcription (measured by Gli1, Gli2 and Ptch) and reduces Gli1 and Ptch protein levels, leading to inhibition of RMS growth in vivo (Kaylani et al., 2013). 
All these findings indicate that the $\mathrm{HH}$ and $\mathrm{PI} 3 \mathrm{~K} / \mathrm{AKT} / \mathrm{mTOR}$ signaling pathways can regulate each other in a positive feedback loop and can cooperate in tumor formation. The crosstalk of both pathways provides rationales for combining SMO antagonists with inhibitors of the $\mathrm{PI} 3 \mathrm{~K} / \mathrm{AKT} / \mathrm{mTOR}$ pathway to synergistically block both oncogenic pathways and to improve and optimize tumor therapies. Indeed, several clinical trials are in process, e.g. the treatment of advanced solid tumors (including pancreatic cancer) or recurrent glioblastoma, CRC, breast and pancreatic cancer with GDC-0449 plus sirolimus or LDE225 plus the PI3K inhibitor BKM120, respectively (Amakye et al., 2013). 


\section{AIM OF THE STUDY}

The aim of this thesis was to evaluate novel therapeutic strategies for RMS treatment. The HH and the PI3K/AKT/mTOR pathways play an important role in many cancers including RMS.

First, the HH signaling pathway was targeted by SMO inhibitors in order to analyze if they might be beneficial for RMS treatment. Hence, potential antitumoral effects of GDC-0449, LDE225, HhA and cyclopamine were compared in four human RMS cell lines, i.e. two ERMS (RD, RUCH-2) and two ARMS (RMS-13, Rh41) cell lines. For this purpose the effects on HH signaling activity (measured by GLII expression), on IGF2 expression and on the differentiation status were investigated. Moreover, effects on cellular proliferation and on apoptosis as well as the impact on the PI3K/AKT/mTOR pathway, on AMPK activity and on autophagy were analyzed.

Second, in order to enhance possible anticancer effects and to test for potential cooperative effects, SMO antagonists were combined with PI3K/AKT/mTOR pathway inhibitors. The latter included the dual PI3K/mTOR inhibitor PI103, the specific PI3K inhibitor GDC-0941, the pure AKT inhibitor MK-2206 and the mTOR inhibitors everolimus and rapamycin. Initial experiments indicated that $\mathrm{HhA}$ was the most promising of the four SMO antagonists for combination treatment. Therefore, HhA was used for further experiments in order to find a valuable drug combination. Finally, since HhA plus GDC-0941 showed cooperative anticancer effects in all four RMS cell lines, this combination was evaluated in vivo using RMS bearing $\mathrm{Ptch}^{+/-}$mice. 


\section{MATERIAL}

\subsection{Technical equipment}

Table 1: List of laboratory equipment

\begin{tabular}{|c|c|}
\hline Equipment & Supplier \\
\hline$-20^{\circ} \mathrm{C}$ Freezer & Liebherr GmbH, Ochshausen \\
\hline$-80^{\circ} \mathrm{C}$ Freezer (MDF-U71V) & Sanyo Electric Co., Ltd., Japan \\
\hline $4{ }^{\circ} \mathrm{C}$ Fridge & Robert Bosch GmbH, Stuttgart \\
\hline Agarose gel electrophoresis chamber & Peqlab Biotechnology GmbH, Erlangen \\
\hline Autoclave (Systec DX-150) & Systec GmbH \& Co. KG, Linden \\
\hline Biophotometer (6131) & Eppendorf AG, Hamburg \\
\hline Centrifuges (Biofuge fresco, primo) & Kendro Laboratory Products GmbH, Hanau \\
\hline Cold Plate (EG 1150 C) & Leica Microsystems GmbH, Wetzlar \\
\hline Digital monochrome printer (P91D) & Mitsubishi, Ratingen \\
\hline Digital photo camera (PowerShot G2) & Canon Deutschland GmbH, Krefeld \\
\hline Dissecting set & Karl Hammacher GmbH, Solingen \\
\hline Electronic pipettor (Accu-jet) & Brand GmbH \& Co. KG, Wertheim \\
\hline $\begin{array}{l}\text { Electrophoresis System (XCell4 SureLock }{ }^{\mathrm{TM}} \\
\text { Midi-Cell) }\end{array}$ & Invitrogen $\mathrm{GmbH}$, Karlsruhe \\
\hline FACS Calibur & BD Biosciences GmbH, Heidelberg \\
\hline Freezing Container (Mr. Frosty ${ }^{\mathrm{TM}}$ ) & Thermo Fisher Scientific GmbH, Schwerte \\
\hline Heating block (Thermomixer) & Eppendorf AG, Hamburg \\
\hline Heating stirrer (MR 3000/3001) & $\begin{array}{l}\text { Heidolph Instruments GmbH \& Co. KG, } \\
\text { Schwabach }\end{array}$ \\
\hline High-precision scales (Sartorius Basic plus) & Sartorius AG, Göttingen \\
\hline Hybridization oven (HB-1000 Hybridizer) & UVP, Inc., Upland, USA \\
\hline Imaging system Fluorchem Q & Fisher Scientific GmbH, Schwerte \\
\hline Incubator (6000, BBD 6220) & Kendro Laboratory Products GmbH, Hanau \\
\hline $\begin{array}{l}\text { Inverted tissue culture fluorescence microscope } \\
\text { (Axiovert 25) }\end{array}$ & Carl Zeiss Jena GmbH, Jena \\
\hline $\begin{array}{l}\text { Laboratory animal computed tomography system } \\
\text { (QuantumFX) }\end{array}$ & PerkinElmer Health Sciences, Hopkinton USA \\
\hline Liquid nitrogen tank & L'air liquid S.A., Paris, France \\
\hline Mastercycler (EP gradient S) & Eppendorf AG, Hamburg \\
\hline Microplate reader (Synergy Mx) & BioTek Instruments, Inc., Bad Friedrichshall \\
\hline
\end{tabular}




\begin{tabular}{ll}
\hline Equipment & Supplier \\
\hline Microscope (Olympus BX 60) & Olympus Deutschland GmbH, Hamburg \\
Microtom (HN 40) & Leica Microsystems GmbH, Wetzlar \\
Microwave (Dimension 4) & Panasonic, Hamburg \\
Mini centrifuge & Carl Roth GmbH \& Co. KG, Karlsruhe \\
Multifuge (Heraeus 3LR) & Thermo Scientific, Wilmington, USA \\
Orbital shaker (Unimax 1010) & Heidolph Instruments GmbH \& Co. KG, \\
Paraffin dispenser (Dispenser PAG 12) & Schwabach \\
Paraffin tissue floating bath & Medite GmbH, Burgdorf \\
PCR machine & Medax GmbH \& Co. KG, Rendburg \\
pH-meter (inoLab pH Level 1) & Eppendorf, Hamburg \\
Pipettes (Multipette, One-channel) & WTW GmbH, Vienna, Austria \\
Power supply for electrophoresis & Eppendorf AG, Hamburg \\
Real-Time PCR System (ABI Prism 7900HT) & Peqlab Biotechnology GmbH, Erlangen \\
Spectrophotometer (NanoDrop 8000 ) & Thermo Scientific, Wilmington, USA \\
Stereo microscope (Stemi 2000) & Carl Zeiss Jena GmbH, Jena \\
Sterile bench (Euroflow Class IIA) & Clean Air Techniek bv, Woerden, Netherlands \\
Tissue embedding and rehydrating machine (TP & Leica Microsystems GmbH, Wetzlar \\
1020) & Bio-Rad Laboratories GmbH, Munich \\
Trans-Blot SD semi-dry transfer cell & Intas Science Imaging GmbH, Göttingen \\
Vacuum pump & Schütt Labortechnik, Göttingen \\
Vortexer-Genie 2 & Scientific Industries, Woburn, USA \\
Water purification system (Arium® 611 VF) & Sartorius, Göttingen \\
\hline & \\
\hline
\end{tabular}




\subsection{Consumables}

Table 2: List of consumable materials

\begin{tabular}{|c|c|}
\hline Consumer good & Supplier \\
\hline $1.5 \mathrm{ml}$ reaction tubes & Ochs GmbH, Bovenden/Lenglern \\
\hline $1.5 \mathrm{ml}$ safeseal microtubes & Sarstedt AG \& Co., Nürnberg \\
\hline $2.0 \mathrm{ml}$ reaction tubes & Sarstedt AG \& Co., Nürnberg \\
\hline 6-well tissue culture plate & Sarstedt AG \& Co., Nürnberg \\
\hline $15 \mathrm{ml}$ centrifuge tubes & Greiner Bio-One GmbH, Frickenhausen \\
\hline $50 \mathrm{ml}$ centrifuge tubes & Greiner Bio-One GmbH, Frickenhausen \\
\hline 96-well assay plate & Nunc GmbH \& Co. KG, Wiesbaden \\
\hline 96-well reaction plate (black) & Costar Corning Incorporated, Corning, USA \\
\hline 96-well PCR plate (non-skirted) & 4titude Ltd., Berlin \\
\hline 384-well PCR plate (Framestar) & 4titude Ltd., Berlin \\
\hline BD Discardit $^{\mathrm{TM}}$ II $(2,10,20 \mathrm{ml})$ & BD Biosciences GmbH, Heidelberg \\
\hline BD Microfine + Demi & BD Biosciences GmbH, Heidelberg \\
\hline BD Plastipak & BD Biosciences GmbH, Heidelberg \\
\hline Blotting paper (GB 33 B003) & Heinemann Labortechnik GmbH, Duderstadt \\
\hline Cell culture dishes (Nunclon Surface) & Nunc GmbH \& Co.KG, Wiesbaden \\
\hline Cell scraper & Sarstedt AG \& Co., Nürnberg \\
\hline Cell strainers $(40 \mu \mathrm{m})$ & BD Biosciences GmbH, Heidelberg \\
\hline Combitips $(0.2,0.5,2.5,5,10,25,50 \mathrm{ml})$ & Eppendorf AG, Hamburg \\
\hline Coverslips & Menzel GmbH \& Co.KG, Braunschweig \\
\hline CryoPure tubes & Sarstedt AG \& Co., Nürnberg \\
\hline Disposable needles (Sterican $\varnothing 0,45$ x $12 \mathrm{~mm}$ ) & B. Braun Medical AG, Emmenbrücke \\
\hline Feeding tubes $(1.0 \times 60 \mathrm{~mm})$ & Unimed SA, Lausanne, Schweiz \\
\hline Filter tips $(10 \mu \mathrm{l})$ & Sarstedt AG \& Co., Nürnberg \\
\hline Filter tips $(100 \mu \mathrm{l}, 200 \mu \mathrm{l}, 1000 \mu \mathrm{l})$ & Kisker Biotech GmbH \& Co. KG, Steinfurt \\
\hline Flow cytometry tube & Sarstedt AG \& Co., Nürnberg \\
\hline Fluted filters & Sartorius AG, Göttingen \\
\hline Glassware & Schott AG, Mainz \\
\hline Milliporefilter (Nuclepore Track-Etch Membran) & Whatman GmbH, Dassel \\
\hline Miscroscope slides (SuperFrost Plus) & Menzel GmbH \& Co.KG, Braunschweig \\
\hline Neubauer counting chamber & Brand GmbH \& Co KG, Wertheim \\
\hline Nitrocellulose membrane (Hybond ECL) & GE Healthcare Europe GmbH, Freiburg \\
\hline
\end{tabular}




\begin{tabular}{ll}
\hline Consumer good & Supplier \\
\hline NuPAGE Novex $4-12 \%$ Bis-Tris Midi Gel & Invitrogen GmbH, Karlsruhe \\
Pasteur pipettes & Brand GmbH \& Co.KG, Wertheim \\
Petri dishes & Ochs GmbH, Bovenden/Lenglern \\
Pipette tips $(10 \mu l, 200 \mu \mathrm{l})$ & Ochs GmbH, Bovenden/Lenglern \\
Pipette tips $(20 \mu 1,1000 \mu \mathrm{l})$ & Sarstedt AG \& Co., Nürnberg \\
QPCR adhesive clear seal & 4titude Ltd., Berlin \\
Scalpel blade $(10,24)$ & Aesculap AG \& Co.KG, Tuttlingen \\
Serological pipettes $(2 \mathrm{ml}, 5 \mathrm{ml}, 10 \mathrm{ml}, 25 \mathrm{ml}$, & Sarstedt AG \& Co., Nürnberg \\
$50 \mathrm{ml})$ & Omnilab-Krannich, Göttingen \\
Sterile filter & Terumo Medical Corp., Elkton, MD, USA \\
Syringe $30,50 \mathrm{ml}$ & \\
\hline
\end{tabular}

\subsection{Reagents and chemicals}

Chemicals which are not listed below were purchased from Sigma-Aldrich Chemie GmbH, Steinheim.

Table 3: Utilized reagents and chemicals

\begin{tabular}{ll}
\hline Chemicals and reagents & Supplier \\
\hline 50 bp, 100 bp plus, 1 kb DNA Ladder & Fermentas GmbH, St. Leon-Rot \\
Acetic acid & Carl Roth GmbH \& Co. KG, Karlsruhe \\
Agarose & Bio-Budget Technologies GmbH, Krefeld \\
Ampuwa & Fresenius Kabi Deutschland GmbH, Bad \\
AnnexinV-FITC & Homburg \\
Boric acid & BD Biosciences GmbH, Heidelberg \\
Bovine serum albumin (BSA) & Carl Roth GmbH \& Co. KG, Karlsruhe \\
Chloroform & Carl Roth GmbH \& Co. KG, Karlsruhe \\
Citric acid & Carl Roth GmbH \& Co. KG, Karlsruhe \\
Collagenase H & Carl Roth GmbH \& Co. KG, Karlsruhe \\
Contrast agent Imeron 300 & Roche Diagnostics GmbH, Mannheim \\
Deoxyribonucleotide triphosphate (dNTP) & Bracco Imaging GmbH, Konstanz \\
Dithiothreitol, 100mM (DTT) & Roche Diagnostics GmbH, Mannheim
\end{tabular}




\begin{tabular}{|c|c|}
\hline Chemicals and reagents & Supplier \\
\hline DNase/RNase-free distilled water & GIBCO Invitrogen $\mathrm{GmbH}$, Karlsruhe \\
\hline Doxorubicin & $\begin{array}{l}\text { Pharmacy of the university medical center, } \\
\text { Göttingen }\end{array}$ \\
\hline Eosin $\mathrm{Y}$ & Merck KGaA, Darmstadt \\
\hline Ethanol (EtOH) $99 \%$ & J.T. Baker B.V., Deventer, Netherlands \\
\hline Ethidium bromide $(0.07 \%)$ & inna-TRAIN-Diagnostics, Kronberg \\
\hline Ethylenediaminetetraacetic acid (EDTA) & ICN Biochemicals Inc., Aurora, USA \\
\hline EtOH $99 \%$ denatured & $\begin{array}{l}\text { CVH Chemie-Vertrieb GmbH \& Co. } \\
\text { Hannover KG, Hannover }\end{array}$ \\
\hline Glycergel mounting medium & Dako GmbH, Hamburg \\
\hline Haematoxylin, Mayer's & Merck KGaA, Darmstadt \\
\hline Hydrogen peroxide & Carl Roth GmbH \& Co. KG, Karlsruhe \\
\hline Isoflurane $\left(\mathrm{FORENE}^{\circledR}\right)$ & Abbott GmbH \& Co. KG, Wiesbaden \\
\hline Isopropyl alcohol & Carl Roth GmbH \& Co. KG, Karlsruhe \\
\hline Methanol & Carl Roth GmbH \& Co. KG, Karlsruhe \\
\hline NuPAGE MES SDS Running Buffer, $20 \mathrm{x}$ & Invitrogen $\mathrm{GmbH}$, Karlsruhe \\
\hline Paraformaldehyde & Carl Roth GmbH \& Co. KG, Karlsruhe \\
\hline Pertex mounting medium & Medite Medizintechnik GmbH, Burgdorf \\
\hline Phosphatase inhibitor cocktail tablets (PhosSTOP) & Roche Diagnostics GmbH, Mannheim \\
\hline Phosphate buffered saline (PBS)-Tablets & GIBCO Invitrogen $\mathrm{GmbH}$, Karlsruhe \\
\hline Potassium aluminum sulfate & Merck KGaA, Darmstadt \\
\hline Powdered milk & Carl Roth GmbH \& Co. KG, Karlsruhe \\
\hline Propidium Iodide (PI) & Miltenyi Biotec, Bergisch Gladbach \\
\hline Protease inhibitor cocktail tablets (Complete, mini) & Roche Diagnostics GmbH, Mannheim \\
\hline Proteinase $\mathrm{K}$ & Carl Roth GmbH \& Co. KG, Karlsruhe \\
\hline Random Hexamer-Oligonucleotides & Invitrogen $\mathrm{GmbH}$, Karlsruhe \\
\hline SeeBlue ${ }^{\circledR}$ Plus2 Pre-Stained Standard & Invitrogen $\mathrm{GmbH}$, Karlsruhe \\
\hline Sodiumdodecylsulfate (SDS) & Carl Roth GmbH \& Co. KG, Karlsruhe \\
\hline Trichloro acetaldehyde hydrate & Merck KGaA, Darmstadt \\
\hline TRIzol Reagent & Invitrogen $\mathrm{GmbH}$, Karlsruhe \\
\hline Water soluble tetrazolium salt-1 (WST-1) reagent & Roche Diagnostics GmbH, Mannheim \\
\hline Xylene & J.T. Baker B.V., Deventer, Netherlands \\
\hline
\end{tabular}




\subsection{Signaling pathway inhibitors}

Drugs, their respective solvents and final concentrations for in vitro analyses are listed in Table 4.

Table 4: List of applied inhibitors

\begin{tabular}{|c|c|c|c|}
\hline Inhibitor & Solvent & $\begin{array}{l}\text { Incubation } \\
\text { concentration }\end{array}$ & Supplier \\
\hline Cyclopamine & $\mathrm{EtOH}$ & $0.1-10 \mu \mathrm{M}$ & Sigma-Aldrich, Steinheim \\
\hline Everolimus & $\mathrm{EtOH}$ & $50 \mathrm{nM}$ & Sigma-Aldrich, Steinheim \\
\hline $\begin{array}{l}\text { GDC-0449 } \\
\text { (Vismodegib) }\end{array}$ & DMSO & $0.1-50 \mu \mathrm{M}$ & $\begin{array}{l}\text { Selleckchem, Munich (in vitro); } \\
\text { Genentech, San Francisco, USA (in } \\
\text { vivo) }\end{array}$ \\
\hline GDC-0941 & DMSO & $10 \mu \mathrm{M}(500 \mathrm{nM}$ in $\mathrm{Rh} 41)$ & Genentech, San Francisco, USA \\
\hline $\begin{array}{l}\text { HhA } \\
\text { (HhAntag691) }\end{array}$ & DMSO & $0.1-50 \mu \mathrm{M}$ & Genentech, San Francisco, USA \\
\hline $\begin{array}{l}\text { LDE225 } \\
\text { (NVP-LDE225, } \\
\text { Sonidegib) }\end{array}$ & DMSO & $0.1-50 \mu \mathrm{M}$ & Active Biochem, Bonn \\
\hline MK-2206 & DMSO & $\begin{array}{l}5 \mu \mathrm{M}(1 \mu \mathrm{M} \text { in RMS-13 } \\
\text { and Rh41) }\end{array}$ & Selleckchem, Munich \\
\hline PI103 & DMSO & $3 \mu \mathrm{M}(200 \mathrm{nM}$ in $\mathrm{Rh} 41)$ & Axxora Deutschland GmbH, Lörrach \\
\hline Rapamycin & DMSO & $100 \mathrm{nM}$ & Calbiochem, Merck KGaA, Darmstad \\
\hline SAG (SMO agonist) & DMSO & $10 \mathrm{nM}-1 \mu \mathrm{M}$ & Cayman chemicals, Ann Arbor USA \\
\hline
\end{tabular}


MATERIAL

\subsection{Kits and ready-to-use reaction systems}

Unless indicated otherwise, all kits and ready-to-use reaction systems were used according to the manufacturer's instructions.

Table 5: Kits and ready-to-use reaction systems

\begin{tabular}{|c|c|}
\hline Reaction system & Supplier \\
\hline $5 \times 1$ st strand buffer & Invitrogen $\mathrm{GmbH}$, Karlsruhe \\
\hline $10 \mathrm{x}$ AnnexinV binding buffer & BD Biosciences GmbH, Heidelberg \\
\hline $\begin{array}{l}\text { Cell Proliferation ELISA, BrdU } \\
\text { (chemiluminescent) }\end{array}$ & Roche Diagnostics GmbH, Mannheim \\
\hline Dako EnVision $^{\mathrm{TM}}$ detection System & Dako Denmark A/S, Glostrup, Denmark \\
\hline Pierce BCA Protein Assay Kit & Fisher Scientific GmbH, Schwerte \\
\hline Pierce ECL western blot substrate & Fisher Scientific GmbH, Schwerte \\
\hline Platinum SYBR Green qPCR SuperMix & Invitrogen $\mathrm{GmbH}$, Karlsruhe \\
\hline Reverse Transcriptase (SuperScriptII ${ }^{\circledR}$ ) & Invitrogen $\mathrm{GmbH}$, Karlsruhe \\
\hline Taq-Polymerase (MolTaq) & Molzym GmbH \& Co. KG, Bremen \\
\hline
\end{tabular}

\subsection{Buffers and solutions}

Unless mentioned otherwise, all solutions were prepared with double distilled water $\left(\mathrm{ddH}_{2} \mathrm{O}\right)$.

Table 6: Buffers and solutions and their respective composition

\begin{tabular}{ll}
\hline Buffer & Composition \\
\hline 6 x SDS loading buffer & $35 \%(\mathrm{v} / \mathrm{v})$ Glycerol \\
& $9 \%(\mathrm{w} / \mathrm{v}) \mathrm{SDS}$ \\
& $8.5 \%(\mathrm{w} / \mathrm{v}) \mathrm{DTT}$ \\
& $0.1 \%(\mathrm{w} / \mathrm{v})$ Bromphenolblue \\
& dissolved in Upper gel buffer \\
\hline 10 x PBS, pH 7.4 & $1.4 \mathrm{M} \mathrm{NaCl}$ \\
& $65 \mathrm{mM} \mathrm{Na}_{2} \mathrm{HPO}_{4}$ \\
& $27 \mathrm{mM} \mathrm{KCl} \mathrm{mM} \mathrm{KH}_{2} \mathrm{PO}_{4}$ \\
\hline
\end{tabular}




\begin{tabular}{|c|c|}
\hline Buffer & Composition \\
\hline \multirow{3}{*}{$\begin{array}{l}10 \times \text { Tris-boric acid-EDTA (TBE), } \\
\text { pH } 8.0\end{array}$} & 890 mM Tris/HCl \\
\hline & $730 \mathrm{mM}$ Boric acid \\
\hline & $12.5 \mathrm{mM}$ EDTA \\
\hline \multirow{2}{*}{$\begin{array}{l}10 \times \text { Tris-buffered saline (TBS), } \\
\text { pH } 7.4\end{array}$} & $1.5 \mathrm{M} \mathrm{NaCl}$ \\
\hline & $0.5 \mathrm{M}$ Tris/HCl \\
\hline \multirow{3}{*}{ AEC chromogen, pH 5.2} & $70 \mathrm{mM}$ Sodium acetate trihydrate \\
\hline & $30 \mathrm{mM}$ Acetic acid \\
\hline & $\begin{array}{l}16 \mathrm{mM} \text { 3-Amino-9 Ethylcarbazole (dissolved } \\
\text { in dimethylformamide) }\end{array}$ \\
\hline \multirow[t]{4}{*}{ Blotting buffer } & $20 \%(\mathrm{v} / \mathrm{v})$ Methanol \\
\hline & $6 \%(\mathrm{w} / \mathrm{v})$ Tris \\
\hline & $3 \%(w / v)$ Glycine \\
\hline & $0,0375 \%(\mathrm{v} / \mathrm{v}) \mathrm{SDS}$ \\
\hline \multirow[t]{3}{*}{ BSA-azide } & $0.02 \%$ Sodium azide \\
\hline & $2 \% \mathrm{BSA}$ \\
\hline & dissolved in PBST \\
\hline Citric acid buffer, $\mathrm{pH} 6.0$ & 10 mM Sodium Citrate \\
\hline \multirow{2}{*}{ Collagen solution } & $0.1 \mathrm{M}$ Acetic acid \\
\hline & $1 \mathrm{mg} / \mathrm{ml}$ Collagen \\
\hline \multirow[t]{2}{*}{ Cresol } & $0.1 \%(\mathrm{w} / \mathrm{v})$ Cresol \\
\hline & saturated sucrose solution \\
\hline \multirow[t]{4}{*}{ dNTP-Mix } & $10 \mathrm{mM}$ dATP \\
\hline & $10 \mathrm{mM}$ dCTP \\
\hline & $10 \mathrm{mM}$ dGTP \\
\hline & $10 \mathrm{mM}$ dTTP \\
\hline \multirow[t]{2}{*}{ Eosin solution } & $80 \%(\mathrm{v} / \mathrm{v}) \mathrm{EtOH}$ \\
\hline & $1 \%(w / v)$ Eosin y (water soluble) \\
\hline \multirow[t]{5}{*}{ Haematoxylin solution, Mayer's } & $5 \%(\mathrm{w} / \mathrm{v})$ Potassium aluminum sulfate \\
\hline & $5 \%(\mathrm{w} / \mathrm{v})$ Trichloro acetaldehyde hydrate \\
\hline & $1 \%(\mathrm{w} / \mathrm{v})$ Citric acid \\
\hline & $0.1 \%(w / v)$ Haematoxylin \\
\hline & $0.015 \%(\mathrm{w} / \mathrm{v})$ Sodium iodate \\
\hline
\end{tabular}




\begin{tabular}{|c|c|}
\hline Buffer & Composition \\
\hline \multirow[t]{7}{*}{ Lysis buffer, $\mathrm{pH} 8.8$} & $150 \mathrm{mM} \mathrm{NaCl}$ \\
\hline & $30 \mathrm{mM}$ Tris/HCl \\
\hline & $10 \%(\mathrm{v} / \mathrm{v})$ Glycerol \\
\hline & $1 \%(\mathrm{v} / \mathrm{v})$ Triton $\mathrm{X}-100$ \\
\hline & Protease and phosphatase inhibitors ( 1 tablet $/ 50 \mathrm{ml})$ \\
\hline & 2 mM DTT (added directly before use) \\
\hline & $\begin{array}{l}500 \mu \mathrm{M} \text { Phenylmethanesulfonylfluoride (PMSF) (added } \\
\text { directly before use) }\end{array}$ \\
\hline \multirow{2}{*}{ Methyl cellulose tween (MCT) } & $0.5 \%$ (w/v) Methylcellulose \\
\hline & $0.2 \%(\mathrm{w} / \mathrm{v})$ Tween -80 \\
\hline Paraformaldehyde (PFA) & $4 \%(w / v)$ Paraformaldehyde dissolved in PBS \\
\hline PBS (cell culture) & 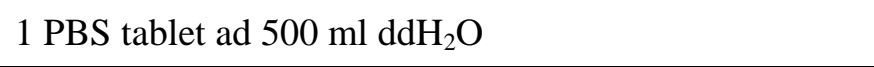 \\
\hline PBS-Tween (PBST) & $0.1 \%$ Tween-20 dissolved in PBS \\
\hline \multirow[t]{3}{*}{ Proteinase $\mathrm{K}, \mathrm{pH} 8.0$} & $50 \mathrm{mM}$ Tris/HCl \\
\hline & 5 mM EDTA \\
\hline & $10 \mathrm{mg} / \mathrm{ml}$ Proteinase $\mathrm{K}$ \\
\hline \multirow[t]{4}{*}{ STE-Buffer, pH 8.0} & $100 \mathrm{mM} \mathrm{NaCl}$ \\
\hline & $50 \mathrm{mM}$ Tris/HCl \\
\hline & $1 \mathrm{mM}$ EDTA \\
\hline & $1 \%(\mathrm{w} / \mathrm{v}) \mathrm{SDS}$ \\
\hline TBS-Triton X-100 (TBST) & $0.1 \%$ Triton $\mathrm{X}-100$ dissolved in TBS \\
\hline Trypan blue & $0.4 \%$ (w/v) Trypan blue dissolved in PBS \\
\hline \multirow[t]{2}{*}{ Upper gel buffer, pH 6.8} & $6 \%(\mathrm{w} / \mathrm{v})$ Tris \\
\hline & $0.4 \%(\mathrm{v} / \mathrm{v}) \mathrm{SDS}$ \\
\hline
\end{tabular}




\subsection{Media and reagents for cell culture}

Table 7: Media and reagents used for cell culture

\begin{tabular}{ll}
\hline Media and reagents & Supplier \\
\hline Accutase & PAA Laboratories GmbH, Pasching \\
Dulbecco's Modified Eagle Medium (DMEM) & Gibco, Invitrogen GmbH, Karlsruhe \\
Fetal calf serum (FCS) & Gibco, Invitrogen GmbH, Karlsruhe \\
Penicillin (10.000 U/ml)/Streptomycin $(10 \mathrm{mg} / \mathrm{ml})(\mathrm{P} / \mathrm{S})$ & PAN Biotech GmbH, Aidenbach \\
RPMI 1640 (RPMI) & Gibco, Invitrogen GmbH, Karlsruhe \\
TrypLE Express & Gibco, Invitrogen GmbH, Karlsruhe \\
\hline
\end{tabular}

\subsection{Cell lines and primary RMS cells}

Table 8: List of cell lines and their corresponding culture conditions

\begin{tabular}{llll}
\hline Cell line & Subtype & Medium and supplements & Supplier \\
\hline RD & human ERMS & DMEM, 10\% FCS, 1\% P/S & ATCC \\
RUCH-2 & human ERMS & DMEM, 10\% FCS, 1\% P/S & ATCC \\
RMS-13 & human ARMS & RPMI, 10\% FCS, 1\% P/S & ATCC \\
Rh41 & human ARMS & RPMI, 20\% FCS, 1\% P/S & ATCC \\
$\begin{array}{l}\text { Primary } \text { Ptch }^{+-} \text {RMS } \\
\text { cells }\end{array}$ & murine ERMS-like & DMEM, 10\% FCS, 1\% P/S & \\
\hline
\end{tabular}




\subsection{Synthetic DNA-oligonucleotides}

Synthetic DNA-oligonucleotides (primers) were obtained from Eurofins MWG Operon, Ebersberg. For long-term storage (at $-80{ }^{\circ} \mathrm{C}$ ) $100 \mu \mathrm{M}$ stock solutions in $\mathrm{ddH}_{2} \mathrm{O}$ were prepared and $10 \mu \mathrm{M}$ working solutions were applied for polymerase chain reaction (PCR) methods. PCR for genotyping of $\mathrm{Ptch}^{+-}$mice was performed using primers presented in Table 9.

Table 9: Oligonucleotides for genotyping of $\mathrm{Ptch}^{+/}$mice

\begin{tabular}{|c|c|c|c|}
\hline $\begin{array}{l}\text { Primer } \\
\text { name }\end{array}$ & $\begin{array}{l}\text { Amplification } \\
\text { product }\end{array}$ & Primer sequence ( $5^{\prime}-3^{\prime}$ orientation) & Reference \\
\hline neo-L & $\begin{array}{l}\text { Together with Pst } 4 \text { KF: } \\
634 \text { bp (wild type allele) }\end{array}$ & AGTGCCAGCGGGGCTGCTAAA & \multirow{3}{*}{$\begin{array}{l}\text { (Hahn et } \\
\text { al., 1998) }\end{array}$} \\
\hline mPTC11R3 & $\begin{array}{l}\text { Together with Pst } 4 \mathrm{KF} \text { : } \\
400 \text { bp (mutant allele) }\end{array}$ & CTGCCTGTTATGTGGTTCAAACCG & \\
\hline Pst4KF & & GGGAGGGGATTTCAGCAGAATGTT & \\
\hline
\end{tabular}

Oligonucleotides used for analysis of gene expression levels via quantitative real-time PCR (qRT-PCR) are listed in Table 10.

Table 10: Oligonucleotides for qRT-PCR

\begin{tabular}{|c|c|c|c|}
\hline Transcript & $\begin{array}{l}\text { Primer name } \\
\text { forward/reverse }\end{array}$ & $\begin{array}{l}\text { Primer sequence } \\
(5,-3 \text { ' orientation })\end{array}$ & $\begin{array}{c}\text { Primer } \\
\text { location in } \\
\text { Exon }\end{array}$ \\
\hline \multirow[t]{2}{*}{$18 S$} & $18 \mathrm{~S}$-fwd & CGCAAATTACCCACTCCCG & 1 \\
\hline & $18 \mathrm{~S}-\mathrm{rev} 2$ & TTCCAATTACAGGGCCTCGAA & 1 \\
\hline \multirow[t]{2}{*}{$h G L I 1$} & hsaGLI1 tqF & AGCTACATCAACTCCGGCCA & 11 \\
\hline & hsaGLI1 tqR & GCTGCGGCGTTCAAGAGA & 12 \\
\hline \multirow[t]{2}{*}{$h I G F 2$} & hIGF2 F2 & GACACCCTCCAGTTCGTCTG & 2 \\
\hline & hIGF2 F1 & ATTGGAAGAACTTGCCCACG & 4 \\
\hline \multirow[t]{2}{*}{$h M Y H 1$} & hsMYH1F.1 & TGTGCAGCAGGTGTACAATGC & 13,14 \\
\hline & hsMYH1R.1 & TGCACAGCTGCTCCAGGCT & 15 \\
\hline \multirow[t]{2}{*}{ hMYOD } & hMYOD F & CGAACCCCCAACCCGATA & 3 \\
\hline & hMYOD R & GAAAAAACCGCGCTGTGT & 3 \\
\hline
\end{tabular}




\subsection{Antibodies}

Table 11: Antibodies for western blot and immunohistochemistry

\begin{tabular}{lcll}
\hline Antibody & Dilution & Source & Supplier \\
\hline Primary antibody & & & \\
Anti-AKT; (610861) & $1: 1000$ & Mouse, mAB & BD Biosciences \\
Anti-AMPK & $1: 1000$ & Rabbit, pAB & Cell Signaling \\
Anti-B-Actin; (13E5) & $1: 1000$ & Rabbit, mAB & Cell Signaling \\
Anti-Caspase 3 & $1: 1000$ & Rabbit, pAB & Cell Signaling \\
Anti-HSC70; (sc-7298) & $1: 10000$ & Mouse, mAB & Santa Cruz \\
Anti-IGF2; (LS-C165143) & $1: 500$ & Rabbit, pAB & LSBio \\
Anti-Ki67 & $1: 50$ & Mouse, mAB & BD Biosciences \\
Anti-LC3-II; (D11 XP) & $1: 1000$ & Rabbit, mAB & Cell Signaling \\
Anti-pAKT (Ser473); (193H12) & $1: 1000$ & Rabbit, mAB & Cell Signaling \\
Anti-pAMPK (Thr172); (40H9) & $1: 1000$ & Rabbit, mAB & Cell Signaling \\
Anti-pS6 (Ser240/244) & $1: 1000$ & Rabbit, pAB & Cell Signaling \\
Anti-S6; (54D2) & $1: 1000$ & Mouse, mAB & Cell Signaling
\end{tabular}

\section{Secondary antibody}

Anti-Mouse/HRP; (NA931)

1:5000 Sheep, pAB

GE Healthcare

Anti-Rabbit/HRP; (A0545)

1:5000 Goat, pAB

Sigma-Aldrich

Dako Envision/HRP

undiluted

Dako

mAB: monoclonal antibody, pAB: polyclonal antibody, HSC70: heat-shock protein 70, HRP: horseradish peroxidase conjugated 
MATERIAL

\subsection{Software}

Table 12: List of used software

\begin{tabular}{ll}
\hline Software & Developer \\
\hline Adobe Photoshop CS5 & Adobe Systems Incorporated, San Jose, USA \\
AlphaView Q SA 3.2.2 & Cell Bioscience, California, USA \\
CellSens Dimension & Olympus GmbH, Hamburg \\
Endnote X5 & Thomson ISI ResearchSoft, California, USA \\
FlowJo & Tree Star Inc., Oregon, USA \\
GraphPad Prism 6 & GraphPad Software, Inc., La Jolla, CA, USA \\
Intas GDS & Intas Science Imaging Instruments GmbH, Göttingen \\
Gen5 1.11 & BioTek Instruments, Inc., Bad Friedrichshall \\
Microsoft Office & Microsoft Co., Redmont, USA \\
Fiji & (Schindelin et al., 2012) \\
Quantum FX $\mu$ CT (SimpleViewer) & PerkinElmer Health Sciences, Hopkinton USA \\
SDS 2.2 & Applied Biosystems, Darmstadt \\
Statistica 10 & StatSoft GmbH, Hamburg \\
\hline
\end{tabular}

\subsection{Databases}

Table 13: Databases to retrieve information

\begin{tabular}{ll}
\hline Database & Homepage \\
\hline BasicLocalAlignmentSearchTool (BLAST) & http://blast.ncbi.nlm.nih.gov/Blast.cg \\
Ensembl & http://www.ensembl.org/index.html \\
MGI 3.43-mouse genome informatics & http://www.informatics.jax.org/ \\
National Center for Biotechnology Information (NCBI) & http://www.ncbi.nlm.nih.gov/ \\
\hline
\end{tabular}




\section{METHODS}

\subsection{Molecular biology methods}

\subsubsection{Nucleic acid isolation}

\subsubsection{Isolation of genomic DNA}

For isolation of genomic DNA (gDNA) from mouse tissue, tail biopsies were incubated overnight at $55^{\circ} \mathrm{C}$ in $400 \mu \mathrm{l}$ of STE buffer containing $0.5 \mathrm{mg} / \mathrm{ml}$ Proteinase $\mathrm{K}$. Afterwards undigested tissue debris was removed by centrifugation for $10 \mathrm{~min}$ at $13000 \mathrm{rpm}$. The supernatant was transferred into a fresh reaction tube containing $1 \mathrm{ml}$ cold $99 \% \mathrm{EtOH}$ and shook thoroughly in order to precipitate the nucleic acid. The gDNA was pelleted by centrifugation (25 min, $13000 \mathrm{rpm}$ ), washed with $500 \mu \mathrm{l} 70 \%$ ethanol and centrifuged again (10 min, $13000 \mathrm{rpm})$. After drying of the gDNA for $10 \mathrm{~min}$ at $55^{\circ} \mathrm{C}$, gDNA was solved in $125 \mu \mathrm{ddH}_{2} \mathrm{O}$ for $10 \mathrm{~min}$ at $42{ }^{\circ} \mathrm{C}$ and $1400 \mathrm{rpm}$ and stored at $-20{ }^{\circ} \mathrm{C}$ for further analyses.

\subsubsection{Isolation of total RNA from cell culture}

Extraction of total RNA from cell culture was performed by using TRIzol reagent corresponding to the manufacturer's instructions. If not stated otherwise all steps were performed on ice to avoid degradation of the RNA. Briefly, the cells were washed with cold PBS and detached by adding $1 \mathrm{ml}$ TRIzol. Samples were transferred into $2 \mathrm{ml}$ reaction tubes, vortexed for $2 \mathrm{~min}$ and incubated for $5 \mathrm{~min}$ at room temperature (RT). After addition of $200 \mu \mathrm{l}$ chloroform and vortexing for $15 \mathrm{sec}$, samples were incubated for another $3 \mathrm{~min}$ at RT. After phase separation by centrifugation $\left(10 \mathrm{~min}, 13000 \mathrm{rpm}, 4{ }^{\circ} \mathrm{C}\right.$ ) the upper aqueous phase (containing the RNA) was transferred into $1 \mathrm{ml}$ isopropyl alcohol and precipitated overnight at $-20{ }^{\circ} \mathrm{C}$. Afterwards the samples were centrifuged ( $30 \mathrm{~min}, 13000 \mathrm{rpm}, 4{ }^{\circ} \mathrm{C}$ ), the supernatant was removed and the pellet was washed two times with $500 \mu 170 \% \mathrm{DNase} / \mathrm{RNase}$-free $\mathrm{EtOH}\left(-20{ }^{\circ} \mathrm{C}\right)$ by centrifugation (10 min, $13000 \mathrm{rpm}, 4{ }^{\circ} \mathrm{C}$ ). The pellet was dried for $10 \mathrm{~min}$ at $\mathrm{RT}$ and dissolved in DNase/RNase-free $\mathrm{H}_{2} \mathrm{O}$ for $5-10$ min at $56{ }^{\circ} \mathrm{C}$. The RNA was stored at $-80{ }^{\circ} \mathrm{C}$ before use. 


\subsubsection{Photometric quantification of nucleic acids}

DNA and RNA concentration was quantified using a Spectrophotometer (NanoDrop 8000). Nucleic acid quantity was assessed by determination of the optical density at $260 \mathrm{~nm}\left(\mathrm{OD}_{260}\right)$, because an $\mathrm{OD}_{260}$ of 1.0 equates $50 \mu \mathrm{g} / \mathrm{ml}$ pure DNA or $40 \mu \mathrm{g} / \mathrm{ml}$ pure RNA. The DNA or RNA concentrations were calculated according to the following formula:

$$
\text { Concentration }(\mathrm{ng} / \mu \mathrm{l})=\mathrm{OD}_{260} \times 50(\mathrm{DNA}) \text { or } 40(\mathrm{RNA})
$$

Because the $\mathrm{OD}$ at $280 \mathrm{~nm}\left(\mathrm{OD}_{280}\right)$ provides the protein concentration of the sample the ratio $\mathrm{OD}_{260} / \mathrm{OD}_{280}$ was used to evaluate the purity of the samples. Pure DNA and RNA preparations are generally recognized by $\mathrm{OD}_{260} / \mathrm{OD}_{280}$ values of $\sim 1.8$ and $\sim 2.0$, respectively.

\subsubsection{Reverse transcription of RNA (cDNA synthesis)}

For synthesis of complementary DNA (cDNA), $2 \mu \mathrm{g}$ of RNA were reversely transcribed using the SuperScriptII Reverse Transcriptase System in a final reaction volume of $20 \mu$. First the RNA was incubated with $250 \mathrm{ng}$ hexamers for $10 \mathrm{~min}$ at $70{ }^{\circ} \mathrm{C}$. Next $10 \mathrm{mM}$ DTT with $0.5 \mathrm{mM}$ dNTPs dissolved in 1st strand buffer, were added and incubated at RT for $10 \mathrm{~min}$. Following pre-warming to $42{ }^{\circ} \mathrm{C}$ for $2 \mathrm{~min}, 1 \mu \mathrm{l}$ of SuperScriptII $(100 \mathrm{U} / \mu \mathrm{l})$ was added and the mixture was incubated for $1 \mathrm{~h}$ at $42{ }^{\circ} \mathrm{C}$. Afterwards the synthesis reaction was stopped at $70{ }^{\circ} \mathrm{C}$ for $10 \mathrm{~min}$. Under the assumption that the reverse transcription reaction is $50 \%$ efficient, the final concentration of cDNA was $50 \mathrm{ng} / \mu \mathrm{l}$.

\subsubsection{Polymerase chain reaction (PCR)}

\subsubsection{PCR-based genotyping of mouse tail gDNA}

PCR-based amplification of gDNA was carried out in reaction volumes of 10 or $20 \mu$ per assay and performed with the reagents and final concentrations shown in Table 14. 
Table 14: Reaction mixture for genotyping of mouse tail gDNA

\begin{tabular}{ll}
\hline Concentration & Component \\
\hline $10-100 \mathrm{ng}$ & gDNA template \\
$0.5 \mu \mathrm{M}$ & sequence-specific forward DNA oligonucleotide (forward primer) \\
$0.5 \mu \mathrm{M}$ & sequence-specific reverse DNA oligonucleotide (reverse primer) \\
$0.2 \mathrm{mM}$ & dNTP-Mix \\
$10 \%(\mathrm{v} / \mathrm{v})$ & Cresol \\
$1 \mathrm{x}$ & Polymerase buffer \\
$0.1 \mathrm{U}$ & Taq-Polymerase \\
\hline
\end{tabular}

The primer sequences used for genotyping are given in Table 9.

PCR steps were the following:

1) Initiation step for 4 min at $95{ }^{\circ} \mathrm{C}$

2) Denaturation step for $30 \mathrm{sec}$ at $95^{\circ} \mathrm{C}$

3) Annealing step for $75 \mathrm{sec}$ at $60{ }^{\circ} \mathrm{C}$

4) Elongation step for $90 \mathrm{sec}$ at $72{ }^{\circ} \mathrm{C}$

The steps 2) to 4) were repeated for 35 cycles. The reaction was terminated by a final elongation step for 5 min at $72{ }^{\circ} \mathrm{C}$. The samples were subsequently analyzed by agarose gel electrophoresis.

\subsubsection{Quantitative Real-Time PCR (qRT-PCR)}

Gene expression was analyzed using SYBR Green based assays. Primers for amplification of the target transcripts are listed in Table 10. The primer pairs used in this thesis were intron spanning, except of the primers for $18 S$ and $h M Y O D$ that were located within a single exon. The assays were performed in a total reaction mixture of $10 \mu \mathrm{l}$ using the reagents listed in Table 15.

Table 15: Reaction mixture for qRT-PCR

\begin{tabular}{ll}
\hline Amount & Component \\
\hline $2 \mu \mathrm{l}$ & cDNA template \\
$0.4 \mu \mathrm{M}$ & sequence-specific forward DNA oligonucleotide (forward primer) \\
$0.4 \mu \mathrm{M}$ & sequence-specific reverse DNA oligonucleotide (reverse primer) \\
$0.41 \mu \mathrm{l}$ & $\mathrm{ddH}_{2} \mathrm{O}$ \\
$0.4 \mu \mathrm{l}$ & $\mathrm{SYBR}$ Green \\
\hline
\end{tabular}

Gene expression levels were calculated using the standard curve method. For quantification of the RNA levels a standard curve was generated by 5 fold serial dilutions of $20 \mathrm{ng}$ cDNA (80 pg 


\section{METHODS}

cDNA for $18 S$ rRNA) of a standard sample. Standard samples were derived from tissue or cells known to express the respective gene. The logarithm of the amount of cDNA for each dilution was plotted against the measured cycle threshold value of the standard curve. The derived linear trend line with the corresponding equation $(y=m x+b)$ served to interpolate the amount of cDNA in each sample. Finally, the transcript levels of each sample were normalized to the corresponding expression of the housekeeper gene $18 S$ rRNA. The samples were measured in triplicates. Analysis was done using the SDS 2.2, Microsoft Excel and GraphPad Prism 6 software.

\subsubsection{Agarose gel electrophoresis}

Gel electrophoresis was performed to separate DNA fragments according to their specific size. Agarose gels composed of $1 \%(\mathrm{w} / \mathrm{v})$ agarose in $1 \mathrm{x}$ TBE buffer were prepared by boiling for 2-3 min at $1000 \mathrm{~W}$ in a microwave. After cooling, 5-7 drops of 0.07\% ethidium bromide were added to the liquid gels, which were subsequently hardened in the electrophoresis chamber. Gels were put in TBE buffer in the electrophoresis chamber, DNA samples and an appropriate DNA ladder were loaded onto the gels. The gels were run constantly at $100 \mathrm{~V}$. For documentation an UV transilluminator was used.

\subsection{Cell biology methods}

\subsubsection{Cell culture}

An overview about the used cell lines and respective culture conditions is given in Table 8. All RMS cell lines were cultured in an incubator at constant $37{ }^{\circ} \mathrm{C}, 5 \% \mathrm{CO}_{2}$ and $95 \%$ humidity. Every third to forth day the media were refreshed and cells were splitted when reaching 80 to $90 \%$ confluence. Splitting of the cells was performed by detaching the cells with 1-3 ml of TrypLE Express. When the cells started to detach the reaction was stopped by adding of FCS containing medium. The cells were transferred to a new culture plate containing fresh medium. For in vitro assays, certain numbers of RMS cells were seeded in different plates (see Table 16) and incubated with drugs (see Table 4) as indicated in the respective experiments. 
Table 16: Cell culture preparations for in vitro assays

\begin{tabular}{lll}
\hline Assay & Format & Seeded cells/well \\
\hline qRT-PCR, Apoptosis & 6-well plates & $10^{5}$ (ERMS) or $15 \times 10^{4}$ cells (ARMS) \\
Proliferation, Viability & 96-well plates & 4000 \\
Western blot & $10 \mathrm{~cm}$-culture dishes & $10^{6}$ (ERMS) or $15 \times 10^{5}$ cells (ARMS) \\
\hline
\end{tabular}

\subsubsection{Cryopreservation of eukaryotic cells}

For long term storage in liquid nitrogen, RMS cell lines that were $90 \%$ confluent were rinsed with PBS and detached as described. The cells were transferred into a $15 \mathrm{ml}$ reaction tube and pelleted by centrifugation $\left(5 \mathrm{~min}, 300 \mathrm{x} \mathrm{g}, 4{ }^{\circ} \mathrm{C}\right.$ ). Afterwards the cells were resuspended in $10 \mathrm{ml}$ culture medium supplemented with $5 \%$ DMSO. Subsequently, $1 \mathrm{ml}$ aliquots were prepared and frozen in cryo tubes at $-80{ }^{\circ} \mathrm{C}$ in the freezing device Mr. Frosty ${ }^{\mathrm{TM}}$. After $16 \mathrm{~h}$ the cells were transferred in liquid nitrogen.

For thawing, cells were rapidly warmed and transferred to $10 \mathrm{ml}$ medium, pelleted (5 min, $300 \mathrm{x} \mathrm{g}, 4^{\circ} \mathrm{C}$ ) and resuspended in fresh culture medium. Cells were subsequently transferred to a cell culture dish and stored in an incubator. To ensure a complete elimination of DMSO, the medium was immediately replaced the next day.

\subsubsection{Isolation and culture of primary cells}

Primary RMS cells were isolated from RMS bearing $\mathrm{Ptch}^{+/-}$mice. Animals were euthanized and tumors were excised using scalpels and scissors followed by washing in PBS. One part of the tumor was embedded in paraffin in order to confirm the identity as RMS by haematoxylin eosin (HE) staining. The remaining tumor tissue was chopped using a razor blade and incubated with $3 \mathrm{mg} / \mathrm{ml}$ collagenase $\mathrm{H}$ in DMEM (50 min, $1000 \mathrm{rpm}, 37^{\circ} \mathrm{C}$ ). The resulting cell suspension was triturated through a $1 \mathrm{ml}$ pipette. After sedimentation of undigested tissue, the tumor cells within the supernatant were separated using a cell strainer and centrifuged for $5 \mathrm{~min}$ at $300 \mathrm{xg}$ and $4{ }^{\circ} \mathrm{C}$. The pellet was solved in DMEM supplemented with $10 \%$ FCS and $1 \%$ P/S and plated on collagen coated 6-well plates for further analyses. The next day the cells were washed to remove remaining blood cells and cell debris. Experiments were started immediately or within the next three days. 


\subsubsection{Proliferation assay}

Cellular proliferation was analyzed using the 5-Bromo-2-Deoxyuridine (BrdU) cell proliferation kit according to the manufacturer's instructions. Briefly, 4000 cells were seeded in 96-well plates and were incubated for $24 \mathrm{~h}$ with the respective inhibitors in the presence of BrdU. After fixation of the cells and denaturation of the DNA for $30 \mathrm{~min}$, peroxidase coupled BrdU antibody (anti-BrdU-POD) was added for $1 \mathrm{~h}$. Subsequently, cells were washed thoroughly followed by adding peroxidase substrate. Measurement of BrdU incorporation via luminescence was performed in a microplate reader. The analyzed luminescence is proportional to newly synthesized DNA and hence to the proliferation of the cells. All samples were measured in triplicates, analyzed and plotted using GraphPad Prism 6.

\subsubsection{Analysis of cellular proliferation by counting}

In order to confirm the results from the BrdU incorporation assay, proliferation was estimated by counting of the cells. For that purpose $10^{5}$ cells/well (ERMS) or $15 \times 10^{4}$ cells/well (ARMS) were seeded in 6-well plates followed by incubation with the corresponding inhibitors for $24 \mathrm{~h}$. Cells were washed with PBS, detached with $1 \mathrm{ml}$ TrypLE Express/well, transferred into a $50 \mathrm{ml}$ reaction tube and pelleted by centrifugation $\left(5 \mathrm{~min}, 300 \mathrm{xg}, 4{ }^{\circ} \mathrm{C}\right)$. After removal of the supernatant, the pellet was resuspended in $1 \mathrm{ml}$ culture medium. Subsequently, the cell suspension was diluted in trypan blue solution to distinguish between live and dead cells. Counting was performed using a Neubauer counting chamber.

\subsubsection{Cell viability assay}

Cellular viability was measured using the tetrazolium salt WST-1. RMS cell lines were seeded in 96-well plates (4000/well) and incubated for $24 \mathrm{~h}$ with the respective drugs. $4 \mathrm{~h}$ before analysis, $10 \mu \mathrm{l}$ WST-1 reagent/well (equates to a final 1:10 dilution of WST-1) was added as recommended by the manufacturer. WST-1 is cleaved to a soluble formazan dye in the presence of $\mathrm{NAD}(\mathrm{P}) \mathrm{H}$ in viable cells and the amount of produced formazan dye (quantified in a microplate reader at a wavelength of $450 \mathrm{~nm}$ ) correlates with the metabolic activity of the cells. All samples were measured in triplicates, analyzed and graphed using GraphPad Prism 6. 


\subsubsection{Apoptosis assay}

Cellular apoptosis was analyzed via flow cytometry after staining of the cells with AnnexinV coupled with fluorescein isothiocyanate (FITC) and Propidium Iodide (PI) according to manufacturer's instructions. During early apoptosis, the membrane phospholipid phosphatidylserine (PS) is translocated from the inner to the outer leaflet of the plasma membrane. AnnexinV which specifically binds PS in a $\mathrm{Ca}^{2+}$ dependent manner can therefore detect apoptotic cells at an early stage. Because ongoing loss of membrane integrity also leads to binding of AnnexinV to PS in the cytosolic membrane leaflet, AnnexinV was combined with PI that can intercalate into the DNA of dead and apoptotic cells with damaged cell membranes. This allows for differentiation of early and late cell death. Thus, viable cells are AnnexinV negative and PI negative (AnnexinV-/PI-), early apoptotic cells are AnnexinV positive and PI negative (AnnexinV+/PI-), late apoptotic cells are AnnexinV positive and PI positive (AnnexinV+/PI+) and AnnexinV negative and PI positive (Annexin V-/PI+) cells are necrotic.

For the analysis, $10^{5}$ cells/well (ERMS) or $15 \times 10^{4}$ cells/well (ARMS) were seeded in 6-well plates and incubated with the respective drugs for $48 \mathrm{~h}$. Cells were rinsed in PBS, detached with $1 \mathrm{ml}$ accutase/well, transferred into a $15 \mathrm{ml}$ reaction tube and pelleted by centrifugation $(5 \mathrm{~min}$, $300 \mathrm{x}$ g, $4{ }^{\circ} \mathrm{C}$ ). After washing with PBS the cells were incubated with $100 \mu \mathrm{l}$ AnnexinV binding buffer supplemented with $2 \mu \mathrm{l}$ AnnexinV-FITC for $10 \mathrm{~min}$, followed by incubation with PI for 5 min. Before measurement on a FACS Calibur $300 \mu 1$ of AnnexinV binding buffer was added to the cell suspension. Incubations were conducted at RT and under exclusion of light. Measurement of fluorescence was performed in channel FL-1 for AnnexinV-FITC (emission maximum of FITC is about $519 \mathrm{~nm}$ ) and in channel FL-3 for PI (emission maximum of PI is about $617 \mathrm{~nm}$ ). The samples were measured in duplicates, analyzed and displayed using the software FlowJo, Microsoft Excel and GraphPad Prism 6.

\subsection{Protein chemistry and immunohistochemistry (IHC)}

\subsubsection{Protein isolation from cell culture}

For protein isolation from cell culture, cells were washed and scraped in $2 \mathrm{ml}$ cold PBS using a cell scraper and centrifuged $\left(5 \mathrm{~min}, 1800 \mathrm{rpm}, 4{ }^{\circ} \mathrm{C}\right)$. After removing of the supernatant, the pellet was resuspended in $800 \mu \mathrm{l}$ PBS and transferred into a $1.5 \mathrm{ml}$ reaction tube in order to perform a second washing step. Cell membranes were disrupted by shock freezing in liquid 


\section{METHODS}

nitrogen and thawing on ice for 20 min followed by incubation with $80-100 \mu 1$ lysis buffer (supplemented with $500 \mu \mathrm{M}$ PMSF und $2 \mathrm{mM}$ DTT) for $30 \mathrm{~min}$ on ice. Subsequently the lysates were centrifuged $\left(30 \mathrm{~min}, 13000 \mathrm{rpm}, 4{ }^{\circ} \mathrm{C}\right.$ ) and the soluble proteins in the supernatant were transferred to a new $1.5 \mathrm{ml}$ reaction tube and stored at $-80{ }^{\circ} \mathrm{C}$ until use.

\subsubsection{Determination of protein concentration}

The protein concentration was measured using the Pierce BCA Protein Assay Kit according to manufacturer's instructions. This method is based on bicinchoninic acid (BCA) for the colorimetric detection and quantitation of total protein. Through reduction of $\mathrm{Cu}^{2+}$ to $\mathrm{Cu}^{1+}$ by the proteins in an alkaline environment, BCA reacts with $\mathrm{Cu}^{1+}$ to form a purple colored reaction product. The amount of the produced complex is proportional to increasing protein concentrations in the samples and can be measured at $540 \mathrm{~nm}$ with a microplate reader.

Triplicates of $1 \mu \mathrm{l}$ of each protein sample were pipetted in a 96-well plate. Known concentrations $(0-2000 \mu \mathrm{g} / \mathrm{ml})$ of BSA were used to create a standard curve for calculation of the protein concentrations. After addition of $200 \mu \mathrm{l} /$ well BCA reagent and an incubation for $30 \mathrm{~min}$ at $37^{\circ} \mathrm{C}$, the plate was analyzed in the microplate reader.

\subsubsection{Western blot}

For western blot analysis, protein lysates were denatured in SDS loading buffer for 5 min at $96{ }^{\circ} \mathrm{C}$ and $450 \mathrm{rpm}$ in a shaker. Afterwards $30-40 \mu \mathrm{g}$ proteins were loaded on $4-12 \%$ Bis-Tris Gels (NuPAGE Novex) and electrophoresed in running buffer (NuPAGE MES SDS) at $160 \mathrm{~mA}$, $160 \mathrm{~V}$ and $100 \mathrm{~W}$ for $1.5-2 \mathrm{~h}$. A protein marker (SeeBlue ${ }^{\circledR}$ Plus2 Pre-Stained) with proteins of defined molecular weight, was loaded to estimate the size of the protein bands later. After separation of the proteins during gel electrophoresis, they were blotted onto a nitrocellulose membrane. The transfer of the proteins from the gel to the membrane was performed by semi-dry blotting in blotting buffer at $120 \mathrm{~mA}, 20 \mathrm{~V}$ and $100 \mathrm{~W}$ for $80 \mathrm{~min}$. After blocking with $5 \%(\mathrm{w} / \mathrm{v})$ milk powder/PBST for $1 \mathrm{~h}$ at RT followed by washing three times in PBST for $10 \mathrm{~min}$, the membrane was incubated with primary antibodies (see Table 11) at $4{ }^{\circ} \mathrm{C}$ overnight. The next day, the membrane was washed again three times for $10 \mathrm{~min}$ in PBST and subsequently incubated with HRP conjugated secondary antibodies for $1 \mathrm{~h}$ at RT (see Table 11). After additional washing steps in PBST the detection reagent ECL was pipetted onto the membrane, incubated for $1 \mathrm{~min}$ at RT and carefully removed before the detection was accomplished with a 
Fluorchem Q Imaging system. All primary antibodies were dissolved in BSA-azide/PBST (except of IGF2 which was dissolved in $5 \%$ milk powder/PBST) and all secondary antibodies were dissolved in $5 \%$ milk powder/PBST.

\subsubsection{Haematoxylin eosin (HE) staining}

For HE staining, tissue fixed in $4 \%$ PFA was embedded in paraffin and cut into 4-5 $\mu \mathrm{m}$ sections and mounted onto glass slides. The sections were then deparaffinized two times in xylene for $10 \mathrm{~min}$ and subsequently rehydrated by descending ethanol solutions (99\% to $70 \%$ ). After thorough washing with $\mathrm{ddH}_{2} \mathrm{O}$, slides were transferred in haematoxylin solution for $15 \mathrm{~min}$. Coloring occurred after washing with lukewarm tap water for at least $5 \mathrm{~min}$. Slides were then quickly placed in $1 \%$ eosin solution for a maximum of $20 \mathrm{sec}$ and washed again with $\mathrm{ddH}_{2} \mathrm{O}$. The sections were dehydrated using ascending ethanol solutions (70\% to $99 \%$ ). Afterwards the slides were placed again in xylene prior mounting in pertex. Finally in order to harden the mounting medium, the slides were incubated at $55^{\circ} \mathrm{C}$ for at least $20 \mathrm{~min}$.

\subsubsection{Ki67-staining}

For immunohistochemical staining of Ki67 positive cells, sections of paraffin embedded tumors were mounted onto superfrost slides. Tissue was deparaffinized and rehydrated as described above (see 5.3.4). After rinsing in $\mathrm{ddH}_{2} \mathrm{O}$, antigen retrieval was performed by boiling the slides in citric acid buffer ( $\mathrm{pH}$ 6.0) once for $4 \mathrm{~min}$ and four times for $3 \mathrm{~min}$ at $600 \mathrm{~W}$ in a microwave. After cooling down to RT and washing two times for 2 min with TBS, the slides were blocked for 20 min with $3 \% \mathrm{H}_{2} \mathrm{O}_{2}$ to inhibit endogenous peroxidases. Then the sections were washed for 5 min in $\mathrm{ddH}_{2} \mathrm{O}$ and rinsed with TBS. The following steps were performed in moist chambers. In order to block nonspecific antibody binding, slides were incubated with $0.2 \%$ casein in TBS for 20 min at RT. Incubation with the primary antibody in TBS solution was performed overnight at $4{ }^{\circ} \mathrm{C}$. After washing three times with TBST, the slides were incubated with the secondary antibody for $30 \mathrm{~min}$ at RT. Prior adding of diaminobenzidine chromogen for $10 \mathrm{~min}$ at RT, the slides were again washed in TBST. To stop the reaction, slides were washed with $\mathrm{ddH}_{2} \mathrm{O}$. Counterstaining with haematoxylin was performed for $5 \mathrm{sec}$ followed by washing with lukewarm tap water for 5 min and sections were mounted with glycergel.

Quantification of Ki67 positive cells was done on a microscope (Olympus BX 60) using CellSens dimension software. For analyzing and counting of the cells, 11 images per section 


\section{METHODS}

were taken at a $200 \mathrm{x}$ magnification. The pictures were analyzed by the freely available software Fiji (Schindelin et al., 2012). All nuclei per image as well as Ki67 positive cells were automatically counted (if tumors were very small, at least 6000 cells per section were counted). In addition manual counting of Ki67 positive cells was performed to control and confirm the automatic counting. The ratio of Ki67 positive cells/all nuclei per picture was determined and used for further calculations.

\subsection{Animal experiments}

\subsubsection{Mouse lines}

The wild type inbred mouse strains C57BL/6N (B6) and Balb/c (Balb) were bred in-house or purchased from Jackson Laboratories (Bar Harbor, USA) or Charles River Laboratories (Sulzfeld, Germany). B6-Ptch ${ }^{\text {neo67/+ }}$ mice harbour a heterozygous deletion of exon 6 and 7 within the Ptch gene. For in vivo studies heterozygous B6-Ptch ${ }^{\text {neo67/+ }}$ mice were bred to Balb mice. The resulting B6xBalbc-Ptch ${ }^{\text {neo67 }}$ mice were used for further studies and were henceforth named $\mathrm{Ptch}^{+/-}$mice. All experiments using animals were performed in agreement with all relevant legal and ethical requirements.

\subsubsection{Breeding of mice}

The mouse strains used in this thesis were bred and housed in the animal facility of the Institute of Human Genetics, University of Göttingen, Germany. Animals were kept in Makrolon cages type II and III. Mice were exposed to a $12 \mathrm{~h}$ light-dark cycle (light period: 6 a.m.- 6 p.m.), a temperature of $20 \pm 2{ }^{\circ} \mathrm{C}$ and a relative humidity of $50 \pm 10 \%$. Rodent pellets and tap water were given ad libitum.

\subsubsection{Tail biopsy and genotyping of mice}

To identify the genotype and to mark the mice, tail clipping $(0.2 \mathrm{~cm}$ cut from the tip of the tail $)$ and ear marking were done at 4 weeks of age by the staff of the animal facility. Genotyping was performed by isolation of gDNA from tail tissue followed by PCR using the primers and conditions shown in Table 9. 


\subsubsection{Monitoring of RMS bearing mice}

In order to record the occurrence and estimate the size of RMS, $\mathrm{Ptch}^{+/}$mice were monitored once a week manually by palpation and carefully viewing.

\subsubsection{Formulation of the drugs for in vivo treatment}

HhA and GDC-0941 were obtained from Genentech. For in vivo treatment both drugs were dissolved in methyl cellulose tween (MCT) as recommended by the manufacturer. In short, MCT powder was first solved in $40 \mathrm{ml}$ hot $\mathrm{ddH}_{2} \mathrm{O}\left(80{ }^{\circ} \mathrm{C}-90{ }^{\circ} \mathrm{C}\right)$ while stirring. Then $40 \mathrm{ml}$ cold $\mathrm{ddH}_{2} \mathrm{O}$ was slowly added. The resulting MCT suspension was refrigerated overnight. The next day, the clear solution was equilibrated to RT prior and then tween-80 was added. After filling up to $100 \mathrm{ml}$ with $\mathrm{ddH}_{2} \mathrm{O}$, the MCT solution was sterile filtered and stored at $2-8{ }^{\circ} \mathrm{C}$ no longer than a month before use. The solution was used as the vehicle in the control group and to dissolve HhA and GDC-0941. The stock solution for GDC-0941 was stored for a maximum of three days at RT. HhA was prepared directly before oral application.

\subsubsection{Treatment of $\mathrm{Ptch}^{+/-}$mice with $\mathrm{HhA}$ and GDC-0941}

Tumor bearing mice were randomized into four groups. Mice were treated daily for five weeks with the same dosing volume of vehicle MCT, $100 \mathrm{mg} / \mathrm{kg} \mathrm{HhA,} 75 \mathrm{mg} / \mathrm{kg}$ GDC-0941 or with the combination of HhA plus GDC-0941. Oral treatment was performed using feeding tubes. For the combination treatment the drugs were administered separately with an interjacent time span of at least $5 \mathrm{~h}$. This was necessary to avoid potential drug-drug interactions or complexations, which could have lowered the intestinal resorption and hence the therapeutic efficacy of the drugs. During the treatment mice were weighed twice a week and their physical condition was monitored. The animals were kept until completion of the study, than all mice were sacrificed and tumors were excised. However, animals were sacrificed earlier if they lost $20 \%$ of their body weight, were at poor general condition or if the tumor exceeded a size of $1.5 \mathrm{~cm}$. The tumors tissue was formalin fixed and embedded in paraffin for HE staining and IHC. Due to the small size of the treated tumors (especially after combination therapy) it was not possible to freeze tumor tissue for RNA or protein isolation. 


\subsubsection{Measurement of tumor growth using volumetric computer tomography (VCT)}

The tumor growth during in vivo treatment with HhA and GDC-0941 was analyzed using VCT. The tumor volume was measured at three time points; at therapy start, two weeks after therapy begin and at end of the treatment. Mice were anaesthetized with isoflurane vapor before and during the scan procedure. In order to visualize the tumors $200 \mu 1$ of contrast agent Imeron 300 (Struffert et al., 2010) were injected into the tail vein approximately $30 \mathrm{sec}$ prior imaging. A lowdose laboratory animal CT system (QuantumFX, Perkin Elmer) (Osborne et al., 2012) was used for in vivo imaging of tumor bearing mice. The data sets were acquired using a field of few of 4 $\mathrm{x} 4 \mathrm{~cm}^{2}$ centered on the tumor site. The system was operated at $70 \mathrm{kVp}, 200 \mu \mathrm{A}$ using $17 \mathrm{sec}$ total scanning time in a $360^{\circ}$ rotation mode. Data sets were reconstructed with a voxel size of $\mathrm{XYZ} \mu \mathrm{m}$.

\subsection{Statistics}

Unless indicated otherwise, statistical and graphical analyses were conducted using the programs GraphPad Prism 6, MS office Excel or Statistica 10. Data were considered significant if $\mathrm{p}$ values were $<0.05$. When comparing two samples, statistical differences were analyzed using Student's t-test. In order to analyze statistical differences for experiments using more than two treatment schemes Tukey's test in conjunction with ANOVA was applied.

Statistical analysis of the in vivo data was done with the aid of Albert Rosenberger (department of genetic epidemiology, university medical center Göttingen). Differences in tumor growth between the cohorts were analyzed using a mixed, linear model. The logarithm of the tumor size was generated to achieve the assumption of a Gaussian distribution. In few cases more than one tumor per mouse was observed, hence the factor mouse was considered as random effect. The expected tumor size per treatment group was adjusted to the tumor size at time points of the therapy begin, calculated and tested. Multiple testing was corrected by the method of Tukey. 


\section{RESULTS}

\subsection{Effects of four SMO antagonists on RMS cell lines}

In order to investigate the impact of SMO inhibitors on RMS cells, the potential antitumoral effects of the approved SMO inhibitor GDC-0449 was compared with that of LDE225, HhA and cyclopamine. The effects were analyzed in the ERMS cell lines RD and RUCH-2 and in the ARMS cell lines RMS-13 and Rh41.

\subsubsection{Modulation of gene expression by GDC-0449, LDE225, HhA and cyclopamine}

Initially, the effects of GDC-0449, LDE225, HhA, and cyclopamine on HH pathway inhibition (indicated by GLII expression), on IGF2 expression and on modulation of the differentiation status of the four RMS cell lines were examined.

\subsubsection{Effects on GLII expression}

As known from previous studies, all four RMS cell lines express major components of the HH signaling pathway including GLII and $P T C H$, which are the main target genes of $\mathrm{HH}$ signaling (Graab et al., 2015; Ridzewski et al., 2015). This indicates that the RMS cell lines used in this thesis show HH pathway activity. Since the most reliable indicator for active $\mathrm{HH}$ signaling is GLII mRNA expression (reviewed in (Scales and de Sauvage, 2009)), the effects of the SMO antagonists on the pathway's activity was analyzed by GLII qRT-PCR. First, the optimal concentrations under which each inhibitor may block HH signaling were determined.

In the ERMS cell line RD a significant inhibition of GLI1 expression was observed for 1, 10 and $50 \mu \mathrm{M}$ GDC-0449 and LDE225 (Figure 5). A significant decrease of GLI1 expression was also revealed for $1-50 \mu \mathrm{M}$ HhA. Interestingly, $30 \mu \mathrm{M}$ of GDC-0449 or LDE225 did not block HH signaling activity, but rather restored $\mathrm{HH}$ signaling activity to basal level or above. Moreover, a triphasic change in GLI1 expression was observed after treatment with GDC-0449, LDE225 or HhA i.e. a decrease at $10 \mu \mathrm{M}$, a re-increase at $30 \mu \mathrm{M}$ compared to $10 \mu \mathrm{M}$ and again a decrease at $50 \mu \mathrm{M}$. Cyclopamine was not able to decrease GLII expression indeed it rather enhanced GLII expression, which was significant at a concentration of $1 \mu \mathrm{M}$ (Figure 5). 


\section{RESULTS}

In RUCH-2 cells GDC-0449 did not significantly modulate GLI1 expression at any concentration (Figure 5). However, LDE225 and HhA decreased GLII transcription at 30 and $50 \mu \mathrm{M}$ and at 10 and $50 \mu \mathrm{M}$, respectively. Cyclopamine increased GLII expression at concentrations of $0.1,1$ and $5 \mu \mathrm{M}$, which was similar to its action in RD cells (Figure 5).

In RMS-13 cells, which show amplification of GLI1 (Roberts et al., 1989), the SMO inhibitors did not decrease GLII mRNA levels at any applied concentration. In contrast, GLII expression was rather stimulated. This was especially obvious for LDE225, HhA and cyclopamine (Figure $5)$.

In Rh41 cells the dose response analyses revealed that GDC-0449 moderately inhibited GLII expression at any used concentration (Figure 5). LDE225 decreased GLII expression at concentrations of 10 and $30 \mu \mathrm{M}$, but increased it at a concentration of $50 \mu \mathrm{M}$. HhA blocked GLII transcription at 0.1 and $1 \mu \mathrm{M}$, while higher concentrations also restored or increased GLII expression levels. Cyclopamine never inhibited GLII expression, but activated GLII expression significantly at 1-10 $\mu \mathrm{M}$ (Figure 5).

Together, these findings indicate that tumor intrinsic HH pathway activity (evaluated by GLII expression) in ERMS and ARMS cell lines can be modulated by treatment with SMO antagonists. However, the data show that the drugs exert heterogeneous effects on GLII expression despite blocking the same molecular target (i.e. SMO). In addition, the responsiveness of the cell lines to the SMO inhibitors is very variable. Except for cyclopamine, which never repressed HH signaling activity in any of the used RMS cell lines, all other SMO antagonists induced effects that differed from cell line to cell line. Moreover, the data demonstrate that SMO inhibitors (depending on their concentration) might paradoxically induce GLII transcription in some RMS cell lines. 
RD

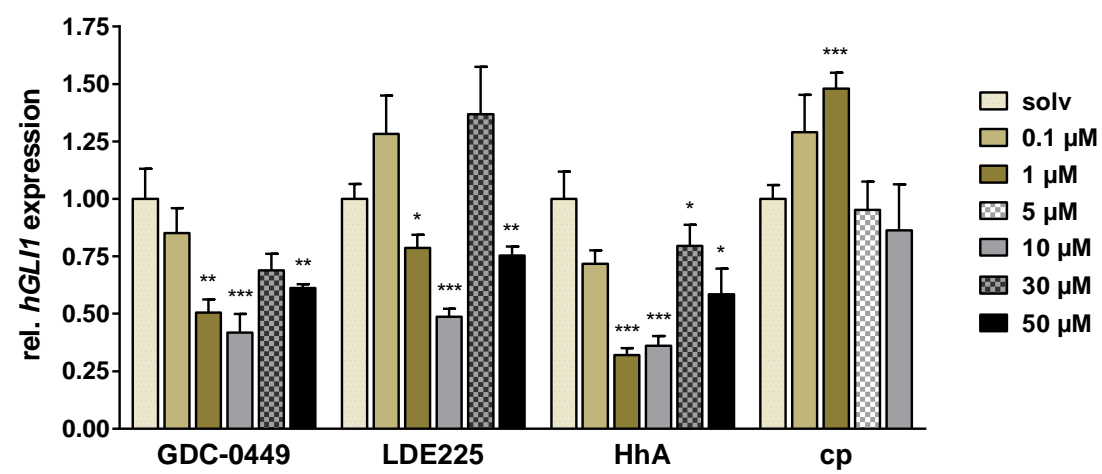

RUCH-2

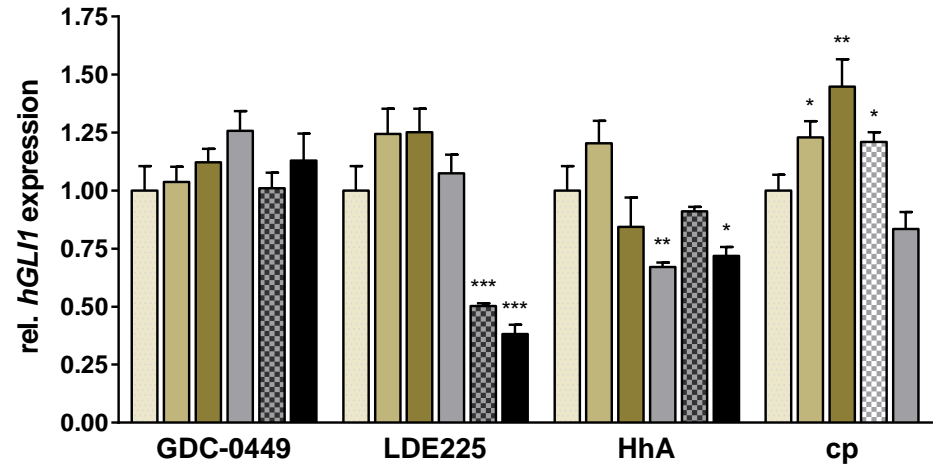

RMS-13

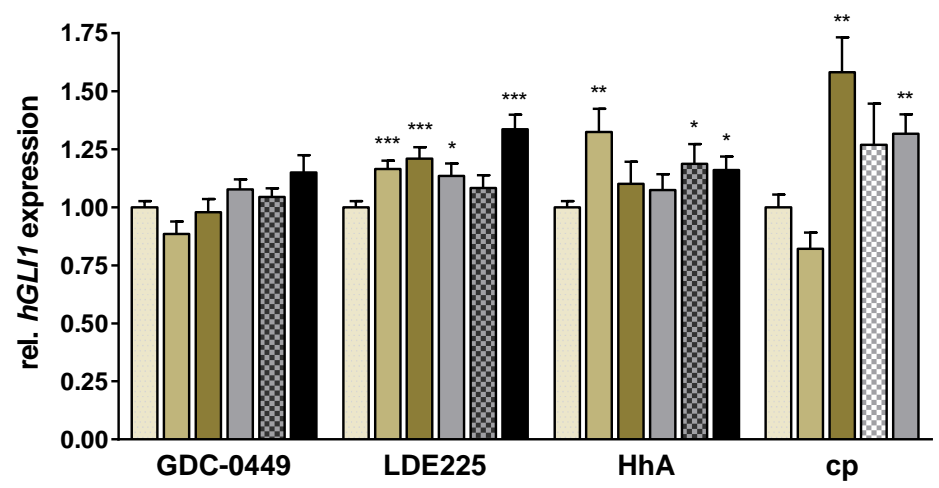

Rh41

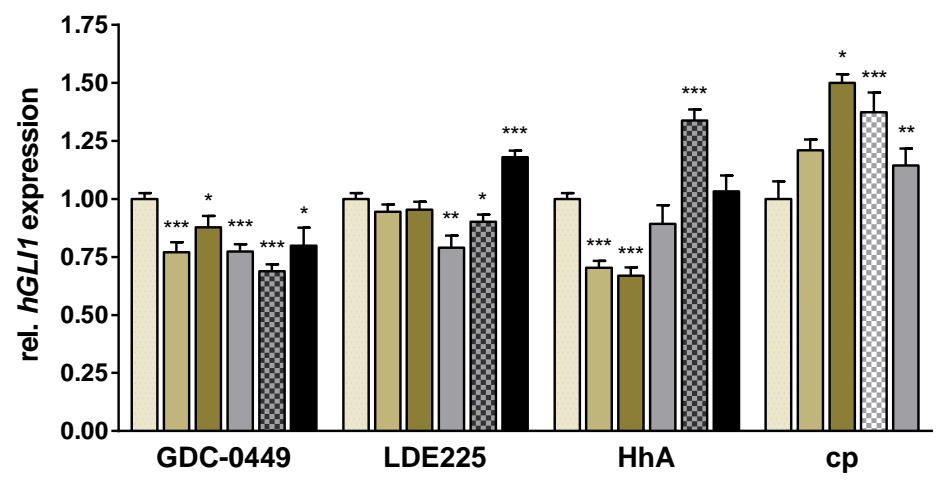

Figure 5: Modulation of HH signaling activity by SMO antagonists. Relative GLII expression in RD, RUCH-2, RMS-13 and Rh41 cells after incubation (24 h) with GDC-0449, LDE225, HhA and cyclopamine (cp) at the indicated concentrations. Gene expression was normalized to $18 S$ rRNA expression levels. The corresponding solvent controls (solv) were set to 1 . All data represent at least two independent experiments measured in triplicates. The data are displayed as mean $+/$-SEM; $*, \mathrm{p}<0.05 ; * *, \mathrm{p}<0.01 ; * * *, \mathrm{p}<0.001$. 


\section{RESULTS}

\subsubsection{Effects on $I G F 2$ expression}

Next, the effects of the drugs on IGF2 expression were analyzed because this gene plays an important role in the pathology of RMS (Martins et al., 2011) and is regulated by HH signaling in specific cellular settings (Corcoran et al., 2008; Hahn et al., 2000). RUCH-2 did not express $I G F 2$, whereas the remaining three cell lines did. As shown in Figure 6, all SMO antagonists modulated the expression levels of $I G F 2$, however to a variable extent.

In RD cells IGF2 expression was significantly decreased by $0.1,30$ and $50 \mu \mathrm{M}$ GDC-0449, by $50 \mu \mathrm{M}$ LDE225 as well as by 10,30 and $50 \mu \mathrm{M}$ HhA. Cyclopamine had no effect, whereas $0.1 \mu \mathrm{M}$ HhA slightly enhanced the IGF2 mRNA level (Figure 6). In RMS-13 cells IGF2 transcription was significantly blocked upon treatment with 0.1 and $1 \mu \mathrm{M}$ GDC-0449 and with $50 \mu \mathrm{M}$ LDE22 or HhA. In contrast, incubation with 0.1 and $1 \mu \mathrm{M}$ HhA as well as with $1-10 \mu \mathrm{M}$ cyclopamine resulted in a significant induction of IGF2 mRNA expression (Figure 6). A different situation was observed in Rh41 cells. In this cell line the IGF2 mRNA level was significantly inhibited only with $10 \mu \mathrm{M}$ cyclopamine, while all other drugs rather increased it. The induction of IGF2 expression was specifically obvious upon treatment with GDC-0449, which stimulated IGF2 expression at any concentration (Figure 6).

In summary, these data demonstrate that SMO inhibitors can regulate IGF2 expression in RMS cell lines. Similar to $G L I$ expression, the drugs exert different effects on $I G F 2$ transcription and the response of the RMS cell lines to the inhibitors is heterogeneous. Except for cyclopamine in RMS-13 cells, the modulation of IGF2 expression (Figure 6) is not necessarily correlated with the corresponding GLII expression (Figure 5). It rather seems that the modulation of GLII expression by SMO antagonists is independent from the regulation of IGF2. 

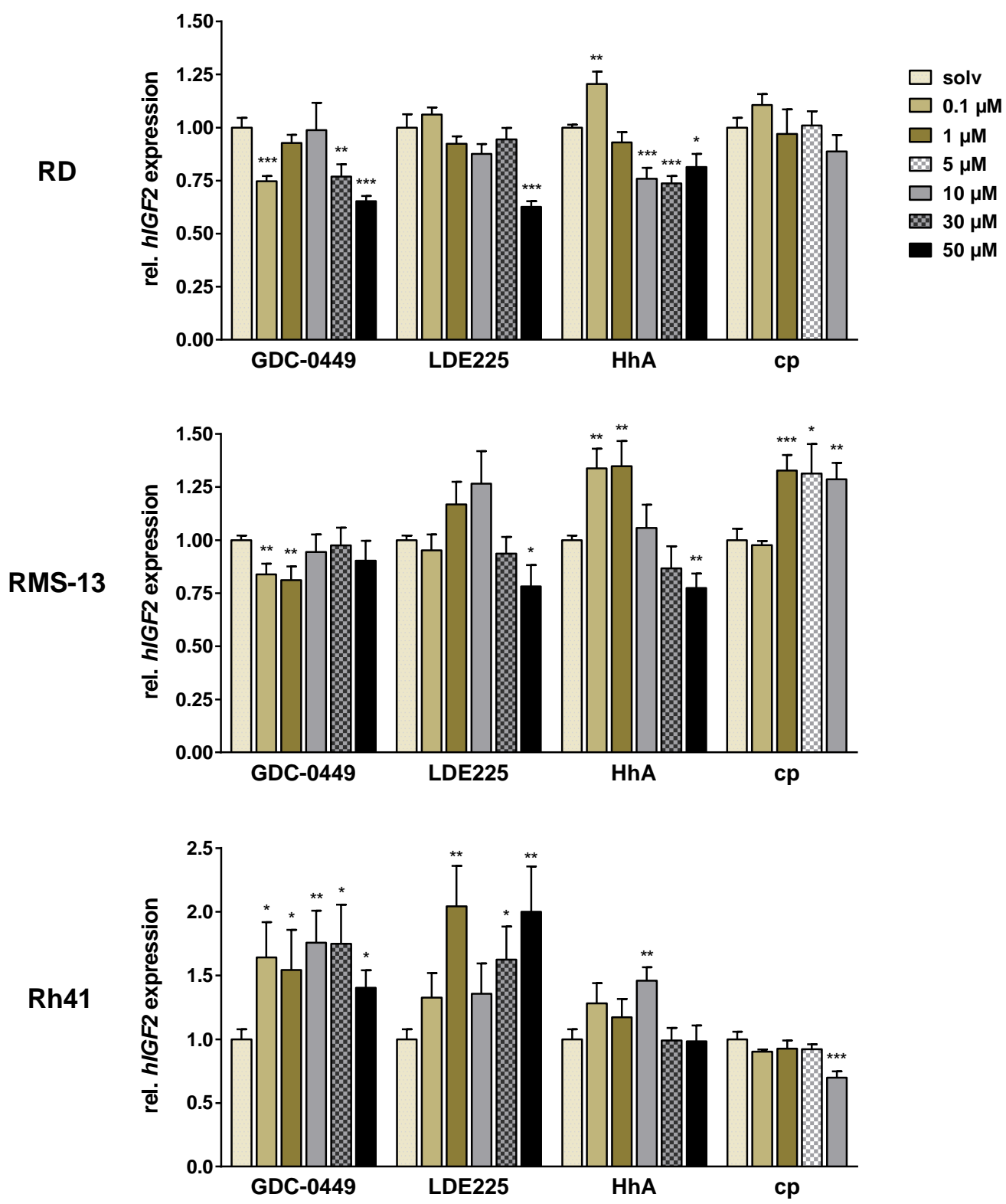

Figure 6: Modulation of IGF2 transcription by SMO antagonists. Relative IGF2 mRNA expression in RD, RMS-13 and Rh41 cells after incubation (24 h) with GDC-0449, LDE225, HhA and cyclopamine (cp) at the indicated concentrations. Gene expression was normalized to $18 \mathrm{~S}$ rRNA expression levels. The corresponding solvent controls (solv) were set to 1 . All data represent at least two independent experiments measured in triplicates. The data are presented as mean +/-SEM; *, $\mathrm{p}<0.05 ; * *, \mathrm{p}<0.01 ; * * *, \mathrm{p}<0.001$. 


\section{RESULTS}

\subsubsection{Effects on the expression of muscle differentiation markers}

In addition, the expression of the early and late muscle differentiation markers $M Y O D$ (Figure 7) and Myosin Heavy Chain 1 (MYH1) (Figure 8) respectively, were examined. While RUCH-2 cells neither expressed $M Y O D$ nor $M Y H 1$, modulation of the corresponding transcription levels was revealed in the three other RMS cell lines.

In RD cells $M Y O D$ mRNA expression was significantly decreased by 0.1 and $50 \mu \mathrm{M}$ GDC-0449, although $1 \mu \mathrm{M}$ GDC-0449 induced it (Figure 7). An inhibition of MYOD transcription was also observed for $0.1,10$ and $50 \mu \mathrm{M}$ LDE225, for 10,30 and $50 \mu \mathrm{M}$ HhA as well as for 0.1 and $1 \mu \mathrm{M}$ cyclopamine. In RMS-13 cells MYOD expression was blocked after treatment with 1 and $30 \mu \mathrm{M}$ GDC-0449, 30 and $50 \mu \mathrm{M}$ LDE225 or HhA and with 0.1 and $1 \mu \mathrm{M}$ cyclopamine (Figure 7). In contrast to RD and RMS-13, in Rh41 cells MYOD expression was induced by GDC-0449 and LDE225 at any concentration. MYOD transcription was also stimulated by 10,30 and $50 \mu \mathrm{M}$ HhA. However cyclopamine showed no effect on MYOD expression in this cell line (Figure 7).

MYH1 transcription in RD cells was significantly repressed by 30 and $50 \mu \mathrm{M}$ GDC-0449, by 10, 30 and $50 \mu \mathrm{M}$ LDE225 and by $1-50 \mu \mathrm{M}$ HhA (Figure 8). An induction of MYH1 expression in RD cells was revealed by cyclopamine at any concentration (Figure 8). In RMS-13 cells MYH1 expression was slightly induced by 0.1 and $30 \mu \mathrm{M}$ GDC-0449 and by 1-30 $\mu \mathrm{M}$ LDE225. HhA increased the expression of $\mathrm{MYHI}$ at a concentration of 0.1 and $1 \mu \mathrm{M}$, but decreased it at a concentration of 30 and $50 \mu \mathrm{M}$. Inhibition of $\mathrm{MYH1}$ transcription was also seen with $5 \mu \mathrm{M}$ cyclopamine (Figure 8). In Rh41 cells the dose response analyses showed an induction of MYH1 mRNA expression by $0.1,10$ and $30 \mu \mathrm{M}$ GDC-0449 and by 1 and $30 \mu \mathrm{M}$ LDE225, whereas $50 \mu \mathrm{M}$ LDE inhibited it. HhA activated the transcription of $M Y H 1$ at a concentration of 0.1 and $1 \mu \mathrm{M}$, but repressed it at a concentration of 30 and $50 \mu \mathrm{M}$. Treatment of cyclopamine resulted in an inhibition of $M Y H 1$ expression at 1-10 $\mu \mathrm{M}$ (Figure 8).

These findings show that inhibition of SMO by the four drugs is able to modulate the expression of the muscle differentiation markers $M Y O D$ and $M Y H 1$. However, the effects of the drugs are again heterogeneous and concentration dependent and differ from cell line to cell line. 
RD
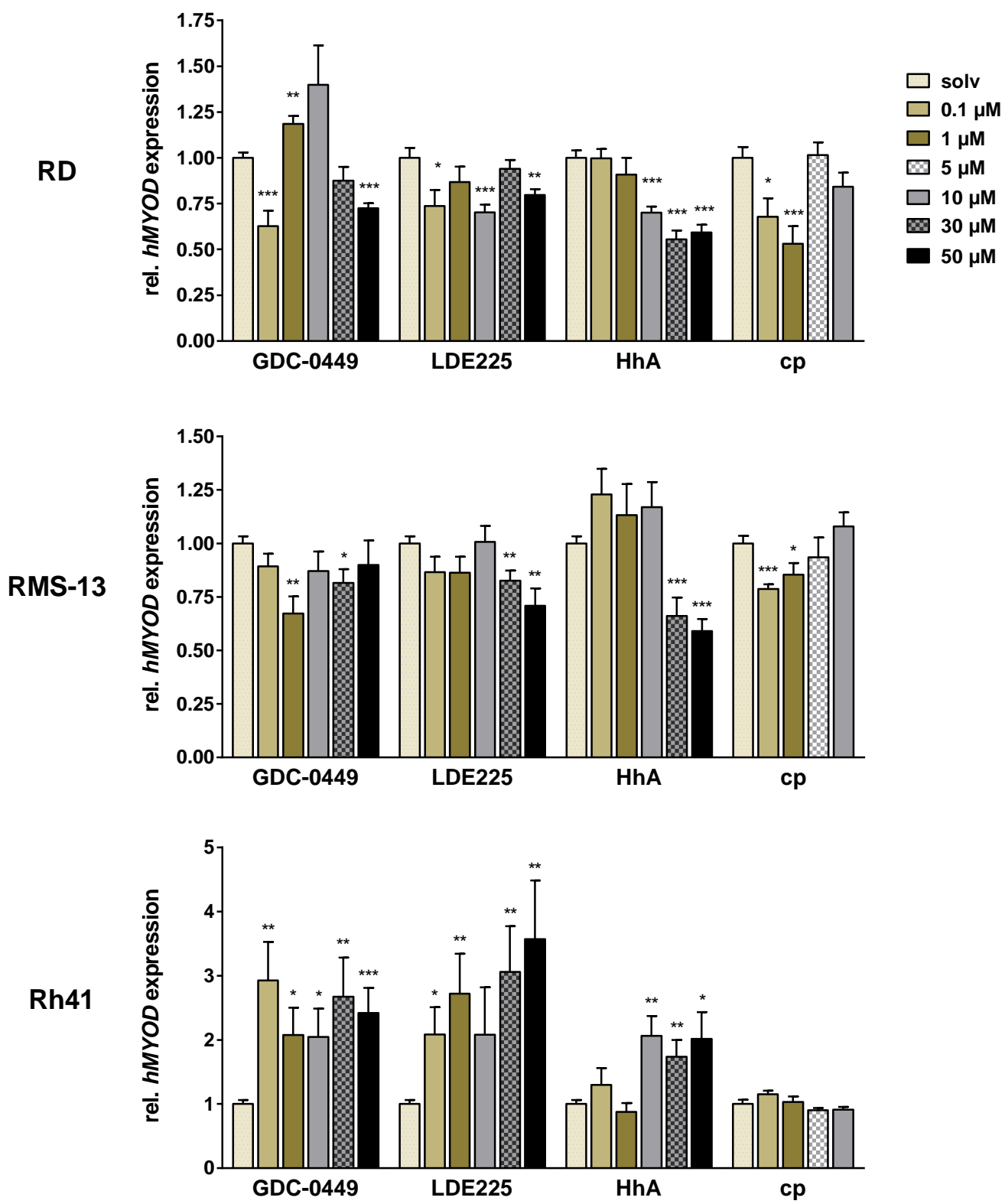

Figure 7: Modulation of MYOD transcription by SMO antagonists. Relative MYOD mRNA expression in RD, RMS-13 and Rh41 cells after incubation (24 h) with GDC-0449, LDE225, HhA and cyclopamine (cp) at the indicated concentrations. Gene expression was normalized to $18 S$ rRNA expression levels. The corresponding solvent controls (solv) were set to 1 . All data represent at least two independent experiments measured in triplicates. The data are presented as mean +/-SEM; *, $\mathrm{p}<0.05 ; * *, \mathrm{p}<0.01 ; * * *, \mathrm{p}<0.001$. 

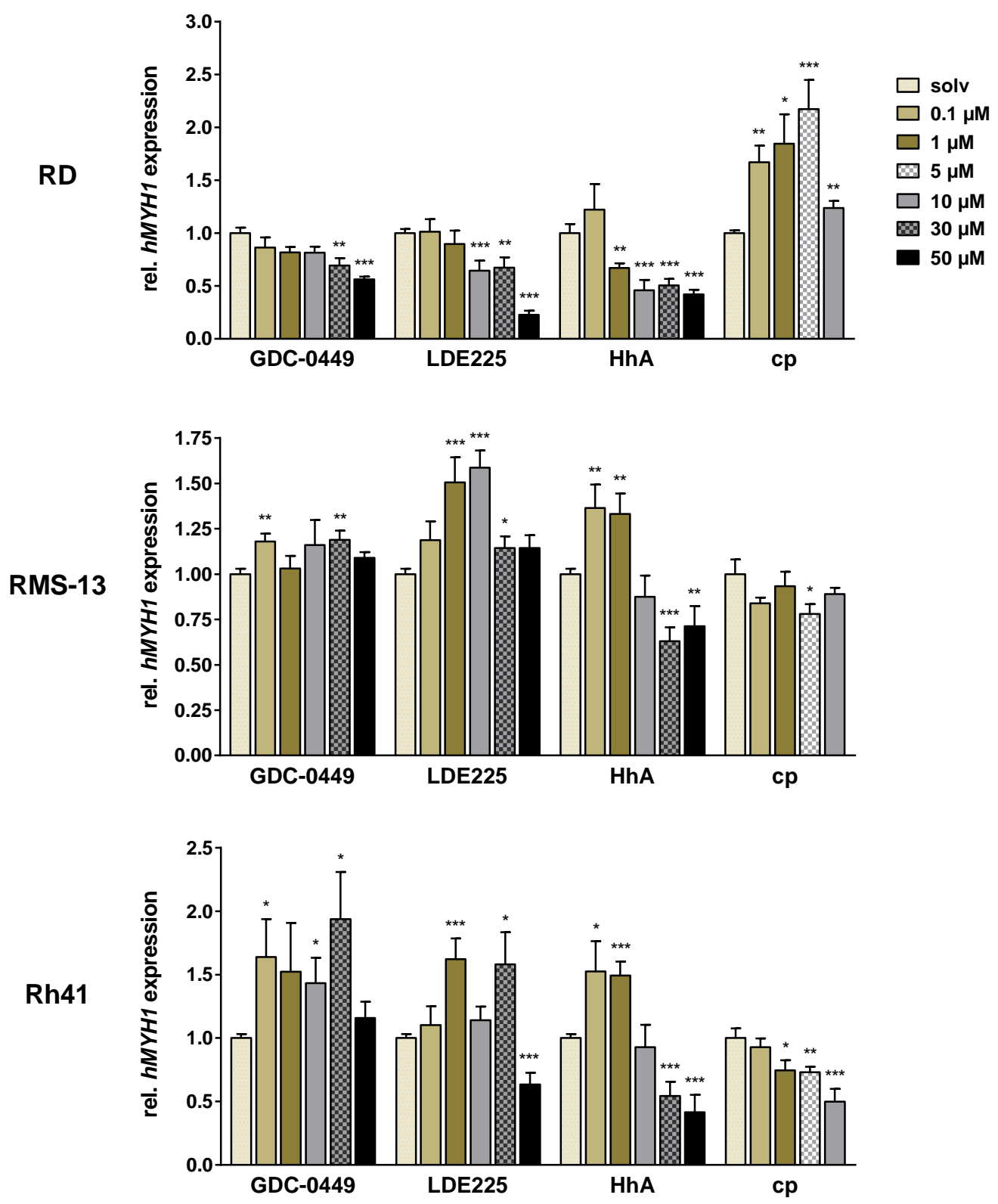

Figure 8: Modulation of $\mathbf{M Y H 1}$ transcription by SMO antagonists. Relative MYH1 mRNA expression in RD, RMS-13 and Rh41 cells after incubation (24 h) with GDC-0449, LDE225, HhA and cyclopamine (cp) at the indicated concentrations. Gene expression was normalized to $18 S$ rRNA expression levels. The corresponding solvent controls (solv) were set to 1 . All data represent at least two independent experiments measured in triplicates. The data are presented as mean $+/$-SEM; $*$, $\mathrm{p}<0.05 ; * *, \mathrm{p}<0.01 ; * * *, \mathrm{p}<0.001$. 


\subsubsection{Modulation of cellular viability, proliferation and apoptosis}

\subsubsection{Effects on cellular viability}

RD cells showed a triphasic change in GLII expression upon treatment with GDC-0449, LDE225 or HhA (i.e. a decrease at $10 \mu \mathrm{M}$, a re-increase at $30 \mu \mathrm{M}$ compared to $10 \mu \mathrm{M}$ and again a decrease at $50 \mu \mathrm{M}$; see Figure 5). In order to exclude that this was due to toxic effects of the drugs, cellular viability/metabolic activity was analyzed via WST assay.

As shown in Figure 9, treatment with different concentrations of SMO inhibitors was well tolerated by RD cells. GDC-0449 and cyclopamine did not reduce viability at any of the applied concentrations. A significant decrease in cellular viability was detected for $50 \mu \mathrm{M}$ LDE225 and $50 \mu \mathrm{M}$ HhA.

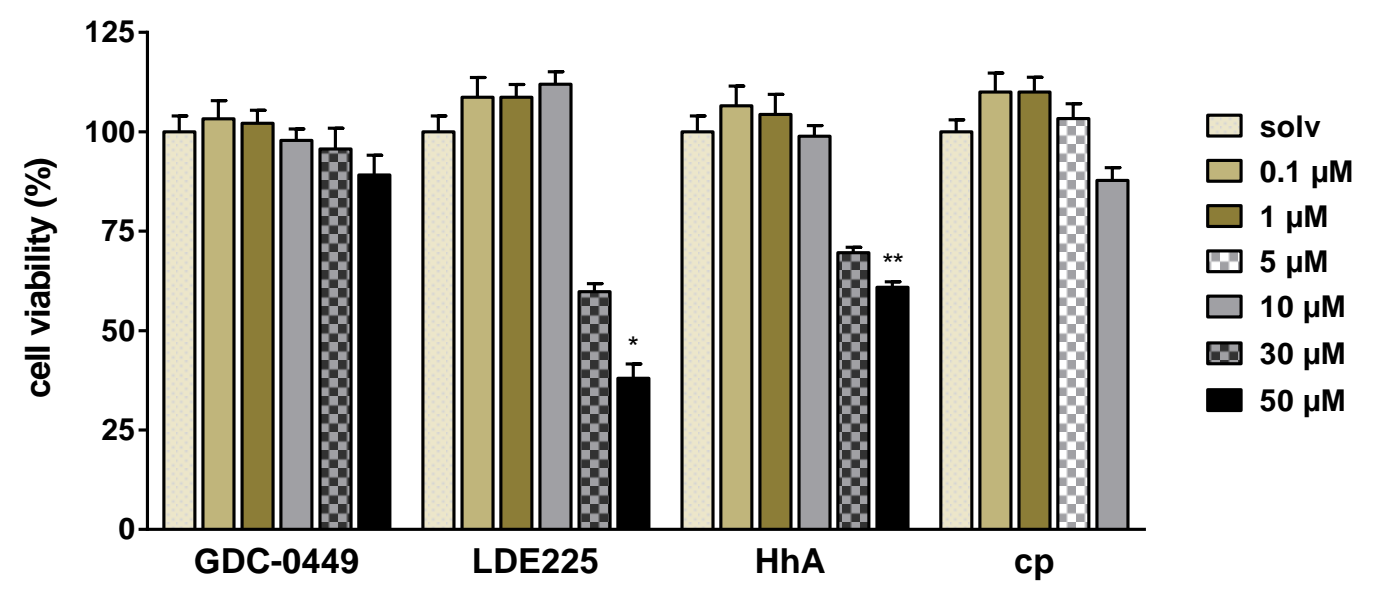

Figure 9: Cell viability/metabolic activity of RD cells after treatment with SMO antagonists. GDC-0449, LDE225, HhA and cyclopamine (cp) were incubated for $24 \mathrm{~h}$ with the cells at the indicated concentrations. Cellular viability was determined by WST assay. The respective solvent controls (solv) were set to $100 \%$. The data summarize two independent experiments and are represented as mean +/-SEM; *, p $<0.05 ; * *, p<0.01$.

Thus, RD cells showed no triphasic changes in cellular viability/metabolic activity upon treatment with GDC-0449, LDE225 or HhA. Instead, cellular viability continuously decreased with increasing doses of the drugs. In conclusion the re-increase of GLII expression at $30 \mu \mathrm{M}$ GDC-0449, LDE225 or HhA (compared to $10 \mu \mathrm{M}$ ) is not correlated with increased cell viability (compare Figure 5 with Figure 9). 


\section{RESULTS}

\subsubsection{Effects on cellular proliferation}

Next GDC-0449, LDE225, HhA, and cyclopamine were compared with respect to their potential to inhibit cellular proliferation. For this purpose concentrations were selected that either have reduced or did not impact on GLII expression in most experimental settings (10 $\mu \mathrm{M}$ and $30 \mu \mathrm{M}$, respectively). Cyclopamine was applied only at a concentration of $5 \mu \mathrm{M}$. BrdU incorporation into the DNA of the cells was measured as an indicator of proliferative activity.

In RD cells $30 \mu \mathrm{M}$ LDE225, 10 and $30 \mu \mathrm{M}$ HhA decreased the proliferation rate approximately by $55 \%, 30 \%$ and $50 \%$, respectively (Figure $10 \mathrm{~A}$ ). $30 \mu \mathrm{M}$ GDC-0449, $10 \mu \mathrm{M}$ LDE225 and cyclopamine did not show any antiproliferative effects, whereas $10 \mu \mathrm{M}$ GDC-0449 significantly increased the proliferation rate of the cells (Figure $10 \mathrm{~A}$ ). This was similar in RUCH-2 cells (Figure $10 \mathrm{~B}$ ), in which proliferation was significantly reduced by $30 \mu \mathrm{M}$ LDE225, 10 and $30 \mu \mathrm{M}$ HhA. As in RD cells $30 \mu \mathrm{M}$ GDC-0449 and $10 \mu \mathrm{M}$ LDE225 showed no impact on the proliferation rate, whereas $10 \mu \mathrm{M}$ GDC-0449 and $5 \mu \mathrm{M}$ cyclopamine significantly increased proliferation (by approximately $50 \%$ and $100 \%$, respectively; Figure 10 B). In RMS-13 cells antiproliferative effects were observed upon treatment with $30 \mu \mathrm{M}$ GDC-0449, 10 and $30 \mu \mathrm{M}$ LDE225 and by $30 \mu \mathrm{M}$ HhA (Figure $10 \mathrm{C}$ ). In Rh41 none of the SMO inhibitors reduced the proliferation rate (Figure $10 \mathrm{D}$ ). However, proliferation inducing effects were seen for $10 \mu \mathrm{M}$ and $30 \mu \mathrm{M}$ GDC-0449, as well as for $10 \mu \mathrm{M}$ LDE225 and for cyclopamine (Figure $10 \mathrm{D}$ ).

In summary, the BrdU incorporation assay reveals that the antiproliferative effects of SMO antagonists varies between the RMS cell lines. Apparently, LDE225 and HhA are the most potent inhibitors since antiproliferative effects are seen in many settings (e.g. $30 \mu \mathrm{M} \mathrm{HhA}$ reduced proliferation in RD, RUCH-2 and RMS-13 cells). Paradoxically, treatment with GDC-0449 or cyclopamine rather induces proliferation (particularly obvious for cyclopamine in RUCH-2 and Rh41 cells). 
A

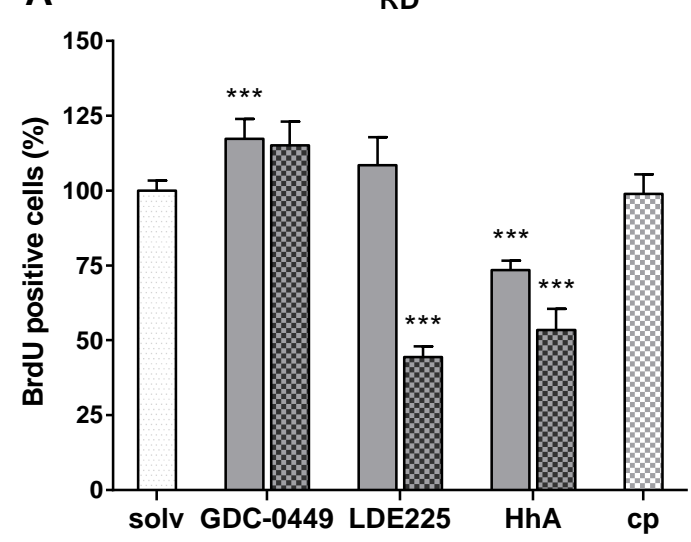

C

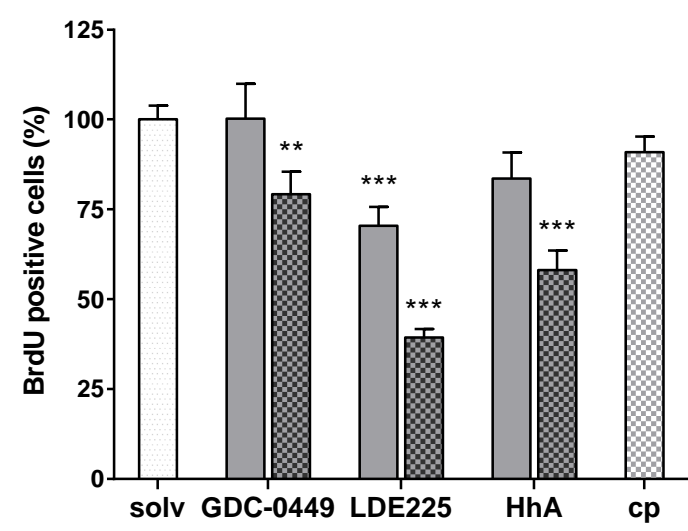

B

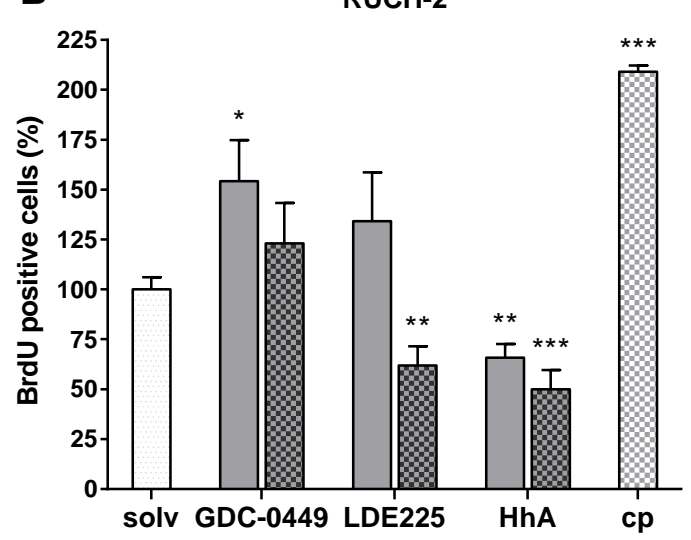

D

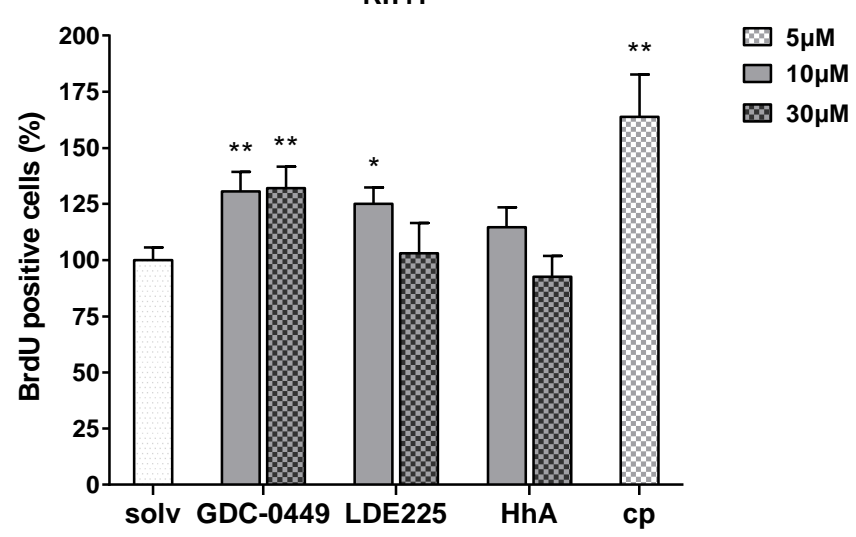

Figure 10: Modulation of proliferation by SMO antagonists. Measurement of BrdU incorporation in RD (A), RUCH-2 (B), RMS-13 (C) and Rh41(D) cells after incubation for $24 \mathrm{~h}$ with GDC-0449, LDE225, HhA and cyclopamine (cp) at the indicated concentrations. BrdU incorporation is shown as the percentage of the respective solvent controls (solv) that were set to $100 \%$. The data summarize three independent experiments and are represented as mean $+/$-SEM; $*$, $\mathrm{p}<0.05 ; * *, \mathrm{p}<0.01 ; * * *, \mathrm{p}<0.001$.

In addition to the BrdU incorporation analysis, proliferation was calculated by simple counting of RD and Rh41 cells (see 5.2.5) after treatment with SMO antagonists.

In RD cells a significant reduction of the cell number was revealed upon treatment with $30 \mu \mathrm{M}$ LDE225 and with $10 \mu \mathrm{M}$ and $30 \mu \mathrm{M}$ HhA (Figure $11 \mathrm{~A}$ ), thus confirming the results of the BrdU assay. In Rh41 cells treatment with $10 \mu \mathrm{M}$ HhA significantly decreased the amount of the cells (Figure $11 \mathrm{~B}$ ), whereas $10 \mu \mathrm{M}$ GDC-0449 and $10 \mu \mathrm{M}$ LDE225 increased the cell number. However, the latter increase was not significant compared to the control. Although the changes in cell numbers confirmed the BrdU data (compare Figure 10 with Figure 11), the effects were less pronounced or not significant, respectively. This difference might be caused by a delay in 
cell division. For instance Rh41 have a cell doubling time of $44 \mathrm{~h}$ (Kang et al., 2011), hence it is possible that at the time point of measurement $(24 \mathrm{~h})$ most cells had incorporated BrdU during S-phase, but have not divided yet.
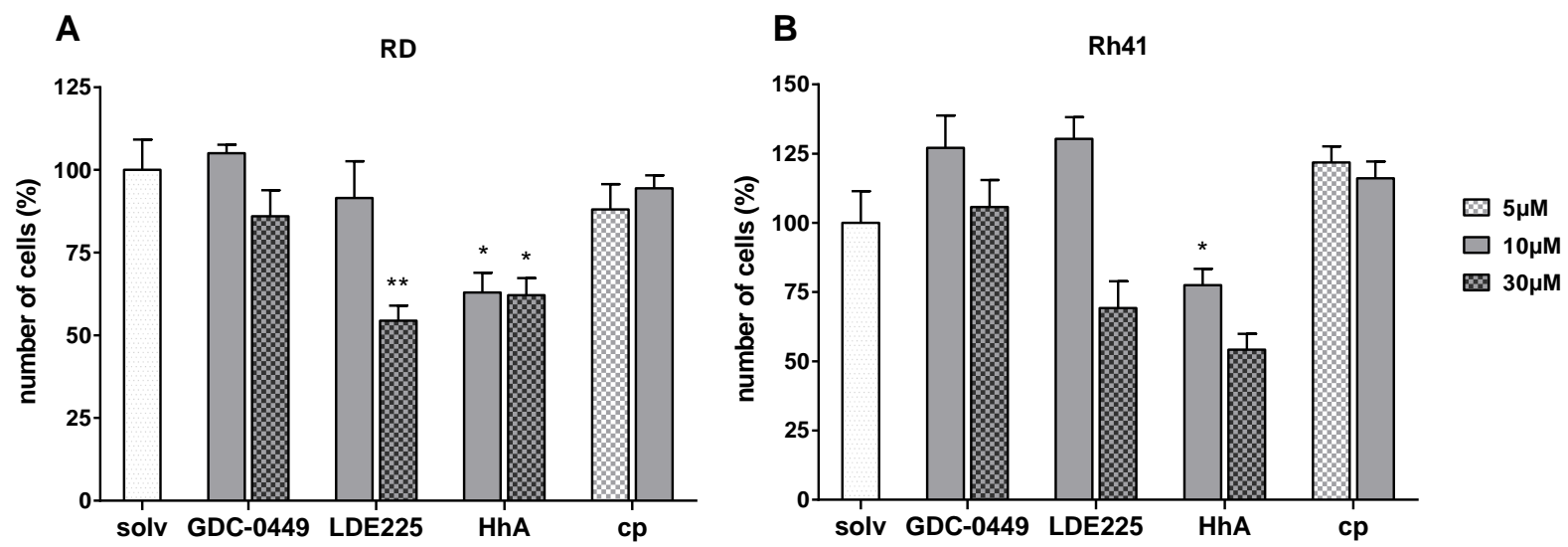

Figure 11: Determination of the cell numbers after treatment with SMO antagonists. Treatment was performed according to the experimental setup of the BrdU incorporation assay. $\mathrm{RD}$ (A) and $\mathrm{Rh} 41$ (B) cells were incubated for $24 \mathrm{~h}$ with GDC-0449, LDE225, HhA and cyclopamine (cp) at the indicated concentrations. Proliferation was determined by cell counting. The respective solvent controls (solv) were set to $100 \%$. The data summarize three independent experiments and are represented as mean $+/$-SEM; $*$, $\mathrm{p}<0.05 ; * *, \mathrm{p}<0.01$.

\subsubsection{Effects on apoptosis}

Next, the effects of GDC-0449, LDE225, HhA, and cyclopamine were compared concerning their potential to induce apoptosis. In order to measure apoptosis, a flow cytometry based assay with AnnexinV-FITC and PI was performed and the apoptosis rate was calculated by the amount of AnnexinV positive cells. For this purpose early-apoptotic (AnnexinV positive/PI negative) and late-apoptotic (AnnexinV positive/PI positive) cells were summed up (see 5.2.7). Analysis of apoptosis in RUCH-2 cells was not possible because of highly unstable results obtained from three independent measurements.

In RD cells (Figure $12 \mathrm{~A}) 30 \mu \mathrm{M}$ LDE225 and $30 \mu \mathrm{M}$ HhA significantly increased the apoptosis rate. $10 \mu \mathrm{M}$ LDE225, $10 \mu \mathrm{M}$ HhA as well as GDC-0449 and cyclopamine did not trigger apoptosis. The SMO inhibitors had similar effects in RMS-13 (Figure $12 \mathrm{~B}$ ). Besides a strong induction of apoptosis upon treatment with $30 \mu \mathrm{M}$ LDE225 and $30 \mu \mathrm{M} \mathrm{HhA}$, the apoptosis rate was also significantly increased using $10 \mu \mathrm{M}$ HhA and $30 \mu \mathrm{M}$ GDC-0449, whereas cyclopamine had no effect. In Rh41 cells (Figure $12 \mathrm{C}$ ) the SMO inhibitors induced comparable proapoptotic 
effects as in RMS-13 cells. In addition, a slight but significant induction of apoptosis was also caused by $10 \mu \mathrm{M}$ GDC-0449.

In summary, the results show that $30 \mu \mathrm{M}$ LDE225 and HhA exert proapoptotic effects in all three RMS cell lines, whereas low doses of HhA increases apoptosis in RMS-13 and Rh41 and $10 \mu \mathrm{M}$ LDE225 does not induce apoptosis at all. In contrast, 10 and $30 \mu \mathrm{M}$ GDC-0449 only marginally stimulate apoptosis in RMS-13 and Rh41, and cyclopamine is not able to induce proapoptotic effects in any of the examined RMS cell lines.

A

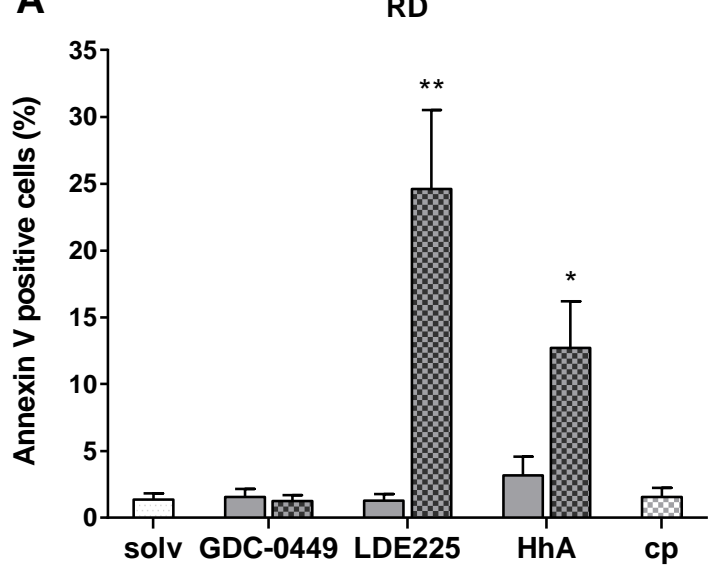

C

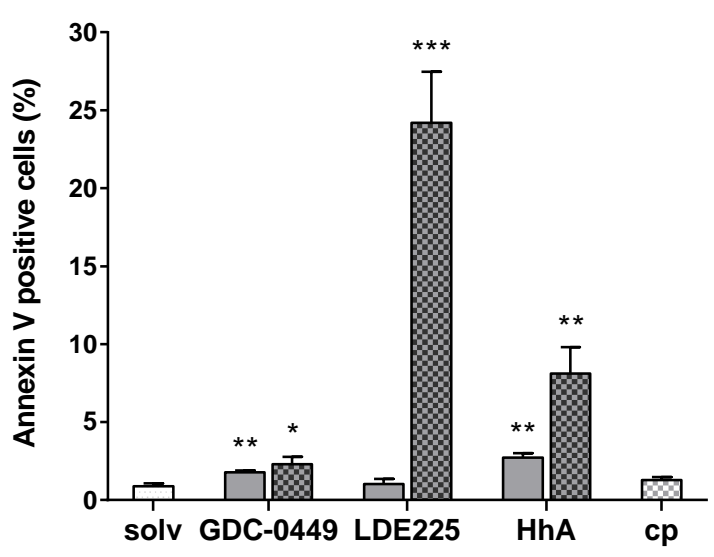

B

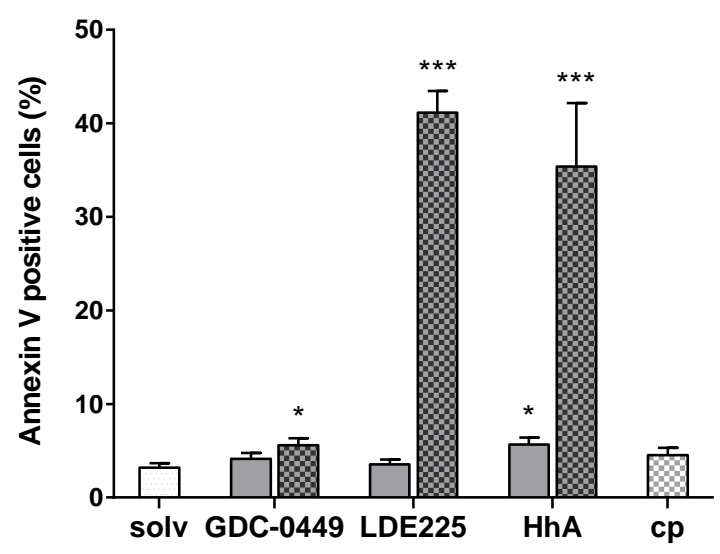

$\square 5 \mu \mathrm{M}$
$\square 10 \mu \mathrm{M}$
$\square 30 \mu \mathrm{M}$

Figure 12: Modulation of apoptosis by SMO antagonists. RD (A), RMS-13 (B) and Rh41 (C) cells were incubated for $48 \mathrm{~h}$ with GDC-0449, LDE225, HhA and cyclopamine (cp) at the indicated concentrations. Apoptotic cells are illustrated as AnnexinV positive cells in percent. All data represent three independent experiments measured in duplicates and were displayed as mean +/-SEM; solv, solvent; *, $\mathrm{p}<0.05 ; * *, \mathrm{p}<0.01 ; * * *, \mathrm{p}<0.001$. 


\section{RESULTS}

In conclusion, these data demonstrate that SMO inhibitors differ remarkably in their potential to modulate cellular proliferation and to induce apoptosis in the RMS cell lines. Thus, cyclopamine neither reduces the proliferation rate nor induces apoptosis in the used cell lines. It rather enhances the proliferation rate of RUCH-2 and Rh41 cells (Figure 10 B, D). Moreover, GDC-0449 is less effective compared to LDE225 or HhA, which have the capacity to trigger antiproliferative effects and to induce apoptosis that is dependent on the cell line and the applied drug concentration.

\subsubsection{Effects on PI3K/AKT/mTOR signaling, on AMPK activity and on LC3-II expression}

It is known that $\mathrm{HH}$ signaling can influence the activation status of PI3K/AKT signaling ( $\mathrm{Fu}$ et al., 2006; Morton et al., 2007; Riobo et al., 2006), the activity of mTOR(Wang et al., 2012) and can inhibit autophagy (Jimenez-Sanchez et al., 2012). Moreover, HH inhibitors can impact on a noncanonical SMO/Ca ${ }^{2+} / \mathrm{AMPK}$ dependent signaling cascade (Teperino et al., 2012). Due to these data, the impact of the four SMO inhibitors on the phosphorylation status of AKT and AMPK was investigated. Analysis of the phosphorylation status of S6 served as surrogate readout for mTOR activity. For analysis of caspase 3 activity an antibody was used, that detects the inactive preform ( $35 \mathrm{kDa})$ of caspase 3 as well as its activated cleavage products (19 and 17 $\mathrm{kDa})$. Autophagy was examined by the expression levels of the cytosolic LC3-I (16 kDa) and the LC3-II (14 kDa). LC3-II is the lipidated form of LC3-I and specifically associates with autophagosome membranes and thus reflects autophagosome numbers (Mizushima et al., 2010). In the following the most important and clear-cut results from at least two independent western blot analyses are summarized.

In RD cells, GDC-0449, LDE225 and cyclopamine did not modulate the phosphorylation status of AKT or S6 in comparison to the solvent (Figure 13 A). In contrast, incubation with HhA decreased AKT/pAKT levels, whereas cyclopamine rather increased it. An induction of AMPK phosphorylation was observed with $30 \mu \mathrm{M}$ GDC-0449 and with 10 and $30 \mu \mathrm{M}$ LDE225. Activity of caspase 3 was induced by $30 \mu \mathrm{M}$ LDE225 (indicated by an increase of cleaved caspase 3 levels) as well as autophagy. Increased LC3-II levels were also seen by treatment with HhA and cyclopamine at any concentration (Figure 13 A). In RUCH-2 cells and similar to RD cells, HhA reduced AKT/pAKT levels, $30 \mu \mathrm{M}$ LDE225 induced caspase 3 activity and autophagy (Figure 13 B). Additionally, $30 \mu \mathrm{M}$ LDE225 reduced pAMPK phosphorylation. An increase in LC3-II expression was detected with HhA and cyclopamine. In RMS-13 cells clear-cut effects were 
found for $30 \mu \mathrm{M}$ LDE225 on caspase 3 activation and on LC3-II levels (Figure $13 \mathrm{C}$ ). LC3-II was also increased by $30 \mu \mathrm{M} \mathrm{HhA}$ and by cyclopamine at any concentration. In Rh41 cells GDC-0449 and HhA did not exert any obvious effects (Figure 13 D). Activation of caspase 3 and increased LC3-II levels were detected upon treatment with $30 \mu \mathrm{M}$ LDE225. An increase in LC3-II was also seen after treatment of Rh41 cells with cyclopamine.
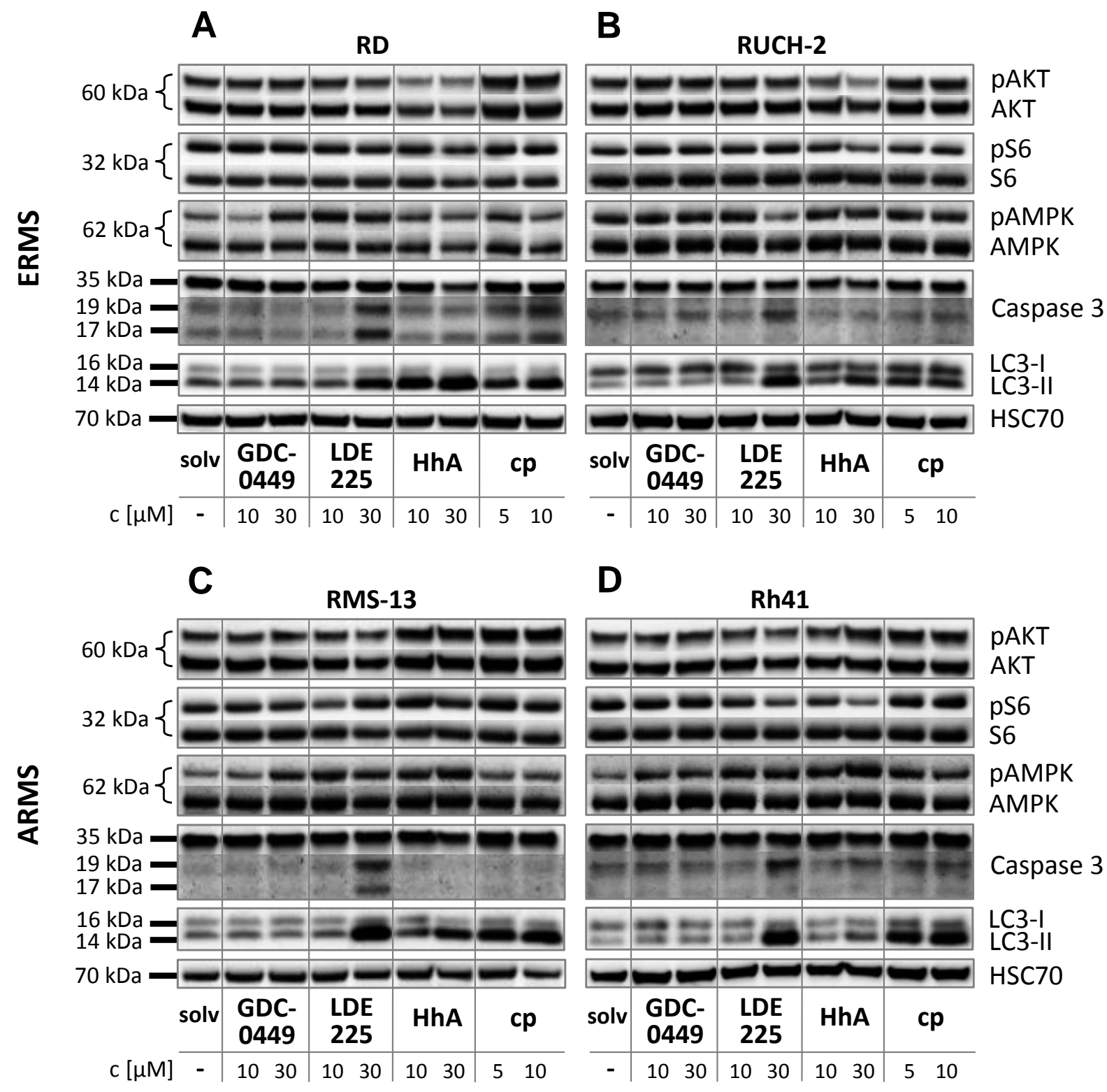

D

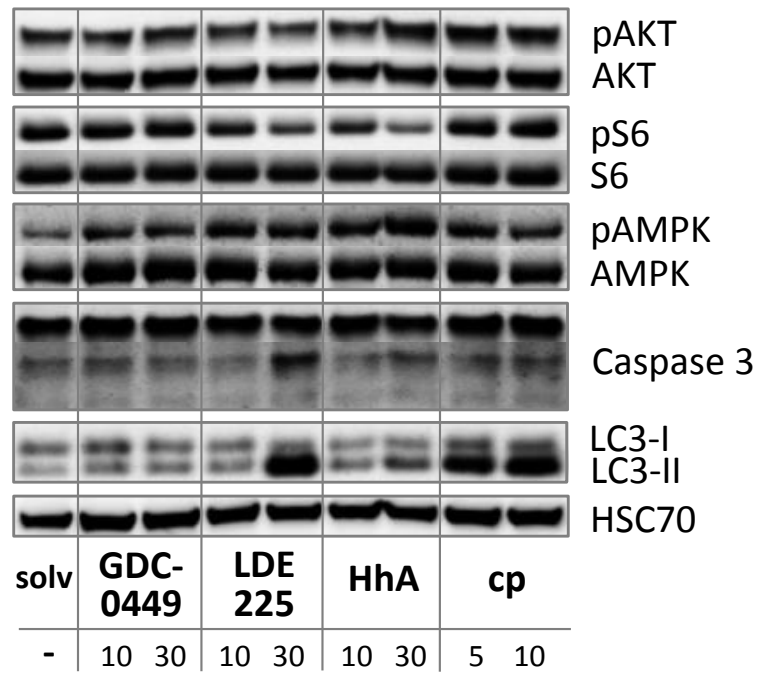

Figure 13: Impact of SMO antagonists on PI3K/AKT/mTOR signaling, phosphorylation of AMPK and LC3-II protein levels. Western blot analysis of RD (A), RUCH-2 (B), RMS-13 (C) and Rh41 (D) cells after incubation for $48 \mathrm{~h}$ with GDC-0449, LDE225, HhA and cyclopamine (cp) at the indicated concentrations. HSC70 expression levels served as loading control. Representative blots of two independent experiments are shown. solv, solvent. 


\section{RESULTS}

Again, these data demonstrate that SMO inhibitors exert highly diverse effects on the above mentioned parameters and that the response of the cell lines is very heterogeneous. Whereas GDC-0449 hardly triggered any effects, $30 \mu \mathrm{M}$ LDE225 activated caspase 3 and autophagy in all cell lines and cyclopamine usually induced pAKT and activated autophagy. HhA inhibited pAKT expression in ERMS cells and increased LC3-II levels (Figure 13 A, B), whereas these

effects are variable in ARMS cells (Figure $13 \mathrm{C}$, D). Interestingly, HhA did not stimulate caspase 3 activation in any of the RMS cell lines although AnnexinV positive cells were increased upon HhA treatment (Figure 12). This may indicate a caspase 3 independent apoptosis mechanism for HhA.

In addition to the western blot analyses of human RMS cell lines, the effects of SMO antagonists were also investigated in murine primary RMS cells, which were isolated from RMS bearing $\mathrm{Ptch}^{+/-}$mice (as described in chapter 5.2.3) and incubated with $10 \mu \mathrm{M}$ of each drug for $24 \mathrm{~h}$.

Similarly to human RMS cell lines, GDC-0449 did not impact on PI3K/Akt/mTOR signaling or Lc3-II expression (Figure 14). Treatment with LDE225 induced pAmpk/Ampk, comparable to the findings in RD cells (Figure 13 A). Analogue to human ERMS cells, HhA reduced pAkt/Akt and induced Lc3-II (compare Figure 13 A, B with Figure 14). HhA additionally decreased the protein level of Igf2. Furthermore and corresponding to the analyzed human cell lines (Figure 13), cyclopamine stimulated autophagy in primary murine RMS cells (Figure 14).

Together these data demonstrate that SMO inhibitors also exert heterogeneous effects in primary murine RMS cells. 


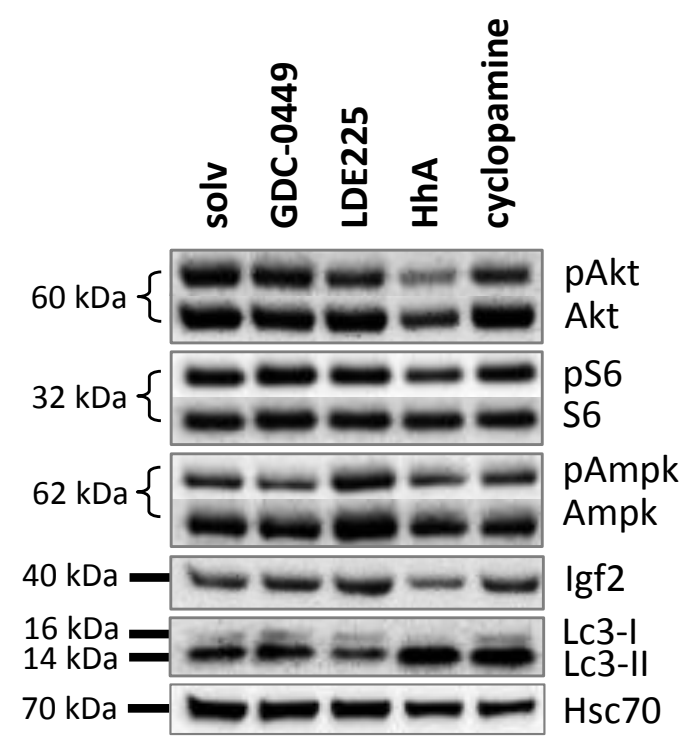

Figure 14: Impact of SMO antagonists on PI3K/Akt/mTOR signaling, phosphorylation of Ampk and Lc3-II protein levels in murine primary RMS cells. Isolated primary RMS cells were incubated for $24 \mathrm{~h}$ with $10 \mu \mathrm{M}$ GDC-0449, LDE225, HhA or cyclopamine (cp) and analyzed by western blot. Hsc70 expression levels served as loading control. The data represent the results from one experiment. solv, solvent.

The findings that HhA reduced phosphorylation of AKT in the human ERMS cell lines RD and RUCH-2 (Figure 13 A, B) as well as in murine primary RMS cells (Figure 14) suggests that SMO potentially regulates AKT phosphorylation in ERMS subtypes. In order to verify the speculation that SMO might control AKT phosphorylation, RD cells were incubated with the SMO agonist (SAG).

As revealed by western blot analysis phosphorylation of AKT was increased by $100 \mathrm{nM}$ and $1 \mu \mathrm{M}$ SAG (Figure $15 \mathrm{~A}$ ), which was suppressed when the cells were concomitantly incubated with HhA. This indicates that HhA indeed regulates AKT phosphorylation at the level of SMO. Phosphorylation of S6 was marginally reduced after treatment with $10 \mu \mathrm{M}$ HhA plus $1 \mu \mathrm{M}$ SAG. IGF2 was slightly reduced by $10 \mu \mathrm{M}$ HhA, but its expression was strongly stimulated by $100 \mathrm{nM}$ SAG, verifying that IGF2 is a downstream target of $\mathrm{HH}$ signaling. Combination of HhA and SAG resulted in a decrease in IGF2 levels compared to the control at any concentration. Induction of autophagy was observed after incubation with HhA and also with $1 \mu \mathrm{M} \mathrm{SAG}$. The latter drugs apparently cooperated in LC3-II induction as demonstrated when both were combined (10 $\mu \mathrm{M}$ HhA plus $1 \mu \mathrm{M}$ SAG, Figure $15 \mathrm{~A})$. There were no detectable differences in pAMPK/AMPK and caspase 3 protein expressions (data not shown). Simultaneously, the effect 
of SAG on HH pathway activation was analyzed via GLI1 specific qRT-PCR (Figure $15 \mathrm{~B}$ ). However, SAG did not stimulate HH signaling activity at any concentration.

Together, these findings indicate that SMO might be involved in pAKT regulation, although this seems to be independent of GLII expression. However, these results are preliminary and the respective experiments have to be repeated in the future.

A
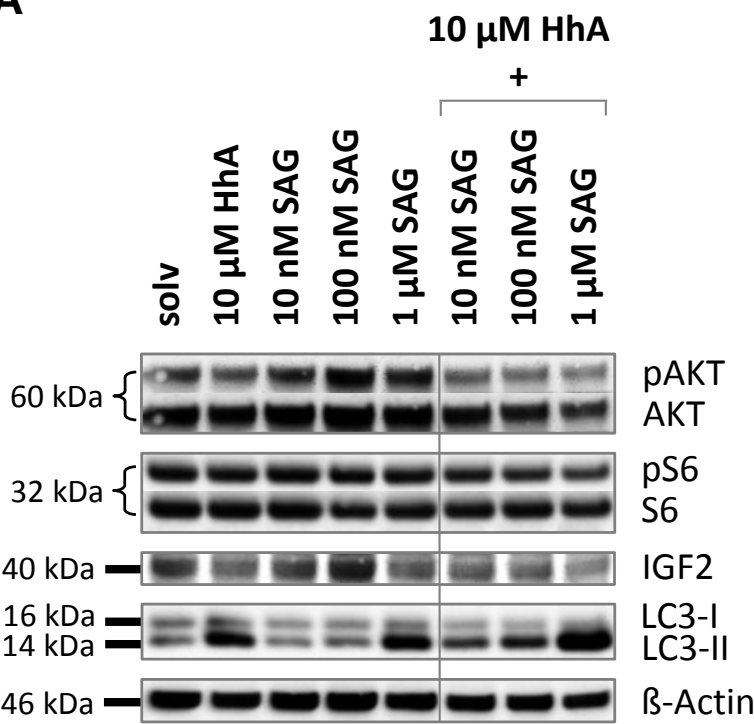

B

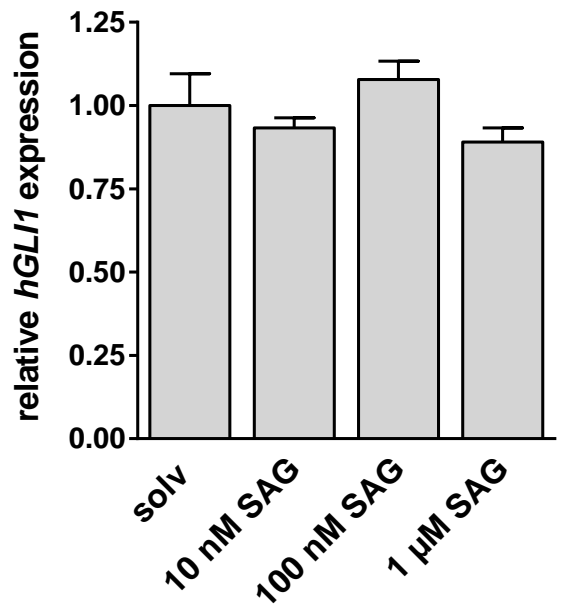

Figure 15: Effects of SMO activation by SAG on PI3K/AKT/mTOR signaling, IGF2 and autophagy as well as on HH signaling activity. (A) Western blot analysis of RD cells after incubation for $48 \mathrm{~h}$ with $\mathrm{HhA}$ and/or SAG at the indicated concentrations. B-Actin expression levels served as loading control. (B) Relative GLII expression in $\mathrm{RD}$ cells after incubation $(24 \mathrm{~h})$ with SAG at the indicated concentrations. Gene expression was measured in triplicates and normalized to $18 S$ rRNA expression. The corresponding solvent control (solv) was set to 1 and the data is displayed as mean +/-SEM and represent the results from one experiment. 


\subsection{Effects of SMO antagonists in combination with PI3K/AKT/mTOR inhibitors on RMS cell lines}

The second part of this thesis focused on the combination of SMO antagonists with inhibitors of the $\mathrm{PI} 3 \mathrm{~K} / \mathrm{AKT} / \mathrm{mTOR}$ pathway in order to improve potential anticancer effects.

Indeed, the combination of the dual PI3K/mTOR inhibitor PI103 with DOX, that is applied in the therapy of RMS, significantly enhanced apoptosis in RD, RMS-13 and Rh41 cells when compared to single drug treatments (Figure 16).

RD

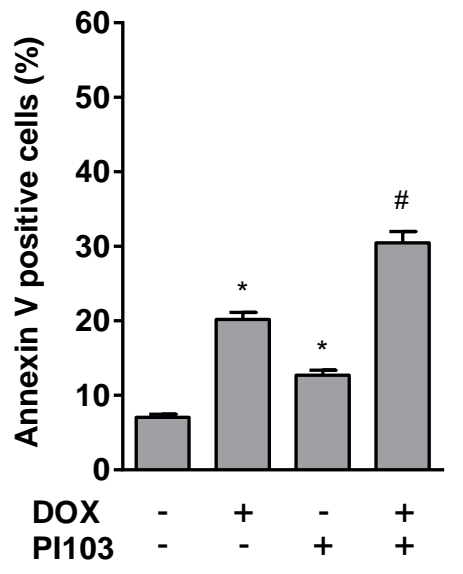

RMS-13

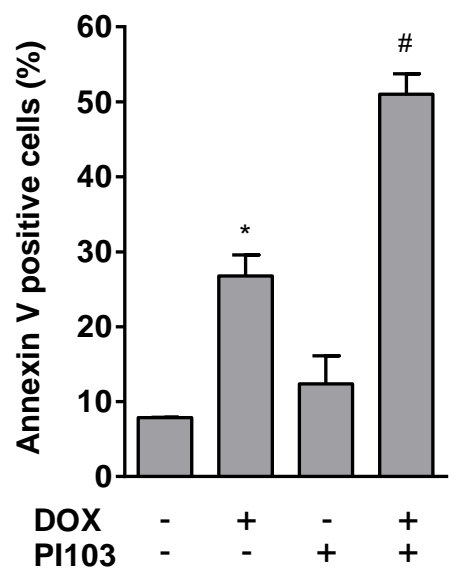

Rh41

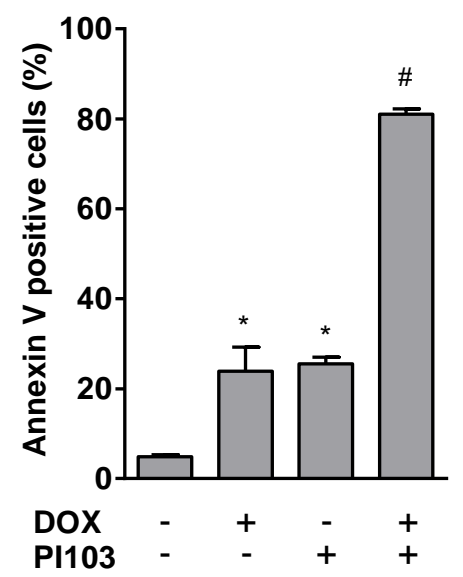

Figure 16: Cooperative effects on apoptosis upon treatment with a PI3K/mTOR inhibitor plus DOX. RD, RMS-13 and Rh41 cells were incubated for $24 \mathrm{~h}$ with $1 \mu \mathrm{M}$ DOX and/or $3 \mu \mathrm{M}$ PI103. Apoptotic cells are illustrated as AnnexinV positive cells in percent. The data represent two independent experiments for RD and one experiment each for RMS-13 and Rh41 and are displayed as mean +/-SEM; *, p<0.05; compared to solvent; \#, p $<0.05$ compared to cells treated with either drug alone. Comparisons were made with ANOVA/Tukey's testing.

Because i) PI3K/AKT/mTOR inhibition has antitumoral effects by itself (e.g. induced apoptosis in several RMS cells lines; Figure 16), ii) PI3K/AKT/mTOR inhibitors cooperate with other drugs in the induction of antitumoral effects (i.e. PI103 cooperated with DOX in induction of apoptosis; Figure 16) and iii) PI3K/AKT/mTOR signaling cooperates with $\mathrm{HH}$ signaling in tumor formation (see 2.7), SMO antagonists were combined with PI3K/AKT/mTOR inhibitors and the effects on the respective signaling pathways, proliferation, apoptosis, muscle differentiation, on AMPK activation and on autophagy were investigated. In order to block $\mathrm{PI} 3 \mathrm{~K} / \mathrm{AKT} / \mathrm{mTOR}$ signaling, the dual PI3K/mTOR inhibitor PI103, the specific PI3K inhibitor 


\section{RESULTS}

GDC-0941, the pure AKT inhibitor MK-2206 and the mTOR inhibitors everolimus and rapamycin were applied (see Figure 4).

\subsubsection{Proapoptotic effects in RD cells}

Initially, it was analyzed whether potential cooperative antitumor effects of PI3K/AKT/mTOR inhibitors plus SMO inhibitors are influenced by the concentration of the latter drugs. For that purpose, low $(10 \mu \mathrm{M})$ and high $(30 \mu \mathrm{M})$ concentrations of the corresponding SMO antagonists were applied and apoptosis was measured by FACS exemplarily in the ERMS cell line RD.

A significant induction of apoptosis was revealed after treatment with PI103 and GDC-0941 (approximately up to $12 \%$ and $8 \%$ respectively), whereas MK-2206, everolimus and rapamycin had no effect (Figure 17).

As described before (see Figure $12 \mathrm{~A}$ ) neither $10 \mu \mathrm{M}$ nor $30 \mu \mathrm{M}$ GDC-0449 stimulated apoptosis (Figure 17 A). The combination of $10 \mu \mathrm{M}$ GDC-0449 plus GDC-0941 or rapamycin significantly strengthened the apoptosis rate compared to the single drug treatments. Cooperative proapoptotic effects were also observed for $30 \mu$ M GDC-0449 plus MK-2206 or rapamycin (Figure 17 A).

Treatment with $10 \mu \mathrm{M}$ LDE225 did not trigger apoptosis, whereas $30 \mu \mathrm{M}$ strongly induced it compared to solvent control (Figure $17 \mathrm{~B}$ ). Cooperative proapoptotic effects were observed for $30 \mu \mathrm{M}$ LDE225 plus PI103 or plus GDC-0941.

As already shown in Figure 12 A, $30 \mu \mathrm{M}$ HhA increased the number of AnnexinV positive cells (Figure $17 \mathrm{C}$ ). Cooperative proapoptotic effects were revealed for the combination of $10 \mu \mathrm{M}$ HhA plus PI103 and GDC-0941, whereas an enormous induction of apoptosis was achieved by $10 \mu \mathrm{M}$ or $30 \mu \mathrm{M}$ HhA plus MK-2206 (up to $45 \%$ and $68 \%$, respectively).

Single treatment with $10 \mu \mathrm{M}$ cyclopamine only marginally induced apoptosis (Figure $17 \mathrm{D}$ ). Cooperative proapoptotic effects were detected for 5 and $10 \mu \mathrm{M}$ cyclopamine plus GDC-0941 or MK-2206.

Together, the data demonstrate that SMO antagonists in combination with PI3K/AKT/mTOR inhibitors cause different effects on apoptosis in RD cells. In the following experiments $10 \mu \mathrm{M}$ concentrations of GDC-0449, LDE225 and HhA were used. This was done due to the fact that $10 \mu \mathrm{M}$ GDC-0449, $10 \mu \mathrm{M}$ HhA or $5 \mu \mathrm{M}$ cyclopamine were in generally as potent as the respective higher concentration (exception was $30 \mu \mathrm{M}$ HhA plus MK-2206) and that the apoptosis induction using $30 \mu \mathrm{M}$ LDE225 was extremely high (i.e. at that concentration potential combination effects could have been overlooked). 


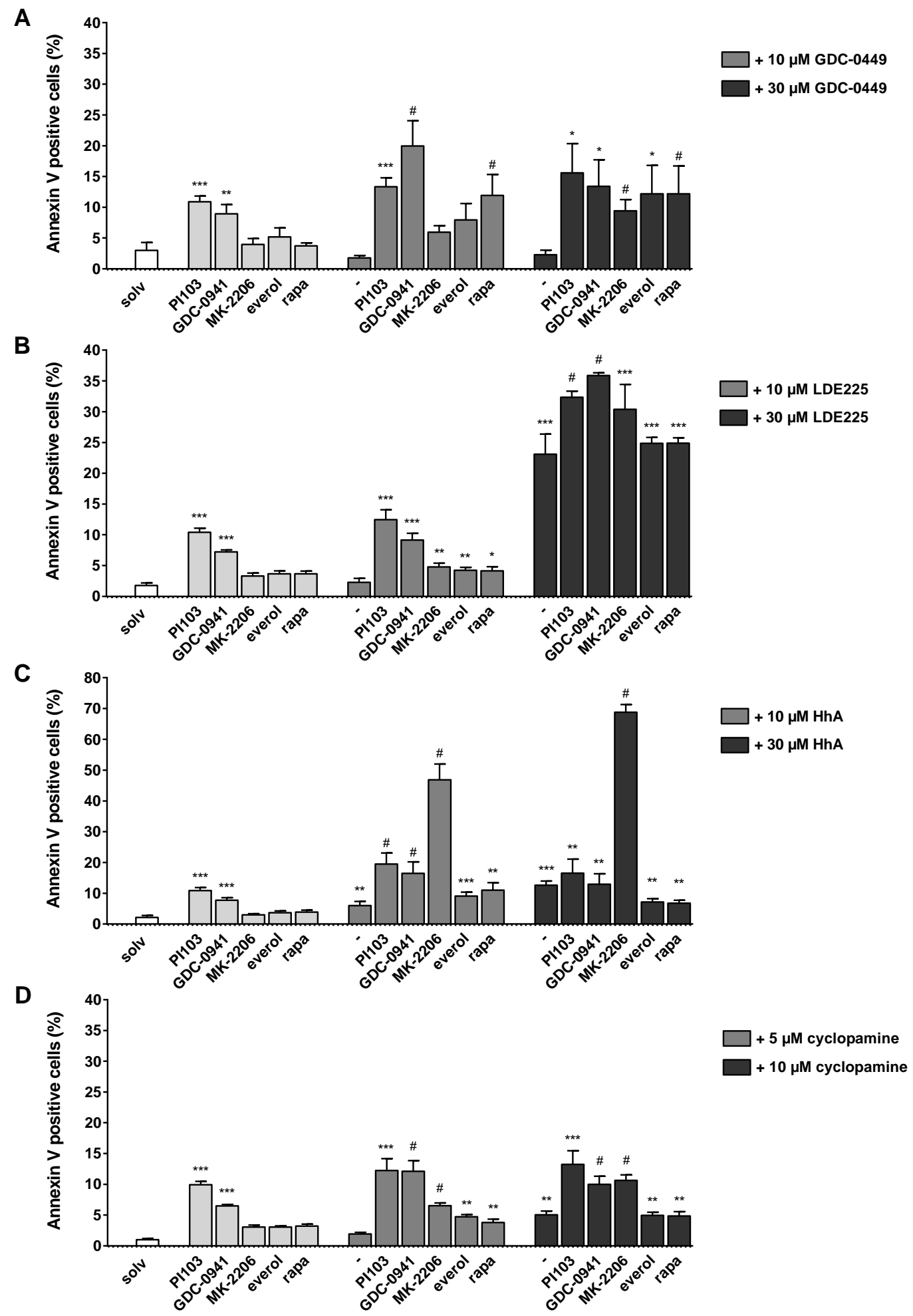

Figure 17: Regulation of apoptosis by SMO antagonists and/or PI3K/AKT/mTOR inhibitors. RD cells were incubated (48 h) with $10 \mu \mathrm{M}$ or $30 \mu \mathrm{M}$ GDC-0449 (A), LDE225 (B), HhA (C), $5 \mu \mathrm{M}$ or $10 \mu \mathrm{M}$ cyclopamine (D) and/or $3 \mu \mathrm{M}$ PI103, $10 \mu \mathrm{M}$ GDC-0941, $5 \mu \mathrm{M}$ MK-2206, $50 \mathrm{nM}$ everolimus (everol), $100 \mathrm{nM}$ rapamycin (rapa). Apoptotic cells are illustrated as AnnexinV positive cells in percent. All data represent at least two independent experiments and were displayed as mean $+/$-SEM; *, p $<0.05 ; * *, \mathrm{p}<0.01 ; * * *, \mathrm{p}<0.001$ compared to the solvent (solv); \#, p<0.05 compared to cells treated with either drug alone. Comparisons were made with ANOVA/Tukey's testing. 


\subsubsection{Effects on PI3K/AKT/mTOR signaling, on AMPK activity and on}

\section{LC3-II protein levels in RD cells}

Protein analysis of RD cells after treatment with low doses of SMO antagonists $(10 \mu \mathrm{M})$ plus $\mathrm{PI} 3 \mathrm{~K} / \mathrm{AKT} / \mathrm{mTOR}$ inhibitors revealed that, as expected, single incubation with PI103, GDC-0941 and MK-2206 inhibited phosphorylation of AKT (Figure 18). Interestingly, the mTOR inhibitors everolimus and rapamycin stimulated phosphorylation of AKT. The activity of mTOR was repressed by everolimus and rapamycin as well as by PI103 and GDC-0941 (revealed by a decrease in pS6). In contrast, MK-2206 did not modulate the pS6 protein level. In addition, pAMPK/AMPK levels were not regulated by the compounds. The exception was PI103 which slightly decreased it. Caspase 3 was activated by PI103 and to a lesser extent by GDC-0941 and MK-2206. Induction of LC3-II was observed by all PI3K/AKT/mTOR inhibitors however to a variable range. The most pronounced increase in LC3-II was seen with PI103, GDC-0941 and MK-2206 (Figure 18).

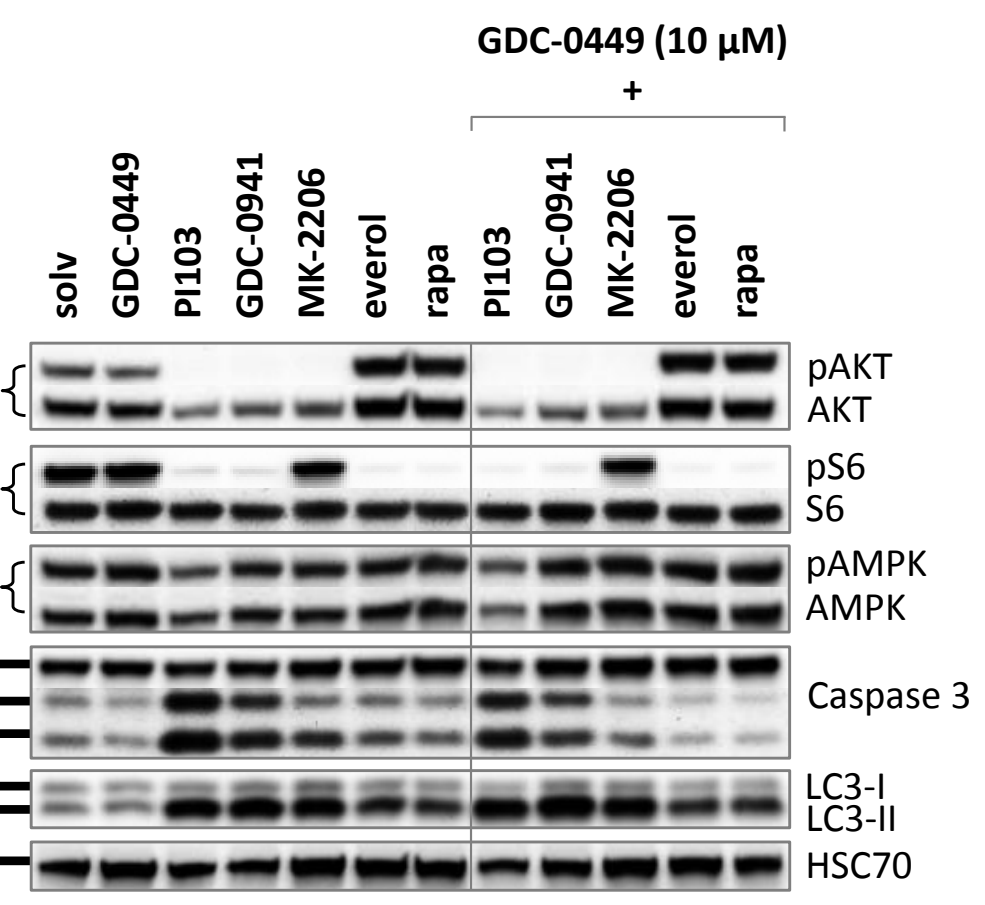

Figure 18: Effects of GDC-0449 and/or PI3K/AKT/mTOR inhibitors on PI3K/AKT/mTOR signaling, phosphorylation status of AMPK and LC3-II protein levels. Western blot analysis of RD cells incubated for $48 \mathrm{~h}$ with $10 \mu \mathrm{M}$ GDC-0449, $3 \mu \mathrm{M}$ PI103, $10 \mu \mathrm{M}$ GDC-0941, $5 \mu \mathrm{M}$ MK-2206, $50 \mathrm{nM}$ everolimus (everol), $100 \mathrm{nM}$ rapamycin (rapa) and with the drug combination as indicated. HSC70 expression levels served as loading control. The data represent the results from one experiment. solv, solvent. 
The combination of PI3K/AKT/mTOR inhibitors with GDC-0449 in RD cells (Figure 18) did not alter the analyzed protein levels compared to the drugs alone and hence provided no improvement over the single drug treatment. It rather seemed that the active caspase 3 levels induced by PI103, GDC-0941 and MK-2206 were reduced in combination with GDC-0449.

Incubation with $10 \mu \mathrm{M}$ LDE225 in combination with PI3K/AKT/mTOR inhibitors did not markedly alter the analyzed parameters, i.e. no cooperative effects were observed (Figure 19). However, similarly to GDC-0449, caspase 3 activity induced by PI103, GDC-0941 and MK-2206 seemed to be slightly decreased in combination with LDE225.

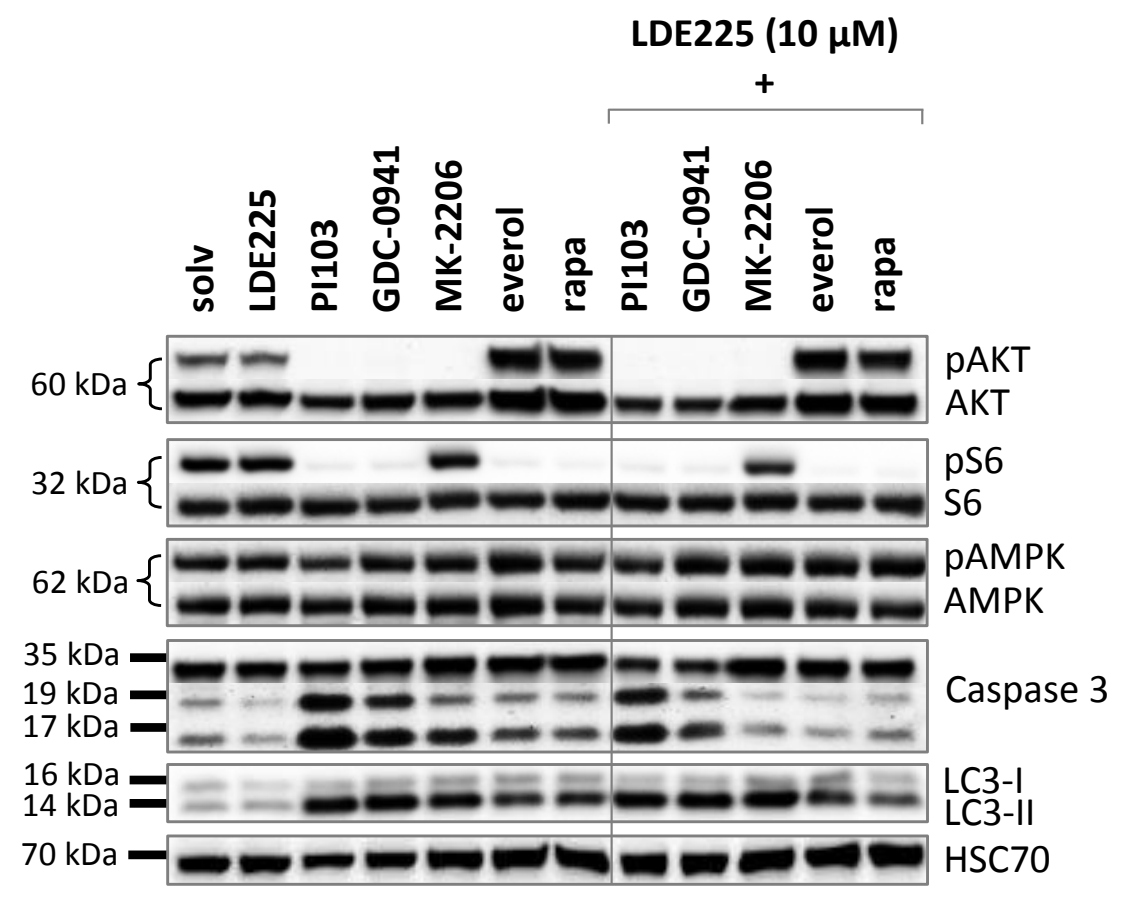

Figure 19: Effects of LDE225 and/or PI3K/AKT/mTOR inhibitors on PI3K/AKT/mTOR signaling phosphorylation status of AMPK and LC3-II protein levels. Western blot analysis of RD cells incubated for $48 \mathrm{~h}$ with $10 \mu \mathrm{M}$ LDE225, $3 \mu \mathrm{M}$ PI103, $10 \mu \mathrm{M}$ GDC-0941, $5 \mu \mathrm{M}$ MK-2206, $50 \mathrm{nM}$ everolimus (everol), $100 \mathrm{nM}$ rapamycin (rapa) and with the combination as indicated. HSC70 expression levels served as loading control. The data represent the results from one experiment. solv, solvent.

In contrast to GDC-0449 and LDE225, the combination of HhA plus everolimus and rapamycin resulted in reduction of pAKT comparable to solvent levels and a slight decrease of AMPK phosphorylation was observed when combining HhA plus PI103, GDC-0941 or MK-2206 (Figure 20). In addition, HhA also repressed caspase 3 activity induced by PI103 and GDC-0941. 


\section{RESULTS}

However it strongly enhanced MK-2206 induced caspase 3 activity. These findings correspond to the data of the apoptosis assay, in which combination of $10 \mu \mathrm{M}$ HhA and MK-2206 strongly increased the number of AnnexinV positive cells compared to the single drug treatments (Figure $17 \mathrm{C})$. HhA also cooperated with all drugs in the induction of LC3-II protein levels, which was in contrast to GDC-0449 and LDE225. It should be noted that the latter western blot analyses are preliminary. Thus, the respective experiments have to be repeated in the future.

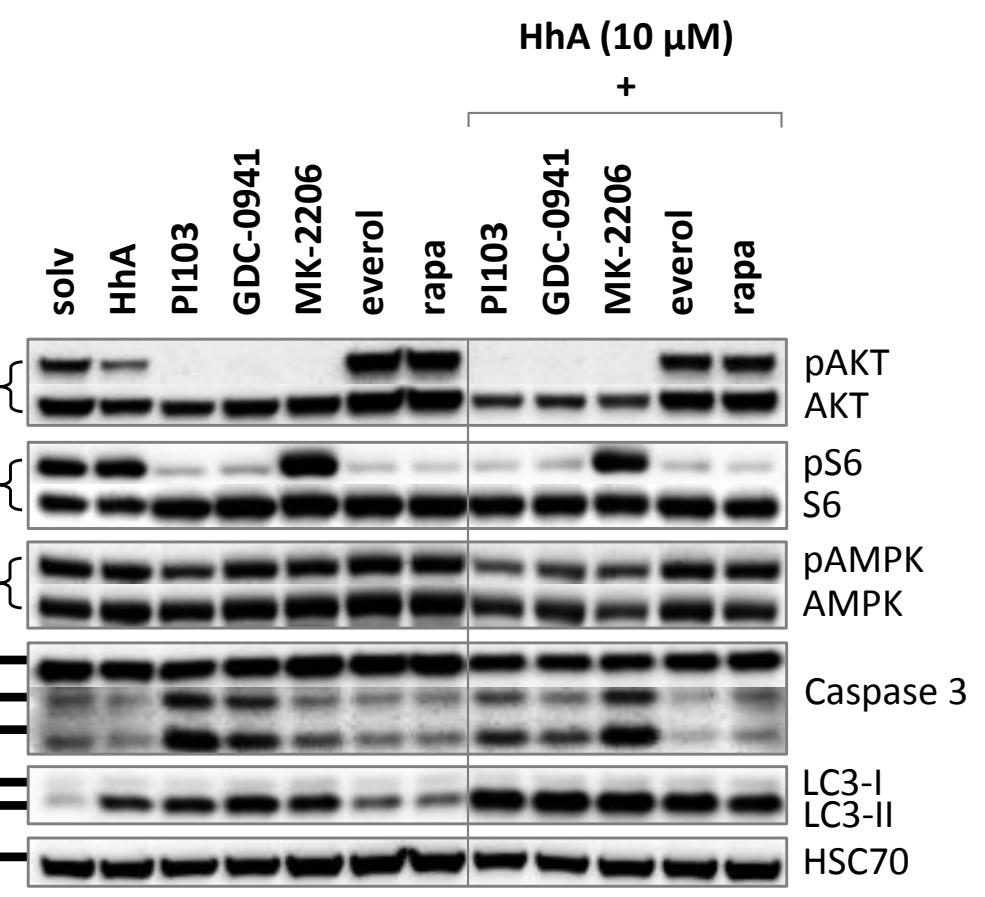

Figure 20: Effects of HhA and/or PI3K/AKT/mTOR inhibitors on PI3K/AKT/mTOR signaling, phosphorylation status of AMPK and LC3-II protein levels. Western blot analysis of RD cells incubated for $48 \mathrm{~h}$ with $10 \mu \mathrm{M}$ HhA, $3 \mu \mathrm{M}$ PI103, $10 \mu \mathrm{M}$ GDC-0941, $5 \mu \mathrm{M}$ MK-2206, $50 \mathrm{nM}$ everolimus (everol), $100 \mathrm{nM}$ rapamycin (rapa) and with the drug combination as indicated. HSC70 expression levels served as loading control. The data represent the results from one experiment. solv, solvent.

In conclusion, the data suggest that $\mathrm{HhA}$ is the most efficient compound for combination therapy with PI3K/AKT/mTOR inhibitors. This assumption is based on its cooperative proapoptotic effects with several of the used inhibitors (especially seen for the combination HhA plus MK-2206, Figure 17 C, Figure 20). HhA also exerted combination effects that could be of relevance in antitumor therapies e.g. it reversed the phosphorylation of AKT mediated by mTOR inhibitors and reduced AMPK phosphorylation when combined with PI103, GDC-0941 or MK-2206 (Figure 20). In addition as a single drug HhA showed antiproliferative and proapoptotic effects, inhibited pAKT and induced LC3-II. Hence in the following experiments, 
the effects of HhA plus $\mathrm{PI} 3 \mathrm{~K} / \mathrm{AKT} / \mathrm{mTOR}$ inhibitors were studied in more detail and in additional human RMS cell lines.

\subsubsection{Effects of HhA plus PI3K/AKT/mTOR inhibitors in RD, RUCH-2, RMS-13 and Rh41 cells}

The following investigations focused on possible cooperative effects of the SMO antagonist HhA in combination with PI3K/AKT/mTOR inhibitors in different RMS cells. Since $30 \mu \mathrm{M}$ HhA showed more potency to reduce cellular proliferation and to induce apoptosis in all RMS cell lines than compared to $10 \mu \mathrm{M}$ HhA, the higher dose was used for the next investigations.

\subsubsection{Effects on HH signaling activity, IGF2 expression and muscle differentiation markers}

In RD cells $30 \mu \mathrm{M}$ HhA significantly inhibited $\mathrm{HH}$ signaling activity. $\mathrm{HH}$ signaling inhibition was also seen for all used PI3K/AKT/mTOR inhibitors (Figure $21 \mathrm{~A}$ ). In combination with PI103 or rapamycin HhA showed a cooperative effect on GLII expression compared to the corresponding single drug treatments. Paradoxically, GLII expression was significantly increased when HhA was combined with MK-2206.

In contrast to RD cells, single treatment with $\mathrm{HhA}$ did not significantly reduced $\mathrm{HH}$ signaling activity in RUCH-2 cells. However, similar to RD cells all PI3K/AKT/mTOR inhibitors deceased GLI1 expression in RUCH-2 cells (Figure $21 \mathrm{~B}$ ). The strongest reduction was revealed with GDC-0941, which was similar to RD cells. However, cooperative effects on GLII repression were not observed when $\mathrm{HhA}$ was combined with PI3K/AKT/mTOR inhibitors (Figure $21 \mathrm{~B}$ ).

In RMS-13 cells GLII transcription was reduced by PI103, GDC-0941, MK-2206 and rapamycin, whereas HhA and everolimus had no effect (Figure $21 \mathrm{C}$ ). Cooperative effects on GLII expression were not revealed.

Analysis of Rh41 cells showed that PI103 and GDC-0941 inhibited HH signaling activity, whereas MK-2206 and the mTOR inhibitors did not (Figure 21 D). In this cell line HhA paradoxically increased GLII expression. Similar to RUCH-2 and RMS-13 cells, cooperative effects were not observed. 

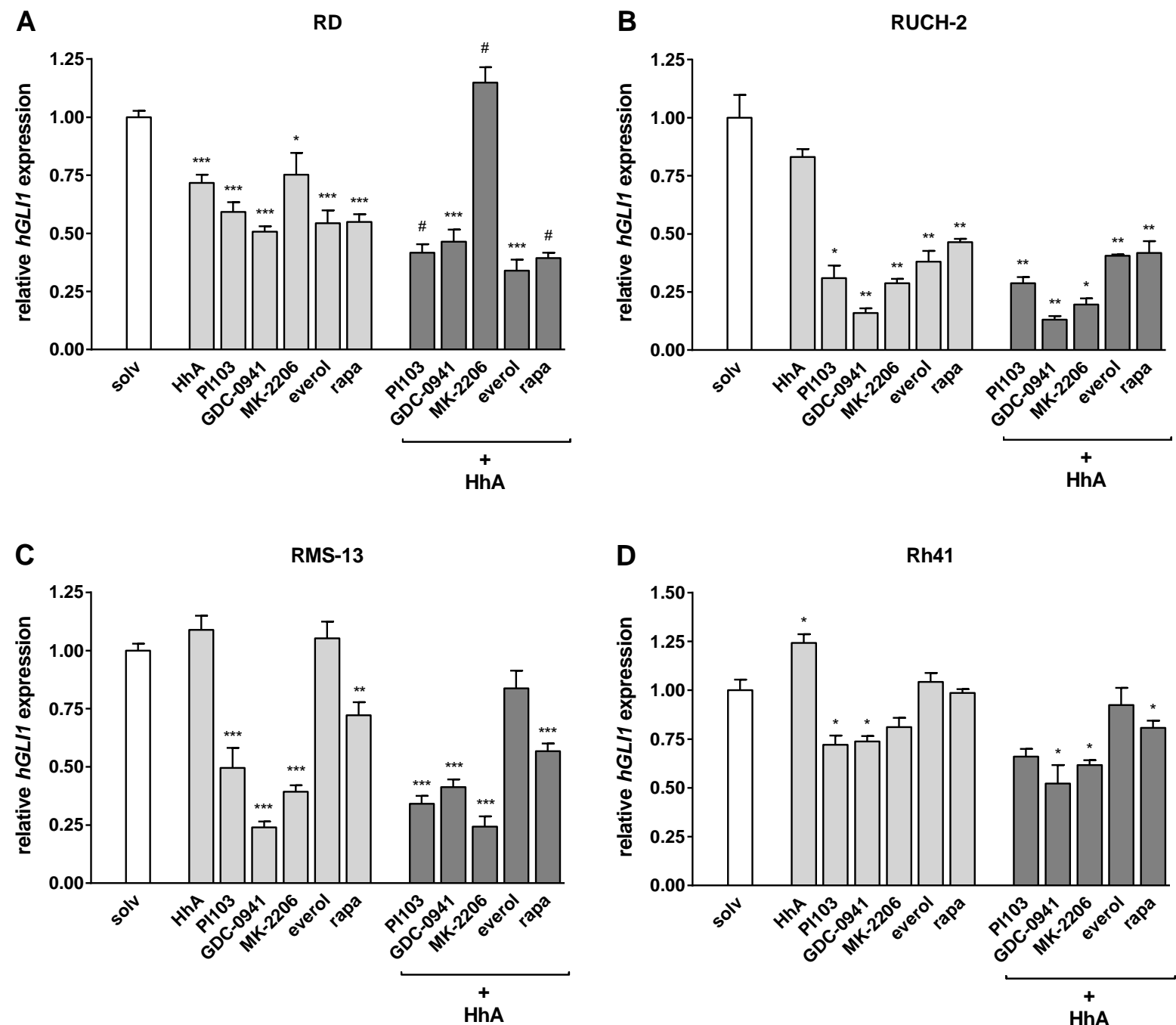

Figure 21: Modulation of HH signaling activity by HhA and/or PI3K/AKT/mTOR inhibitors. Relative GLII expression in RD (A), RUCH-2 (B), RMS-13 (C) and Rh41 (D) cells after incubation (24 h) with $30 \mu \mathrm{M} \mathrm{HhA,} 3 \mu \mathrm{M}$ PI103 (200 nM for Rh41), $10 \mu \mathrm{M}$ GDC-0941 (500 nM for Rh41), $5 \mu \mathrm{M}$ MK-2206 (1 $\mu \mathrm{M}$ for RMS-13, Rh41), $50 \mathrm{nM}$ everolimus (everol), $100 \mathrm{nM}$ rapamycin (rapa) or in combination as indicated. GLII expression was normalized to $18 \mathrm{~S}$ rRNA expression levels. The corresponding solvent controls (solv) were set to 1 . The data represent three independent experiments for RD, two for RMS-13 and one experiment for RUCH-2 and Rh41 cells. The data are displayed as mean +/-SEM; *, $\mathrm{p}<0.05 ; * *, \mathrm{p}<0.01 ; * * *, \mathrm{p}<0.001$ compared to the solvent; \#, $\mathrm{p}<0.05$ compared to cells treated with either drug alone. Comparisons were made with ANOVA/Tukey's testing.

In RD cells IGF2 expression was inhibited upon single drug treatment using HhA, PI103 and GDC-0941, whereas MK-2206 had no effect (Figure 22 A). In contrast, the mTOR inhibitors everolimus and rapamycin induced the expression of IGF2. Combinations with HhA did not significantly enhance these effects compared to single drug incubations, except for the combination of HhA plus rapamycin that restored IGF2 expression to basal levels. Since 
RUCH-2 did not express IGF2, its transcription was not analyzed. In RMS-13 cells IGF2 expression was inhibited by GDC-0941 and MK-2206 and induced by everolimus (Figure 22 B). Cooperative effects were not achieved. In Rh41 cells only PI103 inhibited IGF2 transcription (Figure $22 \mathrm{C}$ ). Similar to RD cells IGF2 expression was stimulated by rapamycin. Even though there were no significant cooperative effects, the combination of HhA with PI3K/AKT/mTOR inhibitors seemed to slightly suppress IGF2 expression compared to the single drug treatments.
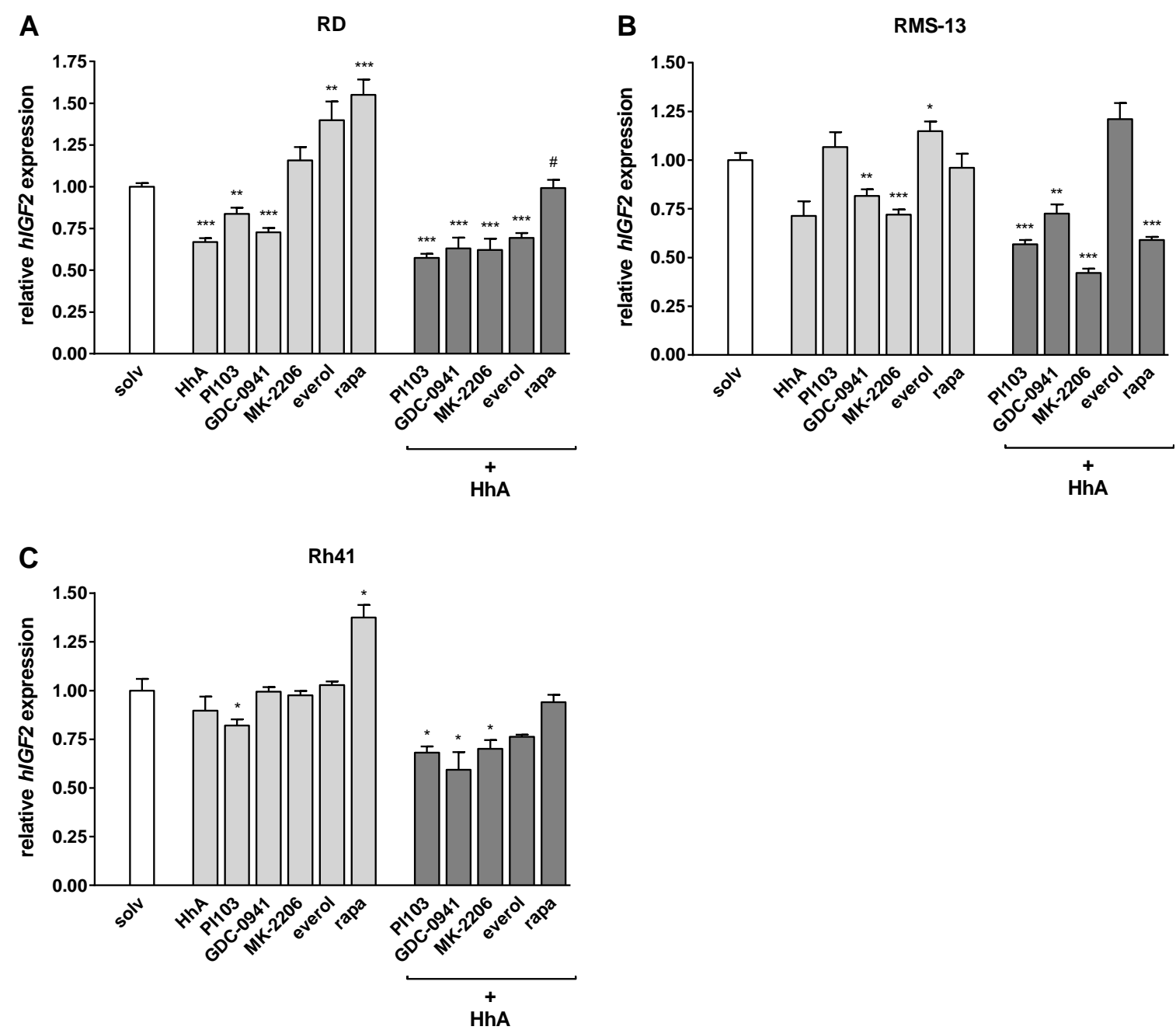

Figure 22: Modulation of IGF2 transcription by HhA and/or PI3K/AKT/mTOR inhibitors. Relative IGF2 expression in RD (A), RMS-13 (B) and Rh41 (C) cells after incubation (24 h) with $30 \mu \mathrm{M}$ HhA, $3 \mu \mathrm{M}$ PI103 (200 nM for Rh41), $10 \mu$ M GDC-0941 (500 nM for Rh41), $5 \mu$ M MK-2206 (1 $\mu$ M for RMS-13, Rh41), $50 \mathrm{nM}$ everolimus (everol), $100 \mathrm{nM}$ rapamycin (rapa) or in combination as indicated. IGF2 expression was normalized to $18 S$ rRNA expression levels. The corresponding solvent controls (solv) were set to 1 . The data represent three independent experiments for RD, two for RMS-13 and one experiment for Rh41 cells. The data are displayed as mean +/-SEM; *, $\mathrm{p}<0.05 ; * *, \mathrm{p}<0.01 ; * * *, \mathrm{p}<0.001$ compared to the solvent; \#, $\mathrm{p}<0.05$ compared to cells treated with either drug alone. Comparisons were made with ANOVA/Tukey's testing. 


\section{RESULTS}

Because RUCH-2 cells neither expressed $M Y O D$ nor $M Y H 1$, expression analyses of these genes was not possible.

The expression of MYOD in RD cells was not altered by MK-2206 and mTOR inhibitors (Figure 23 A). However, MYOD expression was significantly decreased by single incubation with PI103 and GDC-0941. The combination with HhA that itself decreased MYOD expression, did not further promote these effects compared to single drug treatment. The exception was the combination HhA plus GDC-0941, which significantly enhanced MYOD transcription above the respective single drug levels (Figure 23 A). In RMS-13 cells no significant effects on MYOD transcription were observed for any of the drugs or drug combinations (Figure $23 \mathrm{~B}$ ). In Rh41 cells MYOD expression was decreased by everolimus and increased by GDC-0941 and MK-2206 (Figure $23 \mathrm{C}$ ). Again no cooperative effects were determined.

In RD cells the expression of MYH1 was inhibited by all drugs (Figure 24 A). Thus, PI103, GDC-0941 and MK-2206 reduced MYH1 expression by approximately $85 \%$ compared to the solvent. These effects were not further enhanced upon addition of HhA. In RMS-13 MYH1 transcription was inhibited by HhA, PI103 and GDC-0941 (Figure 24 B). In contrast, MK-2206 and everolimus slightly induced $M Y H 1$ expression while rapamycin had no effect. Interestingly, the combination of HhA plus MK-2206 almost completely blocked MYHI expression and significantly cooperated in this suppression. No other cooperative effects were observed (Figure $24 \mathrm{~B})$. In Rh41 cells a significant inhibition of $M Y H 1$ expression was seen after single treatment with HhA or by the combination HhA plus MK-2206 (Figure 24 C), however without any cooperative effect. 
A

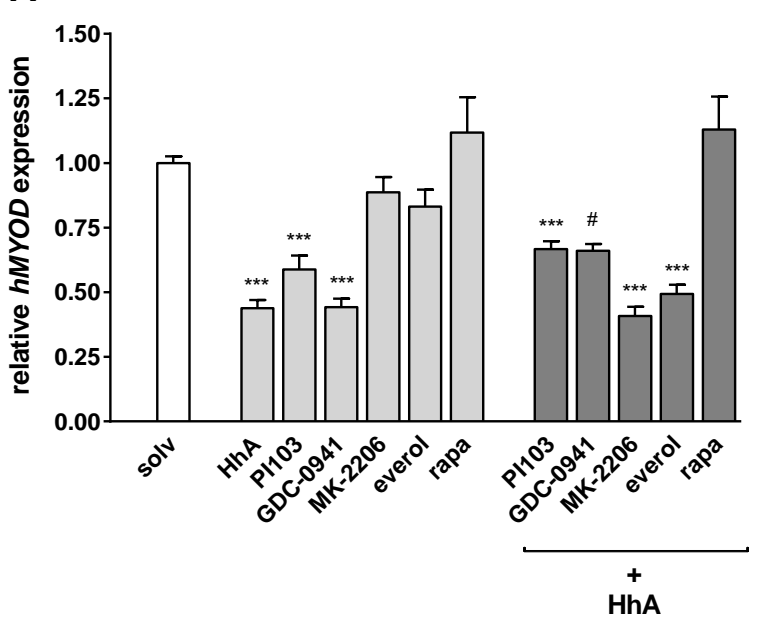

C

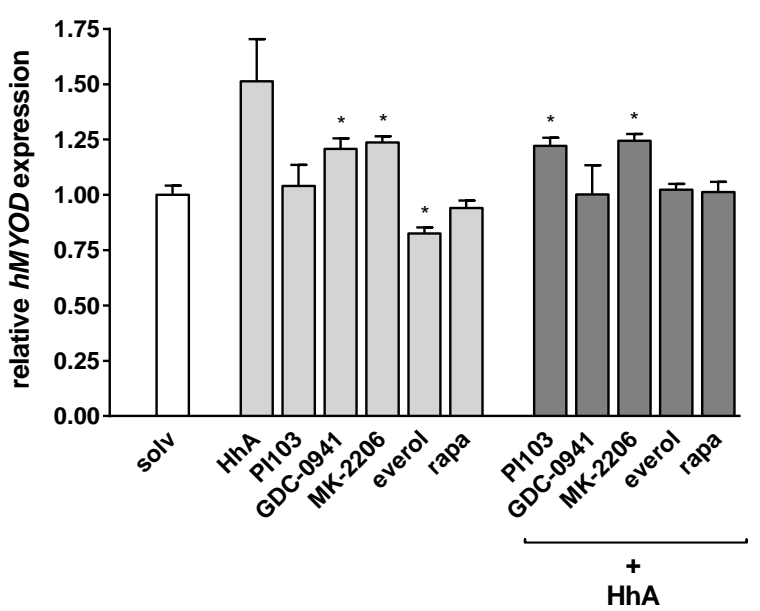

B RMS-13

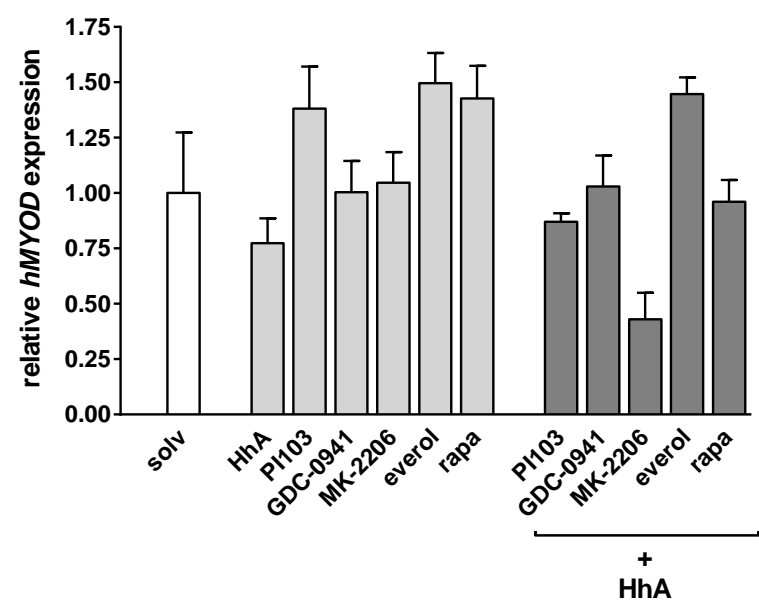

Figure 23: Modulation of MYOD transcription by HhA and/or PI3K/AKT/mTOR inhibitors. Relative MYOD expression in RD (A), RMS-13 (B) and Rh41 (C) cells after incubation (24 h) with $30 \mu \mathrm{M}$ HhA, $3 \mu \mathrm{M}$ PI103 (200 nM for Rh41), $10 \mu \mathrm{M}$ GDC-0941 (500 nM for Rh41), $5 \mu$ M MK-2206 (1 $\mu$ M for RMS-13, Rh41), $50 \mathrm{nM}$ everolimus (everol), $100 \mathrm{nM}$ rapamycin (rapa) or in combination as indicated. MYOD expression was normalized to $18 S$ rRNA expression levels. The corresponding solvent controls (solv) were set to 1 . The data represent three independent experiments for RD, two for RMS-13 and one experiment for Rh41 cells. The data are displayed as mean +/-SEM; *, p <0.05; ***, $<<0.001$ compared to the solvent; \#, $\mathrm{p}<0.05$ compared to cells treated with either drug alone. Comparisons were made with ANOVA/Tukey's testing. 
A

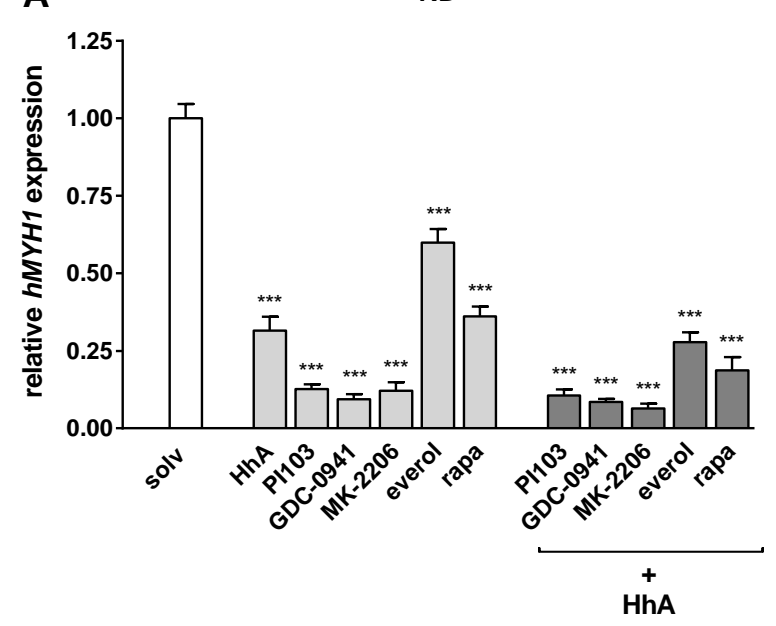

C

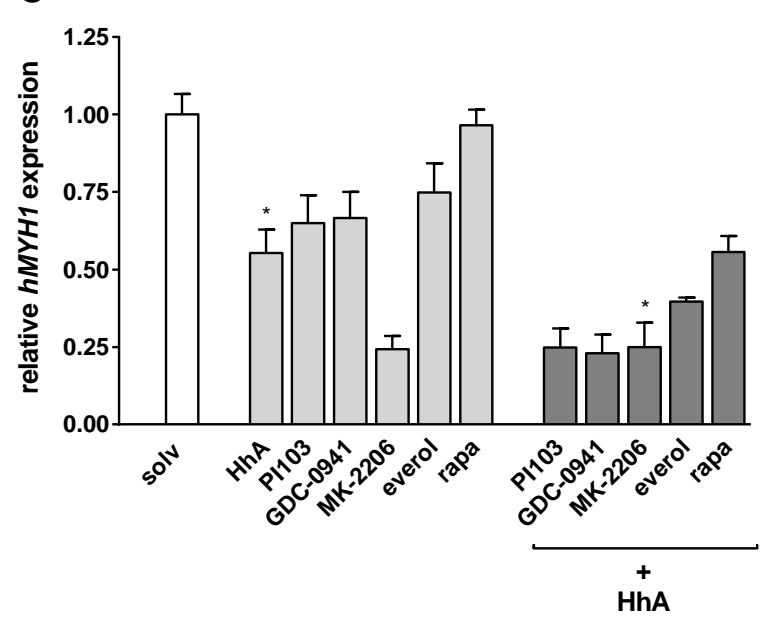

B

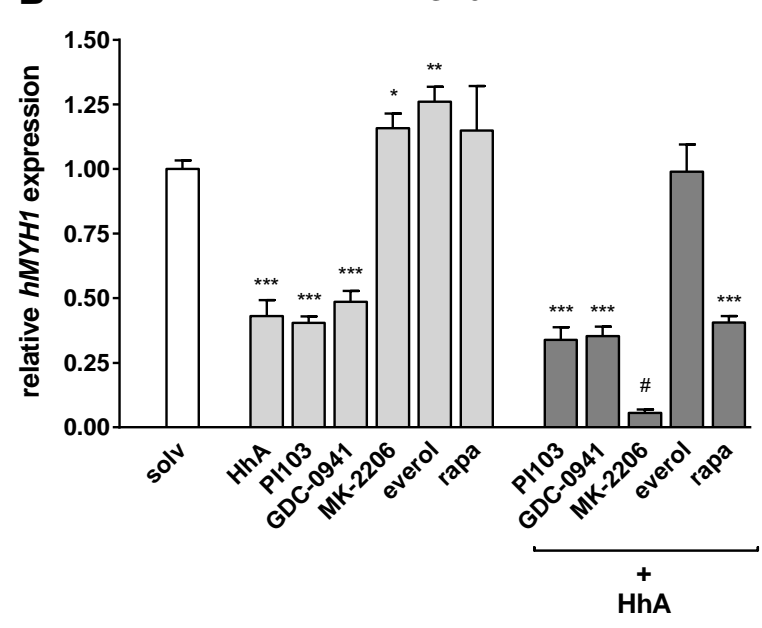

Figure 24: Modulation of MYH1 transcription by HhA and/or PI3K/AKT/mTOR inhibitors. Relative MYHI expression in RD (A), RMS-13 (B) and Rh41 (C) cells after incubation (24 h) with $30 \mu \mathrm{M}$ HhA, $3 \mu \mathrm{M}$ PI103 (200 nM for Rh41), $10 \mu \mathrm{M}$ GDC-0941 (500 nM for Rh41), $5 \mu$ M MK-2206 (1 $\mu$ M for RMS-13, Rh41), $50 \mathrm{nM}$ everolimus (everol), $100 \mathrm{nM}$ rapamycin (rapa) or in combination as indicated. $M Y H 1$ expression was normalized to $18 S$ rRNA expression levels. The corresponding solvent controls (solv) were set to 1 . The data represent three independent experiments for RD, two for RMS-13 and one experiment for Rh41 cells. The data are displayed as mean +/-SEM; *, $\mathrm{p}<0.05 ; * *, \mathrm{p}<0.01 ;{ }^{* * *}, \mathrm{p}<0.001$ compared to the solvent; \#, $\mathrm{p}<0.05$ compared to cells treated with either drug alone. Comparisons were made with ANOVA/Tukey's testing.

Together, the data show that the PI3K/AKT/mTOR inhibitors can decrease HH signaling activity, and that the strongest inhibition of GLII expression is mediated by GDC-0941 in almost all RMS cell lines (Figure 21). Indeed, GDC-0941 seems to have more potency to block GLII transcription than the $\mathrm{HH}$ inhibitor HhA (according to the used concentrations). GDC-0941 also inhibits IGF2 transcription in two of the three RMS cell lines analyzed (Figure 22). Furthermore 
the data demonstrate that mTOR inhibitors can induce $I G F 2$ transcription in all three analyzed cell lines (Figure 22). Furthermore, all used inhibitors have the potency to modulate muscle differentiation, which however again is depended on the used cell line. Since the respective gene expressions in RUCH-2 and Rh41 cells were only measured once, the data should be judged with caution and the experiments have to be repeated in the future (see respective figure legends).

\subsubsection{Effects on proliferation and apoptosis}

In RD cells single drug treatment with HhA, PI103 and GDC-0941 significantly reduced the proliferation rate by $35 \%, 20 \%$ and $25 \%$, respectively (Figure $25 \mathrm{~A}$ ). In contrast and surprisingly, MK-2206 and both mTOR inhibitors significantly increased the proliferation rate of RD cells. However, when the latter drugs were combined with HhA, proliferation levels went down to HhA induced levels and a cooperative antiproliferative effect was detected for the combination of HhA plus MK-2206. Such a cooperative antiproliferative effect was also seen for the combination of HhA plus GDC-0941.

Similar to RD cells single drug treatment using HhA, PI103 or GDC-0941 significantly inhibited cellular proliferation of RUCH-2 cells, whereas MK-2206, everolimus and rapamycin did not (Figure 25 B). Again the combination HhA plus GDC-0941 or MK-2206 strongly inhibited proliferation by $75 \%$ and $80 \%$, respectively, which was significant compared to the single drug treatments.

In RMS-13 cells significant antiproliferative effects were revealed with HhA, PI103 and GDC-0941 (Figure 25 C). In contrast everolimus did not impact on proliferation, whereas MK-2206 and rapamycin increased the proliferation rate. When the latter drugs were combined with $\mathrm{HhA}$, proliferation rates went down to HhA induced levels. A cooperative antiproliferative effect was detected when HhA was combined with PI103. Otherwise no cooperative effects were obvious.

In Rh41 cells HhA, PI103, GDC-0941 and also MK-2206 inhibited proliferation, while mTOR inhibitors did not (Figure $25 \mathrm{D}$ ). In this cell line cooperative antiproliferative effects were observed for HhA in combination with all other compounds. 
A

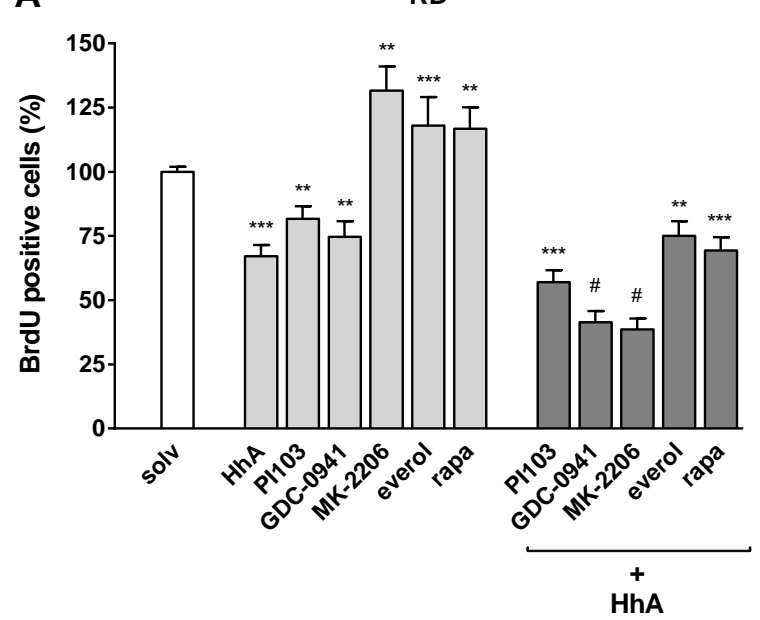

C

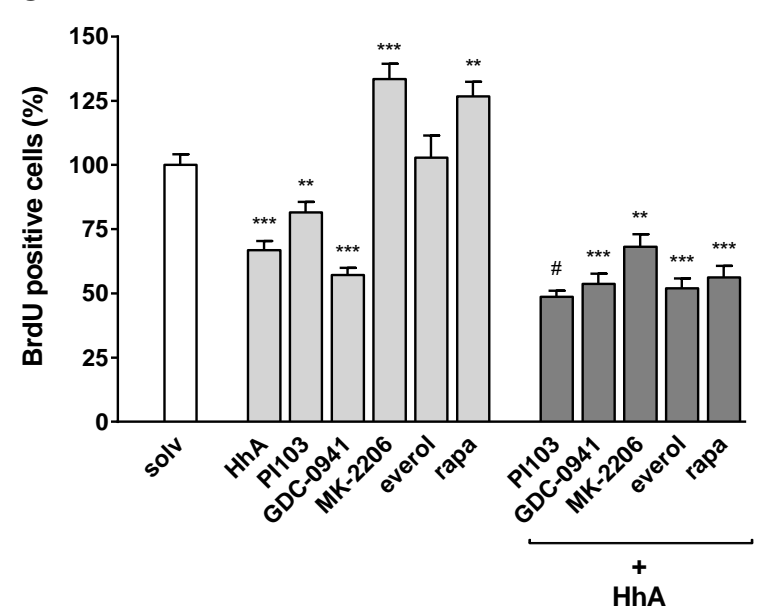

B

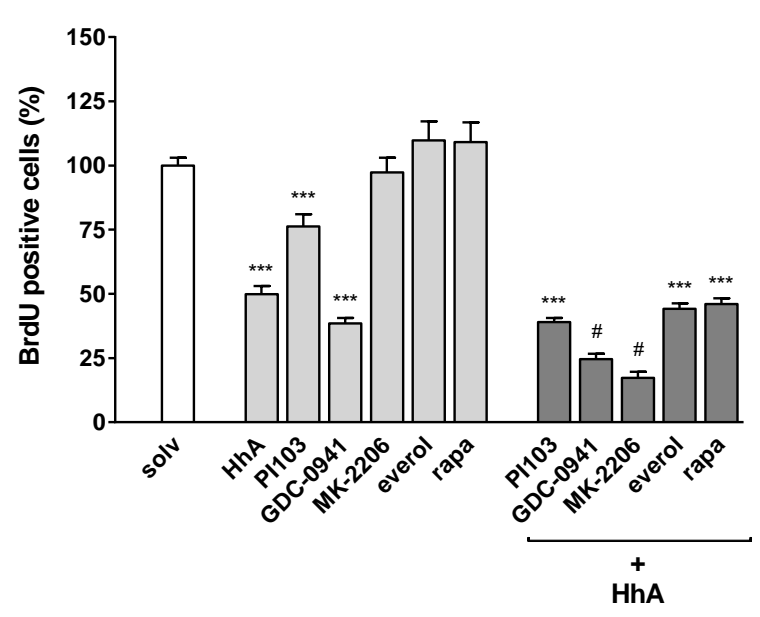

D

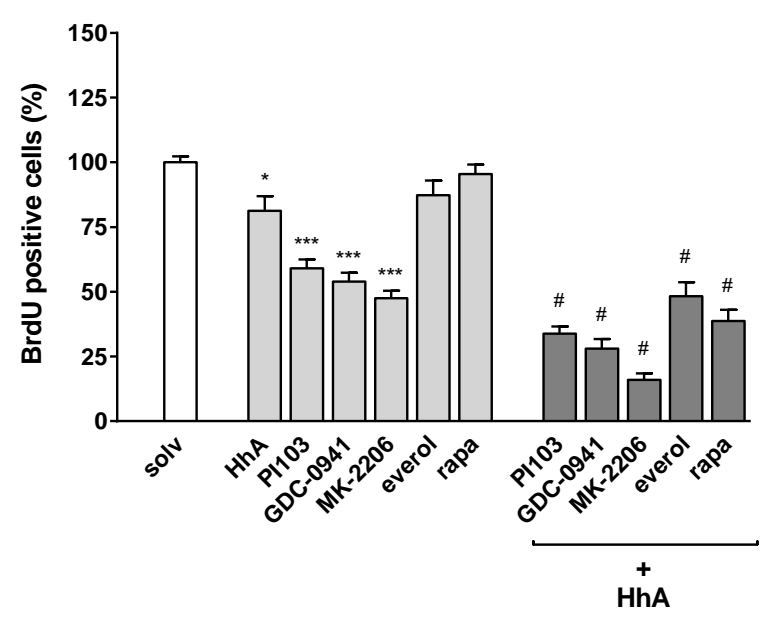

Figure 25: Modulation of proliferation by $\mathrm{HhA}$ and/or PI3K/AKT/mTOR inhibitors. Measurement of BrdU incorporation in RD (A), RUCH-2 (B), RMS-13 (C) and Rh41 (D) cells after incubation (24 h) with $30 \mu \mathrm{M} \mathrm{HhA,}$ $3 \mu$ M PI103 (200 nM for Rh41), $10 \mu$ M GDC-0941 (500 nM for Rh41), $5 \mu$ M MK-2206 (1 $\mu$ M for RMS-13, Rh41), $50 \mathrm{nM}$ everolimus (everol), $100 \mathrm{nM}$ rapamycin (rapa) or in combination as indicated. BrdU incorporation is presented as percentage of respective solvent controls (solv) that were set to $100 \%$. The data shown summarize at least three independent experiments and are represented as mean +/-SEM; *, p $<0.05$; **, p $<0.01 ; * * *, p<0.001$ compared to the solvent; \#, $\mathrm{p}<0.05$ compared to cells treated with either drug alone. Comparisons were made with ANOVA/Tukey's testing.

Subsequently the impact of HhA plus PI3K/AKT/mTOR inhibitors was compared concerning their potential to induce apoptosis. As already shown in Figure $17 \mathrm{C}$, in RD cells single drug treatment using HhA, PI103 and GDC-0941 significantly increased the number of AnnexinV positive cells (Figure $26 \mathrm{~A}$ ). A significant cooperative proapoptotic effect was observed for HhA plus MK-2206. In RMS-13 cells HhA, PI103 and GDC-0941 significantly induced apoptosis, 
whereas MK-2206, everolimus and rapamycin had no proapoptotic effect (Figure 26 B). A significant increase in AnnexinV positive cells compared to the single drug treatments was revealed by the combinations HhA plus PI103, HhA plus GDC-0941 or plus MK-2206. In Rh41 cells a stimulation of apoptosis was caused by HhA, PI103, GDC-0941 and MK-2206 (Figure 26 C). Similar to the other RMS cell lines the mTOR inhibitors did not activate apoptosis. However, a significant cooperative proapoptotic effect of HhA with the other drugs was not observed in Rh41 cells.

A

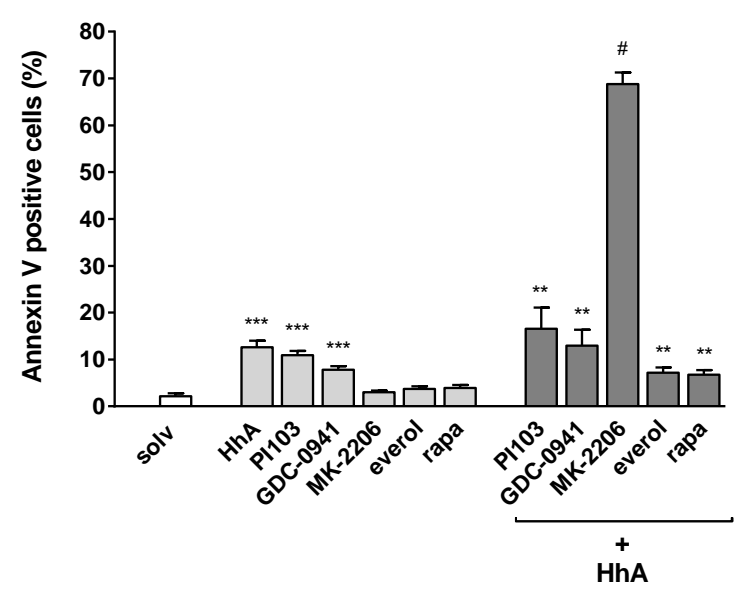

C

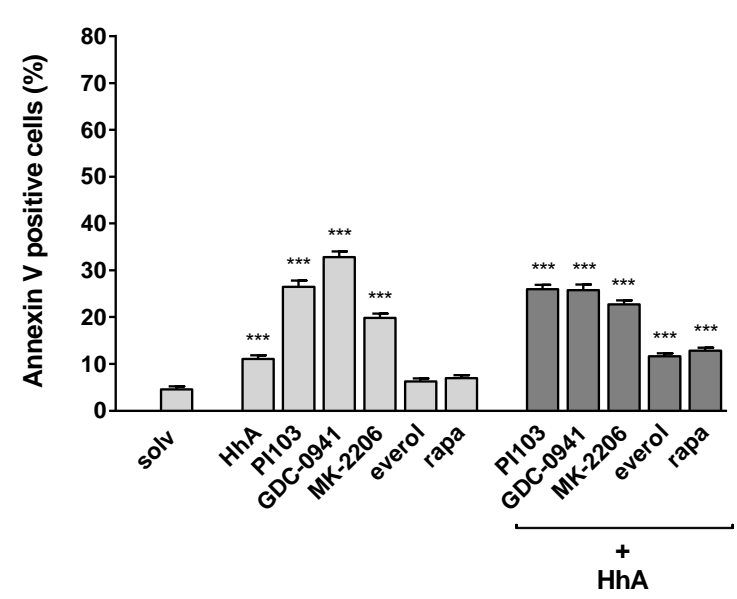

B $\quad$ RMS-13

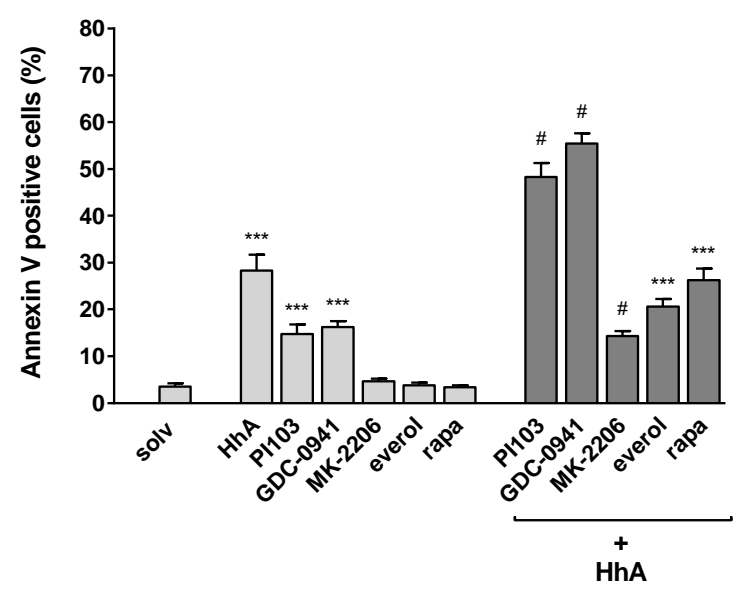

Figure 26: Modulation of apoptosis by HhA and/or PI3K/AKT/mTOR inhibitors. RD (A), RMS-13 (B) and Rh41 (C) cells were incubated for $48 \mathrm{~h}$ with $30 \mu \mathrm{M}$ HhA, $3 \mu \mathrm{M}$ PI103 (200 nM for Rh41), $10 \mu \mathrm{M}$ GDC-0941 (500 nM for Rh41), $5 \mu \mathrm{M}$ MK-2206 ( $1 \mu \mathrm{M}$ for RMS-13, Rh41), $50 \mathrm{nM}$ everolimus (everol), $100 \mathrm{nM}$ rapamycin (rapa) or in combination as indicated. Apoptotic cells are illustrated as AnnexinV positive cells in percent. All data represent at least three independent experiments measured in duplicates and were displayed as mean +/-SEM; *, $\mathrm{p}<0.05 ;{ }^{* *}, \mathrm{p}<0.01 ; * * *, \mathrm{p}<0.001$ compared to the solvent (solv); \#, $\mathrm{p}<0.05$ compared to cells treated with either drug alone. Comparisons were made with ANOVA/Tukey's testing. 


\section{RESULTS}

Together these data demonstrate that a single drug treatment using HhA, PI103 or GDC-0941 inhibits proliferation of the cell lines used in this study (Figure 25) and induces proapoptotic effects (Figure 26). In contrast, mTOR inhibitors increase the proliferation rate of almost all cell lines and do not modulate apoptosis. Moreover, substantial cooperative antiproliferative effects are induced by the combination of HhA plus PI103, HhA plus GDC-0941 or HhA plus MK-2206 in almost all cell lines. In addition, the combination HhA plus MK-2206 strongly induces apoptosis in RD cells (Figure 26 A) and cooperative proapoptotic effects are observed for HhA plus PI103 or GDC-0941 in RMS-13 cells (Figure 26 B).

\subsubsection{Effects on PI3K/AKT/mTOR signaling, on AMPK activity and on LC3-II expression}

As observed before in RD cells (see 6.2.2) PI103, GDC-0941 and MK-2206 also inhibited phosphorylation of AKT in all other RMS cell lines (Figure 28, Figure 29, Figure 30). Phosphorylation of S6 was repressed by the mTOR inhibitors everolimus and rapamycin and by PI103 and GDC-0941.

In RD cells single drug treatment with $30 \mu \mathrm{M}$ HhA increased LC3-II expression (Figure 27). This has also been shown for $10 \mu \mathrm{M}$ HhA (Figure 20 in section 6.2.2). However, the effects of $30 \mu \mathrm{M}$ HhA on inhibition of pAKT were more pronounced and $30 \mu \mathrm{M}$ HhA additionally reduced pAMPK and pS6 levels in contrast to $10 \mu \mathrm{M}$ HhA (Figure 27). Although single treatments with everolimus or rapamycin induced pAKT levels, the combination with $30 \mu \mathrm{M}$ HhA reduced it and this effect was stronger than that observed upon combination with $10 \mu \mathrm{M}$ HhA. Moreover, $30 \mu \mathrm{M}$ HhA plus MK-2206 inhibited S6 phosphorylation in contrast to $10 \mu \mathrm{M} \mathrm{HhA}$. Additionally, $30 \mu \mathrm{M}$ HhA in combination with GDC-0941 or MK-2206 also reduced pAMPK levels more potently than $10 \mu \mathrm{M}$ HhA. Treatment with $30 \mu \mathrm{M}$ HhA plus MK-2206 resulted in an enormous increase of caspase 3 activity, which confirmed the results from the AnnexinV experiments (Figure $17 \mathrm{C}$ ). This effect was also stronger than upon combination of MK-2206 with $10 \mu \mathrm{M}$ HhA (Figure 20 in section 6.2.2). Similar to $10 \mu \mathrm{M} \mathrm{HhA}$, the combinations of $30 \mu \mathrm{M}$ HhA with any drug resulted in an increase in LC3-II levels compared to incubations with either drug alone. 


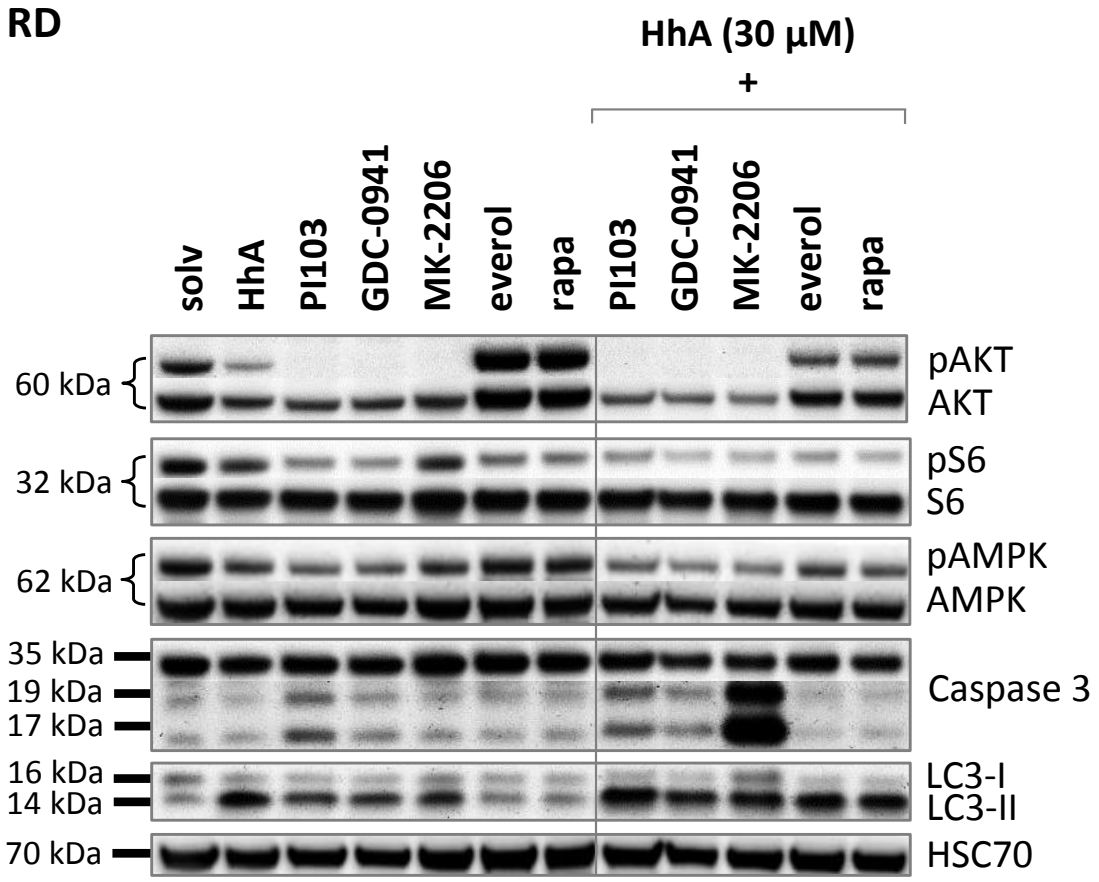

Figure 27: Effects of HhA and/or PI3K/AKT/mTOR inhibitors on PI3K/AKT/mTOR signaling, phosphorylation status of AMPK and LC3-II protein levels in RD cells. Western blot analysis of RD cells incubated for $48 \mathrm{~h}$ with $30 \mu \mathrm{M}$ HhA, $3 \mu \mathrm{M}$ PI103, $10 \mu \mathrm{M}$ GDC-0941, $5 \mu \mathrm{M}$ MK-2206, $50 \mathrm{nM}$ everolimus (everol), $100 \mathrm{nM}$ rapamycin (rapa) and with the drug combination as indicated. HSC70 expression levels served as loading control. Representative blots of two independent experiments are shown. solv, solvent.

In RUCH-2 cells (Figure 28) the effects of the drugs were similar as in RD cells. The exceptions were everolimus and rapamycin which did not activate AKT. Moreover phosphorylation of S6 was not inhibited by HhA in RUCH-2 cells and activation of caspase 3 by PI103 was not observed. Furthermore HhA plus MK-2206 did not block phosphorylation of S6. Activation of caspase 3 was induced by the combination of HhA plus PI103 or plus GDC-0941. This was in contrast to RD cells, in which activation of caspase 3 was revealed only by the combination HhA plus MK-2206 (Figure 27). Cooperative effects of HhA plus GDC-0941 or HhA plus MK-2206 with respect to inhibition of AMPK phosphorylation was not observed in RUCH-2 cells. The combinations of HhA with PI103, GDC-0941, everolimus or rapamycin resulted in increased LC3-II levels compared to incubations with either drug alone. 


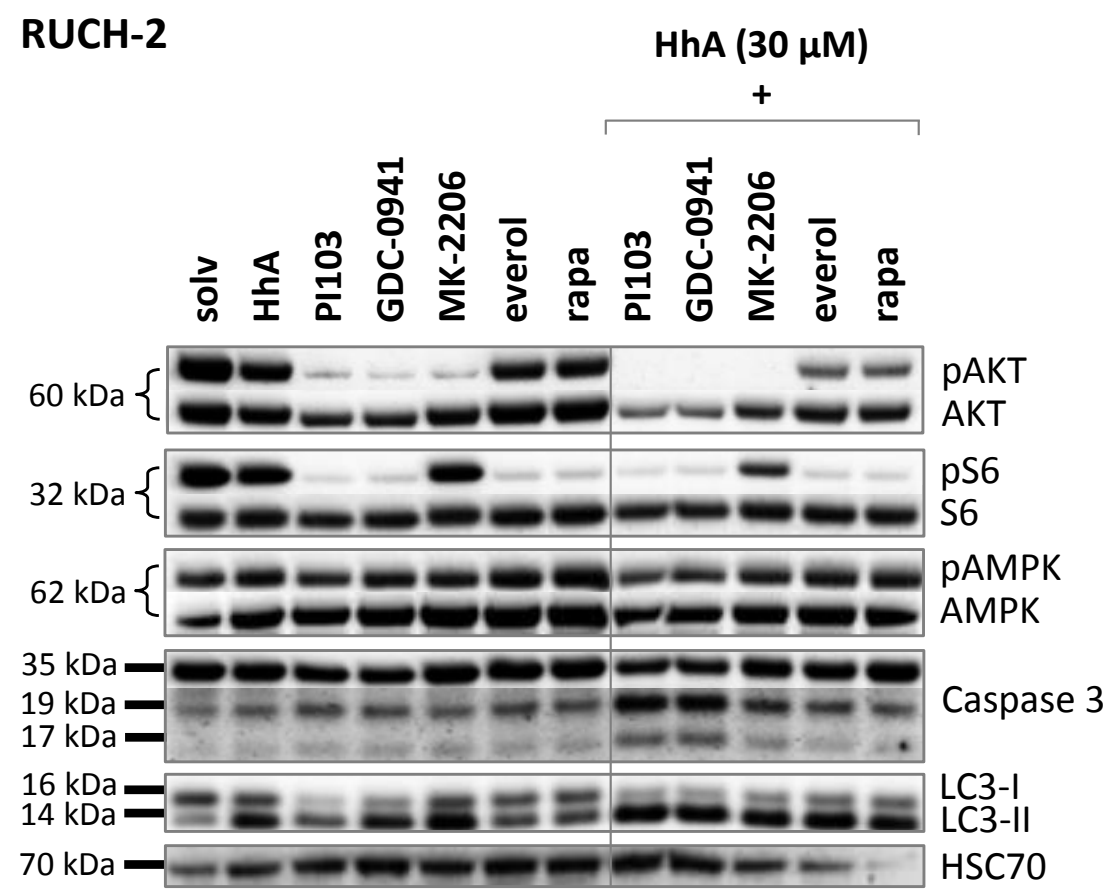

Figure 28: Effects of HhA and/or PI3K/AKT/mTOR inhibitors on PI3K/AKT/mTOR signaling, phosphorylation status of AMPK and LC3-II protein levels in RUCH-2 cells. Western blot analysis of RUCH-2 cells incubated for $48 \mathrm{~h}$ with $30 \mu \mathrm{M}$ HhA, $3 \mu \mathrm{M}$ PI103, $10 \mu \mathrm{M}$ GDC-0941, $5 \mu \mathrm{M}$ MK-2206, $50 \mathrm{nM}$ everolimus (everol), $100 \mathrm{nM}$ rapamycin (rapa) and with the drug combination as indicated. HSC70 expression levels served as loading control. The data represent the results from one experiment. solv, solvent.

In the ARMS cell line RMS-13 (Figure 29) the effects of the drugs were more heterogeneous than in ERMS cells. Main differences to the ERMS cell lines (Figure 27, Figure 28) was the lack of inhibition of AKT phosphorylation by $\mathrm{HhA}$ and the moderate stimulation of AMPK phosphorylation upon treatment with HhA, PI103, GDC-0941, MK-2206 or everolimus (Figure 29). Furthermore, the incubation with PI103 and GDC-0941 strongly activated caspase 3. Active caspase 3 levels induced by PI103 and GDC-0941 were repressed in combination with HhA compared to single drug treatments. Another difference was that the combination HhA plus MK-2206 repressed pS6/S6 as well as pAMPK/AMPK protein levels. The combination treatment of HhA plus mTOR inhibitors slightly increased LC3-II levels compared to the respective single drug incubations. Similar to the results in ERMS cells, LC3-II levels were enhanced upon treatment with HhA, GDC-0941 and MK-2206. In addition, combination of HhA with mTOR inhibitors decreased pAKT levels compared to the single drug treatments. 


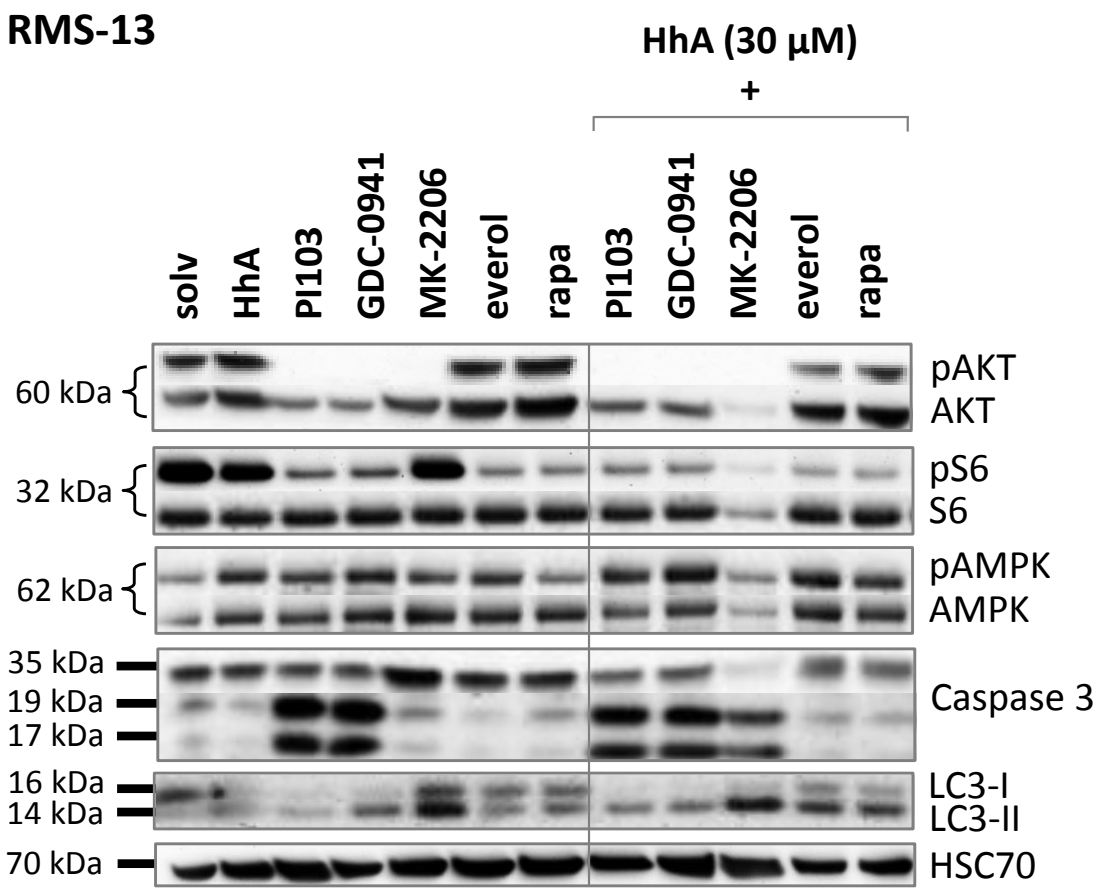

Figure 29: Effects of HhA and/or PI3K/AKT/mTOR inhibitors on PI3K/AKT/mTOR signaling, phosphorylation status of AMPK and LC3-II protein levels in RMS-13 cells. Western blot analysis of RMS-13 cells incubated for $48 \mathrm{~h}$ with $30 \mu \mathrm{M}$ HhA, $3 \mu \mathrm{M}$ PI103, $10 \mu \mathrm{M}$ GDC-0941, $1 \mu \mathrm{M}$ MK-2206, $50 \mathrm{nM}$ everolimus (everol), $100 \mathrm{nM}$ rapamycin (rapa) and with the drug combination as indicated. HSC70 expression levels served as loading control. The data represent the results from one experiment. solv, solvent.

In the ARMS cell line Rh41, HhA stimulated phosphorylation of AKT (Figure 30). Similar effects were seen with both mTOR inhibitors. Single treatment with MK-2206 decreased S6 phosphorylation and increased active caspase 3 in contrast to all other RMS cell lines. LC3-II was induced only by $\mathrm{HhA}$ and MK-2206. As in the other cell lines a reduction of pAKT was observed when combining HhA with mTOR inhibitors. In addition, increase in activated caspase 3 and LC3-II levels mediated by MK-2206 was repressed upon combination with HhA. 


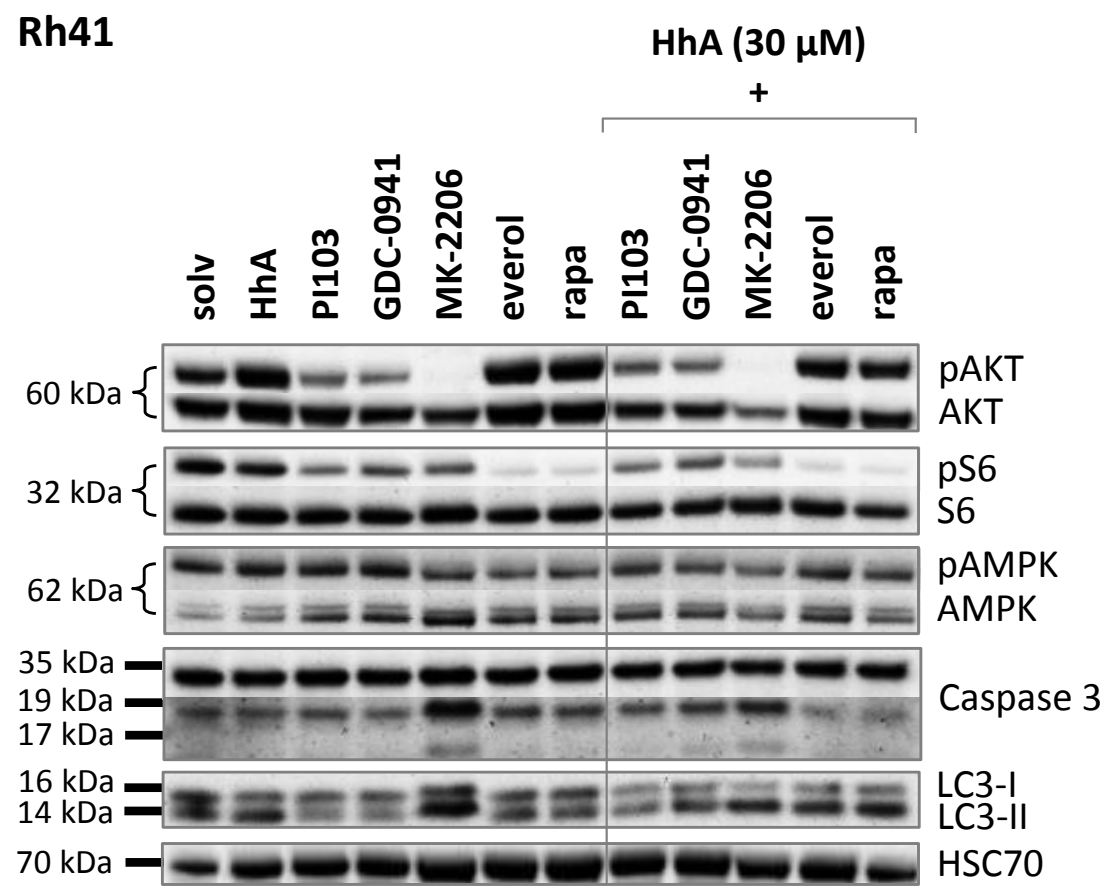

Figure 30: Effects of HhA and/or PI3K/AKT/mTOR inhibitors on PI3K/AKT/mTOR signaling, phosphorylation status of AMPK and LC3-II protein levels Rh41cells. Western blot analysis of Rh41 cells incubated for $48 \mathrm{~h}$ with $30 \mu \mathrm{M}$ HhA, $200 \mathrm{nM}$ PI103, $500 \mathrm{nM}$ GDC-0941, $1 \mu \mathrm{M}$ MK-2206, $50 \mathrm{nM}$ everolimus (everol), $100 \mathrm{nM}$ rapamycin (rapa) and with the drug combination as indicated. HSC70 expression levels served as loading control. The data represent the results from one experiment. solv, solvent.

In summary, the data show that the combination of HhA plus PI3K/AKT/mTOR inhibitors induces variable effects among the analyzed RMS cell lines. In general the effects of the drugs are more heterogeneous in ARMS than in ERMS. Phosphorylation of AKT, which is increased or unaffected upon treatment with everolimus or rapamycin alone, is repressed upon combination with HhA in all RMS cell lines.

Furthermore the combinations of HhA plus PI3K/AKT/mTOR inhibitors reduce pAMPK levels in RD cells, but induce it in RMS-13 and do not alter it in RUCH-2 cells. Cooperative effects on caspase 3 activation are triggered by HhA plus PI103, or HhA plus GDC-0941 in RUCH-2 (Figure 28) and by HhA plus MK-2206 in RD (Figure 27) and RMS-13 (Figure 29). Paradoxically, the combination of HhA plus PI103 or HhA plus GDC-0941 results in a reduction of caspase 3 activity in RMS-13 cells (Figure 29), which is also revealed in Rh41 when HhA was combined with MK-2206 (Figure 30). Finally, the combination of HhA plus PI3K/AKT/mTOR inhibitors further strengthened the increase in LC3-II levels compared to the single drug treatments in ERMS. However, it should be emphasized that the latter results in RUCH-2, RMS-13 and Rh41 are only preliminary. The respective experiments have to be repeated. 


\subsection{In vivo effects of the SMO antagonist HhA plus PI3K inhibitor GDC-0941}

As described in section 6.2, the combination of HhA plus GDC-0941 intensifies several antitumoral effects in human RMS cell lines and results in a cooperative antiproliferative effect (Figure $25 \mathrm{~A}, \mathrm{~B}, \mathrm{D})$. In order to investigate whether these effects also occur in vivo, the drugs were applied to RMS bearing Ptch $^{+/}$mice, which spontaneously develop RMS, that resemble the embryonal subtype in humans. Therefore, these mice are a perfect model for the preclinical evaluation of $\mathrm{HH}$ pathway antagonists and other drugs in the treatment of ERMS.

\subsubsection{Effect of HhA plus GDC-0941 on tumor growth in $\mathrm{Ptch}^{+/-}$mice}

RMS bearing mice were treated orally every day for five weeks with vehicle, $100 \mathrm{mg} / \mathrm{kg} \mathrm{HhA,}$ $75 \mathrm{mg} / \mathrm{kg}$ GDC-0941 or with the combination of both (as described in section 5.4.6). To calculate the tumor growth during treatment, the tumor volume was measured via VCT at the day of therapy onset, two weeks after therapy onset and at therapy end.

As shown in Figure 31, the analysis revealed that in comparison to the control the single drug treatments with either HhA or GDC-0941 significantly inhibited the tumor growth at the end of the treatment. In addition, the data showed that the PI3K inhibitor GDC-0941 was more potent than HhA. Interestingly and similar to the in vitro situation, the combination of both drugs induced a cooperative antitumoral effect, which was already seen after two weeks. The tumor growth was thereby significantly decreased compared to the vehicle controls and to tumors that have been treated with HhA alone (Figure 31).

In summary, the results suggest that HhA is a potential treatment option in RMS, especially in combination with a PI3K inhibitor. 


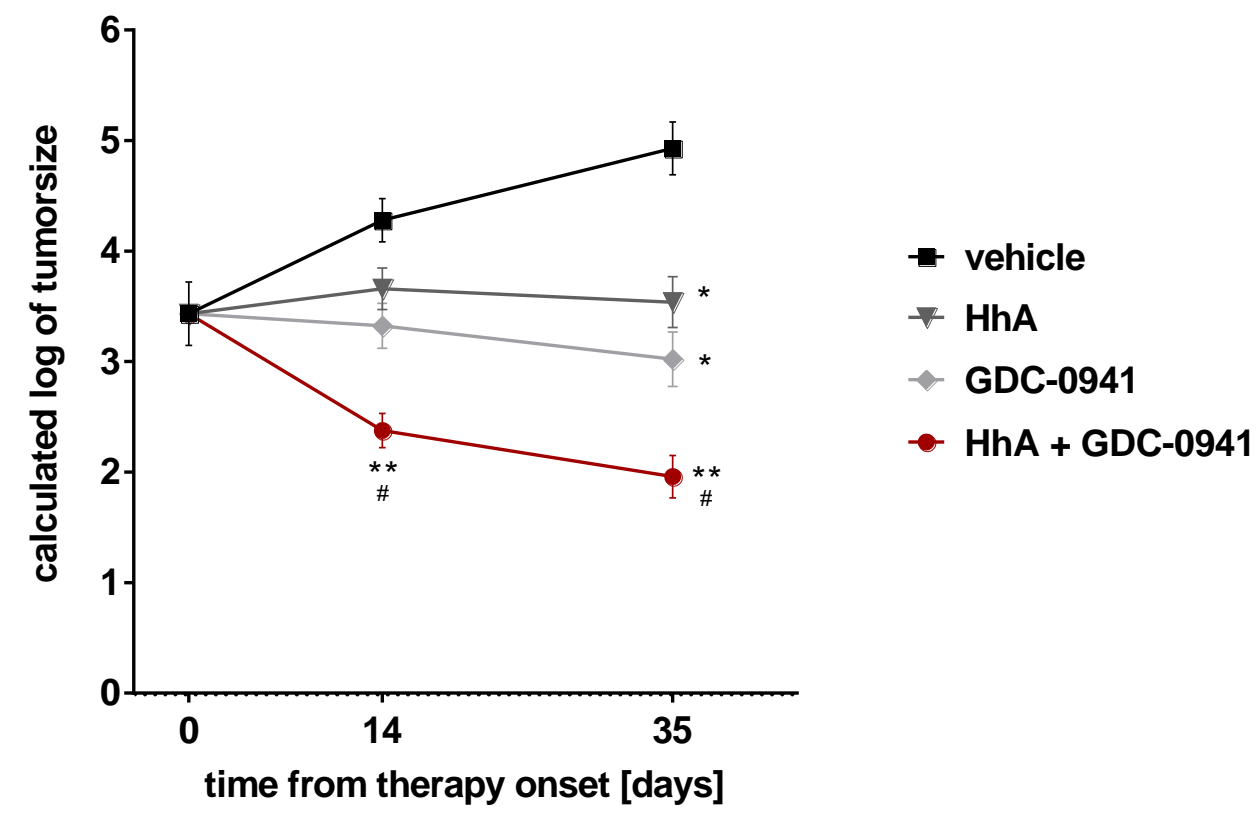

Figure 31: Cooperative antitumoral effect of HhA and/or GDC-0941 on RMS growth in vivo. Ptch ${ }^{+/-}$mice were treated orally for five weeks with a daily dose of $100 \mathrm{mg} / \mathrm{kg}$ HhA (n=6) and $75 \mathrm{mg} / \mathrm{kg}$ GDC-0941 (n=6) alone or in combination $(n=9)$. Vehicle treated animals served as controls $(n=6)$. Tumor size was measured by VCT. *, $\mathrm{p}<0.05 ; * *, \mathrm{p}<0.01$ compared to tumors of vehicle treated mice; \#, $\mathrm{p}<0.05$ compared to tumors of mice treated with HhA. Multiple testing was done by the method of Tukey.

\subsubsection{Immunohistochemical analysis of the proliferation marker Ki67}

In order to analyze the proliferative capacity of the tumors from the four cohorts, paraffin sections were stained with an anti-Ki67 antibody (Figure $32 \mathrm{~A}$ ). The calculation of Ki67 positive cells revealed that single drug treatment with either HhA or GDC-0941 significantly decreased the amount of Ki67 positive cells in the tumors by approximately $75 \%$ compared to the control (Figure $32 \mathrm{~B}$ ). However, the combination of both drugs did not result in a further reduction of Ki67 positive tumor cells. Thus, the cooperative effect of the drugs on tumor growth inhibition must be due to other antitumoral effects, e.g. possibly by induction of apoptosis (see Figure 28 for combination effect on caspase 3 activation in RUCH-2). 
A

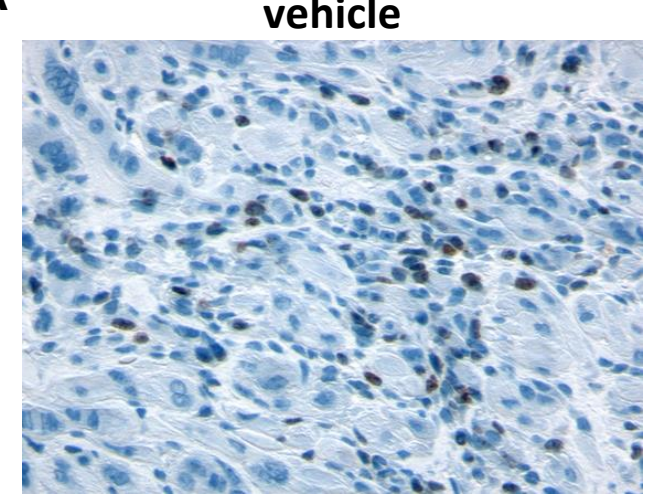

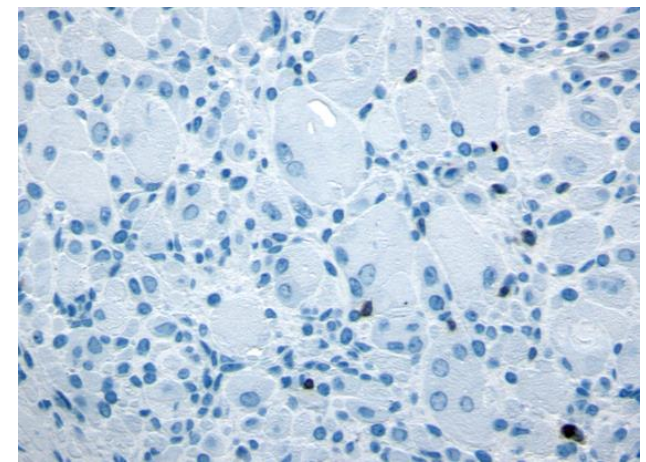

GDC-0941
B

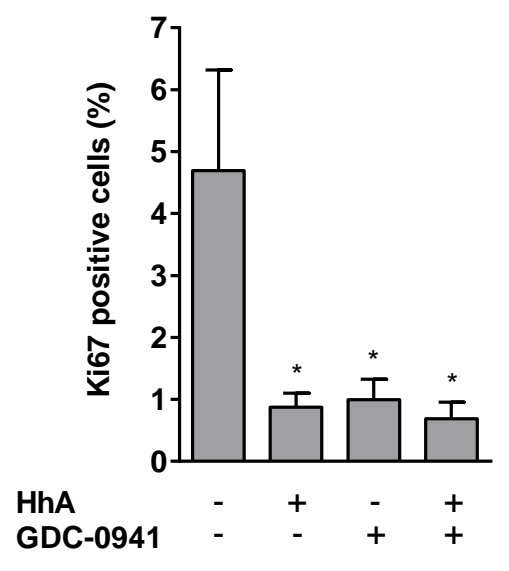

HhA
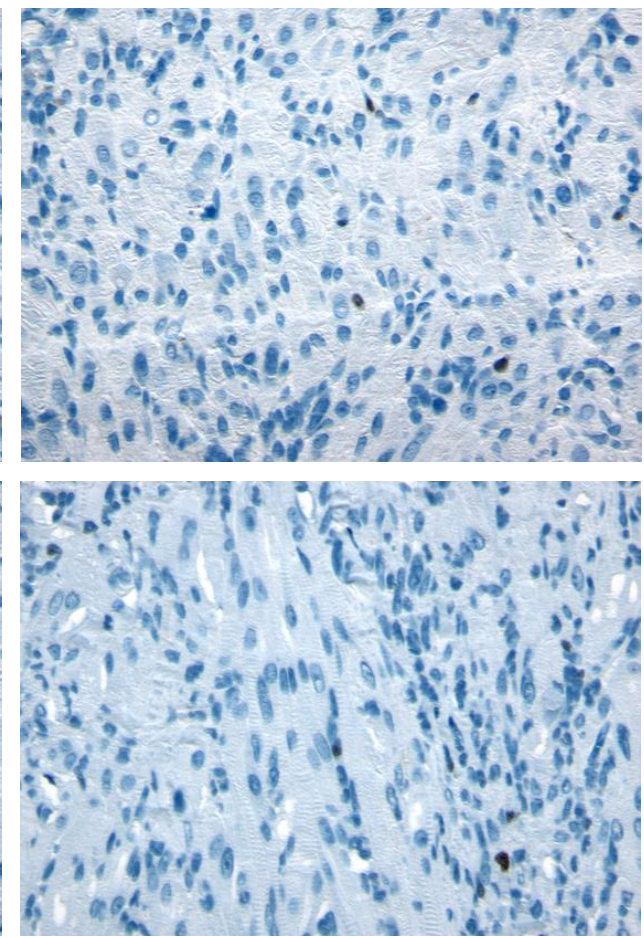

HhA + GDC-0941
C

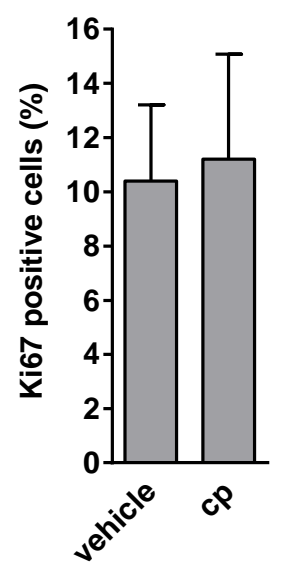

Figure 32: Analysis of the proliferation marker Ki67 in tumors derived from in vivo treatment. Analysis of the proliferation marker Ki67 in tumors derived from in vivo treatment. (A) Ki67 stained paraffin embedded sections of tumors derived from $\mathrm{Ptch}^{+/}$mice treated orally for five weeks with a daily dose of $100 \mathrm{mg} / \mathrm{kg} \mathrm{HhA}(\mathrm{n}=6)$ and $75 \mathrm{mg} / \mathrm{kg}$ GDC-0941 $(\mathrm{n}=6)$ alone or in combination $(\mathrm{n}=9)$. Vehicle treated animals served as controls $(n=6)$. (B) Calculated amount of Ki67 positive cells (in \%) counted on Ki67 stained tumor sections from the in (A) described in vivo treatment. (C) Ki67 positive cells (in \%) of tumors derived from $\mathrm{Ptch}^{+/-}$mice treated systemically over a period of four weeks with a daily dose of $20 \mathrm{mg} / \mathrm{kg}$ cyclopamine $(\mathrm{cp})(\mathrm{n}=3)$ or vehicle $(\mathrm{n}=4)$. The in vivo treatment of the tumors in (C) was done by Ines Ecke as described previously (Ecke et al., 2008). The data represented mean +/$\mathrm{SEM} ;{ }^{*}, \mathrm{p}<0.05$ compared to the vehicle treatment. 


\section{RESULTS}

Ki67 stainings were also done on tumor samples of mice that have been treated with cyclopamine (Figure 32 C). As described by Ines Ecke from our group (Ecke et al., 2008), tumor bearing $\mathrm{Ptch}^{+/}$mice were treated systemically over four weeks with a daily dose of $20 \mathrm{mg} / \mathrm{kg}$ cyclopamine or vehicle. However, in this cohort the tumor volume was not reduced by cyclopamine treatment. Instead the tumor growth rather seemed to be increased compared to the vehicle treated mice (Ecke et al., 2008). These data indicate that in contrast to HhA and/or GDC-0941, cyclopamine does not exert any antiproliferative effect in vivo.

Indeed, the analysis of the proliferation marker Ki67 in the tumor samples revealed, that cyclopamine does not reduce the number of Ki67 positive cells (Figure $32 \mathrm{C}$ ). This is in line with the in vitro data of this thesis, showing that cyclopamine either did not inhibit cellular proliferation (Figure $10 \mathrm{~A}, \mathrm{C}$ ) or induced it (Figure $10 \mathrm{~B}, \mathrm{D}$ ).

Because the effective tumor growth inhibition upon HhA and/or GDC-0941 treatment was not associated with a decrease in Ki67 positive cells, it was intended to analyze the activity of $\mathrm{PI} 3 \mathrm{~K} / \mathrm{Akt} / \mathrm{mTOR}$ signaling and of Ampk and the expression of Lc3-II in the tumor samples. Unfortunately, the samples that have been treated with HhA and/or GDC-0941 were too small for RNA or protein isolation. Hence, in an alternative approach, cultured primary RMS cells isolated from $\mathrm{Ptch}^{+/-}$mice were incubated with the substances in vitro.

The findings revealed that single drug treatment with HhA reduced phosphorylation of Akt and slightly induced Lc3-II compared to the control (Figure 33). As expected, GDC-0941 also inhibited phosphorylation of Akt. Moreover GDC-0941 decreased phosphorylation of S6 and Ampk and slightly activated caspase 3. Furthermore, it increased the Lc3-II protein level, which was higher compared to that induced by HhA. When HhA and GDC-0941 were combined, the effects on the respective proteins were not different compared to GDC-0941, except for caspase 3 activation, which was slightly increased upon combination treatment. However, these finding are the results from one experiment and the western blot has to be repeated in the future. 


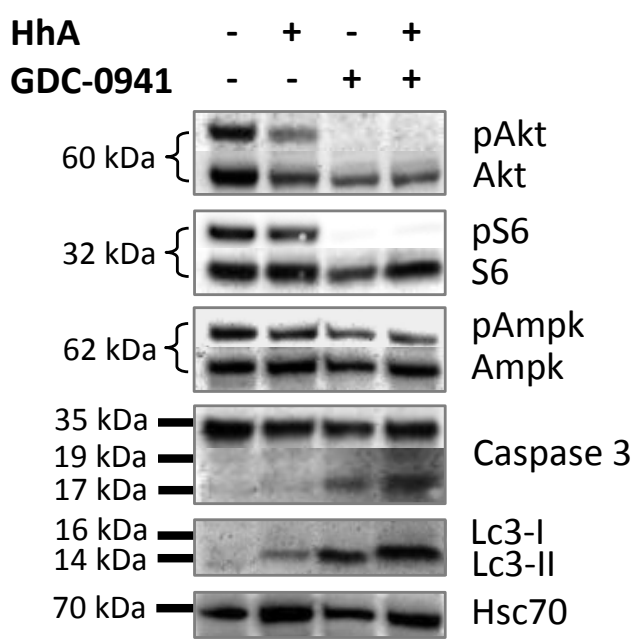

Figure 33: Effects of HhA and/or GDC-0941 on PI3K/Akt/mTOR signaling, activation of Ampk and Lc3-II protein levels in murine primary RMS cells. Western blot analysis of primary cells, which were isolated from RMS bearing $\mathrm{Ptch}^{+-}$mice and incubated for $24 \mathrm{~h}$ with $10 \mu \mathrm{M}$ HhA and/or $10 \mu \mathrm{M}$ GDC-0941. Hsc70 expression levels served as loading control. The data represent the results from one experiment. 


\section{DISCUSSION}

\subsection{Effects of four SMO antagonists on RMS cell lines}

The HH signaling pathway regulates cellular proliferation, differentiation and tissue patterning. Therefore, it is not surprising that a pathological activation of $\mathrm{HH}$ signaling can result in cancer development. Types of cancer which show aberrant HH pathway activation include malignancies of the skin, brain, prostate, lung, breast and also subgroups of RMS (Pressey et al., 2011; Zibat et al., 2010). Thus, the HH pathway is considered to represent a new therapy option and RMS might be sensitive towards a targeted therapy using small molecule inhibitors directed against components of the HH pathway.

In the last years, various HH inhibitors - predominantly targeting SMO - have been developed. The efficacy of these SMO antagonists has been investigated already in several clinical trials (Amakye et al., 2013). GDC-0449 as the frontrunner of these drugs, has already been approved in 2012 for advanced and metastasizing BCC (Rudin, 2012) and LDE225, as another potent SMO inhibitor from a novel structural class (Pan et al., 2010), was approved 2015 in the USA for treatment of advanced BCC. HhA is the forerunner of GDC-0449 and provided convincing responses of MB in mouse models (Romer et al., 2004). Cyclopamine represents the natural prototype of SMO inhibitors (Taipale et al., 2000).

In the first part of this thesis, the effects of these four SMO antagonists on RMS cells were investigated in order to analyze potential antitumoral effects in vitro. In order to include the most common subtypes of RMS, the effects were analyzed in the ERMS cell lines RD and RUCH-2 and in the ARMS cell lines RMS-13 and Rh41.

\subsubsection{Effects on HH signaling activity}

As already mentioned, the used RMS cell lines express major components of the HH signaling pathway, including the main target genes GLII and PTCH (Graab et al., 2015; Ridzewski et al., 2015). Therefore, these cell lines show activation of the HH signaling pathway. In order to investigate if GDC-0449, LDE225, HhA and cyclopamine were able to inhibit HH signaling in RMS cells, HH pathway activity was analyzed by qRT-PCR of GLII, because GLII mRNA expression is the most reliable indicator for active $\mathrm{HH}$ signaling (reviewed in (Scales and de Sauvage, 2009)). The data revealed that intrinsic HH pathway activation in RMS cell lines was modulated by treatment with SMO antagonists, although the effects were dependent on the 
applied drug and their respective concentration, and varied from cell line to cell line (Figure 5). Against all expectations, an inhibition of GLII expression by SMO inhibitors was in general not prior-ranking in RMS cell lines and frequently the drugs had no effect on GLII expression (e.g. $1 \mu \mathrm{M}$ GDC-0449, LDE225 and HhA in RUCH-2 cells). This has also been described for GDC-0449 in RMS cells by other groups (Graab et al., 2015). Conversely, GLII expression was also not modulated in RD cells after treatment with the SMO agonist SAG (Figure 15 B). However mutations in SMO, which potentially could impede binding of the drugs to SMO and consequently impair their efficacy in the analyzed RMS cell lines were excluded (personal communication Javed Khan, National Cancer Institute, Bethesda, USA). These findings indicate that canonical HH signaling via the SMO/GLI axis is not the main source of $\mathrm{HH}$ pathway activation and accordingly of GLII expression in RMS cells. Thus, GLII expression in RMS cells is rather regulated independently of SMO by a noncanonical mechanism. This assumption is also supported by data showing that knockdown of SMO has no effect on proliferation of ERMS cell lines and that inhibition of GLI1/2 by the GLI inhibitor GANT61 induces apoptosis, while cyclopamine does not (Tostar et al., 2010).

As mentioned in section 2.3.3, noncanonical activation of $\mathrm{HH}$ signaling has been described in several cancers. One of the pathways that can activate noncanonical $\mathrm{HH}$ signaling is PI3K/AKT/mTOR signaling (Filbin et al., 2013; Stecca et al., 2007; Wang et al., 2012). Activation of the PI3K/AKT/mTOR pathway often appears in RMS (Petricoin et al., 2007; Renshaw et al., 2013) and is also observed in the analyzed RMS cell lines (as demonstrated by distinct expression of pAKT and pS6 in all RMS cell lines; e.g. see Figure 13 solvent treated cells). Indeed, inhibition of this pathway with PI3K/AKT/mTOR inhibitors efficiently decreased GLII expression in the used RMS cell lines (Figure 21), especially obvious in RD and RUCH-2 cells (Figure $21 \mathrm{~A}, \mathrm{~B}$ ). Inhibition of $\mathrm{HH}$ signaling in RMS in vitro and in vivo after treatment with PI3K/AKT/mTOR inhibitors has also been shown by other groups. For instance, PI103 reduces GLII transcription in RD cells (Graab et al., 2015) confirming the results of this thesis. Furthermore the mTOR inhibitor rapamycin decreases GLI1 mRNA as well as GLI1 protein levels in RMS xenografts (Kaylani et al., 2013). These findings strongly suggest that in the analyzed RMS cell lines, GLII transcription and thus the HH pathway activity can be regulated through a noncanonical mechanism independently of SMO, which probably involves $\mathrm{PI} 3 \mathrm{~K} / \mathrm{AKT} / \mathrm{mTOR}$ signaling.

On the other hand, it is likely that the PI3K/AKT/mTOR pathway is regulated by SMO. Thus, as shown in this thesis, HhA decreases AKT activity in ERMS cells (Figure 13 A, B). Since the $\mathrm{PI} 3 \mathrm{~K} / \mathrm{AKT} / \mathrm{mTOR}$ pathway regulates GLII transcription (as demonstrated in this thesis and also 


\section{DISCUSSION}

reported by other groups (Rao et al., 2004; Riobo et al., 2006; Wang et al., 2012)), reduced AKT activity subsequently could repress GLII expression in these cell lines (Figure 5 RD, RUCH-2). This scenario could also explain the induction in GLII transcription upon cyclopamine treatment in most cases (Figure 5), because (in contrast to HhA) cyclopamine increased AKT activity in all cell lines (Figure 13). Notably, the effect on AKT is not regulated by GLI factors themselves because GLI1/2 inhibition by GANT61 does not influence pAKT levels in RMS cells (Graab et al., 2015). Therefore, interaction between both pathways might be due to a SMO/AKT/GLI1 axis. However, this is not consistently seen for all settings and therefore needs verification in the future. The data also indicate that the noncanonical SMO derived effects on PI3K/AKT/mTOR pathway were regulated differently by SMO inhibitors, e.g. HhA inhibited AKT but cyclopamine induced AKT activity in most cell lines, whereas GDC-0449 and LDE225 showed no effect on AKT activity.

Besides cyclopamine, also other SMO inhibitors paradoxically can stimulate GLII expression levels in some settings (e.g. LDE225 in RMS-13 cells). The possibility that the drugs modulate other signaling components, which vice versa could activate GLII expression, has been demonstrated for the SMO agonist SAG. Thus, SAG induced HH signaling activity in a dose dependent manner with an EC50 of $3 \mathrm{nM}$, but it paradoxically inhibited the activity at concentrations above $1 \mu \mathrm{M}$ (Chen et al., 2002b). The authors suggested a model, in which SAG may interact not only with SMO, but also with another cellular effector of SMO. According to their model, optimal SAG concentrations induce $\mathrm{HH}$ pathway activation by enabling the association of SMO with the respective cellular effector. Higher SAG concentrations however would begin to inhibit this process, as SAG then would independently bind both, SMO and the effector (Chen et al., 2002b). Comparable models might apply to the SMO inhibitors used in this study and could explain some concentration dependent effects as well as the triphasic GLII expression patterns in RD cells (i.e. a decrease at $10 \mu \mathrm{M}$, a re-increase at $30 \mu \mathrm{M}$ compared to $10 \mu \mathrm{M}$ and again a decrease at $50 \mu \mathrm{M}$ upon GDC-0449, LDE225 or HhA treatment; Figure 5).

Igf 2 is another downstream target of Hh signaling and is essential for the formation of Ptch associated RMS in $\mathrm{Ptch}^{+/}$mice (Hahn et al., 2000). Moreover, IGF2 is a very important embryonal growth factor and plays a crucial role in RMS pathology (Martins et al., 2011). Indeed, most human RMS show high IGF2 gene expression levels (de Souza et al., 2014; ElBadry et al., 1990; Zhan et al., 1994). Therefore, it was hypothezised that repression of IGF2 mediated by SMO inhibitors could further strengthen the antitumoral effects of these drugs in RMS. In order to analyze the impact of SMO antagonists on IGF2, its expression was 
determined by qRT-PCR. The investigation revealed that SMO inhibitors also modulated IGF2 transcription in human RMS cell lines. Similar to modulation of GLII expression, this differed from drug to drug, their concentrations and from cell line to cell line (Figure 6).

Interestingly, modulation of $I G F 2$ expression was not necessarily correlated with the corresponding GLII expression (compare Figure 6 with Figure 5). Similar to GLII, inhibition of $I G F 2$ expression by SMO inhibitors was in general not pronounced in RMS cell lines. This suggests that the SMO inhibitors regulate IGF2 expression probably by noncanonical mechanisms or by regulation of other factors that are involved in regulation of IGF2 mRNA transcription. For instance, the transcription factor AP-2, which mediates responses of cyclic adenosine monophosphate (cAMP) dependent protein kinase A (PKA) and protein kinase C (PKC), can increase IGF2 transcription in RMS cell lines (Zhang et al., 1998b). In addition, p53 is associated with repression of IGF2 transcription in RMS cell lines (Zhang et al., 1998a). However, the precise mechanisms of p53 mediated regulation of IGF2 transcription remained not fully elucidated (Martins et al., 2011).

Remarkably, it has been shown that IGF2 functions as an autocrine growth factor in RMS cells (El-Badry et al., 1990; Minniti et al., 1994) and induces phosphorylation of AKT in cerebellar granule cell precursors and MB cells (Hartmann et al., 2005). Therefore, it is possible that IGF2 (via its receptor IGF1R) signals back to the cell in an autocrine manner and activates the PI3K/AKT/mTOR pathway. This might be the case e.g. for 5 and $10 \mu \mathrm{M}$ cyclopamine that induced IGF2 expression in RMS-13 cells (Figure 6), which correlated with activation of AKT (Figure $13 \mathrm{C}$ ). Vice versa 10 and $30 \mu \mathrm{M}$ HhA repressed IGF2 transcription in RD cells (Figure 6), which was associated with reduced pAKT levels (Figure 13 A) and also with decreased cellular proliferation (Figure $10 \mathrm{~A}$ ). Moreover, the correlation between IGF2 and AKT was also be revealed on protein level. In RD cells $10 \mu \mathrm{M}$ HhA reduced IGF2 and pAKT protein levels, $100 \mathrm{nM}$ SAG increased IGF2 and pAKT, and HhA plus SAG decreased IGF2 and pAKT protein expression (Figure 15 A). However, the relation between IGF2 levels and pAKT levels was not consistently observed, which was probably due to cell line specific differences or heterogeneous drug effects. For instance in Rh41 cells, 10 and $30 \mu \mathrm{M}$ GDC-0449 increased IGF2 expression (Figure 6) and stimulated cell proliferation (Figure $10 \mathrm{D}$ ), but AKT levels were not modulated (Figure 13 D). Because IGF1R stimulation can also activate the RAS/MEK/ERK cascade (Pollak, 2008), it is possible that the increase in IGF2 may have activated the RAS/MEK/ERK pathway which subsequently stimulated cellular proliferation of Rh41 upon 10 and $30 \mu \mathrm{M}$ GDC-0449 treatment. Thus, similar to GLI1, the data suggest that IGF2 transcription might be modulated by the drugs in a noncanonical manner. 


\section{DISCUSSION}

\subsubsection{Effects on muscle differentiation}

It has been demonstrated that $\mathrm{HH}$ signaling also regulates the expression of muscle differentiation markers. For instance, MYOD expression is suppressed by GLIs in RMS cells (Gerber et al., 2006). In addition, HH signaling inhibits terminal muscle differentiation (BrenMattison and Olwin, 2002). Therefore, it was hypothesized that inhibition of $\mathrm{HH}$ signaling by SMO antagonists should lead to induction of $M Y O D$ and $M Y H 1$ expression and thus, to a more differentiated RMS phenotype.

However, a negative correlation between GLII transcription and expression of muscle differentiation markers, as described in the literature, was not continuously observed. The data demonstrate that MYOD and MYHI expression was only induced by some SMO inhibitors at certain concentrations in specific cells lines (e.g. GDC-0449 in Rh41 cells; Figure 7, Figure 8), whereas other inhibitors rather led to a downregulation of $M Y O D$ and $M Y H 1$ gene expression (e.g. LDE225 and HhA in RD cells; Figure 7, Figure 8). Based on the observation that GLII expression can be regulated in a noncanonical manner in the analyzed RMS cell lines, it is not surprising that SMO inhibitors did also not induce MYOD and MYH1 expression in most settings (compare Figure 5 with Figure 7 and Figure 8). Furthermore, with the exception of Rh41 cells, the $M Y O D$ and $M Y H 1$ expression patterns did not necessarily correspond to each other (compare Figure 7 with Figure 8).

Nevertheless, the expression of the muscle differentiation markers was sometimes associated with IGF2 expression, e.g. HhA treated RD and RMS-13 cells showed a positive correlation between MYH1 and IGF2 levels (compare Figure 8 with Figure 6). Moreover, a positive correlation between both muscle differentiation markers and $I G F 2$ expression was demonstrated also for GDC-0449 and LDE225 in Rh41 cells for mainly all concentrations (compare Figure 7 and Figure 8 with Figure 6). A similar correlation has also been described by other groups. Thus, MyoD induced myocyte differentiation goes along with $\operatorname{Igf} 2$ transcription that is followed by autocrine signaling leading to activation of Akt (Wilson et al., 2003). Even if increased levels of AKT upon treatment with GDC-0449 and LDE225 in Rh41 were not observed (Figure 13 D), a general consequence of autocrine activation of IGF1R signaling by IGF2, i.e. the induction of proliferation, was indeed revealed for GDC-0449 and $10 \mu \mathrm{M}$ LDE225 in this cell line (Figure 10 D).

Heterogeneous effects of SMO modulators on muscle differentiation independently of GLII have also been described by others. For instance, the classical SMO agonist SAG induced GLII transcription and differentiation of mesenchymal C3H10T1/2 cells into osteoblasts, whereas the 
novel SMO agonist GSA-10 stimulated SMO to induce differentiation but in contrast decreased GLII expression (Gorojankina et al., 2013). Moreover, the same authors demonstrated that GDC-0449 and LDE225 blocked GSA-10 induced differentiation. This is similar to data presented in this thesis showing that LDE225 and HhA inhibited expression of myogenic markers in RD cells (Figure 7, Figure 8). Finally, Gorojankina et al. confirmed heterogeneous effects of SMO inhibitors on differentiation, in which LDE225 potentially inhibited GSA-10 induced differentiation, whereas GDC-0449 was less effective and cyclopamine did not impact on differentiation at all (Gorojankina et al., 2013).

\subsubsection{Effects on cellular proliferation and apoptosis}

Antiproliferative and proapoptotic effects were mainly triggered by LDE225 and HhA in most of the RMS cell lines (Figure 10, Figure 12). The decrease of the proliferation rate obtained by 10 and $30 \mu \mathrm{M}$ HhA in RD and RUCH-2 cells (Figure $10 \mathrm{~A}, \mathrm{~B}$ ) and the increase of apoptosis by $30 \mu \mathrm{M}$ HhA in RD cells (Figure $12 \mathrm{~A}$ ) was additionally associated with the repression of GLI1 and IGF2 expression (Figure 5, Figure 6) and with reduction of AKT activity (Figure 13 A, B). However, a similar association was not seen for most of the other inhibitors and cell lines.

As described in 2.3.3 noncanonical HH signaling derived from SMO can control different cellular processes independently of GLI transcription factors (reviewed in (Robbins et al., 2012)). This noncanonical activity of SMO could explain the observed antitumoral effects of SMO antagonists (e.g. decrease of proliferation and induction of apoptosis) and that this was not necessarily correlated with GLII inhibition. For instance, $30 \mu \mathrm{M}$ LDE225 and HhA induced antiproliferative and proapoptotic effects in RMS-13 cells, but did not alter GLII expression or moderately induced it, respectively (compare Figure $10 \mathrm{C}$ and Figure $12 \mathrm{~B}$ with Figure 5). Conversely inhibition of $\mathrm{HH}$ signaling was detected for $10 \mu \mathrm{M}$ GDC-0449 in RD cells (Figure 5 ), but resulted in a stimulation of proliferation (Figure $10 \mathrm{~A}$ ). Similar to that inhibition of IGF2 expression did not necessarily correlate with decreased proliferation. The lack of correlation between growth inhibition and decreased HH pathway activity (measured by GLII and PTCH expression) is also described in the literature and was detected in a large panel of human cancer cell lines upon treatment with HhA and cyclopamine (Yauch et al., 2008).

GDC-0449 and cyclopamine induced proliferation in some RMS cells at certain concentrations (e.g. $10 \mu \mathrm{M}$ GDC-0449, $5 \mu \mathrm{M}$ cyclopamine in RUCH-2 and Rh41 cells; Figure $10 \mathrm{~B}, \mathrm{D}$ ). Moreover, they did not induce apoptosis in most of the cell lines (Figure 12), which has been also demonstrated by other groups for ERMS cell lines treated with cyclopamine (Tostar et al., 


\section{DISCUSSION}

2010). In general, the increase in BrdU incorporation upon GDC-0449 and cyclopamine treatment was neither correlated with induction of GLII expression nor IGF2 overexpression. However, in some settings increased proliferation and lack of apoptosis was accompanied by increased pAKT levels. This was e.g. seen after cyclopamine treatment of RUCH-2 and Rh41 cells (Figure 10 B, D). Thus, activation of AKT could be the underlying mechanism responsible for increased cellular proliferation and lack of apoptosis. Indeed, previous work from our lab showed that treatment of RMS bearing $\mathrm{Ptch}^{+/}$mice with cyclopamine did not inhibit tumor growth but rather increased it (Ecke et al., 2008). Whether this growth induction by cyclopamine is also associated with increased AKT phosphorylation remains to be established in the future.

Of course it is possible that off-target effects, which could be derived from the varying molecular structure of the drugs, could account for their heterogeneous effects on proliferation and apoptosis. Such effects have to be excluded in future experiments (see section 7.3).

However despite all these puzzling results, the data presented here suggest that LDE225 and HhA are the most potent drugs with respect to their antiproliferative and proapoptotic capacity. This is very important when using the drugs in RMS therapy knowing that SMO inhibitors can rapidly lead to resistance (see 2.5.4). For instance, GDC-0449 resistance occurred in MB through a mutation in SMO that impedes GDC-0449 binding while keeping HH signaling active (Rudin et al., 2009; Yauch et al., 2009). Similar resistance mechanisms have been described for LDE225 (Buonamici et al., 2010). Due to these facts, the selection of appropriate SMO inhibitors that instantly and very potently inhibit proliferation and/or induce apoptosis is of uttermost importance for the clinics.

\subsubsection{Effects on PI3K/AKT/mTOR signaling, on AMPK activity and on LC3-II expression}

As already mentioned, $\mathrm{HH}$ signaling can modulate the activation status of $\mathrm{PI} 3 \mathrm{~K} / \mathrm{AKT} / \mathrm{mTOR}$ signaling and vice versa AKT can stabilize and thus activate GLI transcription factors (Fu et al., 2006; Morton et al., 2007; Riobo et al., 2006; Wang et al., 2012). Our data demonstrate that HhA inhibits phosphorylation of AKT in both ERMS cell lines (Figure $13 \mathrm{~A}, \mathrm{~B}$ ), whereas the other SMO antagonists do not (Figure 13). In contrast, cyclopamine rather enhances pAKT levels (Figure 13). Effects on S6 phosphorylation were not observed. This suggests indeed a crosstalk between $\mathrm{HH}$ and AKT signaling. However, as with many of the parameters described before, this crosstalk was restricted to certain drugs, characterized by either inhibition or activation of AKT 
and/or was cell line dependent. In the case of HhA it is likely that the reduction of pAKT levels contributed to the very effective antitumoral properties of this drug.

SMO antagonists can also activate a noncanonical SMO/Ca ${ }^{2+} / \mathrm{AMPK}$ cascade, which triggers a Warburg like effect (Teperino et al., 2012). This has been demonstrated for GDC-0449 and cyclopamine, but not for LDE225 in cell culture. Furthermore, the physiological outcome of $\mathrm{Ca}^{2+}$ influx and AMPK mediated catabolism may be responsible for the two major side effects of SMO inhibitors, such as muscle cramping and weight loss (Teperino et al., 2012). Indeed, pAMPK levels were upregulated in e.g. Rh41 cells by all SMO inhibitors (Figure 13 D). This suggests that also HhA and LDE225 can induce a Warburg like effect. However, the regulation of pAMPK strongly depended on the drug concentration and the cellular context. Hence, it is difficult to draw valid conclusions about the modulation of pAMPK by SMO antagonists in RMS.

Because HH signaling can inhibit autophagosome synthesis (Jimenez-Sanchez et al., 2012), it was also investigated whether the applied drugs were able to induce autophagy in RMS cells. Indeed LDE225, HhA and cyclopamine strongly increased the levels of LC3-II in nearly all settings (Figure 13). In contrast, GDC-0449 was not able to induce autophagy in any of the used cell lines, which is in line with data from hepatocellular carcinoma cells (Wang et al., 2013). Induction of autophagy upon inhibition of the HH pathway has been shown in a variety of other cancer cells. In pancreatic carcinoma cells, that were treated with GLI inhibitor GANT61, the induced autophagy was accompanied with reduced cell viability and increased apoptosis in vivo and in vitro (Wang et al., 2013; Xu et al., 2014). This is different from the data presented here, which showed that induction of autophagy by SMO inhibitors is not necessarily accompanied by antiproliferative or proapoptotic effects. For instance cyclopamine treatment of Rh41 cells increased LC3-II levels but induced proliferation without affecting apoptosis (compare Figure 13 D with Figure 10 D and Figure 12 C). Furthermore, induction of autophagy by LDE225, HhA and cyclopamine was independent from GLII expression (compare Figure 13 with Figure 5). Similar observations have been made by other groups, e.g. LC3-II levels were increased by cyclopamine in Gli2 null mouse embryonic fibroblasts (Jimenez-Sanchez et al., 2012). Moreover, the SMO agonist SAG $(1 \mu \mathrm{M})$ induced LC3-II levels instead of blocking it (Figure 15 A). SAG also did not modulate GLII expression (Figure 15 B) confirming GLI independent modulation of autophagy. These findings suggest that SMO probably regulates autophagy independent of canonical HH signaling in RMS cell lines. 


\subsubsection{Overview on diverse and noncanonical effects of SMO inhibitors in RMS cells}

The efficacy of SMO inhibitors in cancer have been investigated in many preclinical studies. In tumors that show canonical $\mathrm{HH}$ signaling activity due to activating mutations of the $\mathrm{HH}$ pathway (e.g. BCC and MB), SMO inhibitors are effective in inhibition of $\mathrm{HH}$ signaling, repression of proliferation and induction of apoptosis (Amakye et al., 2013; Ng and Curran, 2011).

However, this might be different in RMS in which the HH pathway apparently is activated in a noncanonical manner, i.e. by a SMO independent GLI1 regulation, which is demonstrated in this work and also by other groups (Graab et al., 2015; Tostar et al., 2010). As shown in this thesis, noncanonical regulation of GLII expression in RMS involves the PI3K/AKT/mTOR pathway. Therefore it is not astonishing, that some of the used SMO inhibitors had no antitumoral effects on RMS cell lines or that they were less effective. Similar results have been obtained by other groups who targeted SMO and GLI factors in RMS cell lines. Thus, it has been shown that genetic silencing of GLII/2 or treatment with GANT61 was more efficient in RMS cell lines than genetic silencing of SMO or treatment with GDC-0449 or cyclopamine (Graab et al., 2015; Tostar et al., 2010).

Nevertheless, the results of this thesis clearly demonstrate that some SMO inhibitors i.e. HhA and LDE225 had excellent antitumoral properties on RMS cell lines, although this was sometimes independent from GLII expression and therefore from canonical $\mathrm{HH}$ signaling. Indeed, SMO can regulate several cellular pathways independently of GLI (reviewed in (Robbins et al., 2012)). SMO is a functional G-protein-coupled receptor (GPCR) with selectivity toward heterotrimeric $G_{i}$ proteins and has been shown to mediate noncanonical $\mathrm{HH}$ signaling through the activation of small GTPases (reviewed in (Brennan et al., 2012)). For instance, SMO dependent cell tubulogenesis in endothelial cells was mediated by the small GTPase RhoA, but was not associated with Glil transcription (Chinchilla et al., 2010). Another example for noncanonical signaling of SMO is the observation that Smo promoted fibroblast migration via a downstream $\mathrm{G}_{\mathrm{i}} / \mathrm{PI} 3 \mathrm{~K} / \mathrm{Akt}$ axis, which was also independent of Glil transcription (Polizio et al., 2011). The latter findings also confirm the data of this thesis that SMO can regulate PI3K/AKT signaling. Thus, the antitumoral effects of SMO inhibitors in RMS might be due to the mentioned mechanisms or could be due to other - still unidentified - cellular targets or interaction partners of SMO. Indeed, the results of this thesis indicate that at least HhA inhibits AKT activity in ERMS cells, which could be involved in the excellent antitumoral properties of this drug. 
However, off-target effects of the inhibitors can generally not be excluded and together with cell context specific findings might reflect some inconsistency in the results.

A second important observation is that the four SMO inhibitors used in this thesis had highly different and heterogeneous effects on the analyzed parameters. Similar variances in action of SMO inhibitors have been demonstrated by other groups (Chinchilla et al., 2010; Gorojankina et al., 2013; Teperino et al., 2012; Yam et al., 2009) and indicate that pharmacological modulation of SMO seems to exert complex cellular effects.

This could be due to the fact that SMO inhibitors can stabilize multiple conformations of SMO which confer different SMO activation states and subcellular localizations (reviewed in (Ruat et al., 2014)). In detail, activation of SMO requires two steps: the initial translocation of SMO to the primary cilia followed by a still unidentified activation step of SMO, which allows SMO to trigger the downstream HH signaling cascade (Rohatgi et al., 2009). Therefore SMO can exist in various inactive and active conformations and several SMO antagonists induce these different conformations and favor specific subcellular localizations. Whereas GDC-0449, LDE225 and HhA bind to an inactive form of SMO and prevent its translocation to the cilia, cyclopamine promotes the enrichment of an inactive SMO conformation in the primary cilia (Dijkgraaf et al., 2011; Rohatgi et al., 2009; Wilson et al., 2009; Wu et al., 2012). All this different mode of action at SMO could explain some of the diverse effects of the inhibitors used in this study.

Another explanation for the different actions of the drugs could be due to their different binding modes inside the binding pocket of the SMO transmembrane domain. This could subsequently cause different conformations of SMO and hence diverse cellular effects. They can bind to the same cavity but interacting with different or overlapping residues, e.g. GDC-0449 and cyclopamine mostly bind to the extracellular loops and others like SANT-1 primarily contact the transmembrane residues deeply in the binding pocket (reviewed in (Ruat et al., 2014)). Notably cyclopamine, SANT-1, GDC-0449 and SAG bind to the same binding pocket in the transmembrane domain of SMO (Chen et al., 2002a; Chen et al., 2002b; Wang et al., 2014), but have different cellular functions, i.e. whereas SAG is a SMO agonist, the other drugs are all SMO antagonists. Another possibility might be a binding of the drugs to additional specific molecules that cooperate with SMO in canonical or noncanonical signal transduction.

In summary, the HH pathway in human RMS cell lines is apparently activated in a noncanonical manner, probably by the PI3K/AKT/mTOR pathway. Nevertheless, the SMO antagonists HhA and LDE225 showed excellent antitumoral effects on most of the used RMS cell lines, making them candidates for RMS therapy. In addition, the antitumoral effects of HhA most likely 


\section{DISCUSSION}

involved inhibition of AKT. Due to the highly heterogeneous effects of SMO inhibitors in different RMS cell lines, the use of these drugs in the clinics certainly will require a thorough pretesting before applying them to RMS patients.

\subsection{Effects of combining SMO antagonists with PI3K/AKT/mTOR inhibitors in RMS}

The PI3K/AKT/mTOR pathway is often activated in human RMS (Petricoin et al., 2007; Renshaw et al., 2013). Due to the potential interaction between the HH pathway and the $\mathrm{PI} 3 \mathrm{~K} / \mathrm{AKT} / \mathrm{mTOR}$ signaling cascade in RMS and concerning that some SMO inhibitors i.e. HhA inhibited at least activation of AKT (see Figure 13 A, B), SMO antagonists were combined with $\mathrm{PI} 3 \mathrm{~K} / \mathrm{AKT} / \mathrm{mTOR}$ inhibitors and potential cooperative antitumoral effects were investigated. In order to block PI3K/AKT/mTOR signaling the dual PI3K/mTOR inhibitor PI103, the specific PI3K inhibitor GDC-0941, the pure AKT inhibitor MK-2206 and the mTOR inhibitors everolimus and rapamycin were applied (see Figure 4).

It was hypothesized that the combination of SMO inhibitors with inhibitors of the $\mathrm{PI} 3 \mathrm{~K} / \mathrm{AKT} / \mathrm{mTOR}$ pathway not only improve the anticancer effects, but also may help to potentially lower the dose of each drug and subsequently to avoid side effects. Indeed, SMO inhibitors are known to cause chronic side effects such as weight loss and anorexia in humans as well as bone defects in young mice (Kimura et al., 2008), which may hamper their use especially in children. Additionally, the occurrence of acquired resistance to SMO antagonists further impairs their application. Mechanisms of resistance include secondary mutations in SMO, downstream amplification of GLI2 and also aberrant activation of PI3K/AKT/mTOR signaling (Buonamici et al., 2010; Dijkgraaf et al., 2011; Rudin et al., 2009; Yauch et al., 2009).

\subsubsection{Comparison of cooperative antitumor effects of SMO antagonists in combination with PI3K/AKT/mTOR inhibitors in RD cells}

The data in this thesis demonstrate that HhA was the only SMO antagonists with an inhibitory effect on PI3K/AKT/mTOR signaling. Therefore, HhA was the best candidate for combination with PI3K/AKT/mTOR inhibitors. Nevertheless, in several initial experiments the anticancer effects of GDC-0449, LDE225, HhA and cyclopamine in combination with the latter drugs were compared in RD cells. For this purpose apoptosis was measured by AnnexinV labeling of the 
cells and the impact on phosphorylation of AKT, S6 and AMPK and on other proteins was examined by western blot.

Concerning AnnexinV positivity, RD cells were more sensitive to $3 \mu \mathrm{M}$ PI103 and $10 \mu \mathrm{M}$ GDC-0941 than to treatment with the SMO inhibitors (except for $30 \mu \mathrm{M}$ LDE225 and HhA; Figure 17). This again indicated a strong dependency of RMS cells on PI3K/AKT/mTOR signaling. The observation that MK-2206 or both mTOR inhibitors alone induced marginal or even no proapoptotic effects was astonishing. Lack of proapoptotic effects upon treatment of RMS cell lines with everolimus has also been shown by other groups, even at a concentration of $1 \mu \mathrm{M}$ (Preuss et al., 2013). It is possible that for efficient apoptosis induction, the cells require a combined inhibition of pAKT and mTOR (indicated by decreased pS6 levels), which was observed for PI103 and GDC-0941, but not for MK-2206, everolimus or rapamycin (see e.g. Figure 18). However, this cannot explain the strong apoptosis induction resulting from the combination of $10 \mu \mathrm{M}$ HhA plus MK-2206 (Figure $17 \mathrm{C}$ ) because this treatment did not lead to a decrease in pS6 (Figure 20). In this case it is rather possible that HhA or MK-2206 modulate additional factors that are responsible for this effect.

The specific PI3K inhibitor GDC-0941 blocked phosphorylation of AKT as well as of S6 in all RMS cell lines (Figure 27 - Figure 30). Because PI3K activates PDK1 (Cantley, 2002), which can also control $\mathrm{mTOR}_{\mathrm{C} 1}$ activity (Finlay et al., 2012), inhibition of PI3K/PDK1 upon GDC-0941 could possibly reduce pS6 levels in addition to pAKT. Nevertheless since the catalytic subunit of PI3K and mTOR are structurally similar, GDC-0941 probably exerts inhibitory effects on both proteins.

HhA was the most efficient compound when combined with PI3K/AKT/mTOR inhibitors. Combination effects were not that convincing when GDC-0449, LDE225 or cyclopamine were used. This is similar to a report by Graab et al., who also demonstrated that the combination of GDC-0449 plus PI103 failed to induce cooperative effects on apoptosis in RMS cell lines (Graab et al., 2015). In contrast to the other SMO antagonists, HhA induced cooperative effects, e.g. it reversed the increase of pAKT levels caused by mTOR inhibitors and reduced AMPK phosphorylation when combined with PI103, GDC-0941 or MK-2206 (Figure 20). Moreover, HhA showed cooperative proapoptotic effects with several of the used PI3K/AKT/mTOR inhibitors as determined by AnnexinV positive cells (Figure $17 \mathrm{C}$ ).

Indeed HhA increased number of AnnexinV positive RD cells (Figure $17 \mathrm{C}$ ) but without stimulating caspase 3 activity (Figure 20). This indicated a caspase 3 independent proapoptotic mechanism. This was similar for PI103 and GDC-0941 in combination with $10 \mu \mathrm{M}$ GDC-0449 and HhA that also increased the number of AnnexinV positive RD cells (Figure $17 \mathrm{~A}, \mathrm{C}$ ), 


\section{DISCUSSION}

whereas caspase 3 activity was rather repressed by these combinations (Figure 18, Figure 20). Similar results were observed in RMS-13 cells for HhA plus PI103 or GDC-0941 (compare Figure 26 B with Figure 29). These findings suggest that besides caspase 3 activation other mechanisms could contribute to apoptosis induction by the respective drugs and their combinations. One possibility is that the cells undergo apoptosis due to activation of other effector caspases such as caspase 6 or 7. Moreover, several other groups demonstrated that also caspase 3 independent pathways could contribute to apoptosis (Alvarez et al., 2011; Bai et al., 2015). Furthermore complex interactions between apoptosis and autophagy are known, in which both pathways either cooperate, assist or antagonize each other (reviewed in (Nikoletopoulou et al., 2013)). Therefore, it is possible that autophagy may have contributed to caspase 3 independent apoptosis. This is supported by the observation that apoptosis was accompanied by autophagy in nearly all experimental settings.

Together the data showed that HhA was the most promising compound for combination with $\mathrm{PI} 3 \mathrm{~K} / \mathrm{AKT} / \mathrm{mTOR}$ inhibitors. Moreover and as already described, single drug treatment with HhA resulted in antiproliferative and proapoptotic effects, inhibited pAKT and induced LC3-II. Therefore, the impact of HhA plus PI3K/AKT/mTOR inhibitors was studied in more detail and in additional human RMS cell lines.

\subsubsection{Detailed analyses of cooperative antitumoral effects of HhA plus PI3K/AKT/mTOR inhibitors}

As already described in section 7.1.1, nearly all PI3K/AKT/mTOR inhibitors efficiently blocked HH signaling activity in almost all RMS cell lines (Figure 21). Similar results were obtained by other groups (Graab et al., 2015; Kaylani et al., 2013). Interestingly, the strongest GLII inhibition was revealed with GDC-0941, which had more potency than the SMO inhibitor HhA (Figure 21). This may indicate that PI3K (that is targeted by GDC-0941) has an enormous influence on GLI transcription. The phosphorylation of PIP2 to PIP3, catalyzed by PI3K, leads to activation of various kinases including PDK1 (Cantley, 2002). PDK1 activates AKT, but it has also other targets e.g. PKC $\delta$ (Le Good et al., 1998), which is involved in GLI activation (Lauth et al., 2007). Therefore it is possible that inhibition of PI3K and consequently PDK1/PKC $\delta$ (together with AKT inhibition) is responsible for the strong repression of GLI transcription. Since RMS-13 cells harbor a GLII amplification (Roberts et al., 1989), but GLI1 expression was still decreased by $\mathrm{PI} 3 \mathrm{~K} / \mathrm{AKT} / \mathrm{mTOR}$ inhibitors, it is also possible that components of the 
PI3K/AKT/mTOR pathway stabilize GLI transcription factors and maintain their transcriptional activity as already described by others (Riobo et al., 2006; Wang et al., 2012).

Interestingly, when $30 \mu \mathrm{M}$ HhA was combined with MK-2206 in RD cells GLII expression was slightly increased compared to the single drug treatments (Figure $21 \mathrm{~A}$ ). This went along with an enormous apoptosis induction (Figure $17 \mathrm{C}$, Figure 27) probably caused by complete blocking of AKT. Since this combination inhibited the activity of AKT together with that of mTOR (Figure 27), it is possible that PI3K was strongly activated due to loss of negative feedback by the S6K/IRS/PI3K axis (see section 2.6). Therefore, overactivated PI3K via PDK1/PKC $\delta$ may have induced GLII transcription despite inactivation of AKT.

Nevertheless, combination treatment of RMS cells with HhA plus PI3K/AKT/mTOR inhibitors rarely showed cooperative effects on GLII suppression. The lack of cooperative effects on GLII transcription was not very surprising, because HhA was in most cell lines not able to reduce GLII expression (Figure 21). These data again support the assumption that GLII expression in the analyzed RMS cell lines is regulated in a noncanonical manner by the PI3K/AKT/mTOR pathway.

The analysis of IGF2 expression revealed no cooperative effects of the combinations in all RMS cell lines (Figure 22). In contrast to mTOR inhibitors, which rather enhanced IGF2 transcription, PI103, GDC-0941 and MK-2206 reduced IGF2 expression in most settings. One possibility for these results is that PI103, GDC-0941 and MK-2206 inhibited AKT (Figure 27, Figure 29, Figure 30). Since AKT represses p53 via MDM2 (see section 2.6), inhibition of AKT may led to induction of p53 action and finally to repression of IGF2 transcription, because IGF2 expression is suppressed by p53 in multiple cell lines including RMS cell lines (Zhang et al., 1996; Zhang et al., 1998a). In contrast to that, mTOR inhibitors lead to an overactivation of AKT in RD and Rh41 cells (Figure 27, Figure 30) which in turn may have blocked p53 and consequently induced IGF2 transcription. Because IGF2 can act as an autocrine growth factor in RMS cells (El-Badry et al., 1990; Minniti et al., 1994) and induces phosphorylation of AKT (Hartmann et al., 2005), this could have additionally strengthened the activation of AKT upon mTOR inhibition.

Expression of the muscle differentiation markers $M Y O D$ and $M Y H 1$ was in most cases unaffected upon single drug treatment with MK-2206 and mTOR inhibitors, whereas PI103 and GDC-0941 rather decreased their expression (Figure 23, Figure 24). However, these effects were cell line dependent. As already described in chapter 7.1.2, the expression pattern of the muscle differentiation markers may be associated with IGF2 expression, e.g. RD and RMS-13 cells 


\section{DISCUSSION}

showed co-regulation of $M Y O D$ and $I G F 2$ levels (compare Figure $23 \mathrm{~A}, \mathrm{~B}$ with Figure $22 \mathrm{~A}, \mathrm{~B}$ ). A similar correlation has been described by Wilson and colleagues who showed that Igf2 inhibition resulted in decreased $M y o D$ expression and that vice versa MyoD increased Igf2 transcription (Wilson et al., 2003; Wilson and Rotwein, 2006).

Similar to GLII, the data on IGF2, MYOD and MYH1 expression suggest that combination treatment of HhA plus PI3K/AKT/mTOR inhibitors did not strengthen the effects of the single drug treatments in most settings. These findings indicate that concerning the latter factors, combination of HhA plus inhibitors of the PI3K/AKT/mTOR pathway do not represent an improvement over single drug treatment.

In general, the analysis of the combination effects on the PI3K/AKT/mTOR pathway, activation of AMPK and on LC3-II levels revealed that HhA plus PI3K/AKT/mTOR inhibitors induced variable outcomes among the analyzed RMS cell lines, which were more heterogeneous in ARMS than in ERMS.

For instance the combinations of HhA plus PI3K/AKT/mTOR inhibitors reduced pAMPK levels in RD cells, but mostly induced them in RMS-13 and did not modulate them in RUCH-2 cells. Although these results are preliminary, it is tempting to speculate that inhibition of pAMPK upon combination of HhA with PI103, GDC-0941 or MK-2206 (as an example see RD cells, Figure 27) would be beneficial for RMS therapy, because the pAMPK level is positively correlated with the induction of a Warburg like effect. The Warburg effect describes the metabolic shift from mitochondrial oxidative phosphorylation towards aerobic glycolysis, which is observed in cancer cells. This leads to several proliferative and survival advantages of the cancer cells, e.g. a decreased dependence on oxygen (reviewed in (Hsu and Sabatini, 2008)). Hence, a reduction of pAMPK levels (indicating the repression of Warburg like metabolism) by the mentioned drug combinations might decrease the viability of RMS cells. Furthermore, the physiological outcome of AMPK mediated catabolism may be responsible for side effects upon therapy with SMO inhibitors i.e. muscle cramping and weight loss (Teperino et al., 2012). Therefore, an inhibition of AMPK by the mentioned combination treatment could avoid these side effects.

LC3-II as a marker for autophagy was examined. Other groups have demonstrated that active $\mathrm{HH}$ signaling inhibits autophagy and vice versa inactivation of $\mathrm{HH}$ signaling using the GLI inhibitor GANT61 induces autophagy (Jimenez-Sanchez et al., 2012; Wang et al., 2013; Xu et al., 2014). Indeed, PI3K/AKT/mTOR inhibitors efficiently inhibited GLII transcription (e.g. PI103 and GDC-0941 in all cells, Figure 21) and thus HH signaling, which could explain the 
increased LC3-II levels observed by most of the PI3K/AKT/mTOR inhibitors (Figure 27 - Figure 30). However these findings were again strongly dependent on the cell line and a clear correlation between GLII inhibition and LC3-II induction was not always seen, e.g. PI103 or GDC-0941 decreased GLI1 expression in Rh41 cells, but LC3-II levels were not modulated (compare Figure 21 D with Figure 30).

Nevertheless, inhibition of PI3K/AKT/mTOR signaling is well known to result in autophagy because this pathway (and mainly mTOR) regulates autophagy (reviewed in (Chen and Karantza, 2011). In our settings everolimus and rapamycin did not enhance LC3-II expression as strong as observed for PI103, GDC-0941 or MK-2206 (e.g. in RD and RUCH-2 cells; Figure 18, Figure 28) or they did not induce LC3-II expression at all (e.g. in Rh41, Figure 30). When everolimus and rapamycin were combined with HhA, LC3-II expression was increased up to HhA induced levels in most of the cell lines. Cooperative effects on LC3-II were e.g. observed in RUCH-2 cells, when HhA was combined with PI103, GDC-0941 or mTOR inhibitors (Figure 28).

Because the role of autophagy in cancer is controversial and complex and because it could function as a tumor suppressor or act as a protector of cancer cell survival (reviewed in (White and DiPaola, 2009)), it was tried to correlate the increase in LC3-II with antiproliferative or proapoptotic effects of $\mathrm{HhA}$ and/or PI3K/AKT/mTOR inhibitors. However, the increase in LC3-II was not unequivocally positively or negatively related to the anticancer effects, probably due to drug and combination specific effects and because of the cellular context. Therefore it cannot be judged whether the observed increase of LC3-II levels are beneficial or disadvantageous for RMS treatment with the respective drugs.

In contrast to HhA, PI103 and GDC-0941, the mTOR inhibitors everolimus and rapamycin increased the proliferation rate of some cells (Figure 25) and did not modulate apoptosis (they neither increased the number of AnnexinV positive cells nor activated caspase 3; Figure 26, Figure 27 - Figure 30). As already mentioned, this can be explained by induction of IGF2 expression (Figure 22) and by activation of AKT (Figure 27, Figure 30) in some settings. Overactivation of AKT due to treatment with mTOR inhibitors has been frequently demonstrated by others in various cell lines including RMS (O'Reilly et al., 2006; Tamburini et al., 2008; Wan et al., 2007). The reason for the increased AKT activation is that inhibition of $\mathrm{mTOR}_{\mathrm{C} 1}$ releases the negative feedback by the S6K/IRS/PI3K axis, which in turn can paradoxically overactivate AKT. These effects might limit their application for RMS therapy. Indeed, rapamycin treatment of RMS xenografts generated from six different RMS cell lines showed only in two cases valuable growth inhibition and the overall response rate in a panel of sarcoma cell lines was 


\section{DISCUSSION}

relatively low (Houghton et al., 2008). Although the combination with HhA mostly abrogated these unwanted effects, the use of everolimus and rapamycin in RMS therapy should be carefully reconsidered.

Growth inhibition and apoptosis induction are the most important parameters of an efficient antitumor treatment. The data here show that single drug treatment with HhA, PI103 and GDC-0941 inhibited proliferation (Figure 25) and induced proapoptotic effects (Figure 26) in all RMS cell lines. Importantly, cooperative antiproliferative and proapoptotic effects were detected for the combination of HhA plus PI103, HhA plus GDC-0941 and HhA plus MK-2206 in most of the cell lines (Figure 25, Figure 26). Therefore, the cell culture experiments suggested that these combinations might be promising treatment options for both, ERMS and ARMS, and could be further employed for RMS therapy.

Since GDC-0941 is used in clinical trials (in contrast to PI103) and showed the most convincing effects on growth inhibition in all RMS cell lines (in contrast to MK-2206, which induced proliferation of some cell lines; Figure 25 A, C), HhA plus GDC-0941 were applied to RMS bearing Ptch $^{+/}$mice. As single substances both HhA and GDC-0941 were able to efficiently stop the growth of the tumors, whereas GDC-0941 showed more potency than HhA (Figure 31). These findings again suggest that besides Hh signaling pathway, the PI3K/Akt/mTor cascade plays an important role in RMS. In addition, this also implies that the PI3K/Akt/mTor pathway is also crucial for those RMS, in which Hh signaling is activated due to Ptch mutations, as it is the case for RMS of $\mathrm{Ptch}^{+-}$mice. In these mice Hh signaling is most likely activated in a SMO dependent (i.e. canonical) manner due to the Ptch mutation. However, the tumors of these animals also show activation of the PI3K/Akt/mTor pathway (Kappler et al., 2003) and tumor growth is decreased upon GDC-0941 treatment (Figure 31). Thus, RMS of Ptch ${ }^{+/}$mice resemble human RMS cell lines and represent a suitable model for the preclinical evaluation of both $\mathrm{HH}$ and PI3K/AKT/mTOR inhibitors.

Similar to the in vitro data, the combination of HhA plus GDC-0941 induced a cooperative growth inhibitory effect on murine RMS (compared to the control and to single treatment with HhA). This is comparable to studies that combined LDE225 with the dual PI3K/mTOR inhibitor NVP-BEZ235 in murine Ptch mutant MB (Buonamici et al., 2010) or with the PI3K inhibitor NVP-BKM120 in PTEN deficient glioblastomas (Filbin et al., 2013). Interestingly, the cooperative effect on RMS growth inhibition was not reflected by a further decrease in Ki67 positive cells compared to either drug alone (Figure $32 \mathrm{~B}$ ). Accordingly, cooperative RMS growth inhibition by HhA plus GDC-0941 is most likely not exclusively related to inhibition of 
proliferation and might be additionally associated with other antitumoral effects (e.g. induction of apoptosis or autophagic cell death).

Cyclopamine enhanced RMS tumor growth in vivo (Ecke et al., 2008) and did not decrease the amount of Ki67 positive cells of the respective tumors (Figure $32 \mathrm{C}$ ). This confirmed the in vitro effects of cyclopamine in this thesis in which cyclopamine did not decreased proliferation or rather induced it (Figure 10). Moreover, these contrary outcomes of HhA and cyclopamine on RMS growth in $\mathrm{Ptch}^{+/}$mice, confirmed again the heterogeneous effects of SMO antagonists on RMS found in this thesis.

In conclusion, the data strongly suggest that combined inhibition of the $\mathrm{HH}$ and the $\mathrm{PI} 3 \mathrm{~K} / \mathrm{AKT} / \mathrm{mTOR}$ signaling pathways could be a treatment strategy to improve therapy of RMS. Single drug treatment with some PI3K/AKT/mTOR inhibitors alone already showed antitumoral effects in human RMS cell lines (e.g. inhibition of proliferation and induction of apoptosis), which was not seen with everolimus and rapamycin. Combination of the SMO antagonists HhA with PI103, GDC-0941 or MK-2206 enhanced anticancer effects, which demonstrates a more effective treatment option than the corresponding single drug treatments. Particularly the combination of HhA plus the pure PI3K inhibitor GDC-0941 intensifies numerous antitumoral effects in the analyzed human RMS cell lines and hence represented the most promising drug combination. Indeed, cooperative growth inhibitory effects of this combination have been observed in both, human RMS cell lines and in RMS of $\mathrm{Ptch}^{+/}$mice. Unfortunately, the experiments performed in the frame of this thesis cannot explain the underlying mechanisms. However, the response of RMS cell lines highly depends on the used SMO antagonist and is also influenced by the used PI3K/AKT/mTOR inhibitor and thus by the respective combinations. Due to this heterogeneous response of RMS, it will be necessary to conduct thorough pretesting of the substances and combinations before applying these drugs to RMS patients. This could be done either in patient derived short term RMS cultures or in patient derived xenograft mouse models.

\subsection{Outlook}

In the future, preliminary findings have to be validated and therefore repeated. This includes the analysis of the effects upon SAG treatment on AKT and GLII expression. Moreover, the western blot analyses of the comparison of the SMO antagonists plus PI3K/AKT/mTOR inhibitors in RD cells should be repeated as well as the western blots of HhA plus PI3K/AKT/mTOR inhibitors in 


\section{DISCUSSION}

RUCH-2, RMS-13 and Rh41 cells. Finally, the gene expression studies of HhA plus $\mathrm{PI} 3 \mathrm{~K} / \mathrm{AKT} / \mathrm{mTOR}$ inhibitors in RUCH-2 and Rh41 cells have to be replicated.

In order to unravel the molecular mechanisms contributing to the observed effects of SMO antagonists and/or PI3K/AKT/mTOR inhibitors, the following experiments should be performed.

To exclude that the effects of the SMO antagonists are off-target effects, loss-of-function experiments on SMO should be performed e.g. by SMO knockdown.

In order to verify that the $\mathrm{HH}$ pathway is indeed activated in a noncanonical manner by PI3K/AKT/mTOR signaling, e.g. PI3K or AKT could be activated by transfection of the RMS cells with constitutively active AKT or PI3K isoforms. Conversely, the respective proteins could be blocked either by overexpression of respective dominant negative proteins or by knockdown. In order to prove that the effects induced by SMO inhibitors are indeed independent of canonical $\mathrm{HH}$ signaling, all three GLI transcription factors should be silenced by mean of siRNA technology.

Moreover, it will be interesting to compare the gene expression profiles within one RMS cell line, which has been treated with different SMO inhibitors with various outcomes. This could give some information about the individual properties of the specific drugs. Vice versa it would be interesting to compare the gene expression profiles between different cell lines that were treated with the same SMO inhibitor with different responsiveness.

Another interesting observation is the HhA mediated increase in AnnexinV positive cells, accompanied by either absent or reduced caspase 3 activity, which was also seen upon some drug combinations. In order to show that apoptosis is indeed caspase independent, caspase 3 or pan caspase inhibitors could be applied.

While autophagy was often found to be induced upon specific drug treatments, it was not clearly defined whether this would be beneficial or unfavorable. Applying autophagy inhibitors together with the drugs would distinguish between a tumor suppressor and a tumor survival function of autophagy during the treatment with the drug and define their role in RMS therapy.

It would also be important to unravel the mechanisms underlying the tumor response after the in vivo treatment with HhA and/or GDC-0941. The same parameters as in the human RMS cell lines could be investigated (i.e. analysis of pAkt, pS6, Lc3-II, pAmpk, Igf2 via immunohistochemistry and western blot, as well as Glil expression and muscle differentiation markers via qRT-PCR). 
Finally, it also would be worth analyzing the in vivo response of RMS towards GDC-0449 and LDE225 alone or in combination with a PI3K and/or AKT inhibitor. Although these drugs did not unequivocally exert antigrowth effects in cell culture, they might exert antitumoral effects in vivo. 


\section{REFERENCES}

Aberger, F., Kern, D., Greil, R., Hartmann, T.N., 2012. Canonical and noncanonical Hedgehog/GLI signaling in hematological malignancies. Vitamins and hormones 88, 2554.

Agarwal, E., Chaudhuri, A., Leiphrakpam, P.D., Haferbier, K.L., Brattain, M.G., Chowdhury, S., 2014. Akt inhibitor MK-2206 promotes anti-tumor activity and cell death by modulation of AIF and Ezrin in colorectal cancer. BMC cancer 14, 145.

Alvarez, S., Blanco, A., Fresno, M., Munoz-Fernandez, M.A., 2011. TNF-alpha contributes to caspase-3 independent apoptosis in neuroblastoma cells: role of NFAT. PloS one 6, e16100.

Amakye, D., Jagani, Z., Dorsch, M., 2013. Unraveling the therapeutic potential of the Hedgehog pathway in cancer. Nature medicine 19, 1410-1422.

Bai, X., Kinney, W.H., Su, W.L., Bai, A., Ovrutsky, A.R., Honda, J.R., Netea, M.G., HenaoTamayo, M., Ordway, D.J., Dinarello, C.A., Chan, E.D., 2015. Caspase-3-independent apoptotic pathways contribute to interleukin-32gamma-mediated control of Mycobacterium tuberculosis infection in THP-1 cells. BMC microbiology 15, 39.

Barnes, E.A., Kong, M., Ollendorff, V., Donoghue, D.J., 2001. Patched1 interacts with cyclin B1 to regulate cell cycle progression. The EMBO journal 20, 2214-2223.

Beachy, P.A., Karhadkar, S.S., Berman, D.M., 2004. Tissue repair and stem cell renewal in carcinogenesis. Nature 432, 324-331.

Belgacem, Y.H., Borodinsky, L.N., 2011. Sonic hedgehog signaling is decoded by calcium spike activity in the developing spinal cord. Proceedings of the National Academy of Sciences of the United States of America 108, 4482-4487.

Berman, D.M., Karhadkar, S.S., Hallahan, A.R., Pritchard, J.I., Eberhart, C.G., Watkins, D.N., Chen, J.K., Cooper, M.K., Taipale, J., Olson, J.M., Beachy, P.A., 2002. Medulloblastoma growth inhibition by hedgehog pathway blockade. Science 297, 1559-1561.

Berman, D.M., Karhadkar, S.S., Maitra, A., Montes De Oca, R., Gerstenblith, M.R., Briggs, K., Parker, A.R., Shimada, Y., Eshleman, J.R., Watkins, D.N., Beachy, P.A., 2003. Widespread requirement for Hedgehog ligand stimulation in growth of digestive tract tumours. Nature 425, 846-851.

Bigelow, R.L., Chari, N.S., Unden, A.B., Spurgers, K.B., Lee, S., Roop, D.R., Toftgard, R., McDonnell, T.J., 2004. Transcriptional regulation of bcl-2 mediated by the sonic hedgehog signaling pathway through gli-1. The Journal of biological chemistry 279, 1197-1205.

Binns, W., James, L.F., Keeler, R.F., Balls, L.D., 1968. Effects of teratogenic agents in range plants. Cancer research 28, 2323-2326.

Bren-Mattison, Y., Olwin, B.B., 2002. Sonic hedgehog inhibits the terminal differentiation of limb myoblasts committed to the slow muscle lineage. Developmental biology 242, 130148.

Breneman, J.C., Lyden, E., Pappo, A.S., Link, M.P., Anderson, J.R., Parham, D.M., Qualman, S.J., Wharam, M.D., Donaldson, S.S., Maurer, H.M., Meyer, W.H., Baker, K.S., Paidas, C.N., Crist, W.M., 2003. Prognostic factors and clinical outcomes in children and adolescents with metastatic rhabdomyosarcoma-a report from the Intergroup Rhabdomyosarcoma Study IV. Journal of clinical oncology : official journal of the American Society of Clinical Oncology 21, 78-84. 
Brennan, D., Chen, X., Cheng, L., Mahoney, M., Riobo, N.A., 2012. Noncanonical Hedgehog signaling. Vitamins and hormones 88, 55-72.

Bridge, J.A., Liu, J., Weibolt, V., Baker, K.S., Perry, D., Kruger, R., Qualman, S., Barr, F., Sorensen, P., Triche, T., Suijkerbuijk, R., 2000. Novel genomic imbalances in embryonal rhabdomyosarcoma revealed by comparative genomic hybridization and fluorescence in situ hybridization: an intergroup rhabdomyosarcoma study. Genes Chromosomes Cancer 27, 337-344.

Buonamici, S., Williams, J., Morrissey, M., Wang, A., Guo, R., Vattay, A., Hsiao, K., Yuan, J., Green, J., Ospina, B., Yu, Q., Ostrom, L., Fordjour, P., Anderson, D.L., Monahan, J.E., Kelleher, J.F., Peukert, S., Pan, S., Wu, X., Maira, S.M., Garcia-Echeverria, C., Briggs, K.J., Watkins, D.N., Yao, Y.M., Lengauer, C., Warmuth, M., Sellers, W.R., Dorsch, M., 2010. Interfering with resistance to smoothened antagonists by inhibition of the PI3K pathway in medulloblastoma. Sci Transl Med 2, 51ra70.

Calzada-Wack, J., Schnitzbauer, U., Walch, A., Wurster, K.H., Kappler, R., Nathrath, M., Hahn, H., 2002. Analysis of the PTCH coding region in human rhabdomyosarcoma. Hum Mutat 20, 233-234.

Cantley, L.C., 2002. The phosphoinositide 3-kinase pathway. Science 296, 1655-1657.

Carracedo, A., Pandolfi, P.P., 2008. The PTEN-PI3K pathway: of feedbacks and cross-talks. Oncogene 27, 5527-5541.

Chen, J.K., Taipale, J., Cooper, M.K., Beachy, P.A., 2002a. Inhibition of Hedgehog signaling by direct binding of cyclopamine to Smoothened. Genes \& development 16, 2743-2748.

Chen, J.K., Taipale, J., Young, K.E., Maiti, T., Beachy, P.A., 2002b. Small molecule modulation of Smoothened activity. Proceedings of the National Academy of Sciences of the United States of America 99, 14071-14076.

Chen, N., Karantza, V., 2011. Autophagy as a therapeutic target in cancer. Cancer biology \& therapy $11,157-168$.

Chinchilla, P., Xiao, L., Kazanietz, M.G., Riobo, N.A., 2010. Hedgehog proteins activate proangiogenic responses in endothelial cells through non-canonical signaling pathways. Cell Cycle 9, 570-579.

Clement, V., Sanchez, P., de Tribolet, N., Radovanovic, I., Ruiz i Altaba, A., 2007. HEDGEHOG-GLI1 signaling regulates human glioma growth, cancer stem cell selfrenewal, and tumorigenicity. Curr Biol 17, 165-172.

Cohen, P., Frame, S., 2001. The renaissance of GSK3. Nature reviews. Molecular cell biology 2, 769-776.

Cooper, M.K., Porter, J.A., Young, K.E., Beachy, P.A., 1998. Teratogen-mediated inhibition of target tissue response to Shh signaling. Science 280, 1603-1607.

Corcoran, R.B., Bachar Raveh, T., Barakat, M.T., Lee, E.Y., Scott, M.P., 2008. Insulin-like growth factor 2 is required for progression to advanced medulloblastoma in patched1 heterozygous mice. Cancer research 68, 8788-8795.

Courtney, K.D., Corcoran, R.B., Engelman, J.A., 2010. The PI3K pathway as drug target in human cancer. Journal of clinical oncology : official journal of the American Society of Clinical Oncology 28, 1075-1083.

D'Amato, C., Rosa, R., Marciano, R., D'Amato, V., Formisano, L., Nappi, L., Raimondo, L., Di Mauro, C., Servetto, A., Fulciniti, F., Cipolletta, A., Bianco, C., Ciardiello, F., Veneziani, B.M., De Placido, S., Bianco, R., 2014. Inhibition of Hedgehog signalling by NVPLDE225 (Erismodegib) interferes with growth and invasion of human renal cell carcinoma cells. British journal of cancer 111, 1168-1179.

Dagher, R., Helman, L., 1999. Rhabdomyosarcoma: an overview. The oncologist 4, 34-44. 
Das, S., Samant, R.S., Shevde, L.A., 2013. Nonclassical activation of Hedgehog signaling enhances multidrug resistance and makes cancer cells refractory to Smoothened-targeting Hedgehog inhibition. The Journal of biological chemistry 288, 11824-11833.

De Giovanni, C., Landuzzi, L., Nicoletti, G., Lollini, P.L., Nanni, P., 2009. Molecular and cellular biology of rhabdomyosarcoma. Future Oncol 5, 1449-1475.

de Souza, R.R., Oliveira, I.D., del Giudice Paniago, M., Yaoita, F.H., Caran, E.M., Macedo, C.R., Petrilli, A.S., Abib Sde, C., de Seixas Alves, M.T., de Toledo, S.R., 2014. Investigation of IGF2, Hedgehog and fusion gene expression profiles in pediatric sarcomas. Growth hormone \& IGF research : official journal of the Growth Hormone Research Society and the International IGF Research Society 24, 130-136.

Denef, N., Neubuser, D., Perez, L., Cohen, S.M., 2000. Hedgehog induces opposite changes in turnover and subcellular localization of patched and smoothened. Cell 102, 521-531.

Dickerman, J.D., 2007. The late effects of childhood cancer therapy. Pediatrics 119, 554-568.

Dijkgraaf, G.J., Alicke, B., Weinmann, L., Januario, T., West, K., Modrusan, Z., Burdick, D., Goldsmith, R., Robarge, K., Sutherlin, D., Scales, S.J., Gould, S.E., Yauch, R.L., de Sauvage, F.J., 2011. Small molecule inhibition of GDC-0449 refractory smoothened mutants and downstream mechanisms of drug resistance. Cancer research 71, 435-444.

Duman-Scheel, M., Weng, L., Xin, S., Du, W., 2002. Hedgehog regulates cell growth and proliferation by inducing Cyclin D and Cyclin E. Nature 417, 299-304.

Ecke, I., Rosenberger, A., Obenauer, S., Dullin, C., Aberger, F., Kimmina, S., Schweyer, S., Hahn, H., 2008. Cyclopamine treatment of full-blown Hh/Ptch-associated RMS partially inhibits Hh/Ptch signaling, but not tumor growth. Molecular carcinogenesis 47, 361-372.

Egas-Bejar, D., Huh, W.W., 2014. Rhabdomyosarcoma in adolescent and young adult patients: current perspectives. Adolescent health, medicine and therapeutics 5, 115-125.

El-Badry, O.M., Minniti, C., Kohn, E.C., Houghton, P.J., Daughaday, W.H., Helman, L.J., 1990. Insulin-like growth factor II acts as an autocrine growth and motility factor in human rhabdomyosarcoma tumors. Cell Growth Differ 1, 325-331.

Fan, Q.W., Knight, Z.A., Goldenberg, D.D., Yu, W., Mostov, K.E., Stokoe, D., Shokat, K.M., Weiss, W.A., 2006. A dual PI3 kinase/mTOR inhibitor reveals emergent efficacy in glioma. Cancer cell 9, 341-349.

Fasolo, A., Sessa, C., 2012. Targeting mTOR pathways in human malignancies. Current pharmaceutical design 18, 2766-2777.

Feldmann, G., Dhara, S., Fendrich, V., Bedja, D., Beaty, R., Mullendore, M., Karikari, C., Alvarez, H., Iacobuzio-Donahue, C., Jimeno, A., Gabrielson, K.L., Matsui, W., Maitra, A., 2007. Blockade of hedgehog signaling inhibits pancreatic cancer invasion and metastases: a new paradigm for combination therapy in solid cancers. Cancer research 67, 2187-2196.

Filbin, M.G., Dabral, S.K., Pazyra-Murphy, M.F., Ramkissoon, S., Kung, A.L., Pak, E., Chung, J., Theisen, M.A., Sun, Y., Franchetti, Y., Shulman, D.S., Redjal, N., Tabak, B., Beroukhim, R., Wang, Q., Zhao, J., Dorsch, M., Buonamici, S., Ligon, K.L., Kelleher, J.F., Segal, R.A., 2013. Coordinate activation of Shh and PI3K signaling in PTENdeficient glioblastoma: new therapeutic opportunities. Nature medicine 19, 1518-1523.

Finlay, D.K., Rosenzweig, E., Sinclair, L.V., Feijoo-Carnero, C., Hukelmann, J.L., Rolf, J., Panteleyev, A.A., Okkenhaug, K., Cantrell, D.A., 2012. PDK1 regulation of mTOR and hypoxia-inducible factor 1 integrate metabolism and migration of CD8+ T cells. The Journal of experimental medicine 209, 2441-2453.

Fu, J.R., Liu, W.L., Zhou, J.F., Sun, H.Y., Xu, H.Z., Luo, L., Zhang, H., Zhou, Y.F., 2006. Sonic hedgehog protein promotes bone marrow-derived endothelial progenitor cell proliferation, migration and VEGF production via PI 3-kinase/Akt signaling pathways. Acta Pharmacol Sin 27, 685-693. 
Ganti, R., Skapek, S.X., Zhang, J., Fuller, C.E., Wu, J., Billups, C.A., Breitfeld, P.P., Dalton, J.D., Meyer, W.H., Khoury, J.D., 2006. Expression and genomic status of EGFR and ErbB-2 in alveolar and embryonal rhabdomyosarcoma. Modern pathology : an official journal of the United States and Canadian Academy of Pathology, Inc 19, 1213-1220.

Gerber, A.N., Wilson, C.W., Li, Y.J., Chuang, P.T., 2006. The hedgehog regulated oncogenes Gli1 and Gli2 block myoblast differentiation by inhibiting MyoD-mediated transcriptional activation. Oncogene.

Goodrich, L.V., Milenkovic, L., Higgins, K.M., Scott, M.P., 1997. Altered neural cell fates and medulloblastoma in mouse patched mutants. Science 277, 1109-1113.

Gorlin, R.J., 1987. Nevoid basal-cell carcinoma syndrome. Medicine (Baltimore) 66, 98-113.

Gorojankina, T., Hoch, L., Faure, H., Roudaut, H., Traiffort, E., Schoenfelder, A., Girard, N., Mann, A., Manetti, F., Solinas, A., Petricci, E., Taddei, M., Ruat, M., 2013. Discovery, molecular and pharmacological characterization of GSA-10, a novel small-molecule positive modulator of Smoothened. Molecular pharmacology 83, 1020-1029.

Graab, U., Hahn, H., Fulda, S., 2015. Identification of a novel synthetic lethality of combined inhibition of hedgehog and PI3K signaling in rhabdomyosarcoma. Oncotarget.

Guertin, D.A., Sabatini, D.M., 2009. The pharmacology of mTOR inhibition. Science signaling 2 , pe24.

Hahn, H., Wicking, C., Zaphiropoulous, P.G., Gailani, M.R., Shanley, S., Chidambaram, A., Vorechovsky, I., Holmberg, E., Unden, A.B., Gillies, S., Negus, K., Smyth, I., Pressman, C., Leffell, D.J., Gerrard, B., Goldstein, A.M., Dean, M., Toftgard, R., Chenevix-Trench, G., Wainwright, B., Bale, A.E., 1996. Mutations of the human homolog of Drosophila patched in the nevoid basal cell carcinoma syndrome. Cell 85, 841-851.

Hahn, H., Wojnowski, L., Miller, G., Zimmer, A., 1999. The patched signaling pathway in tumorigenesis and development: lessons from animal models [In Process Citation]. J Mol Med 77, 459-468.

Hahn, H., Wojnowski, L., Specht, K., Kappler, R., Calzada-Wack, J., Potter, D., Zimmer, A., Muller, U., Samson, E., Quintanilla-Martinez, L., 2000. Patched target Igf2 is indispensable for the formation of medulloblastoma and rhabdomyosarcoma. The Journal of biological chemistry $275,28341-28344$.

Hahn, H., Wojnowski, L., Zimmer, A.M., Hall, J., Miller, G., Zimmer, A., 1998. Rhabdomyosarcomas and radiation hypersensitivity in a mouse model of Gorlin syndrome. Nature Med 4, 619-622.

Hartmann, W., Koch, A., Brune, H., Waha, A., Schuller, U., Dani, I., Denkhaus, D., Langmann, W., Bode, U., Wiestler, O.D., Schilling, K., Pietsch, T., 2005. Insulin-like growth factor II is involved in the proliferation control of medulloblastoma and its cerebellar precursor cells. Am J Pathol 166, 1153-1162.

Hawkins, D.S., Gupta, A.A., Rudzinski, E.R., 2014. What is new in the biology and treatment of pediatric rhabdomyosarcoma? Current opinion in pediatrics $26,50-56$.

Hawkins, D.S., Spunt, S.L., Skapek, S.X., 2013. Children's Oncology Group's 2013 blueprint for research: Soft tissue sarcomas. Pediatric blood \& cancer 60, 1001-1008.

Hennessy, B.T., Smith, D.L., Ram, P.T., Lu, Y., Mills, G.B., 2005. Exploiting the PI3K/AKT pathway for cancer drug discovery. Nature reviews. Drug discovery 4, 988-1004.

Hirai, H., Sootome, H., Nakatsuru, Y., Miyama, K., Taguchi, S., Tsujioka, K., Ueno, Y., Hatch, H., Majumder, P.K., Pan, B.S., Kotani, H., 2010. MK-2206, an allosteric Akt inhibitor, enhances antitumor efficacy by standard chemotherapeutic agents or molecular targeted drugs in vitro and in vivo. Molecular cancer therapeutics 9, 1956-1967.

Houghton, P.J., Morton, C.L., Kolb, E.A., Gorlick, R., Lock, R., Carol, H., Reynolds, C.P., Maris, J.M., Keir, S.T., Billups, C.A., Smith, M.A., 2008. Initial testing (stage 1) of the 
mTOR inhibitor rapamycin by the pediatric preclinical testing program. Pediatric blood \& cancer 50, 799-805.

Hsu, P.P., Sabatini, D.M., 2008. Cancer cell metabolism: Warburg and beyond. Cell 134, 703707.

Jalili, A., Mertz, K.D., Romanov, J., Wagner, C., Kalthoff, F., Stuetz, A., Pathria, G., Gschaider, M., Stingl, G., Wagner, S.N., 2013. NVP-LDE225, a potent and selective SMOOTHENED antagonist reduces melanoma growth in vitro and in vivo. PloS one 8, e69064.

Ji, Z., Mei, F.C., Xie, J., Cheng, X., 2007. Oncogenic KRAS activates hedgehog signaling pathway in pancreatic cancer cells. The Journal of biological chemistry 282, 1404814055.

Jiang, J., Hui, C.C., 2008. Hedgehog signaling in development and cancer. Developmental cell $15,801-812$.

Jimenez-Sanchez, M., Menzies, F.M., Chang, Y.Y., Simecek, N., Neufeld, T.P., Rubinsztein, D.C., 2012. The Hedgehog signalling pathway regulates autophagy. Nature communications 3, 1200.

Johnson, R.L., Rothman, A.L., Xie, J., Goodrich, L.V., Bare, J.W., Bonifas, J.M., Quinn, A.G., Myers, R.M., Cox, D.R., Epstein, E.H., Jr., Scott, M.P., 1996. Human homolog of patched, a candidate gene for the basal cell nevus syndrome. Science 272, 1668-1671.

Kang, M.H., Smith, M.A., Morton, C.L., Keshelava, N., Houghton, P.J., Reynolds, C.P., 2011. National Cancer Institute pediatric preclinical testing program: model description for in vitro cytotoxicity testing. Pediatric blood \& cancer 56, 239-249.

Kappler, R., Calzada-Wack, J., Schnitzbauer, U., Koleva, M., Herwig, A., Piontek, G., Graedler, F., Adamski, J., Heinzmann, U., Schlegel, J., Hemmerlein, B., Quintanilla-Martinez, L., Hahn, H., 2003. Molecular characterization of Patched-associated rhabdomyosarcoma. The Journal of pathology 200, 348-356.

Karhadkar, S.S., Steven Bova, G., Abdallah, N., Dhara, S., Gardner, D., Maitra, A., Isaacs, J.T., Berman, D.M., Beachy, P.A., 2004. Hedgehog signalling in prostate regeneration, neoplasia and metastasis. Nature.

Kaylani, S.Z., Xu, J., Srivastava, R.K., Kopelovich, L., Pressey, J.G., Athar, M., 2013. Rapamycin targeting mTOR and hedgehog signaling pathways blocks human rhabdomyosarcoma growth in xenograft murine model. Biochem Biophys Res Commun 435, 557-561.

Kenney, A.M., Cole, M.D., Rowitch, D.H., 2003. Nmyc upregulation by sonic hedgehog signaling promotes proliferation in developing cerebellar granule neuron precursors. Development 130, 15-28.

Kenney, A.M., Rowitch, D.H., 2000. Sonic hedgehog promotes G(1) cyclin expression and sustained cell cycle progression in mammalian neuronal precursors. Molecular and cellular biology 20, 9055-9067.

Kimura, H., Ng, J.M., Curran, T., 2008. Transient inhibition of the Hedgehog pathway in young mice causes permanent defects in bone structure. Cancer cell 13, 249-260.

Kool, M., Jones, D.T., Jager, N., Northcott, P.A., Pugh, T.J., Hovestadt, V., Piro, R.M., Esparza, L.A., Markant, S.L., Remke, M., Milde, T., Bourdeaut, F., Ryzhova, M., Sturm, D., Pfaff, E., Stark, S., Hutter, S., Seker-Cin, H., Johann, P., Bender, S., Schmidt, C., Rausch, T., Shih, D., Reimand, J., Sieber, L., Wittmann, A., Linke, L., Witt, H., Weber, U.D., Zapatka, M., Konig, R., Beroukhim, R., Bergthold, G., van Sluis, P., Volckmann, R., Koster, J., Versteeg, R., Schmidt, S., Wolf, S., Lawerenz, C., Bartholomae, C.C., von Kalle, C., Unterberg, A., Herold-Mende, C., Hofer, S., Kulozik, A.E., von Deimling, A., Scheurlen, W., Felsberg, J., Reifenberger, G., Hasselblatt, M., Crawford, J.R., Grant, G.A., Jabado, N., Perry, A., Cowdrey, C., Croul, S., Zadeh, G., Korbel, J.O., Doz, F., 
Delattre, O., Bader, G.D., McCabe, M.G., Collins, V.P., Kieran, M.W., Cho, Y.J., Pomeroy, S.L., Witt, O., Brors, B., Taylor, M.D., Schuller, U., Korshunov, A., Eils, R., Wechsler-Reya, R.J., Lichter, P., Pfister, S.M., 2014. Genome sequencing of SHH medulloblastoma predicts genotype-related response to smoothened inhibition. Cancer cell 25, 393-405.

Kramer, S., Meadows, A.T., Jarrett, P., Evans, A.E., 1983. Incidence of childhood cancer: experience of a decade in a population-based registry. Journal of the National Cancer Institute 70, 49-55.

Kubo, M., Nakamura, M., Tasaki, A., Yamanaka, N., Nakashima, H., Nomura, M., Kuroki, S., Katano, M., 2004. Hedgehog signaling pathway is a new therapeutic target for patients with breast cancer. Cancer research 64, 6071-6074.

Lam, C.W., Xie, J., To, K.F., Ng, H.K., Lee, K.C., Yuen, N.W., Lim, P.L., Chan, L.Y., Tong, S.F., McCormick, F., 1999. A frequent activated smoothened mutation in sporadic basal cell carcinomas. Oncogene 18, 833-836.

Lauth, M., Bergstrom, A., Toftgard, R., 2007. Phorbol esters inhibit the Hedgehog signalling pathway downstream of Suppressor of Fused, but upstream of Gli. Oncogene 26, 51635168.

Law, B.K., 2005. Rapamycin: an anti-cancer immunosuppressant? Critical reviews in oncology/hematology 56, 47-60.

Le Good, J.A., Ziegler, W.H., Parekh, D.B., Alessi, D.R., Cohen, P., Parker, P.J., 1998. Protein kinase $\mathrm{C}$ isotypes controlled by phosphoinositide 3-kinase through the protein kinase PDK1. Science 281, 2042-2045.

Lim, H.J., Crowe, P., Yang, J.L., 2015. Current clinical regulation of PI3K/PTEN/Akt/mTOR signalling in treatment of human cancer. Journal of cancer research and clinical oncology 141, 671-689.

Lindemann, R.K., 2008. Stroma-initiated hedgehog signaling takes center stage in B-cell lymphoma. Cancer research 68, 961-964.

Lipinski, R.J., Hutson, P.R., Hannam, P.W., Nydza, R.J., Washington, I.M., Moore, R.W., Girdaukas, G.G., Peterson, R.E., Bushman, W., 2008. Dose- and route-dependent teratogenicity, toxicity, and pharmacokinetic profiles of the hedgehog signaling antagonist cyclopamine in the mouse. Toxicological sciences : an official journal of the Society of Toxicology 104, 189-197.

LoRusso, P.M., Rudin, C.M., Reddy, J.C., Tibes, R., Weiss, G.J., Borad, M.J., Hann, C.L., Brahmer, J.R., Chang, I., Darbonne, W.C., Graham, R.A., Zerivitz, K.L., Low, J.A., Von Hoff, D.D., 2011. Phase I trial of hedgehog pathway inhibitor vismodegib (GDC-0449) in patients with refractory, locally advanced or metastatic solid tumors. Clinical cancer research : an official journal of the American Association for Cancer Research 17, 25022511.

Makawita, S., Ho, M., Durbin, A.D., Thorner, P.S., Malkin, D., Somers, G.R., 2009. Expression of insulin-like growth factor pathway proteins in rhabdomyosarcoma: IGF-2 expression is associated with translocation-negative tumors. Pediatric and developmental pathology : the official journal of the Society for Pediatric Pathology and the Paediatric Pathology Society $12,127-135$.

Markman, B., Dienstmann, R., Tabernero, J., 2010. Targeting the PI3K/Akt/mTOR pathway-beyond rapalogs. Oncotarget 1, 530-543.

Martins, A.S., Olmos, D., Missiaglia, E., Shipley, J., 2011. Targeting the insulin-like growth factor pathway in rhabdomyosarcomas: rationale and future perspectives. Sarcoma 2011, 209736.

McMahon, A.P., Ingham, P.W., Tabin, C.J., 2003. Developmental roles and clinical significance of hedgehog signaling. Curr Top Dev Biol 53, 1-114. 


\section{REFERENCES}

Merlino, G., Helman, L.J., 1999. Rhabdomyosarcoma-working out the pathways. Oncogene 18, 5340-5348.

Minniti, C.P., Tsokos, M., Newton, W.A., Jr., Helman, L.J., 1994. Specific expression of insulinlike growth factor-II in rhabdomyosarcoma tumor cells. American journal of clinical pathology 101, 198-203.

Mizushima, N., Yoshimori, T., Levine, B., 2010. Methods in mammalian autophagy research. Cell 140, 313-326.

Morton, J.P., Mongeau, M.E., Klimstra, D.S., Morris, J.P., Lee, Y.C., Kawaguchi, Y., Wright, C.V., Hebrok, M., Lewis, B.C., 2007. Sonic hedgehog acts at multiple stages during pancreatic tumorigenesis. Proceedings of the National Academy of Sciences of the United States of America 104, 5103-5108.

Mukherjee, S., Frolova, N., Sadlonova, A., Novak, Z., Steg, A., Page, G.P., Welch, D.R., LoboRuppert, S.M., Ruppert, J.M., Johnson, M.R., Frost, A.R., 2006. Hedgehog signaling and response to cyclopamine differ in epithelial and stromal cells in benign breast and breast cancer. Cancer biology \& therapy 5, 674-683.

Ng, J.M., Curran, T., 2011. The Hedgehog's tale: developing strategies for targeting cancer. Nature reviews. Cancer 11, 493-501.

Nikoletopoulou, V., Markaki, M., Palikaras, K., Tavernarakis, N., 2013. Crosstalk between apoptosis, necrosis and autophagy. Biochimica et biophysica acta 1833, 3448-3459.

Nilsson, M., Unden, A.B., Krause, D., Malmqwist, U., Raza, K., Zaphiropoulos, P.G., Toftgard, R., 2000. Induction of basal cell carcinomas and trichoepitheliomas in mice overexpressing GLI-1 [In Process Citation]. Proceedings of the National Academy of Sciences of the United States of America 97, 3438-3443.

Nolan-Stevaux, O., Lau, J., Truitt, M.L., Chu, G.C., Hebrok, M., Fernandez-Zapico, M.E., Hanahan, D., 2009. GLI1 is regulated through Smoothened-independent mechanisms in neoplastic pancreatic ducts and mediates PDAC cell survival and transformation. Genes \& development 23, 24-36.

Nusslein-Volhard, C., Wieschaus, E., 1980. Mutations affecting segment number and polarity in Drosophila. Nature 287, 795-801.

O'Reilly, K.E., Rojo, F., She, Q.B., Solit, D., Mills, G.B., Smith, D., Lane, H., Hofmann, F., Hicklin, D.J., Ludwig, D.L., Baselga, J., Rosen, N., 2006. mTOR inhibition induces upstream receptor tyrosine kinase signaling and activates Akt. Cancer research 66, 15001508.

Ognjanovic, S., Linabery, A.M., Charbonneau, B., Ross, J.A., 2009. Trends in childhood rhabdomyosarcoma incidence and survival in the United States, 1975-2005. Cancer 115, 4218-4226.

Ohtsu, A., Ajani, J.A., Bai, Y.X., Bang, Y.J., Chung, H.C., Pan, H.M., Sahmoud, T., Shen, L., Yeh, K.H., Chin, K., Muro, K., Kim, Y.H., Ferry, D., Tebbutt, N.C., Al-Batran, S.E., Smith, H., Costantini, C., Rizvi, S., Lebwohl, D., Van Cutsem, E., 2013. Everolimus for previously treated advanced gastric cancer: results of the randomized, double-blind, phase III GRANITE-1 study. Journal of clinical oncology : official journal of the American Society of Clinical Oncology 31, 3935-3943.

Oro, A.E., Higgins, K.M., Hu, Z., Bonifas, J.M., Epstein, E.H., Jr., Scott, M.P., 1997. Basal cell carcinomas in mice overexpressing sonic hedgehog. Science 276, 817-821.

Osborne, D.R., Yan, S., Stuckey, A., Pryer, L., Richey, T., Wall, J.S., 2012. Characterization of X-ray Dose in Murine Animals Using microCT, a New Low-Dose Detector and nanoDot Dosimeters. PloS one 7, e49936.

Paget, C., Duret, H., Ngiow, S.F., Kansara, M., Thomas, D.M., Smyth, M.J., 2012. Studying the role of the immune system on the antitumor activity of a Hedgehog inhibitor against murine osteosarcoma. Oncoimmunology 1, 1313-1322. 
Pan, S., Wu, X., Jiang, J., Gao, W., Wan, Y., Cheng, D., Han, D., Liu, J., Englund, N.P., Wang, Y., Peukert, S., Miller-Moslin, K., Yuan, J., Guo, R., Matsumoto, M., Vattay, A., Jiang, Y., Tsao, J., Sun, F., Pferdekamper, A.C., Dodd, S., Tuntland, T., Maniara, W., Kelleher, J.F., 3rd, Yao, Y.M., Warmuth, M., Williams, J., Dorsch, M., 2010. Discovery of NVPLDE225, a Potent and Selective Smoothened Antagonist. ACS medicinal chemistry letters 1, 130-134.

Petricoin, E.F., 3rd, Espina, V., Araujo, R.P., Midura, B., Yeung, C., Wan, X., Eichler, G.S., Johann, D.J., Jr., Qualman, S., Tsokos, M., Krishnan, K., Helman, L.J., Liotta, L.A., 2007. Phosphoprotein pathway mapping: Akt/mammalian target of rapamycin activation is negatively associated with childhood rhabdomyosarcoma survival. Cancer research 67, 3431-3440.

Petrova, R., Joyner, A.L., 2014. Roles for Hedgehog signaling in adult organ homeostasis and repair. Development 141, 3445-3457.

Pola, R., Ling, L.E., Silver, M., Corbley, M.J., Kearney, M., Blake Pepinsky, R., Shapiro, R., Taylor, F.R., Baker, D.P., Asahara, T., Isner, J.M., 2001. The morphogen Sonic hedgehog is an indirect angiogenic agent upregulating two families of angiogenic growth factors. Nature medicine 7, 706-711.

Polizio, A.H., Chinchilla, P., Chen, X., Kim, S., Manning, D.R., Riobo, N.A., 2011. Heterotrimeric Gi proteins link Hedgehog signaling to activation of Rho small GTPases to promote fibroblast migration. The Journal of biological chemistry 286, 19589-19596.

Pollak, M., 2008. Insulin and insulin-like growth factor signalling in neoplasia. Nature reviews. Cancer 8, 915-928.

Pressey, J.G., Anderson, J.R., Crossman, D.K., Lynch, J.C., Barr, F.G., 2011. Hedgehog pathway activity in pediatric embryonal rhabdomyosarcoma and undifferentiated sarcoma: A report from the Children's Oncology Group. Pediatric blood \& cancer.

Preuss, E., Hugle, M., Reimann, R., Schlecht, M., Fulda, S., 2013. Pan-mammalian target of rapamycin (mTOR) inhibitor AZD8055 primes rhabdomyosarcoma cells for ABT-737induced apoptosis by down-regulating Mcl-1 protein. The Journal of biological chemistry 288, 35287-35296.

Qualtrough, D., Buda, A., Gaffield, W., Williams, A.C., Paraskeva, C., 2004. Hedgehog signalling in colorectal tumour cells: induction of apoptosis with cyclopamine treatment. International journal of cancer. Journal international du cancer 110, 831-837.

Ragazzini, P., Gamberi, G., Pazzaglia, L., Serra, M., Magagnoli, G., Ponticelli, F., Ferrari, C., Ghinelli, C., Alberghini, M., Bertoni, F., Picci, P., Benassi, M.S., 2004. Amplification of CDK4, MDM2, SAS and GLI genes in leiomyosarcoma, alveolar and embryonal rhabdomyosarcoma. Histol Histopathol 19, 401-411.

Ramaswamy, B., Lu, Y., Teng, K.Y., Nuovo, G., Li, X., Shapiro, C.L., Majumder, S., 2012. Hedgehog signaling is a novel therapeutic target in tamoxifen-resistant breast cancer aberrantly activated by PI3K/AKT pathway. Cancer research 72, 5048-5059.

Rao, G., Pedone, C.A., Valle, L.D., Reiss, K., Holland, E.C., Fults, D.W., 2004. Sonic hedgehog and insulin-like growth factor signaling synergize to induce medulloblastoma formation from nestin-expressing neural progenitors in mice. Oncogene 23, 6156-6162.

Ray-Coquard, I., Favier, L., Weber, B., Roemer-Becuwe, C., Bougnoux, P., Fabbro, M., Floquet, A., Joly, F., Plantade, A., Paraiso, D., Pujade-Lauraine, E., 2013. Everolimus as secondor third-line treatment of advanced endometrial cancer: ENDORAD, a phase II trial of GINECO. British journal of cancer 108, 1771-1777.

Raynaud, F.I., Eccles, S., Clarke, P.A., Hayes, A., Nutley, B., Alix, S., Henley, A., Di-Stefano, F., Ahmad, Z., Guillard, S., Bjerke, L.M., Kelland, L., Valenti, M., Patterson, L., Gowan, S., de Haven Brandon, A., Hayakawa, M., Kaizawa, H., Koizumi, T., Ohishi, T., Patel, S., Saghir, N., Parker, P., Waterfield, M., Workman, P., 2007. Pharmacologic 
characterization of a potent inhibitor of class I phosphatidylinositide 3-kinases. Cancer research 67, 5840-5850.

Raynaud, F.I., Eccles, S.A., Patel, S., Alix, S., Box, G., Chuckowree, I., Folkes, A., Gowan, S., De Haven Brandon, A., Di Stefano, F., Hayes, A., Henley, A.T., Lensun, L., PerglWilson, G., Robson, A., Saghir, N., Zhyvoloup, A., McDonald, E., Sheldrake, P., Shuttleworth, S., Valenti, M., Wan, N.C., Clarke, P.A., Workman, P., 2009. Biological properties of potent inhibitors of class I phosphatidylinositide 3-kinases: from PI-103 through PI-540, PI-620 to the oral agent GDC-0941. Molecular cancer therapeutics 8, 1725-1738.

Regl, G., Kasper, M., Schnidar, H., Eichberger, T., Neill, G.W., Philpott, M.P., Esterbauer, H., Hauser-Kronberger, C., Frischauf, A.M., Aberger, F., 2004. Activation of the BCL2 promoter in response to Hedgehog/GLI signal transduction is predominantly mediated by GLI2. Cancer research 64, 7724-7731.

Renshaw, J., Taylor, K.R., Bishop, R., Valenti, M., De Haven Brandon, A., Gowan, S., Eccles, S.A., Ruddle, R.R., Johnson, L.D., Raynaud, F.I., Selfe, J.L., Thway, K., Pietsch, T., Pearson, A.D., Shipley, J., 2013. Dual blockade of the PI3K/AKT/mTOR (AZD8055) and RAS/MEK/ERK (AZD6244) pathways synergistically inhibits rhabdomyosarcoma cell growth in vitro and in vivo. Clinical cancer research : an official journal of the American Association for Cancer Research 19, 5940-5951.

Ridzewski, R., Rettberg, D., Dittmann, K., Cuvelier, N., Fulda, S., Hahn, H., 2015. Hedgehog Inhibitors in Rhabdomyosarcoma: A Comparison of Four Compounds and Responsiveness of Four Cell Lines. Frontiers in oncology 5, 130.

Riobo, N.A., Lu, K., Ai, X., Haines, G.M., Emerson, C.P., Jr., 2006. Phosphoinositide 3-kinase and Akt are essential for Sonic Hedgehog signaling. Proceedings of the National Academy of Sciences of the United States of America 103, 4505-4510.

Robarge, K.D., Brunton, S.A., Castanedo, G.M., Cui, Y., Dina, M.S., Goldsmith, R., Gould, S.E., Guichert, O., Gunzner, J.L., Halladay, J., Jia, W., Khojasteh, C., Koehler, M.F., Kotkow, K., La, H., Lalonde, R.L., Lau, K., Lee, L., Marshall, D., Marsters, J.C., Jr., Murray, L.J., Qian, C., Rubin, L.L., Salphati, L., Stanley, M.S., Stibbard, J.H., Sutherlin, D.P., Ubhayaker, S., Wang, S., Wong, S., Xie, M., 2009. GDC-0449-a potent inhibitor of the hedgehog pathway. Bioorganic \& medicinal chemistry letters 19, 5576-5581.

Robbins, D.J., Fei, D.L., Riobo, N.A., 2012. The Hedgehog signal transduction network. Science signaling 5 , re6.

Roberts, W.M., Douglass, E.C., Peiper, S.C., Houghton, P.J., Look, A.T., 1989. Amplification of the gli gene in childhood sarcomas. Cancer research 49, 5407-5413.

Rohatgi, R., Milenkovic, L., Corcoran, R.B., Scott, M.P., 2009. Hedgehog signal transduction by Smoothened: pharmacologic evidence for a 2-step activation process. Proceedings of the National Academy of Sciences of the United States of America 106, 3196-3201.

Rohatgi, R., Scott, M.P., 2007. Patching the gaps in Hedgehog signalling. Nat Cell Biol 9, 10051009.

Romer, J.T., Kimura, H., Magdaleno, S., Sasai, K., Fuller, C., Baines, H., Connelly, M., Stewart, C.F., Gould, S., Rubin, L.L., Curran, T., 2004. Suppression of the Shh pathway using a small molecule inhibitor eliminates medulloblastoma in Ptc1(+/-)p53(-/-) mice. Cancer cell 6, 229-240.

Ruat, M., Hoch, L., Faure, H., Rognan, D., 2014. Targeting of Smoothened for therapeutic gain. Trends in pharmacological sciences 35, 237-246.

Rudin, C.M., 2012. Vismodegib. Clinical cancer research : an official journal of the American Association for Cancer Research 18, 3218-3222.

Rudin, C.M., Hann, C.L., Laterra, J., Yauch, R.L., Callahan, C.A., Fu, L., Holcomb, T., Stinson, J., Gould, S.E., Coleman, B., LoRusso, P.M., Von Hoff, D.D., de Sauvage, F.J., Low, 
J.A., 2009. Treatment of medulloblastoma with hedgehog pathway inhibitor GDC-0449. The New England journal of medicine 361, 1173-1178.

Ruiz i Altaba, A., Mas, C., Stecca, B., 2007. The Gli code: an information nexus regulating cell fate, stemness and cancer. Trends in cell biology 17, 438-447.

Ryan, K.E., Chiang, C., 2012. Hedgehog secretion and signal transduction in vertebrates. The Journal of biological chemistry 287, 17905-17913.

Sanchez, P., Hernandez, A.M., Stecca, B., Kahler, A.J., DeGueme, A.M., Barrett, A., Beyna, M., Datta, M.W., Datta, S., Ruiz i Altaba, A., 2004. Inhibition of prostate cancer proliferation by interference with SONIC HEDGEHOG-GLI1 signaling. Proceedings of the National Academy of Sciences of the United States of America 101, 12561-12566.

Sanchez, P., Ruiz i Altaba, A., 2005. In vivo inhibition of endogenous brain tumors through systemic interference of Hedgehog signaling in mice. Mech Dev 122, 223-230.

Sarbassov, D.D., Ali, S.M., Sengupta, S., Sheen, J.H., Hsu, P.P., Bagley, A.F., Markhard, A.L., Sabatini, D.M., 2006. Prolonged rapamycin treatment inhibits mTORC2 assembly and Akt/PKB. Molecular cell 22, 159-168.

Sarbassov, D.D., Guertin, D.A., Ali, S.M., Sabatini, D.M., 2005. Phosphorylation and regulation of Akt/PKB by the rictor-mTOR complex. Science 307, 1098-1101.

Scales, S.J., de Sauvage, F.J., 2009. Mechanisms of Hedgehog pathway activation in cancer and implications for therapy. Trends in pharmacological sciences.

Schindelin, J., Arganda-Carreras, I., Frise, E., Kaynig, V., Longair, M., Pietzsch, T., Preibisch, S., Rueden, C., Saalfeld, S., Schmid, B., Tinevez, J.Y., White, D.J., Hartenstein, V., Eliceiri, K., Tomancak, P., Cardona, A., 2012. Fiji: an open-source platform for biological-image analysis. Nat Methods 9, 676-682.

Sekulic, A., Migden, M.R., Oro, A.E., Dirix, L., Lewis, K.D., Hainsworth, J.D., Solomon, J.A., Yoo, S., Arron, S.T., Friedlander, P.A., Marmur, E., Rudin, C.M., Chang, A.L., Low, J.A., Mackey, H.M., Yauch, R.L., Graham, R.A., Reddy, J.C., Hauschild, A., 2012. Efficacy and safety of vismodegib in advanced basal-cell carcinoma. The New England journal of medicine 366, 2171-2179.

Stecca, B., Mas, C., Clement, V., Zbinden, M., Correa, R., Piguet, V., Beermann, F., Ruiz, I.A.A., 2007. Melanomas require HEDGEHOG-GLI signaling regulated by interactions between GLI1 and the RAS-MEK/AKT pathways. Proceedings of the National Academy of Sciences of the United States of America 104, 5895-5900.

Stecca, B., Ruiz, I.A.A., 2010. Context-dependent regulation of the GLI code in cancer by HEDGEHOG and non-HEDGEHOG signals. J Mol Cell Biol 2, 84-95.

Struffert, T., Doelken, M., Adamek, E., Schwarz, M., Engelhorn, T., Kloska, S., Ott, S., Doerfler, A., 2010. Flat-detector computed tomography with intravenous contrast material application in experimental aneurysms: comparison with multislice CT and conventional angiography. Acta Radiol 51, 431-437.

Taipale, J., Chen, J.K., Cooper, M.K., Wang, B., Mann, R.K., Milenkovic, L., Scott, M.P., Beachy, P.A., 2000. Effects of oncogenic mutations in Smoothened and Patched can be reversed by cyclopamine. Nature 406, 1005-1009.

Taipale, J., Cooper, M.K., Maiti, T., Beachy, P.A., 2002. Patched acts catalytically to suppress the activity of Smoothened. Nature 418, 892-897.

Tamburini, J., Chapuis, N., Bardet, V., Park, S., Sujobert, P., Willems, L., Ifrah, N., Dreyfus, F., Mayeux, P., Lacombe, C., Bouscary, D., 2008. Mammalian target of rapamycin (mTOR) inhibition activates phosphatidylinositol 3-kinase/Akt by up-regulating insulin-like growth factor-1 receptor signaling in acute myeloid leukemia: rationale for therapeutic inhibition of both pathways. Blood 111, 379-382.

Tang, J.Y., Mackay-Wiggan, J.M., Aszterbaum, M., Yauch, R.L., Lindgren, J., Chang, K., Coppola, C., Chanana, A.M., Marji, J., Bickers, D.R., Epstein, E.H., Jr., 2012. Inhibiting 
the hedgehog pathway in patients with the basal-cell nevus syndrome. The New England journal of medicine 366, 2180-2188.

Tao, H., Jin, Q., Koo, D.I., Liao, X., Englund, N.P., Wang, Y., Ramamurthy, A., Schultz, P.G., Dorsch, M., Kelleher, J., Wu, X., 2011. Small molecule antagonists in distinct binding modes inhibit drug-resistant mutant of smoothened. Chemistry \& biology 18, 432-437.

Taylor, M.D., Liu, L., Raffel, C., Hui, C.C., Mainprize, T.G., Zhang, X., Agatep, R., Chiappa, S., Gao, L., Lowrance, A., Hao, A., Goldstein, A.M., Stavrou, T., Scherer, S.W., Dura, W.T., Wainwright, B., Squire, J.A., Rutka, J.T., Hogg, D., 2002. Mutations in SUFU predispose to medulloblastoma. Nat Genet 31, 306-310.

Teperino, R., Aberger, F., Esterbauer, H., Riobo, N., Pospisilik, J.A., 2014. Canonical and noncanonical Hedgehog signalling and the control of metabolism. Seminars in cell \& developmental biology 33, 81-92.

Teperino, R., Amann, S., Bayer, M., McGee, S.L., Loipetzberger, A., Connor, T., Jaeger, C., Kammerer, B., Winter, L., Wiche, G., Dalgaard, K., Selvaraj, M., Gaster, M., LeeYoung, R.S., Febbraio, M.A., Knauf, C., Cani, P.D., Aberger, F., Penninger, J.M., Pospisilik, J.A., Esterbauer, H., 2012. Hedgehog partial agonism drives Warburg-like metabolism in muscle and brown fat. Cell 151, 414-426.

Thayer, S.P., di Magliano, M.P., Heiser, P.W., Nielsen, C.M., Roberts, D.J., Lauwers, G.Y., Qi, Y.P., Gysin, S., Fernandez-del Castillo, C., Yajnik, V., Antoniu, B., McMahon, M., Warshaw, A.L., Hebrok, M., 2003. Hedgehog is an early and late mediator of pancreatic cancer tumorigenesis. Nature 425, 851-856.

Thibert, C., Teillet, M.A., Lapointe, F., Mazelin, L., Le Douarin, N.M., Mehlen, P., 2003. Inhibition of neuroepithelial patched-induced apoptosis by sonic hedgehog. Science 301, 843-846.

Tostar, U., Malm, C.J., Meis-Kindblom, J.M., Kindblom, L.G., Toftgard, R., Unden, A.B., 2006. Deregulation of the hedgehog signalling pathway: a possible role for the PTCH and SUFU genes in human rhabdomyoma and rhabdomyosarcoma development. The Journal of pathology 208, 17-25.

Tostar, U., Toftgard, R., Zaphiropoulos, P.G., Shimokawa, T., 2010. Reduction of human embryonal rhabdomyosarcoma tumor growth by inhibition of the hedgehog signaling pathway. Genes Cancer 1, 941-951.

Vemulapalli, S., Mita, A., Alvarado, Y., Sankhala, K., Mita, M., 2011. The emerging role of mammalian target of rapamycin inhibitors in the treatment of sarcomas. Targeted oncology 6, 29-39.

Vivanco, I., Sawyers, C.L., 2002. The phosphatidylinositol 3-Kinase AKT pathway in human cancer. Nature reviews. Cancer 2, 489-501.

Wan, X., Harkavy, B., Shen, N., Grohar, P., Helman, L.J., 2007. Rapamycin induces feedback activation of Akt signaling through an IGF-1R-dependent mechanism. Oncogene 26, 1932-1940.

Wan, X., Helman, L.J., 2007. The biology behind mTOR inhibition in sarcoma. The oncologist 12, 1007-1018.

Wang, C., Wu, H., Evron, T., Vardy, E., Han, G.W., Huang, X.P., Hufeisen, S.J., Mangano, T.J., Urban, D.J., Katritch, V., Cherezov, V., Caron, M.G., Roth, B.L., Stevens, R.C., 2014. Structural basis for Smoothened receptor modulation and chemoresistance to anticancer drugs. Nature communications 5, 4355.

Wang, Y., Ding, Q., Yen, C.J., Xia, W., Izzo, J.G., Lang, J.Y., Li, C.W., Hsu, J.L., Miller, S.A., Wang, X., Lee, D.F., Hsu, J.M., Huo, L., Labaff, A.M., Liu, D., Huang, T.H., Lai, C.C., Tsai, F.J., Chang, W.C., Chen, C.H., Wu, T.T., Buttar, N.S., Wang, K.K., Wu, Y., Wang, H., Ajani, J., Hung, M.C., 2012. The crosstalk of mTOR/S6K1 and Hedgehog pathways. Cancer cell 21, 374-387. 
Wang, Y., Han, C., Lu, L., Magliato, S., Wu, T., 2013. Hedgehog signaling pathway regulates autophagy in human hepatocellular carcinoma cells. Hepatology 58, 995-1010.

Wang, Y., Zhou, Z., Walsh, C.T., McMahon, A.P., 2009. Selective translocation of intracellular Smoothened to the primary cilium in response to Hedgehog pathway modulation. Proceedings of the National Academy of Sciences of the United States of America 106, 2623-2628.

Wei, L., Xu, Z., 2011. Cross-signaling among phosphinositide-3 kinase, mitogen-activated protein kinase and sonic hedgehog pathways exists in esophageal cancer. International journal of cancer. Journal international du cancer 129, 275-284.

White, E., DiPaola, R.S., 2009. The double-edged sword of autophagy modulation in cancer. Clinical cancer research : an official journal of the American Association for Cancer Research 15, 5308-5316.

Wicking, C., Smyth, I., Bale, A., 1999. The hedgehog signalling pathway in tumorigenesis and development. Oncogene 18, 7844-7851.

Wilson, C.W., Chen, M.H., Chuang, P.T., 2009. Smoothened adopts multiple active and inactive conformations capable of trafficking to the primary cilium. PloS one 4, e5182.

Wilson, E.M., Hsieh, M.M., Rotwein, P., 2003. Autocrine growth factor signaling by insulin-like growth factor-II mediates MyoD-stimulated myocyte maturation. The Journal of biological chemistry 278, 41109-41113.

Wilson, E.M., Rotwein, P., 2006. Control of MyoD function during initiation of muscle differentiation by an autocrine signaling pathway activated by insulin-like growth factorII. The Journal of biological chemistry 281, 29962-29971.

Wong, H., Chen, J.Z., Chou, B., Halladay, J.S., Kenny, J.R., La, H., Marsters, J.C., Jr., Plise, E., Rudewicz, P.J., Robarge, K., Shin, Y., Wong, S., Zhang, C., Khojasteh, S.C., 2009. Preclinical assessment of the absorption, distribution, metabolism and excretion of GDC0449 (2-chloro-N-(4-chloro-3-(pyridin-2-yl)phenyl)-4-(methylsulfonyl)benzamide), an orally bioavailable systemic Hedgehog signalling pathway inhibitor. Xenobiotica; the fate of foreign compounds in biological systems 39, 850-861.

Workman, P., Clarke, P.A., Raynaud, F.I., van Montfort, R.L., 2010. Drugging the PI3 kinome: from chemical tools to drugs in the clinic. Cancer research 70, 2146-2157.

Wu, V.M., Chen, S.C., Arkin, M.R., Reiter, J.F., 2012. Small molecule inhibitors of Smoothened ciliary localization and ciliogenesis. Proceedings of the National Academy of Sciences of the United States of America 109, 13644-13649.

Xu, Y., An, Y., Wang, X., Zha, W., Li, X., 2014. Inhibition of the Hedgehog pathway induces autophagy in pancreatic ductal adenocarcinoma cells. Oncol Rep 31, 707-712.

Yam, P.T., Langlois, S.D., Morin, S., Charron, F., 2009. Sonic hedgehog guides axons through a noncanonical, Src-family-kinase-dependent signaling pathway. Neuron 62, 349-362.

Yap, T.A., Yan, L., Patnaik, A., Fearen, I., Olmos, D., Papadopoulos, K., Baird, R.D., Delgado, L., Taylor, A., Lupinacci, L., Riisnaes, R., Pope, L.L., Heaton, S.P., Thomas, G., Garrett, M.D., Sullivan, D.M., de Bono, J.S., Tolcher, A.W., 2011. First-in-man clinical trial of the oral pan-AKT inhibitor MK-2206 in patients with advanced solid tumors. Journal of clinical oncology : official journal of the American Society of Clinical Oncology 29, 4688-4695.

Yauch, R.L., Dijkgraaf, G.J., Alicke, B., Januario, T., Ahn, C.P., Holcomb, T., Pujara, K., Stinson, J., Callahan, C.A., Tang, T., Bazan, J.F., Kan, Z., Seshagiri, S., Hann, C.L., Gould, S.E., Low, J.A., Rudin, C.M., de Sauvage, F.J., 2009. Smoothened mutation confers resistance to a Hedgehog pathway inhibitor in medulloblastoma. Science 326, $572-574$. 


\section{REFERENCES}

Yauch, R.L., Gould, S.E., Scales, S.J., Tang, T., Tian, H., Ahn, C.P., Marshall, D., Fu, L., Januario, T., Kallop, D., Nannini-Pepe, M., Kotkow, K., Marsters, J.C., Rubin, L.L., de Sauvage, F.J., 2008. A paracrine requirement for hedgehog signalling in cancer. Nature.

Yoo, Y.A., Kang, M.H., Lee, H.J., Kim, B.H., Park, J.K., Kim, H.K., Kim, J.S., Oh, S.C., 2011. Sonic hedgehog pathway promotes metastasis and lymphangiogenesis via activation of Akt, EMT, and MMP-9 pathway in gastric cancer. Cancer research 71, 7061-7070.

Zhan, S., Shapiro, D.N., Helman, L.J., 1994. Activation of an imprinted allele of the insulin-like growth factor II gene implicated in rhabdomyosarcoma. The Journal of clinical investigation 94, 445-448.

Zhang, L., Kashanchi, F., Zhan, Q., Zhan, S., Brady, J.N., Fornace, A.J., Seth, P., Helman, L.J., 1996. Regulation of insulin-like growth factor II P3 promotor by p53: a potential mechanism for tumorigenesis. Cancer research 56, 1367-1373.

Zhang, L., Zhan, Q., Zhan, S., Kashanchi, F., Fornace, A.J., Jr., Seth, P., Helman, L.J., 1998a. p53 regulates human insulin-like growth factor II gene expression through active P4 promoter in rhabdomyosarcoma cells. DNA Cell Biol 17, 125-131.

Zhang, L., Zhan, S., Navid, F., Li, Q., Choi, Y.H., Kim, M., Seth, P., Helman, L.J., 1998b. AP-2 may contribute to IGF-II overexpression in rhabdomyosarcoma. Oncogene 17, 12611270.

Zhao, Y.Y., Tian, Y., Zhang, J., Xu, F., Yang, Y.P., Huang, Y., Zhao, H.Y., Zhang, J.W., Xue, C., Lam, M.H., Yan, L., Hu, Z.H., Dinglin, X.X., Zhang, L., 2014. Effects of an oral allosteric AKT inhibitor (MK-2206) on human nasopharyngeal cancer in vitro and in vivo. Drug design, development and therapy 8, 1827-1837.

Zibat, A., Missiaglia, E., Rosenberger, A., Pritchard-Jones, K., Shipley, J., Hahn, H., Fulda, S., 2010. Activation of the hedgehog pathway confers a poor prognosis in embryonal and fusion gene-negative alveolar rhabdomyosarcoma. Oncogene, Sep 6. [Epub ahead of print]. 


\section{ABBREVIATIONS}

\begin{tabular}{|c|c|}
\hline ARMS & alveolar rhabdomyosarcoma \\
\hline BAD & Bcl-2-associated death promoter \\
\hline BAX & Bcl-2-associated X protein \\
\hline BCA & bicinchoninic acid \\
\hline $\mathrm{BCC}$ & basal cell carcinoma \\
\hline $\mathrm{Bcl} 2$ & B-cell lymphoma 2 \\
\hline bp & base pairs \\
\hline BrdU & 5-bromo-2-deoxyuridine \\
\hline BSA & bovine serum albumin \\
\hline cDNA & complementary deoxyribonucleic acid \\
\hline CRC & colorectal cancer \\
\hline $\mathrm{ddH}_{2} \mathrm{O}$ & double distilled water \\
\hline DMEM & Dulbecco's Modified Eagle Medium \\
\hline DMSO & dimethyl sulfoxide \\
\hline DNA & deoxyribonucleic acid \\
\hline dNTP & deoxyribonucleotide triphosphate \\
\hline DOX & doxorubicin \\
\hline DTT & dithiothreitol \\
\hline EAC & esophageal adenocarcinoma \\
\hline EDTA & ethylenediaminetetraacetic acid \\
\hline EGFR & epidermal growth factor receptor \\
\hline ERMS & embryonal rhabdomyosarcoma \\
\hline $\mathrm{EtOH}$ & ethanol \\
\hline FCS & fetal calf serum \\
\hline FITC & fluorescein isothiocyanate \\
\hline FOXO & forkhead transcription factor \\
\hline gDNA & genomic deoxyribonucleic acid \\
\hline $\mathrm{G}_{\mathrm{i}}$ & G inhibitory (subunit of guanine nucleotide binding proteins) \\
\hline
\end{tabular}


GLI

GPCR

GSK3

GTPase

HE

$\mathrm{HH}$

HRP

HSC70

IGF1/2R

IGF2

IHC

IRS

$\mathrm{LOH}$

MB

MCT

MDM2

mRNA

mTOR

Myc

MYH1

neo

PBS

PBST

PCR

PDK1

PFA

PI

PI3K

PIP2

PIP3

PKA

PKC

PMSF

PS glioma-associated oncogene

G-protein-coupled-receptor

glycogen synthase kinase 3

guanosine triphosphate hydrolase

haematoxylin eosin

hedgehog

horseradish peroxidase

heat shock protein 70

insulin-like growth factor $1 / 2$ receptor

insulin-like growth factor 2

immunohistochemistry

insulin receptor substrate

loss of heterozygosity

medulloblastoma

methyl cellulose tween

murine double minute 2

messenger ribonucleic acid

mammalian target of rapamycin

myelocytomatosis oncogene

myosin heavy chain 1

neomycin resistance

phosphate buffered saline

phosphate buffered saline -tween

polymerase chain reaction

phosphoinositide-dependent kinase 1

paraformaldehyde

propidium iodide

phosphatidylinositol-3-kinase

phosphatidylinositol 4,5-bisphosphate

phosphatidylinositol 3,4,5-trisphosphate

protein kinase $\mathrm{A}$

protein kinase $\mathrm{C}$

phenylmethanesulfonylfluoride

phosphatidylserine 
PTCH

PTEN

p53

$\mathrm{P} / \mathrm{S}$

qRT-PCR

$\mathrm{RCC}$

RhoA

RMS

RT

RTK

S6

S6K

SAG

SEM

SDS

$\mathrm{SHH}$

SMO

SUFU

TBE

TBS

TBST

VCT

$\mathrm{v} / \mathrm{v}$

WST

$\mathrm{w} / \mathrm{v}$ patched

phosphatase and tensin homolog deleted on chromosome 10

transformation related protein 53

penicillin/streptomycin

quantitative real-time polymerase chain reaction

renal cell carcinoma

ras homolog family member A

rhabdomyosarcoma

room temperature

receptor tyrosine kinases

ribosomal S6 protein

S6 kinase

smoothened agonist

standard error of the mean

sodiumdodecylsulfate

sonic hedgehog

smoothened

suppressor of fused

tris-boric acid-EDTA

tris-buffered saline

tris-buffered saline-Triton X-100

volumetric computer tomography

volume/volume

water soluble tetrazolium salt 1

weight/volume 


\section{ACKNOWLEDGEMENT}

I would like to thank the following people which supported me throughout my doctoral study and contributed to this thesis, which would not have been possible without their help.

First, I would like to thank Prof. Dr. Heidi Hahn for the opportunity to research on this project, for your guidance and supervision and for the confidence you placed in me. I sincerely appreciate your continuous help, your richness of ideas and that you were always willing to listen to my questions throughout my work.

I would also like to thank the members of my thesis committee Prof. Dr. Matthias Dobbelstein and Prof. Dr. Dieter Kube for your valuable inputs and your numerous ideas during the thesis committee meetings.

I also would like to acknowledge the DFG graduate school 1034 for the opportunity to be a member of this program and for the financial support.

Special thanks go to Dr. Kai Dittmann for your expertise in flow cytometry, for all your support, advice and your encouragement. Thank you for the enjoyable conversations Kai.

Many thanks go to all my colleagues in the laboratory. In particular, I would like to thank Dr. Anja Uhmann for your competent knowledge, huge helpfulness and for the exciting dialogues on scientific issues and all nonscientific, funny conversations. Moreover, I especially would like to thank Dr. Diana Rettberg for your guidance and encouragement in the beginning of my project. Many thanks go to Natalie Hönig for always being reliable. Schnatti it was great to work with you. Similarly, my gratitude goes to Anke Frommhold and Ina Heß for technical support and particularly to Anke for cutting all my slides. Many thanks also go to all the other past and present lab members: Dr. Simone König, Dr. Penelope Pelczar, Dr. Frauke Nitzki, Dr. Marco Becker, Dr. Benedikt Linder, Julia Dräger, Nicole Cuvelier, Joanna Pyczek and Julia Heise. Furthermore I would like to thank Christian Dullin for your great help and support during the VCT measurements. Big thanks go also to the animal care takers, especially Susann Peter and Jennifer Flemming for the excellent animal care. 
Most importantly, I would like to thank my brother Thomas, my sister Madeleine, my best friend Julia as well as my dear Adam for your constant support, patience and encouragement throughout the years. Julia, you are the most wonderful friend one can imagine. Thanks for your immense help, your lovely laughter, your gorgeous humor, for the great times and unforgettable moments we shared! Without you, this thesis would not have been possible! Adam, thank you for your love and for always being by my side. I am enormously grateful and proud that I got to know you guys and that you are my friends as well as my family.

Thank you for everything! 